\title{
An Improved $W$ Boson Mass Measurement Using the Collider Detector at Fermilab
}

by

Yu Zeng

Department of Physics

Duke University

Date:

Approved:

\begin{tabular}{c}
\hline Ashutosh V. Kotwal, Chair \\
\hline Alfred T. Goshaw \\
\hline Mark C. Kruse \\
\hline Steffen A. Bass \\
\hline M. Ronen Plesser
\end{tabular}

A dissertation submitted in partial fulfillment of the requirements for the degree of Doctor of Philosophy in the Department of Physics in the Graduate School of Duke University 


\section{$\underline{\text { ABSTRACT }}$}

\section{An Improved $W$ Boson Mass Measurement Using the Collider Detector at Fermilab \\ by}

Yu Zeng

Department of Physics

Duke University

Date:

Approved:

\begin{tabular}{c}
\hline Ashutosh V. Kotwal, Chair \\
\hline Alfred T. Goshaw \\
\hline Mark C. Kruse \\
\hline Steffen A. Bass \\
\hline M. Ronen Plesser
\end{tabular}

An abstract of a dissertation submitted in partial fulfillment of the requirements for the degree of Doctor of

Philosophy in the Department of Physics

in the Graduate School of

Duke University 
Copyright (c) 2012 by Yu Zeng

All rights reserved except the rights granted by the Creative Commons Attribution-Noncommercial Licence 


\section{Abstract}

The mass of the $W$ boson is one of the most important parameters in the Standard Model. A precise measurement of the $W$ boson mass, together with a precise measurement of the top quark mass, can constrain the mass of the undiscovered Higgs boson within the Standard Model framework or give a hint for physics beyond the Standard Model.

This dissertation describes a measurement of the $W$ boson mass through its decay into a muon and a neutrino using $\approx 2.2 \mathrm{fb}^{-1}$ of $\sqrt{s}=1.96 \mathrm{TeV} p \bar{p}$ data taken with the CDF II detector at Fermilab. We measure the $W$ boson mass to be $\left(80.374 \pm 0.015_{\text {stat. }} \pm 0.016_{\text {syst. }}\right) \mathrm{GeV} / \mathrm{c}^{2}$. This result, when combined with the $W$ mass measurement in the electron channel, leads to the single most precise $m_{W}$ value and greatly constrains the possible mass range of the undiscovered Higgs boson. 
To Evan \& Wenting 


\section{Contents}

Abstract $\quad$ iv

List of Tables $\quad$ xi

List of Figures $\quad$ xiv

$\begin{array}{ll}\text { Acknowledgements } & \text { Xxv }\end{array}$

1 Introduction 1

1.1 The Standard Model . . . . . . . . . . . . . . . . . . . 1

1.1.1 The Higgs Mechanism for Boson Masses . . . . . . . . . . . . 4

1.1.2 Mass Bounds of the Higgs Boson . . . . . . . . . . . . . . 9

1.2 Motivation for $W$ Boson Mass Measurement . . . . . . . . . . . . . . 12

1.3 Past $W$ Boson Mass Measurements . . . . . . . . . . . . . . . . . . . 16

1.4 Thesis Structure . . . . . . . . . . . . . . . 16

2 The Accelerator at Fermilab $\quad 19$

2.1 The Fermilab Accelerator Chain . . . . . . . . . . . . . . . . . 19

2.1.1 The Proton Source . . . . . . . . . . . . . . . 19

2.1 .2 The Main Injector . . . . . . . . . . . . . . . . . 21

2.1.3 The Anti-Proton Source . . . . . . . . . . . . . 21

2.1.4 The Recycler ... . . . . . . . . . . . . . 22

2.1.5 The Tevatron ................... . . 22

2.2 Tevatron Luminosity . . . . . . . . . . . . . . . . 23 
$\begin{array}{llr}3 & \text { Detector } & 27\end{array}$

3.1 The Coordinate System . . . . . . . . . . . . . . . . . 27

3.2 Silicon Tracker: Layer $00+$ SVX II + ISL . . . . . . . . . . . . . . 29

3.3 Central Outer Tracker: COT . . . . . . . . . . . . . 30

3.4 Calorimeter Systems . . . . . . . . . . . . . . . . . 33

3.5 Muon Systems . . . . . . . . . . . . . . . . . . . 35

3.6 Cherenkov Luminosity Counter . . . . . . . . . . . . . . 38

3.7 Data Acquisition System . . . . . . . . . . . . . . . 38

4 Measurement Strategy $\quad 42$

4.1 Jacobian Edge . . . . . . . . . . . . . . . . . . . 45

4.2 Strategy of this Measurement . . . . . . . . . . . . . . 47

4.3 Template Likelihood Fits . . . . . . . . . . . . . . . . . . . 49

4.4 Blinding Technique . . . . . . . . . . . . . . . 52

5 Dataset and Event Selection $\quad 53$

$5.1 W \rightarrow \mu \nu$ and $Z \rightarrow \mu \mu$ Samples . . . . . . . . . . . . 54

$5.2 J / \psi \rightarrow \mu \mu$ and $\Upsilon \rightarrow \mu \mu$ Samples . . . . . . . . . . . . . 58

$5.2 .1 \quad J / \psi \rightarrow \mu \mu$ Data Sample . . . . . . . . . . . . . . . . 59

$5.2 .2 \Upsilon \rightarrow \mu \mu$ Data Sample . . . . . . . . . . . . . . . 60

$6 \quad W$ Boson Production and Decay Model $\quad 63$

6.1 Parton Distribution Functions (PDF) . . . . . . . . . . . 63

$6.2 W$ and $Z$ Boson $p_{T}$ Model . . . . . . . . . . . . . 66

$6.3 W$ Boson Decay Model . . . . . . . . . . . . . . . . . . . . . . . 69

6.4 Photon Radiation . . . . . . . . . . . . . . . . . . . . . 72

$\begin{array}{lll}7 & \text { Fast Detector Simulation } & 77\end{array}$

7.1 Ionization Energy Loss . . . . . . . . . . . . . . . . 78 
7.2 Multiple Coulomb Scattering . . . . . . . . . . . . . . . . . 79

7.3 COT simulation and reconstruction . . . . . . . . . . . . . . 79

7.4 Muon Identification Efficiency Modelling . . . . . . . . . . . . . . . . 82

7.5 Muon Acceptance Modelling . . . . . . . . . . . . . . . 83

8 Momentum Scale $\quad 85$

8.1 COT Alignment . . . . . . . . . . . . . . 87

8.2 COT Momentum Scale from $J / \psi$ Analysis . . . . . . . . . . . . . . . 90

8.2.1 $J / \psi$ Event Generation . . . . . . . . . . . . . . . . . 90

$8.2 .2 \quad J / \psi$ Analysis . . . . . . . . . . . . . . . . . . . 92

8.2.3 Systematic Uncertainties in $J / \psi$ Analysis . . . . . . . . . . . . 98

8.3 COT Momentum Scale from $\Upsilon$ Analysis . . . . . . . . . . . . 100

8.4 Combined Momentum Scale from $J / \psi$ and $\Upsilon$ Analyses . . . . . . . . 109

8.5 Comparison with $200 \mathrm{pb}^{-1}$ Momentum Scale . . . . . . . . . . . . 111

8.6 Cross-check of COT Momentum Scale using $Z \rightarrow \mu \mu$ Events . . . . . 113

$9 \begin{array}{ll}\text { Backgrounds } & 116\end{array}$

$9.1 Z \rightarrow \mu \mu$ and $W \rightarrow \tau \nu \ldots \ldots \ldots \ldots$

9.1.1 $Z \rightarrow \mu \mu$ systematic uncertainties . . . . . . . . . . 119

9.1.2 $W \rightarrow \tau \nu$ systematic uncertainty . . . . . . . . . . . . 120

9.2 Decay in Flight . . . . . . . . . . . . . . . . . . . . . . 121

9.3 QCD Jets . . . . . . . . . . . . . . . . . . . . 123

9.4 Cosmic Background . . . . . . . . . . . . . . . . . . . 127

9.5 Background Systematic Uncertainties on $m_{W} \ldots \ldots$. . . . . . 130

10 Hadronic Recoil Model 133

10.1 Calorimeter-based Recoil Measurement . . . . . . . . . . . . 134

10.2 Hadronic Recoil Response . . . . . . . . . . . . . . . 136 
10.3 Hadronic Recoil Resolution . . . . . . . . . . . . . . . . . . . . . 137

10.4 Recoil Model Cross-Checks . . . . . . . . . . . . . . . . . . . . . . 141

11 Result 146

11.1 Fitted $m_{W}$ Central Value and Statistical Error . . . . . . . . . . . . . 147

11.2 Transverse Mass $m_{T}$ Fit Result . . . . . . . . . . . . . . . . . . 148

11.3 Charged Lepton $p_{T}^{\mu}$ Fit Result . . . . . . . . . . . . . . . . 150

11.4 Missing Transverse Momentum $p_{T}^{\nu}$ Fit Result . . . . . . . . . . . . . 150

11.5 Cross-Checks . . . . . . . . . . . . . . . . 151

11.6 Correlations between Mass Fits . . . . . . . . . . . . . . . . . 154

11.6.1 Monte Carlo Study of Statistical Correlation Coefficient Errors 156

11.7 Combined Mass Result . . . . . . . . . . . . . . . 157

12 Summary and Future Pespective $\quad 164$

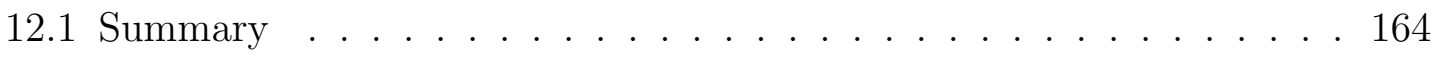

12.2 Future Perspective . . . . . . . . . . . . . . . 165

A PDF Uncertainties in $W \rightarrow e \nu$ channel $\quad 169$

$\begin{array}{ll}\text { B } Z / \gamma \text { interference } & 171\end{array}$

C Relationship between $\Delta p / p$ and $\left\langle 1 / p_{T}^{\mu}\right\rangle \quad 173$

D Individual $J / \psi$ Fits in $\left\langle 1 / p_{T}^{\mu}\right\rangle$ Bins $\quad 175$

E Beam-constrained $\Upsilon$ Fit in $\left\langle 1 / p_{T}^{\mu}\right\rangle$ Bins $\quad 179$

F Non-beam-constrained $\Upsilon$ Fit in $\left\langle 1 / p_{T}^{\mu}\right\rangle$ Bins $\quad 182$

G Combining Errors $\quad 185$

H QCD Jet Background in CMX-muons 186

I Effects of Fit Window 189

$\mathbf{J} \quad m_{T}, p_{T}^{e}$ and $p_{T}^{\nu}$ Fit Correlations in $W \rightarrow e \nu$ Channel 196 
$\begin{array}{ll}\text { K Using Least Squares to Combine Measurements } & 200\end{array}$

K.0.1 Uncorrelated measurements _. . . . . . . . . . . . . . 201

K.0.2 Two measurements with correlation . . . . . . . . . . . . . 202

K.0.3 Combine statistical uncertainties from $m_{T}, p_{T}^{\mu}$ and $p_{T}^{\nu}$ fits . . 203

$\begin{array}{ll}\text { Bibliography } & 205\end{array}$

$\begin{array}{ll}\text { Biography } & 212\end{array}$ 


\section{List of Tables}

1.1 Summary of fundamental interactions. . . . . . . . . . . . . 2

1.2 A timeline of the discoveries of fundamental particles . . . . . . . . 4

1.3 Shifts in $m_{W}$ when varying $m_{H}$ by $+100 \mathrm{GeV}$ and other input parameters by $+1 \sigma$. The shifts are relative to the value $m_{W}=80.364 \mathrm{GeV}$, which is the result obtained when $m_{H}=100 \mathrm{GeV}$ and PDG central values of other parameters ( like $\alpha_{s}\left(m_{Z}\right)$ etc.) are used. . . . . . . . . 13

1.4 Comparison of required precisions in $m_{t}$ and $m_{W}$ to constrain $m_{H}$ precision to $100 \mathrm{GeV}$. . . . . . . . . . . . . . . . . . . . 14

1.5 W mass measurements at LEP and Tevatron. . . . . . . . . . . . . . 17

2.1 The Fermilab accelerator system. . . . . . . . . . . . . . . . 23

2.2 Some designed accelerator parameters for Tevatron Run II. . . . . . . 24

3.1 Some basic parameters of the COT. . . . . . . . . . . . . . 32

3.2 Some basic parameters of the CDF calorimeter systems. . . . . . . . . 33

5.1 Some datasets used in this $W$ mass analysis. The numbers are after all cuts have been applied. . . . . . . . . . . . . . 54

5.2 Muon candidate requirements. . . . . . . . . . . . . . 55

5.3 Event requirements on $Z$ boson candidates. . . . . . . . . . . . 56

5.4 Event requirements on $W$ boson candidates. . . . . . . . . . . . . 56

5.5 Z $Z$ boson identification for rejection in $W$ boson event selection . . . . 57

5.6 Trigger requirements for $J / \psi$ sample . . . . . . . . . . . . . . . 60

5.7 List of trigger tables for $J / \psi$ sample . . . . . . . . . . . . . . 60

5.8 Muon candidate requirements for $J / \psi$ and $\Upsilon$ samples. . . . . . . . . 61 
6.1 Systematic uncertainty on $m_{W}$ for $m_{T}, p_{T}$ and $\not p_{T}$ fits due to $p_{T}(W)$ model $\left(g_{2}, g_{3}\right.$ and $\left.\alpha_{s}\right)$. . . . . . . . . . . . . . . . . . . . . 69

6.2 Systematic uncertainty on $m_{W}$ for $m_{T}, p_{T}$ and $\not p_{T}$ fits due to photon radiation. ....................... 76

7.1 COT hit resolution for superlayers 1 to $8 \ldots \ldots . \ldots$. . . . . 80

8.1 A list of parameters used to further correct track curvature after alignment. . . . . . . . . . . . . . . . . . 9 90

8.2 Statistical and systematic uncertainties on $\Delta p / p$ from the $J / \psi$ analysis. 100

8.3 Statistical and systematic uncertainties on $\Delta p / p$ from the $\Upsilon$ analysis. 109

8.4 Measured $\Delta p / p$ as a function of run range and instantaneous luminosity, from the beam-constrained $\Upsilon$ mass fit. . . . . . . . . . . . . . 109

8.5 Measured momentum scale deviations from unity . . . . . . . . . . . 110

8.6 Systematic uncertainties on the momentum scale deviation from unity, derived from the non-beam-constrained $\Upsilon$ mass measurement, and the systematics that are common with the $J / \psi$ mass measurement. . . . 110

8.7 Systematic uncertainties on the momentum scale from 1) the combined $J / \psi$ analysis and non-beam-constrained $\Upsilon$ analysis, and 2) the beamconstrained $\Upsilon$ analysis. . . . . . . . . . . . . . . . . . . . 111

8.8 Measured momentum scale deviations from unity from the $200 \mathrm{pb}^{-1}$ $J / \psi$ and $\Upsilon$ analyses. . . . . . . . . . . . . . . . . . . . . 111

8.9 A list of items for the study of momentum scale differences between our new result and the old $200 \mathrm{pb}^{-1}$ result by using $200 \mathrm{pb}^{-1}$ nonbeam-constrained $\Upsilon \rightarrow \mu \mu$ events. . . . . . . . . . . . . . . . . 112

8.10 Results of momentum scale under different cases by using $200 \mathrm{pb}^{-1}$ non-beam-constrained $\Upsilon \rightarrow \mu \mu$ events. . . . . . . . . . . . . . . . 112

9.1 Relative acceptance of $W$ and $Z$ boson decays to pass the $W \rightarrow \mu \nu$ event selection using $\sim 2.2 \mathrm{fb}^{-1} \mathrm{MC}$ samples with some bad MC runs removed. $(\dagger)$ is for HEPG $Z$ mass within $(66,116) \mathrm{GeV} .\left(^{*}\right)$ Assumes other background fractions are the same as those listed in Table 9.3. . 118

9.2 QCD background in CMUP-muons and CMX-muons. . . . . . . . . 127

9.3 Systematic uncertainties on $m_{W}$ due to $W \rightarrow \mu \nu$ background size and shape uncertainties. . . . . . . . . . . . . . . . . . . . . 132 
10.1 Signed shifts in $m_{W}$ due to $1 \sigma$ variations in the recoil response parameters. The shifts are obtained by varying templates with pseudoexperiment histogram fixed. The Monte Carlo statistical uncertainty on the shifts are $\sim 1.6 \mathrm{MeV} \ldots \ldots$. . . . . . . . . . . . . . 138

10.2 Signed shifts in $m_{W}$ due to $1 \sigma$ variations in the recoil resolution parameters. The shifts are obtained by varying templates with pseudoexperiment histogram fixed. The Monte Carlo statistical uncertainty

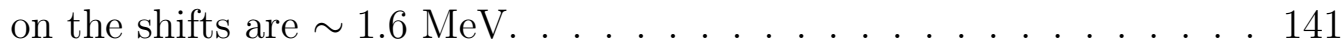

11.1 Systematic and statistical uncertainties on measured $m_{W}$ using $m_{T}$ fit. 149

11.2 Systematic and statistical uncertainties on measured $m_{W}$ using $p_{T}^{\mu}$ fit. 151

11.3 Systematic and statistical uncertainties on measured $m_{W}$ using $p_{T}^{\nu}$ fit. 152

11.4 Cross-checks of fitted $m_{W}$ via $m_{T}$ fit using sub-samples. $\phi_{\mu}>0$ and $\phi_{\mu}<0$ correspond to the upper half and the lower half of the COT, respectively. $\phi_{\mu}^{\text {left }}$ and $\phi_{\mu}^{\text {right }}$ corresponds to the left half $\left(\phi_{\mu} \in\right.$ $(-\pi,-\pi / 2) \cup(\pi / 2, \pi))$ and the right half $\left(\phi_{\mu} \in(-\pi / 2, \pi / 2)\right)$ of the COT. The early and the late data is for runs with run number $<$ and $>222000$, respectively. The $59 \mathrm{MeV}$ systematic uncertainty in $m_{W}^{\mu^{+}}-$ $m_{W}^{\mu^{-}}$is from the statistical uncertainty of COT alignment parameters $a$ and $c$ (see reference [87]). The $\sim 3 \sigma$ difference between early and late runs suggests the best-tuned recoil parameters using all runs does not describe data in early/late run ranges, thus are run-dependent. To get better agreement in $m_{W}$, the recoil parameters need to be retuned for different run ranges. . . . . . . . . . . . . . . . . . 153

11.5 Statistical correlations between $m_{T}, p_{T}^{\mu}$ and $p_{T}^{\nu}$ fits in $W \rightarrow \mu \nu$ channel. The statistical errors are obtained by using Fisher's $z$-transformation. 156

11.6 Comparison of two methods to estimate errors on correlation coefficients between $m_{T}, p_{T}^{\mu}$ and $p_{T}^{\nu}$ fits in $W \rightarrow \mu \nu$ channel. . . . . . . . . 158

11.7 Muon channel $W$ mass fit results and uncertainties from $m_{T}, p_{T}^{\mu}$ and $p_{T}^{\nu}$ distributions. The fit windows are $65-90 \mathrm{GeV} / \mathrm{c}^{2}$ for the $m_{T}$ fit and $32-48 \mathrm{GeV} / \mathrm{c}$ for the $p_{T}^{\mu}$ and $p_{T}^{\nu}$ fits. . . . . . . . . . . 158

J.1 Statistical correlations between $m_{T}, p_{T}^{e}$ and $p_{T}^{\nu}$ fits in $W \rightarrow e \nu$ channel.198 


\section{List of Figures}

1.1 Fundamental particles. . . . . . . . . . . . . . . . . . . 3

1.2 Summary of particle interactions described by the SM, assuming the Higgs boson exists. . . . . . . . . . . . . . . 5 5

1.3 Scalar potential $\mathrm{V}(\phi)(\mu=\lambda=1$ case $) . \phi_{3}$ is the real part of the neutral component of $\phi . \ldots \ldots \ldots$. . . . . . . . . 7

1.4 The one-loop contribution to the $W$ boson mass from top quark and bottom quark. . . . . . . . . . . . . . . . . . 10

1.5 The one-loop contribution to the $W$ boson mass from the Higgs boson. 10

1.6 Higgs mass values excluded at 95\% confidence level by indirect measurements and direct measurements from LEP, Tevatron and LHC experiments ATLAS and CMS. At the 95\% confidence level (CL), LEP experiments exclude $m_{H}<114 \mathrm{GeV} / \mathrm{c}^{2}$; Tevatron experiments exclude $147 \mathrm{GeV} / \mathrm{c}^{2}<m_{H}<179 \mathrm{GeV} / \mathrm{c}^{2}$ and $100 \mathrm{GeV} / \mathrm{c}^{2}<m_{H}<$ $106 \mathrm{GeV} / \mathrm{c}^{2}$; CMS excludes $127 \mathrm{GeV} / \mathrm{c}^{2}<m_{H}<600 \mathrm{GeV} / \mathrm{c}^{2}$; ATLAS excludes $112.9 \mathrm{GeV} / \mathrm{c}^{2}<m_{H}<115.5 \mathrm{GeV} / \mathrm{c}^{2}, 131 \mathrm{GeV} / \mathrm{c}^{2}<m_{H}<$ $238 \mathrm{GeV} / \mathrm{c}^{2}$ and $251 \mathrm{GeV} / \mathrm{c}^{2}<m_{H}<466 \mathrm{GeV} / \mathrm{c}^{2}$. [13] . . . . . . . 11

1.7 The $\Delta \chi^{2}$ as a function of $m_{H}$. This is derived from precision electroweak measurements, assuming the SM is the correct theory of nature. The minimum of the black curve is at $94 \mathrm{GeV}$ with an experimental uncertainty at $68 \% \mathrm{CL}$ of $+29 \mathrm{GeV}$ and $-24 \mathrm{GeV}$ which are derived from $\Delta \chi^{2}=1$ using the black curve. The blue band includes both the experimental and the theoretical uncertainties. The yellow region below $114 \mathrm{GeV}$ is excluded by LEP direct searches at 95\% CL. The yellow region above $127 \mathrm{GeV}$ is excluded by latest LHC searches at $95 \%$ CL. [13] . . . . . . . . . . . . . . . . . 
1.8 Direct $m_{W}$ and $m_{t}$ measurements, indirect constraints from $Z$-pole observables, and the SM theory curves relating $m_{W}$ and $m_{t}$ for fixed values of $m_{H}$ (as of July 2011) [13]. The white band region was excluded by 2011 Tevatron Higgs searches. . . . . . . . . . . . . . . . . 15

1.9 The one-loop contribution to the $W$ boson mass from squarks. . . . . 15

2.1 Overview of the Fermilab accelerator complex. . . . . . . . . . . . 20

2.2 Antiproton production at the target station. The dipole magnets filter anti-protons and direct them to the Debuncher with the rest of the secondary particles sent to the beam dump. . . . . . . . . . . . . 22

2.3 Instantaneous luminosity as a function of time over 11 stores in the week between Sept. 23, 2010 and Oct. 1, 2010 . . . . . . . . . . . 25

2.4 Initial instantaneous luminosity vs. store number. . . . . . . . . . 25

2.5 Integrated luminosity delivered to and acquired by the CDF detector as a function of time. . . . . . . . . . . . . 26

3.1 A schematic view of a section of the CDF detector system. . . . . . 28

3.2 A schematic view of the $\eta$ coverage of the CDF tracking system. . . . 29

3.3 A view of the silicon detector along the beam direction $\hat{z}$. . . . . . 30

3.4 Structure of SL2 and three COT cells are shown. Other superlayers are similar except for the taper. Each cell consists of 12 sense wires, 13 potential wires, 4 shaper wires and one Au-mylar cathode field panel on both sides of the sense/potential wire plane. . . . . . . . . . . . 31

3.5 A view of $1 / 6$ section of the COT. . . . . . . . . . . . . . 32

3.6 The structure of a typical CEM wedge. . . . . . . . . . . . . . . . . 34

3.7 Schematic view of typical CMU wedge. . . . . . . . . . . . . . . 36

3.8 A view of CMU module (left) and CMP module (right) in $r-\phi$ plane. 36

3.9 Coverage of CMU, CMP and CMX in $\phi-\eta$ plane. . . . . . . . . . . . 37

3.10 Diagram of the CDF dataflow [44]. . . . . . . . . . . . . . 39

3.11 Block diagram of the Level 1 and the Level 2 trigger systems at CDF [44]. 40

4.1 Leading order production and leptonic decay of a $W$ boson at the Tevatron. ......................... . . . 44 
4.2 Axial view of a $W \rightarrow \mu \nu$ event in the CDF detector. The hadronic recoil deposits energy in calorimeter, the muon track leaves hits in the muon chambers and the neutrino escapes detection [59]. . . . . . . . 44

$4.3 W^{+}$decay in the center of mass frame. . . . . . . . . . . . . 45

4.4 Illustrations of $m_{T}$ (top) and $p_{T}^{\mu}$ (bottom) spectra to $p_{T}^{W}$ and detector effects. Shaded area is for both finite detector resolution and $p_{T}^{W} \neq$ 0 ; blue points is for finite detector resolution with $p_{T}^{W}=0$; black histrogram is for perfect detector resolution with $p_{T}^{W} \neq 0$ [59]. . . . .

6.1 Variations of fitted $m_{W}$ difference from the default using $m_{T}$ distributions over 20 pairs of CTEQ6 error PDFs. The difference in $M_{W}$ is between each error PDF and the default CTEQ6M. . . . . . . . . . 65

6.2 Variations of fitted $m_{W}$ difference from the default using $p_{T}^{\mu}$ distributions over 20 pairs of CTEQ6 error PDFs. The difference in $M_{W}$ is between each error PDF and the default CTEQ6M. . . . . . . . . . 66

6.3 Variations of fitted $m_{W}$ difference from the default using $p_{T}^{\nu}$ distributions over 20 pairs of CTEQ6 error PDFs. The difference in $M_{W}$ is between each error PDF and the default CTEQ6M. . . . . . . . . . .

6.4 Leading order QCD processes contributing to the production of $W$ boson. The first diagram is for quark-gluon fusion, the second diagram is for gluon initial state radiation, the rest two diagrams are for associated $W$-gluon production $[45] \ldots \ldots$. . . . . . . .

6.5 Standard error ellipse for estimator $\hat{\theta}_{i}$ and $\hat{\theta}_{j}$ which are anti-correlated

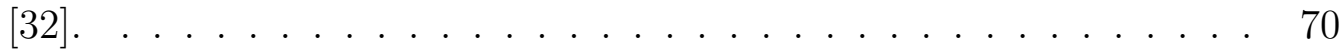

6.6 RESBOS-generated $W$ boson $p_{T}$ spectrum for $g_{2}=0.48 \mathrm{GeV}^{2}, 0.68$

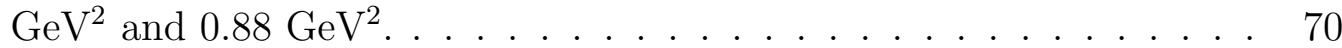

6.7 $Z$ boson $p_{T}$ spectrum using $Z \rightarrow \mu \mu$ events. This spectrum, together with the $Z$ boson $p_{T}$ spectrum using $Z \rightarrow$ ee events, is used to fit for $g_{2} .71$

6.8 The four plots on the top show, for the $W^{+} \rightarrow \mu^{+} \nu+n \gamma$ process, the comparison of $\log _{10}\left(y_{\gamma}\right), \log _{10}(\Delta R(l \gamma)), \log _{10}\left(E_{\gamma} / \mathrm{GeV}\right)$ and $n_{\gamma}$, respectively. The $\Delta R$ is computed with respect to the muon. The bottom plot shows the comparison of invariant mass of all final state particles using OLD HORACE and PHOTOS. [66] . . . . . . . . . . . 75

7.1 COT hit residuals for each superlayer, extracted from $Z \rightarrow \mu \mu$ data tracks. . . . . . . . . . . . . . . . . . . 81 
7.2 Variation of the muon identification efficiency as a function of $u_{\|}$, the recoil componenent in the direction of the muon. . . . . . . . . . . .

7.3 Muon acceptance efficiency as a function of $\cot \theta$ (left) and $\phi$ (right). The top (bottom) row is for CMUP (CMX) . . . . . . . . . . 84

8.1 Flow chart of momentum scale (or $\Delta p / p)$ measurement. . . . . . . . 87

8.2 A cosmic ray event recorded by the CDF detector. The green line shows the trajectory of the cosmic ray muon and the red arrow indicates the direction of the vector sum of missing transverse energy $E_{T}$ in the calorimeter. Particle ID 13 represents muon. . . . . . . . . . .

8.3 Illustration of global azimuthal shift $(R \Delta \phi)$ and local tilt around cell center $(\Delta \tau)$ in the COT alignment $[8] . \ldots \ldots \ldots$

8.4 The $\cot \theta$ dependence (left) and the $\phi$ dependence (right) of $\Delta(E / p)$. The red triangles are before track correction and the blue circles are after track correction. . . . . . . . . . . . . . . . . . . . . . 89

8.5 The $p_{T}^{J / \psi}\left(p_{T}^{\mu \mu}\right)$ distributions before (left) and after (right) tuning. . . 92

8.6 The distributions of the sum of $\left(q / p_{T}\right)$ over two decay muons before (left) and after (right) tuning. . . . . . . . . . . . 93

8.7 Kinematics in the quarkonium rest frame. . . . . . . . . . . . 93

8.8 Data and simulation distributions of the muon transverse momentum

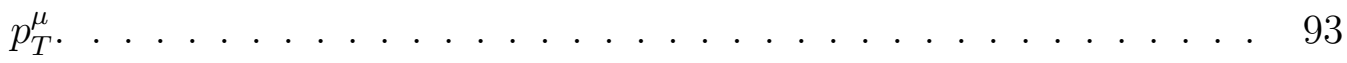

8.9 Data and simulation distributions of the average unsigned curvature of the two muons. . . . . . . . . . . . . . . . . . . 93

8.10 Data and simulation distributions of the $J / \psi$ momentum in $z$ direction. 94

8.11 Data and simulation distributions of $p_{z}$ of the decay muons. . . . . . 94

8.12 Data and simulation distributionss of $\cot \theta$ of the decay muons. . . . 94

8.13 Data and simulation distributions of $\Delta \cot \theta$ of the decay muons. . . . 94

8.14 Illustration of events used to study non-uniform magnetic field within

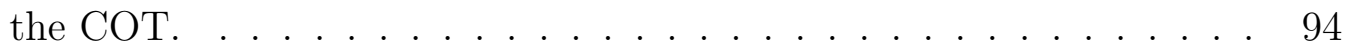

8.15 Fitted $\Delta p / p$ as a function of $\cot \theta$ with $|\Delta \cot \theta|<0.1$, before corrections (left) and after corrections (right), fitted to a parabola. . . . . 9 95

8.16 Illustration of events used to study COT misalignment effects. . . . . 96 
8.17 Fitted $\Delta p / p$ as a function of $\Delta \cot \theta$, before corrections (left) and after corrections (right), fitted to a parabola. . . . . . . . . . . . 97

8.18 Fitted $\Delta p / p$ as a function of $\left\langle 1 / p_{T}^{\mu}\right\rangle$, fitted to a straight line. . . . 97

8.19 Dimuon mass spectrum with a fit of three Gaussian distributions and a linear background . . . . . . . . . . . . . . . . . . 101

8.20 Comparison of data and Monte Carlo of the $\Upsilon(1 \mathrm{~S}) p_{T}$ distribution. . 102

8.21 Comparison of data and Monte Carlo of the $\Upsilon(1 \mathrm{~S}) p_{z}$ distribution. . . 102

8.22 Comparison of data and Monte Carlo of the muon candidate $p_{T}$ distribution. . . . . . . . . . . . . . . . . . 103

8.23 Comparison of data and Monte Carlo of the muon candidate $p_{z}$ dis-

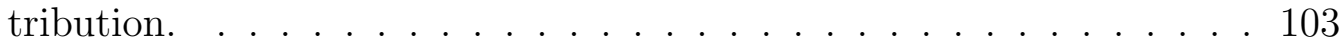

8.24 Comparison of data and Monte Carlo of the average muon inverse transverse momentum distribution. . . . . . . . . . . . . 103

8.25 Comparison of data and Monte Carlo of the summed muon inverse transverse momentum distribution. . . . . . . . . . . . . . . . . 103

8.26 Data and simulation distributions of $\cot \theta$ of the decay muons. . . . . 103

8.27 Data and simulation distributions of $\Delta \cot \theta$ of the decay muons. . . . 103

8.28 Fitted $\Delta p / p$ as a function of $\Delta \cot \theta$ for beam-constrained $\Upsilon_{\mathrm{s}}$, before corrections, fitted to a parabola. . . . . . . . . . . . . . . . 104

8.29 Fitted $\Delta p / p$ as a function of $\Delta \cot \theta$ for beam-constrained $\Upsilon_{\mathrm{s}}$, after corrections, fitted to a parabola. . . . . . . . . . . . . . . 104

8.30 Fitted $\Delta p / p$ as a function of $\Delta \cot \theta$ for non-beam-constrained $\Upsilon_{\mathrm{s}}$, before corrections, fitted to a parabola. . . . . . . . . . 105

8.31 Fitted $\Delta p / p$ as a function of $\Delta \cot \theta$ for non-beam-constrained $\Upsilon_{\mathrm{S}}$, after corrections, fitted to a parabola. . . . . . . . . . . . . 105

8.32 Fitted $\Delta p / p$ as a function of $\left\langle 1 / p_{T}^{\mu}\right\rangle$ using beam-constrained $\Upsilon \rightarrow \mu \mu$ events, fitted to a straight line. The slope is consistent with zero. The material scale from the $J / \psi$ analysis is included in the $\Upsilon$ simulation. This shows that the $\Upsilon$ data are consistent with the $J / \psi$ data with respect to the ionization energy loss in the detector material. . . . . . 106 
8.33 Fitted $\Delta p / p$ as a function of $\left\langle 1 / p_{T}^{\mu}\right\rangle$ using non-beam-constrained $\Upsilon \rightarrow$ $\mu \mu$ events, fitted to a straight line. The slope is consistent with zero. The material scale from the $J / \psi$ analysis is included in the $\Upsilon$ simulation. This shows that the $\Upsilon$ data are consistent with the $J / \psi$ data with respect to the ionization energy loss in the detector material. . . 107

8.34 The invariant mass distribution with the best fit simulated template and the extracted momentum scale using beam-constrained track quantities. . . . . . . . . . . . . . . . . . . . . . 108

8.35 The invariant mass distribution with the best fit simulated template and the extracted momentum scale using non-beam-constrained track quantities. . . . . . . . . . . . . . . . . . 108

8.36 The fitted $Z \rightarrow \mu \mu$ mass using the COT momentum scale derived from $J / \psi \rightarrow \mu \mu$ and $\Upsilon \rightarrow \mu \mu$ mass fits. The fitting range is from 83.0 GeV to $99.0 \mathrm{GeV}$. . . . . . . . . . . . . . . . . . . . . . 114

9.1 Comparison of the impact parameter $d_{0}$ distributions. The red points are for the $W$ boson candidate sample, the black histrogram is for $W$ boson simulation from DukeSim, and the blue points are for a DIFenriched data sample obtained by anti-selecting on the $N_{\text {trans }}$. We use $0.2<d<0.5 \mathrm{~cm}$ to obtain DIF sample. . . . . . . . . . . . . . 122

9.2 Number of transitions $N_{\text {trans }}$ as a function of $\chi^{2} / d f$ for $Z \rightarrow \mu \mu$ data signal (red points) and DIF muons from $W$ sideband (blue points). The black line is $N_{\text {trans }}=30+2 \cdot \chi^{2} / d f$. . . . . . . . . . . . . . . 124

9.3 An illustration of the three-layer structure used in the QCD jet background $\mathrm{NN}$ analysis. . . . . . . . . . . . . . 125

9.4 The log distributions of trkIso (left) and hadIso (right) for $Z$ muons of CMUP type. The red histogram is for $Z$ CMUP muons from MC while the blue histogram is for $Z$ CMUP muons from data. The MC spectrum in the right plot has been linearly transformed to match mean and rms of the data spectrum. . . . . . . . . . . . . . . . . . . 128

9.5 The log distributions of trkIso (left) and hadIso (right) for $Z$ muons of CMUP type. The red histogram is for $Z \mathrm{MC}$ muon while the black histogram is for QCD jet events from $W$ boson candidates with $p_{T}^{\nu}<$ $10 \mathrm{GeV}$ and $u_{T}<45 \mathrm{GeV}$. . . . . . . . . . . . . . . . . . . . . . 128

9.6 The NN output distributions for $Z$ MC CMUP-muons, $Z$ data CMUPmuons and QCD jet events (left), and $W$ MC CMUP-muons, $W$ data CMUP-muons and QCD control sample (right). . . . . . . . . . . . 129 
9.7 $\chi^{2}$ as a function of the fraction of QCD jet background for $Z$ CMUP muons (left) and $W$ CMUP muons (right). . . . . . . . . . . . . 129

$9.8 m_{T}$ distributions for all backgrounds in $W \rightarrow \mu \nu$ channel. Each background is normalized to its corresponding fraction in Table 9.3. . . . 131

$9.9 p_{T}$ distribtions for all backgrounds in $W \rightarrow \mu \nu$ channel. Each background is normalized to its corresponding fraction in Table 9.3. . . . 131

$9.10 \not p_{T}$ distribtions for all backgrounds in $W \rightarrow \mu \nu$ channel. Each background is normalized to its corresponding fraction in Table 9.3. . . . 132

10.1 Illustration of $u_{\|}$and $u_{\perp}$ for the decomposition of the recoil momentum $\vec{u}_{T}$ in the transverse plane for a typical $W$ boson decay. The $u_{\|}$ direction is defined to be along $\vec{p}_{T}^{\mu}$, the $u_{\perp}$ direction is perpendicular

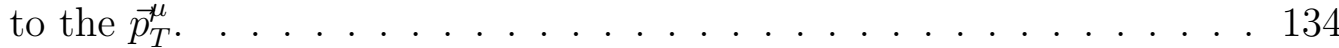

10.2 Grids for mean EM (left) and hadronic (right) energy in calorimeter towers surrounding the muon track using $W \rightarrow \mu \nu$ data events. . . . 135

10.3 The $\vec{p}_{T}$ vectors of the two leptons in $Z \rightarrow l^{+} l^{-}$events. The $\eta$ axis is parallel to the $\vec{p}_{T}(l l)$ direction and the $\xi$ axis is perpendicular to it. . 136

10.4 Mean variation of $0.65 \cdot p_{T}^{\eta}(Z)+u_{T}^{\eta}$ as a function of $p_{T}^{Z \rightarrow l l}$ using $Z \rightarrow \mu \mu$ events. This plot, together with the corresponding plot using $Z \rightarrow e e$ events, is used in tuning recoil scale parameters. The red histograms are from DukeSim while the blue points are from data. The factor of 0.65 is chosen such that the mean value of $0.65 p_{T}^{\eta}(Z)+u_{T}^{\eta}$ is approximately $0 \ldots \ldots \ldots \ldots \ldots \ldots$

10.5 RMS variation of $0.65 \cdot p_{T}^{\eta / \xi}(Z)+u_{T}^{\eta / \xi}$ as a function of $p_{T}^{Z \rightarrow l l}$ using $Z \rightarrow \mu \mu$ events. The top plot, together with the corresponding plot using $Z \rightarrow e e$ events, is used to tune jet energy resolution parameter $s_{\text {had }}$ and the underlying event scaling parameter $N_{V}$. The bottom plot, together with the corresponding plot using $Z \rightarrow e e$ events, is used to tune the jet angular resolution parameters $\alpha, \beta$ and $\gamma$. The data are shown in blue points and the simulation is shown in red histogram. . 142

10.6 Comparison of $u_{\|}$between data (blue points) and simulation (red histogram) using $W \rightarrow \mu \nu$ events. The quoted uncertainties are statistical only. . . . . . . . . . . . . . . . . . . . . . . . . . . . 143

10.7 Comparison of $u_{\perp}$ distribution between data (blue points) and simulation (red histogram) using $W \rightarrow \mu \nu$ events. The quoted uncertainties are statistical only. . . . . . . . . . . . . . . . . . . . 144 
10.8 Comparison of $u_{T}$ distribution between data (blue points) and simulation (red histogram) using $W \rightarrow \mu \nu$ events. . . . . . . . . . . . . . . 144

10.9 Comparison of $\Delta \phi_{u, \mu}$ between data (blue points) and simulation (red histogram) using $W \rightarrow \mu \nu$ events. . . . . . . . . . . . . . . . . 145

11.1 Transverse mass templates used to extract the $W$ boson mass $m_{W}$. $\quad 148$

11.2 The $m_{T}$ distribution for $W$ boson decays to $\mu \nu$ with data in blue points and simulation in solid line. . . . . . . . . . . . . . . 149

11.3 The $p_{T}^{\mu}$ distribution for $W$ boson decays to $\mu \nu$ with data in blue points and simulation in solid line. . . . . . . . . . . . . . . . . . . 150

11.4 The $\mathbb{E}_{T}$ distribution for $W$ boson decays to $\mu \nu$ with data in blue points and simulation in solid line. . . . . . . . . . . . . . . . . . . 152

11.5 The shifts in measured $m_{W}$, from $W \rightarrow \mu \nu$ channel, for variations in the lower (left) and upper (right) edges of $m_{T}, p_{T}$ and $\not p_{T}$ fit ranges. The points describe the shifts in $m_{W}$ relative to the default values. The error bars are the expected one standard deviations obtained from simulation pseudoexperiments for the corresponding fitting window. . 160

11.6 Correlations between $m_{T}, p_{T}^{\mu}$ and $p_{T}^{\nu}$ fits. . . . . . . . . . . . . 161

11.7 Monte Carlo study of the errors on correlation coefficients between $m_{T}, p_{T}^{\mu}$ and $p_{T}^{\nu}$ fits. . . . . . . . . . . . . . . . . 162

11.8 Comparison of the $W$ boson mass measurement from $m_{T}, p_{T}^{\mu}$ and $p_{T}^{\nu}$ fits. The uncertainties are statistical only. The dark band is for the new world-average $m_{W}$ and its associated total uncertainty. . . . . . 163

12.1 Recent and past $W$ boson mass measurements. Combining muon channel result with electron channel result, CDF reaches a precision of $19 \mathrm{MeV}$, which is significantly better than all previous measurements. 166

12.2 Updated $m_{W}$ vs. $m_{t}$ plot with the latest CDF and D $\varnothing m_{W}$ measurements. The green region is SM-allowed region and the yellow region has been excluded by LHC experiments. . . . . . . . . . . . . . . . . 167

12.3 Projection of measurement precision of the $W$ boson mass at CDF and $\mathrm{D} \varnothing$ as a function of integrated luminosity $\mathcal{L}$. The red curve is obtained by assuming the theoretical uncertainties from PDF (10 $\mathrm{MeV}$ ) and QED (4 MeV) remain unchanged, while other systematic uncertainties and statistical uncertainy scale as $1 / \sqrt{\mathcal{L}}$. . . . . . . . 168 
A.1 Variations of fitted $m_{W}$ difference from the default using $W \rightarrow e \nu m_{T}$ distributions over 20 pairs of CTEQ6 error PDFs. The difference in $m_{W}$ is between each error PDF and the default CTEQ6M. . . . . . . 169

A.2 Variations of fitted $m_{W}$ difference from the default using $W \rightarrow e \nu p_{T}^{e}$ distributions over 20 pairs of CTEQ6 error PDFs. The difference in $m_{W}$ is between each error PDF and the default CTEQ6M. . . . . . . 170

A.3 Variations of fitted $m_{W}$ difference from the default using $W \rightarrow e \nu E_{T}$ distributions over 20 paris of CTEQ6 error PDFs. The difference in $m_{W}$ is between each error PDF and the default CTEQ6M. . . . . . . 170

B.1 $Z / \gamma$ interference contribution to the $Z$ production from RESBOS. . . . 172

D.1 Fitted $\Delta p / p$ of the first $\left\langle 1 / p_{T}^{\mu}\right\rangle$ bin in Figure 8.18. . . . . . . . . . 175

D.2 Fitted $\Delta p / p$ of the second $\left\langle 1 / p_{T}^{\mu}\right\rangle$ bin in Figure 8.18. . . . . . . . . 175

D.3 Fitted $\Delta p / p$ of the third $\left\langle 1 / p_{T}^{\mu}\right\rangle$ bin in Figure 8.18. . . . . . . . . 176

D.4 Fitted $\Delta p / p$ of the fourth $\left\langle 1 / p_{T}^{\mu}\right\rangle$ bin in Figure 8.18. . . . . . . . . 176

D.5 Fitted $\Delta p / p$ of the fifth $\left\langle 1 / p_{T}^{\mu}\right\rangle$ bin in Figure 8.18 . . . . . . . . 176

D.6 Fitted $\Delta p / p$ of the sixth $\left\langle 1 / p_{T}^{\mu}\right\rangle$ bin in Figure 8.18. . . . . . . . . . 176

D.7 Fitted $\Delta p / p$ of the seventh $\left\langle 1 / p_{T}^{\mu}\right\rangle$ bin in Figure 8.18. . . . . . . . 176

D.8 Fitted $\Delta p / p$ of the eighth $\left\langle 1 / p_{T}^{\mu}\right\rangle$ bin in Figure 8.18 . . . . . . . . 176

D.9 Fitted $\Delta p / p$ of the ninth $\left\langle 1 / p_{T}^{\mu}\right\rangle$ bin in Figure 8.18 . . . . . . . . 177

D.10 Fitted $\Delta p / p$ of the tenth $\left\langle 1 / p_{T}^{\mu}\right\rangle$ bin in Figure 8.18 . . . . . . . . 177

D.11 Fitted $\Delta p / p$ of the eleventh $\left\langle 1 / p_{T}^{\mu}\right\rangle$ bin in Figure 8.18 . . . . . . . . . 177

D.12 Fitted $\Delta p / p$ of the twelfth $\left\langle 1 / p_{T}^{\mu}\right\rangle$ bin in Figure 8.18. . . . . . . . . . 177

D.13 Fitted $\Delta p / p$ of the thirteenth $\left\langle 1 / p_{T}^{\mu}\right\rangle$ bin in Figure 8.18. . . . . . . . 177

D.14 Fitted $\Delta p / p$ of the fourteenth $\left\langle 1 / p_{T}^{\mu}\right\rangle$ bin in Figure 8.18. . . . . . . 177

D.15 Fitted $\Delta p / p$ of the fifteenth $\left\langle 1 / p_{T}^{\mu}\right\rangle$ bin in Figure 8.18. . . . . . . . 178

E.1 Fitted $\Delta p / p$ of the first $\left\langle 1 / p_{T}^{\mu}\right\rangle$ bin in Figure 8.18. . . . . . . . . 179

E.2 Fitted $\Delta p / p$ of the second $\left\langle 1 / p_{T}^{\mu}\right\rangle$ bin in Figure 8.18. . . . . . . . 179

E.3 Fitted $\Delta p / p$ of the third $\left\langle 1 / p_{T}^{\mu}\right\rangle$ bin in Figure 8.18. . . . . . . . 180 
E.4 Fitted $\Delta p / p$ of the fourth $\left\langle 1 / p_{T}^{\mu}\right\rangle$ bin in Figure 8.18. . . . . . . . . 180

E.5 Fitted $\Delta p / p$ of the fifth $\left\langle 1 / p_{T}^{\mu}\right\rangle$ bin in Figure 8.18. . . . . . . . . . 180

E.6 Fitted $\Delta p / p$ of the sixth $\left\langle 1 / p_{T}^{\mu}\right\rangle$ bin in Figure 8.18. . . . . . . . . . . 180

E.7 Fitted $\Delta p / p$ of the seventh $\left\langle 1 / p_{T}^{\mu}\right\rangle$ bin in Figure 8.18. . . . . . . . 180

E.8 Fitted $\Delta p / p$ of the eighth $\left\langle 1 / p_{T}^{\mu}\right\rangle$ bin in Figure 8.18. . . . . . . . . . 180

E.9 Fitted $\Delta p / p$ of the ninth $\left\langle 1 / p_{T}^{\mu}\right\rangle$ bin in Figure 8.18. . . . . . . . . . 181

F.1 Fitted $\Delta p / p$ of the first $\left\langle 1 / p_{T}^{\mu}\right\rangle$ bin in Figure 8.18. . . . . . . . . . 182

F.2 Fitted $\Delta p / p$ of the second $\left\langle 1 / p_{T}^{\mu}\right\rangle$ bin in Figure 8.18. . . . . . . . . 182

F.3 Fitted $\Delta p / p$ of the third $\left\langle 1 / p_{T}^{\mu}\right\rangle$ bin in Figure 8.18. . . . . . . . . 183

F.4 Fitted $\Delta p / p$ of the fourth $\left\langle 1 / p_{T}^{\mu}\right\rangle$ bin in Figure 8.18. . . . . . . . 183

F.5 Fitted $\Delta p / p$ of the fifth $\left\langle 1 / p_{T}^{\mu}\right\rangle$ bin in Figure 8.18. . . . . . . . . 183

F.6 Fitted $\Delta p / p$ of the sixth $\left\langle 1 / p_{T}^{\mu}\right\rangle$ bin in Figure 8.18. . . . . . . . . 183

F.7 Fitted $\Delta p / p$ of the seventh $\left\langle 1 / p_{T}^{\mu}\right\rangle$ bin in Figure 8.18. . . . . . . . 183

F.8 Fitted $\Delta p / p$ of the eighth $\left\langle 1 / p_{T}^{\mu}\right\rangle$ bin in Figure 8.18. . . . . . . . . 183

F.9 Fitted $\Delta p / p$ of the ninth $\left\langle 1 / p_{T}^{\mu}\right\rangle$ bin in Figure 8.18. . . . . . . . . 184

H.1 The log distributions of trkIso (left) and hadIso (right) for $Z$ muons of CMX type. The red histogram is for $Z$ CMX muons from MC while the blue histogram is for $Z$ CMX muons from data. . . . . . . . . . . 187

H.2 The log distributions of trkIso (left) and hadIso (right) for $Z$ muons of CMX type. The red histogram is for $Z$ MC muon while the black histogram is for QCD jet events from $W$ boson candidates with $p_{T}^{\nu}<$ $10 \mathrm{GeV}$ and $u_{T}<45 \mathrm{GeV} \ldots \ldots . \ldots . . \ldots 187$

H.3 The NN output distributions for $Z$ MC CMX-muons, $Z$ data CMXmuons and QCD jet events (left), and $W$ MC CMX-muons, $W$ data CMX-muons and QCD control sample (right). . . . . . . . . . . 188

H.4 $\chi^{2}$ as a function of the fraction of QCD jet background for $Z$ CMX muons (left) and $W$ CMX muons (right). . . . . . . . . . . . . . 188 
I.1 The statistical variations in measured $m_{W}$, using simulated $W \rightarrow \mu \nu$ events, as the lower edge of fit range in $m_{T}$ spectrum changes from 70 $\mathrm{GeV}$ to $60 \mathrm{GeV}$ with a step size $-1 \mathrm{GeV}$ while the upper edge fixed at $90 \mathrm{GeV}$. . . . . . . . . . . . . . . . . . . 190

I.2 The statistical variations in measured $m_{W}$, using simulated $W \rightarrow \mu \nu$ events, as the upper edge of fit range in $m_{T}$ spectrum changes from $85 \mathrm{GeV}$ to $95 \mathrm{GeV}$ with a step size $+1 \mathrm{GeV}$ while the lower edge fixed at $65 \mathrm{GeV}$. . . . . . . . . . . . . . . . . . 191

I.3 The statistical variations in measured $m_{W}$, using simulated $W \rightarrow \mu \nu$ events, as the lower edge of fit range in $p_{T}^{\mu}$ spectrum changes from 35 $\mathrm{GeV}$ to $30 \mathrm{GeV}$ with a step size $-0.5 \mathrm{GeV}$ while the upper edge fixed at $48 \mathrm{GeV}$. . . . . . . . . . . . . . . . . . . . . . . . 192

I.4 The statistical variations in measured $m_{W}$, using simulated $W \rightarrow \mu \nu$ events, as the upper edge of fit range in $p_{T}^{\mu}$ spectrum changes from $45.5 \mathrm{GeV}$ to $50.5 \mathrm{GeV}$ with a step size $+0.5 \mathrm{GeV}$ while the lower edge fixed at $32 \mathrm{GeV}$. . . . . . . . . . . . . . . . . . 193

I.5 The statistical variations in measured $m_{W}$, using simulated $W \rightarrow \mu \nu$ events, as the lower edge of fit range in $p_{T}^{\nu}\left(p_{T}^{\prime}\right)$ spectrum changes from $35 \mathrm{GeV}$ to $30 \mathrm{GeV}$ with a step size $-0.5 \mathrm{GeV}$ while the upper edge fixed at $48 \mathrm{GeV}$. . . . . . . . . . . . . . . . . . . . . . . 194

I.6 The statistical variations in measured $m_{W}$, using simulated $W \rightarrow \mu \nu$ events, as the upper edge of fit range in $p_{T}^{\nu}\left(\not \not_{T}\right)$ spectrum changes from $45.5 \mathrm{GeV}$ to $50.5 \mathrm{GeV}$ with a step size $+0.5 \mathrm{GeV}$ while the lower edge fixed at $32 \mathrm{GeV}$. . . . . . . . . . . . . . . . . . 195

J.1 Correlations between $m_{T}, p_{T}^{e}$ and $p_{T}^{\nu}$ fits in $W \rightarrow e \nu$ channel. . . . . 197

J.2 Monte Carlo study of the errors on correlation coefficients between $m_{T}, p_{T}^{\mu}$ and $p_{T}^{\nu}$ fits in $W \rightarrow \mu \nu$ channel. . . . . . . . . . . . . . . 199

K.1 Variation of combined statistical uncertainty from $m_{T}, p_{T}^{\mu}$ and $p_{T}^{\nu}$ fits due to the $1 \sigma$ statistical uncertainty of correlation coefficients. . . . . 204 


\section{Acknowledgements}

I would like to thank all the people who have helped me over the past few years.

First of all, I would like to thank my advisor Prof. Ashutosh Kotwal for his encouragement and support. I admire his energy and enthusiasm for high energy physics as well as his deep insight of physics problems. Nothing could have been achieved in this challenging $W$ mass measurement without his guidance and help.

I would also like to thank Electroweak Group leaders Prof. Mark Lancaster and Prof. Larry Nodulman, and $W$ mass group convenors Oliver Stelzer-Chilton, Emily Nurse, Ilija Bizjak and Bo Jayatilaka for their wonderful leadership. Special thanks to Oliver Stelzer-Chilton for helping me get started on the momentum scale measurement ever since I joined the $W$ mass group. Special thanks also go to Chris Hays for his challenging questions and to Rob Roser for his support during my application to the URA Visiting Scholar Program at Fermilab.

I would like to thank Prof. Al Goshaw, Mark Kruse, Steffen Bass and Ronen Plesser for squeezing time out of their busy schedules to serve on my thesis committee. I am also grateful to the graduate students at Duke and at Fermilab: Jianrong Deng, Dean Hidas, Ravi Shekhar, Joel Greenberg, Seth Henshaw, Callan Kristine, Adam Sokolow, Huidong Xu, Dong Liu, Fang Yuan, Jianming Zhang, Tingjun Yang, Jian Tang, Qiuguang Liu, Hao Liu, Kai Yi, Fan Yang, Jie Chen, Guo Chen, Dun Zhang, Hang Yin, Xuebing Bu, Yunhe Xie, Pengfei Ding, Zhenyu Ye and Weigang Geng. It's a great and memorable experience for me to have my PhD study at Duke and 
at Fermilab.

Finally, I want to thank my family for their patience, encouragement and love in years of my PhD study. I couldn't have got this far without your support! 


\section{Introduction}

\subsection{The Standard Model}

Particle physics is a branch of physics studying the fundamental particles and their mutual interactions. Particle physics is also known as high energy physics (HEP) since some fundamental particles do not occur under normal circumstances and they can only be created at high energies. In the past century, many particles have been found and people have been gaining a much deeper understanding of what the fundamental constituents are, especially with the help of acceleration technology. A rule which tries to explain all the particles and their interactions we have observed so far is developed into a currently well-known theory - the Standard Model (SM) [1] [2] [3].

The SM is a relativistic quantum field theory in which the dynamics is generated from the assumption of local gauge invariance. The SM is a renormalizable theory which means divergences can be absorbed into parameters such as masses and coupling strengths. Currently, the SM encompasses two sectors: the electroweak sector and the strong interaction sector. The electroweak sector of the SM unifies both elec- 
tromagnetic (E\&M) interaction and weak interaction by exchanging $\gamma$ and $W^{ \pm} / Z$, respectively. The strong interaction sector is described by a theory named Quantum Chronodynamics (QCD), with the exchange of gluon as its interaction mechanism. Table 1.1 summarizes the above three fundamental interactions together with the gravitational interaction, which is much weaker than the other interactions and not included in the SM.

Table 1.1: Summary of fundamental interactions.

\begin{tabular}{lcccc}
\hline \hline Interaction & Boson & Distance $(\mathrm{m})$ & Source & Theory \\
\hline E\&M & photon $(\gamma)$ & $\infty$ & electric charge & Electroweak \\
\hline Weak & $W^{ \pm}, Z$ & $\sim 10^{-18}$ & flavor & Electroweak \\
\hline Strong & gluon $(\mathrm{g})$ & $\leq 10^{-15}$ & color charge & QCD \\
\hline Gravity & graviton $(\mathrm{G})$ & $\infty$ & mass & General Relativity \\
\hline \hline
\end{tabular}

Fundamental particles described by the SM are classified into two categories: fermions and bosons. The fundamental constituents of matter, leptons and quarks, are fermions; while the interactions of fermions are described by mediating force carriers - bosons. Some bosons, like $W^{ \pm}, Z$ and the gluons, can even have self interactions. Fermions can be further divided into three generations such that particles between generations differ only by their masses while all interactions and quantum numbers are the same. Till now, it still puzzles the HEP community why generations exist. Figure 1.1 shows the classification of fundamental particles together with their intrinsic properties like mass, charge and spin.

Considering the three degrees of freedom each quark has in its color space, there are altogether $6_{(u, d, c, s, t, b)} \times 3_{(\text {color })} \times 2_{(q \bar{q})}=36$ quarks in the SM, where anti-quarks are also included. For the number of leptons, there are only $6_{\left(e, \nu_{e}, \mu, \nu_{\mu}, \tau, \nu_{\tau}\right)} \times 2_{(\overline{l l})}=12$ since leptons do not have color. Together with the 13 bosons $\left(\gamma, W^{ \pm}, Z, 8\right.$ gluons 


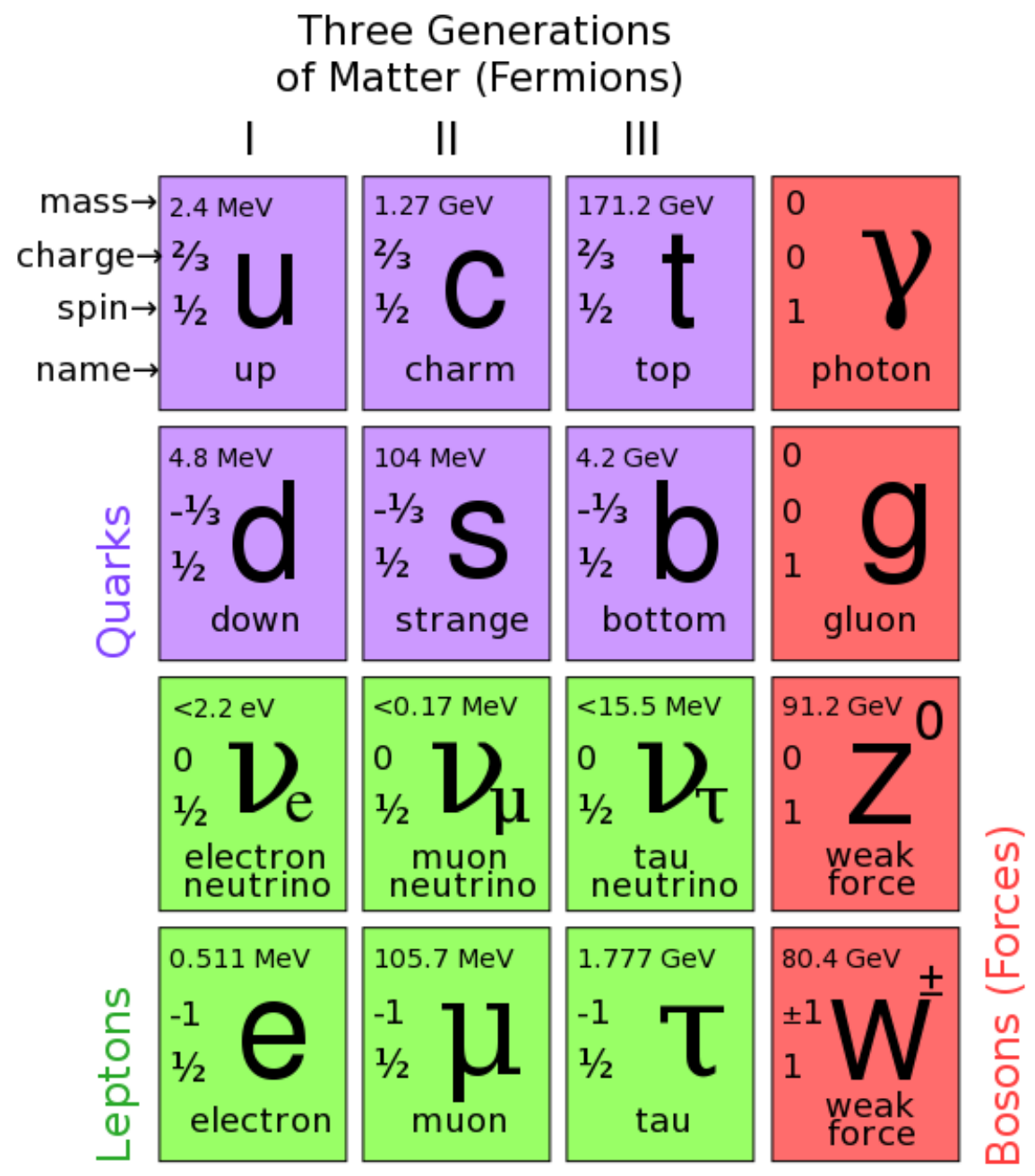

Figure 1.1: Fundamental particles.

and one neutral Higgs boson) required by the minimal SM, the complete particle spectrum is obtained. With those 61 fundamental particles included, 26 parameters [5] which include particle masses, coupling strengths and mixing angles are needed to feed into the SM to make it completely predictive. As the only elementary particle physics theory that has been verified experimentally, the SM is quite successful in its predictions. One famous example is its successful prediction of the neutral weak force carrier - the $Z$ boson and, the charged weak force carrier - the $W$ boson, which 
are both discovered on UA1 and UA2 experiments at CERN in 1980s. A timeline of the discoveries of fundamental particles is summarized in Table 1.2.

Table 1.2: A timeline of the discoveries of fundamental particles

\begin{tabular}{lcc}
\hline \hline Time & Particle & Observer (Postulator) \\
\hline 1897 & $e$ & J.J. Thomson et al. \\
\hline 1936 & $\mu$ & C.D. Anderson et al. \\
\hline 1956 & $\nu_{e}$ & F. Reines et al. \\
\hline 1962 & $\nu_{\mu}$ & G. Danby et al. \\
\hline 1964 & $u, d, s$ & M. Gell-Mann et al. \\
\hline 1974 & $c$ & S. Ting and B. Richter et $a l$. \\
\hline 1975 & $\tau$ & M. Perl et al. \\
\hline 1977 & $b$ & L.M. Ledermann et al. \\
\hline 1983 & $W, Z$ & UA1 and UA2 Collaborations at LEP \\
\hline 1995 & $t$ & CDF and D $\varnothing$ Collaborations at FNAL \\
\hline 2000 & $\nu_{\tau}$ & DONUT Collaboration \\
\hline \hline
\end{tabular}

Despite of its great success, however, there is still one postulated but undiscovered particle, the Higgs boson, missing from the particle spectrum. The Higgs field, with which the Higgs boson is directly related, plays a crucial role in the SM by permeating all space to "give" masses to every particle in the particle spectrum. Figure 1.2 summarizes the interactions among all fundamental particles, including the Higgs boson, described by the minimal SM. If the existence of the Higgs boson is proved experimentally, then the SM is complete. On the contrary, if the Higgs boson cannot be found, the SM then needs to be modified or replaced by other theories. Whether the Higgs boson can be found experimentally is thus a test of the kernel of the SM.

\subsubsection{The Higgs Mechanism for Boson Masses}

The Higgs mechanism is a process for gauge bosons to acquire non-zero masses through absorption of Goldstone bosons arising in the spontaneous symmetry break- 


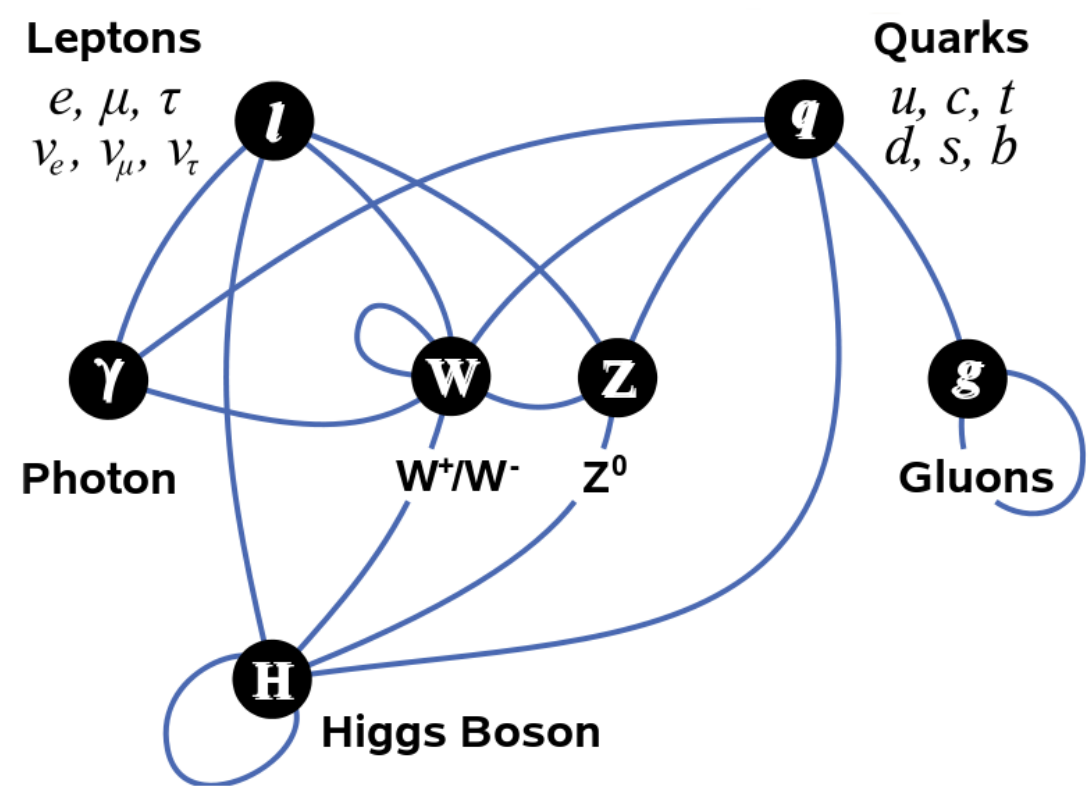

FiguRE 1.2: Summary of particle interactions described by the SM, assuming the Higgs boson exists.

ing [4]. Goldstone bosons are massless bosons that appear necessarily when the continuous symmetries in models are spontaneously broken, which happens when the states of lowest energy in the systems are degenerate. If the ground state of the system is not unique, the symmetry will be spontaneously broken when one particular ground state has been chosen. In the SM, the Higgs mechanism is used to break the electroweak $S U_{L}(2) \times U_{Y}(1)$ local gauge symmetry, giving rise to the masses of $W$ and $Z$ bosons, and the fermions.

The SM electroweak Lagrangian is given by [5]:

$$
\begin{aligned}
\mathcal{L}_{E W K}=\mathcal{L}_{0}+\mathcal{L}_{\phi}= & \left\{-\frac{1}{4} B_{\mu \nu} B^{\mu \nu}-\frac{1}{4} W_{\mu \nu} W^{\mu \nu}+\mathcal{L}_{\text {fermion }}\right\} \\
& +\left\{\left(D_{\mu} \phi\right)^{\dagger}\left(D^{\mu} \phi\right)-\left[-\mu^{2} \phi^{\dagger} \phi+\lambda\left(\phi^{\dagger} \phi\right)^{2}\right]\right\}
\end{aligned}
$$


with

$$
\begin{aligned}
B_{\mu \nu} & =\partial_{\mu} B_{\nu}-\partial_{\nu} B_{\mu} \\
W_{\mu \nu} & =\partial_{\mu} W_{\nu}-\partial_{\nu} W_{\mu}-g W_{\mu} \times W_{\nu} \\
D_{\mu} & =\partial_{\mu}+i g W_{\mu} \cdot T+i g^{\prime} B_{\mu} Y / 2
\end{aligned}
$$

where $\mathcal{L}_{0}$ is the zero-mass Lagrangian which incorporates the $S U_{L}(2) \times U_{Y}(1)$ symmetry of electroweak dynamics, $\mathcal{L}_{\phi}$ is the scalar field Lagrangian which is responsible for introducing couplings of the gauge fields to the Higgs field and generating masses for the $W^{ \pm}$and $Z$ bosons. The covariant derivative $D_{\mu}$ is chosen so that $\mathcal{L}_{0}$ is invariant under $S U_{L}(2) \times U_{Y}(1)$ gauge transformation. Under those transformations, the fermion field will transform as:

$$
\begin{aligned}
U_{Y}(1): & S U_{L}(2): \\
\psi_{L} \rightarrow e^{-i g^{\prime} \beta(x) Y / 2} \psi_{L} & \psi_{L} \rightarrow e^{-i g \alpha(x) \cdot T} \psi_{L} \\
\psi_{R} \rightarrow e^{-i g^{\prime} \beta(x) Y / 2} \psi_{R} & \psi_{R} \rightarrow \psi_{R}
\end{aligned}
$$

where $T$ relates to Pauli matrices $T=\frac{1}{2} \sigma, g^{\prime}$ and $g$ are coupling strengths for hypercharge vector potential $B_{\mu}$ (analogous to the EM vector potential $A_{\mu}$ ) and weak vector potential $W_{\mu}$, respectively. To make the fermion Lagrangian invariant under these gauge transformations, $W_{\mu}$ and $B_{\mu}$ need to transform as

$$
\begin{aligned}
U_{Y}(1) & : & S U_{L}(2): & \\
W_{\mu} & \rightarrow W_{\mu} & W_{\mu} & \rightarrow W_{\mu}+\partial_{\mu} \alpha(x)+g \alpha(x) \times W_{\mu} \\
B_{\mu} & \rightarrow B_{\mu}+\partial_{\mu} \beta(x) & B_{\mu} & \rightarrow B_{\mu}
\end{aligned}
$$

In the Glashow-Weinberg-Salam electroweak theory, the electromagnetic force and the weak force are not exactly unified but are "mixed" with each other. There three gauge bosons called $W_{\mu}^{1}, W_{\mu}^{2}$ and $W_{\mu}^{3}$ are associated with the weak force, while a single gauge boson $B_{\mu}$ is associated with the electromagnetic force. The $W^{ \pm}$, the 


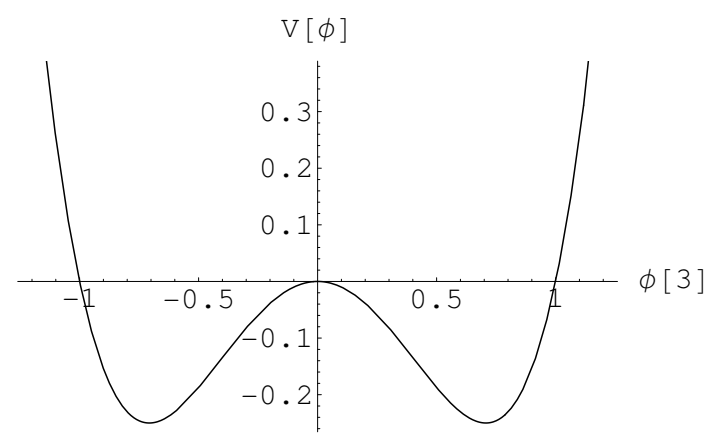

Figure 1.3: Scalar potential $\mathrm{V}(\phi)(\mu=\lambda=1$ case $) . \phi_{3}$ is the real part of the neutral component of $\phi$.

quantum of the charged weak force, is formed by the superpositions of $W_{\mu}^{1}$ and $W_{\mu}^{2}$ :

$$
W_{\mu}^{ \pm}=\left(W_{\mu}^{1} \mp i W_{\mu}^{2}\right) / \sqrt{2}
$$

A mixture of $W_{\mu}^{3}$ and $B_{\mu}$ via a rotation by the Weinberg weak mixing angle $\theta_{W}$ from $\left(W_{\mu}^{3}, B_{\mu}\right)$ gives observable vector potentials $\left(Z_{\mu}, A_{\mu}\right)$, as shown below

$$
\begin{aligned}
& Z_{\mu}=\cos \theta_{W} W_{\mu}^{3}-\sin \theta_{W} B_{\mu} \\
& A_{\mu}=\sin \theta_{W} W_{\mu}^{3}+\cos \theta_{W} B_{\mu} .
\end{aligned}
$$

The scalar potential component of $\mathcal{L}_{\phi}$ like the one shown in Figure 1.3 is the key construction of the Higgs mechanism:

$$
V(\phi)=-\mu^{2} \phi^{\dagger} \phi+\lambda\left(\phi^{\dagger} \phi\right)^{2}
$$

where $\mu^{2}$ and $\lambda$ are real positive numbers, $\phi=\left(\frac{\phi_{1}+i \phi_{2}}{\sqrt{2}}, \frac{\phi_{3}+i \phi_{4}}{\sqrt{2}}\right)$ is a complex scalar $S U(2)$ doublet with $\frac{\phi_{1}+i \phi_{2}}{\sqrt{2}}$ and $\frac{\phi_{3}+i \phi_{4}}{\sqrt{2}}$ representing the charged and neutral component of $\phi$, respectively. Once the non-zero minimum potential value where $\phi^{\dagger} \phi=$ $\left(\phi_{1}^{2}+\phi_{2}^{2}+\phi_{3}^{2}+\phi_{4}^{2}\right) / 2=\mu^{2} /(2 \lambda)$ is achieved, the electroweak symmetry is broken. To choose a direction in $S U(2)$ space and expand around the minimum, the appropriate choice is $\left\langle\phi_{1}\right\rangle=\left\langle\phi_{2}\right\rangle=0$ because the vacuum is neutral, and $\left\langle\phi_{4}\right\rangle=0$, 
$\left\langle\phi_{3}\right\rangle=\left(\mu^{2} / \lambda\right)^{1 / 2}$, with $\left(\mu^{2} / \lambda\right)^{1 / 2}$ labelled as the vacuum expectation value $v$. To explicitly recognize this vacuum reference value $v$, the $\phi_{3}$ scalar field can be written as $\phi_{3}=(v+H) / \sqrt{2}$ with $H$ called the Higgs field. Substitute the $\phi$ field into $\mathcal{L}_{\phi}$ and showing only the terms arising from $\phi_{3}$, we have [5] [6]:

$$
\begin{aligned}
& \mathcal{L}_{\phi}=\frac{1}{2}\left(\partial_{\mu} H\right)\left(\partial^{\mu} H\right)+\frac{1}{4} g^{2} W_{\mu}^{+} W^{\mu-}(v+H)^{2}+ \frac{1}{8} g_{Z}^{2} Z_{\mu} Z^{\mu}(v+H)^{2} \\
&-\left[-\frac{\mu^{2}}{2}+\frac{\lambda}{4}(v+H)^{2}\right](v+H)^{2} \\
&=\frac{1}{2}\left(\partial_{\mu} H\right)\left(\partial^{\mu} H\right)+\frac{1}{4} \mu^{2} v^{2}+\underbrace{\frac{1}{4} g^{2} v^{2} W_{\mu}^{+} W^{\mu-}}_{m_{W}^{2} W_{\mu}^{+} W^{\mu-}}+\underbrace{\frac{1}{8} g_{Z}^{2} v^{2} Z_{\mu} Z^{\mu}}_{\frac{1}{2} m_{Z}^{2} Z_{\mu} Z^{\mu}}-\underbrace{\mu^{2} H^{2}}_{\frac{1}{2} m_{H}^{2} H^{2}} \\
&+\underbrace{\frac{1}{2} g^{2} v W_{\mu}^{+} W^{\mu-} H}_{W-H-W}+\underbrace{\frac{1}{4} g_{Z}^{2} v Z_{\mu} Z^{\mu} H}_{Z-H-Z}-\underbrace{\lambda v H^{3}}_{H-H-H} \\
&+\underbrace{\frac{1}{4} g^{2} W_{\mu}^{+} W^{\mu-} H^{2}}_{W-W-H-H}+\underbrace{\frac{1}{8} g_{Z}^{2} Z_{\mu} Z^{\mu} H^{2}}_{Z-Z-H-H}-\underbrace{\frac{\lambda}{4} H^{4}}_{H-H-H-H} .
\end{aligned} .
$$

By inspecting the expansion coefficients, the boson masses can be identified as below:

$$
\left\{\begin{array}{l}
m_{W}=g v / 2 \\
m_{Z}=g_{Z} v / 2 \\
m_{A}=0
\end{array}\right.
$$

and

$$
m_{H}=\left(2 \mu^{2}\right)^{1 / 2}=\left(2 v^{2} \lambda\right)^{1 / 2}
$$

The coupling strengths $g$ and $g_{Z}$, or equivalently $g$ and $g^{\prime}$, are related by $\theta_{W}$ :

$$
e=g \sin \theta_{W}=g_{Z} \sin \theta_{W} \cos \theta_{W}=g^{\prime} \cos \theta_{W}
$$

with $e=\left(4 \pi \alpha_{E M\left(Q_{0}^{2}=m_{Z}^{2}\right.}\right)^{1 / 2}$. Since Fermi effective theory relates $g$ with $G_{F}$ constant [1] by

$$
g^{2}=\frac{8}{\sqrt{2}} G_{F} m_{W}^{2}
$$


we can thus relate the vacuum expectation value $v$ to precisely measured $G_{F}$ and get

$$
v=\frac{2 m_{W}}{g}=\left(\frac{1}{\sqrt{2} G_{F}}\right)^{1 / 2} \approx 246 \mathrm{GeV} .
$$

By using Eqn. (1.8) and Eqn. (1.9), we can get SM prediction for $W$ boson mass in terms of other parameters at tree level:

$$
m_{W}=\left(\frac{\pi \alpha_{E M}}{\sqrt{2} G_{F}}\right)^{1 / 2} \frac{1}{\left(1-m_{W}^{2} / m_{Z}^{2}\right)^{1 / 2}}
$$

where $\alpha_{E M}$ is the electromagnetic coupling evaluated at renormalization energy scale $Q=m_{Z}, G_{F}$ is the Fermi constant from the muon lifetime measurement.

When radiative loops are involved, the above equation can be generalized as:

$$
m_{W}=\left(\frac{\pi \alpha_{E M}}{\sqrt{2} G_{F}}\right)^{1 / 2} \frac{1}{\left(1-m_{W}^{2} / m_{Z}^{2}\right)^{1 / 2} \cdot \sqrt{1-\Delta r}}
$$

where $\Delta r$ parametrizes radiative corrections from light quarks, top loop and Higgs loop to the $W$ propagator and it can be decomposed into three main components:

$$
\Delta r=\Delta \alpha_{E M}+\Delta \rho\left(m_{t}^{2}\right)+\Delta \chi\left(\ln \left(m_{H}\right)\right)
$$

$\Delta \alpha_{E M}$ incorporates the contribution from the shift in the fine structure constant due to light fermions with $\Delta \alpha \propto \ln m_{f}, \Delta \rho$ represents the effect due to the quadratic dependence on top quark mass $m_{t}$, the third component $\Delta \chi$ arises due to the dependence on the logarithm of the Higgs boson mass $\ln m_{H}$. Figure 1.4 and Figure 1.5 show the Feymann diagrams corresponding to $\Delta \rho$ and $\Delta \chi$, respectively. When the radiative correction term $\Delta r=0$, Eqn. (1.12) gives the tree level result Eqn. (1.11).

\subsubsection{Mass Bounds of the Higgs Boson}

As shown in Eqn. (1.7) and Eqn. (1.10), the Higgs boson mass is given by $m_{H}=$ $\left(2 v^{2} \lambda\right)^{1 / 2}$ with $\lambda$ a dimensionless coupling constant and $v=246 \mathrm{GeV}$ to be the 


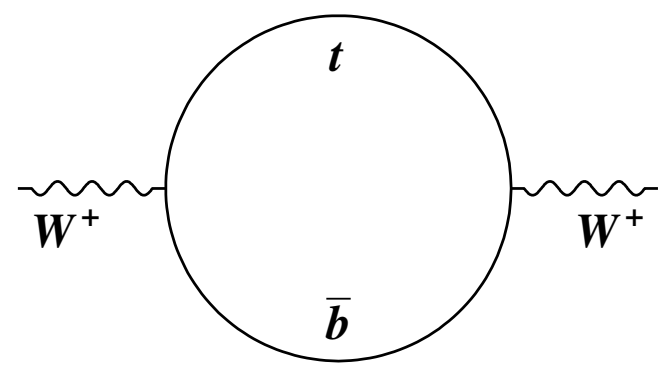

Figure 1.4: The one-loop contribution to the $W$ boson mass from top quark and bottom quark.
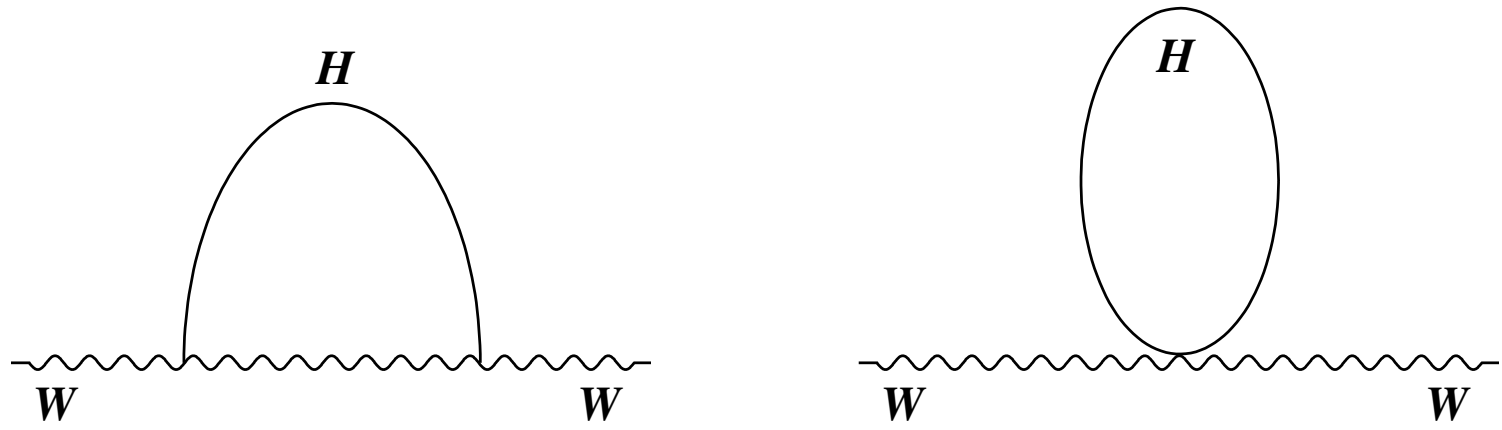

Figure 1.5: The one-loop contribution to the $W$ boson mass from the Higgs boson.

vacuum expectation value. The Higgs boson mass is arbitrary since $\lambda$ can be any positive value. Barger and Phillips [6] pointed out a lower limit on $\lambda$ could be set by considering effective self-interactions of gauge fields and the calculated lower bound of $m_{H}$ is $7 \mathrm{GeV}$. No rigorous theoretical upper limit on $m_{H}$ has been set, but to avoid unitarity violation, $m_{H}$ cannot be greater than $\sim 1 \mathrm{TeV}$, otherwise the width will be very broad and the Higgs boson can no longer be called as a "particle".

Since theories cannot predict the Higgs boson mass, it is thus necessary for experiments to search for the Higgs over all mass regions. Direct searches at LEP have excluded a light Higgs up to $m_{H}=114.4 \mathrm{GeV}$ at the $95 \%$ confidence level (CL) [7]. The latest results from Tevatron experiments CDF and D $\varnothing$, and Large Hadron Collider (LHC) experiments ATLAS and CMS, exclude a wide mass range of $m_{H}$ (see Figure 1.6), leaving an interesting mass region around $120 \mathrm{GeV} / \mathrm{c}^{2}$ yet to be 


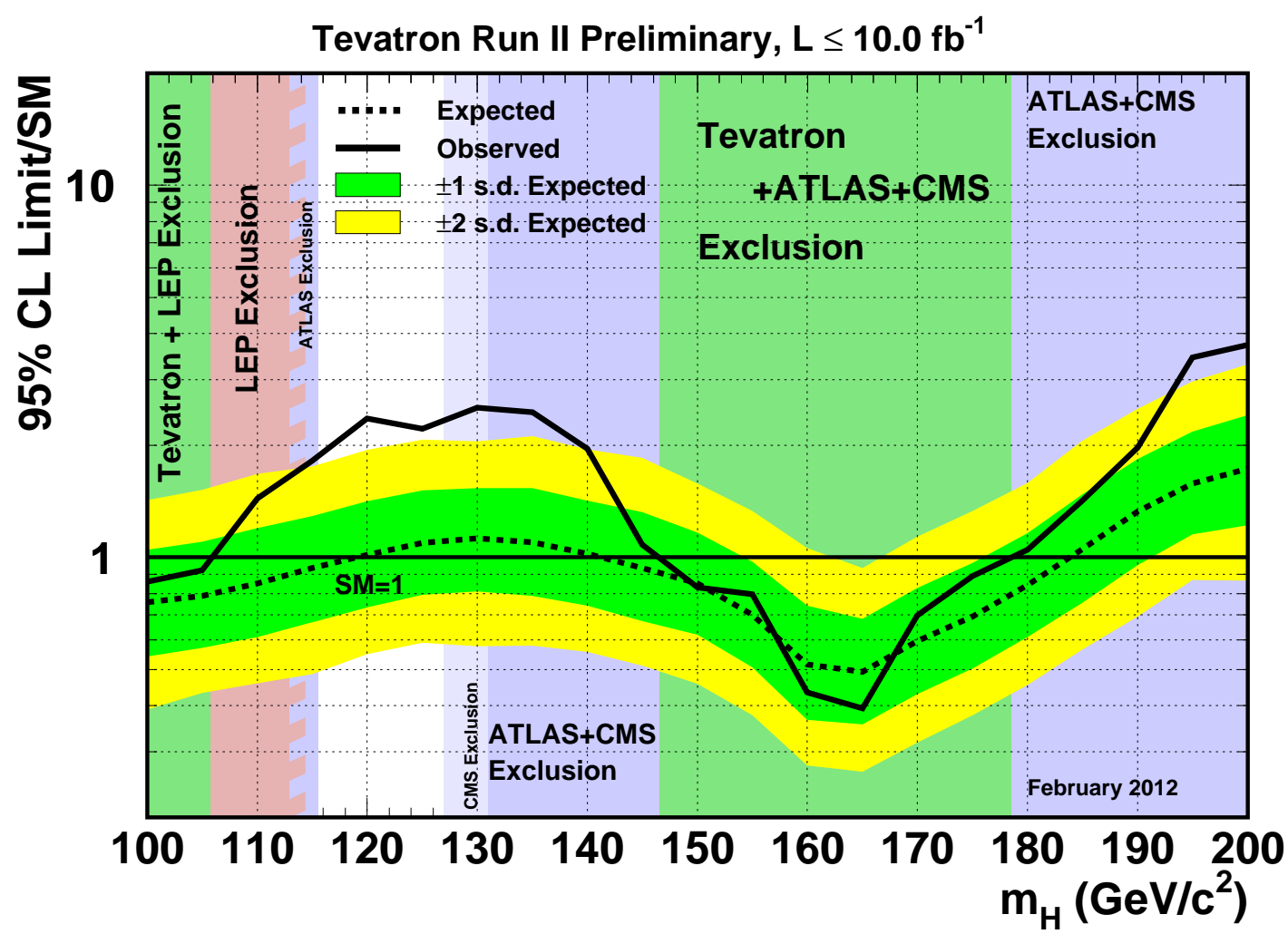

FiguRE 1.6: Higgs mass values excluded at $95 \%$ confidence level by indirect measurements and direct measurements from LEP, Tevatron and LHC experiments ATLAS and CMS. At the $95 \%$ confidence level (CL), LEP experiments exclude $m_{H}<114$ $\mathrm{GeV} / \mathrm{c}^{2}$; Tevatron experiments exclude $147 \mathrm{GeV} / \mathrm{c}^{2}<m_{H}<179 \mathrm{GeV} / \mathrm{c}^{2}$ and 100 $\mathrm{GeV} / \mathrm{c}^{2}<m_{H}<106 \mathrm{GeV} / \mathrm{c}^{2} ;$ CMS excludes $127 \mathrm{GeV} / \mathrm{c}^{2}<m_{H}<600 \mathrm{GeV} / \mathrm{c}^{2}$; ATLAS excludes $112.9 \mathrm{GeV} / \mathrm{c}^{2}<m_{H}<115.5 \mathrm{GeV} / \mathrm{c}^{2}, 131 \mathrm{GeV} / \mathrm{c}^{2}<m_{H}<238$ $\mathrm{GeV} / \mathrm{c}^{2}$ and $251 \mathrm{GeV} / \mathrm{c}^{2}<m_{H}<466 \mathrm{GeV} / \mathrm{c}^{2}$. [13]

further investigated. As of July 2011, the precision electroweak measurements, including the CDF $200 \mathrm{pb}^{-1} W$ mass measurement [8] [11] and the $\mathrm{D} \varnothing 1 \mathrm{fb}^{-1} W$ mass measurement [12], set an upper bound on the Higgs mass of $161 \mathrm{GeV}$ at the $95 \%$ confidence level [13]. Figure 1.7 shows $\Delta \chi^{2}$ curve derived from electroweak precision measurements as a function of $m_{H}$. Direct Higgs searches are being carried out at CDF and DØ experiments at Fermilab and is being further studied at the LHC at CERN. 


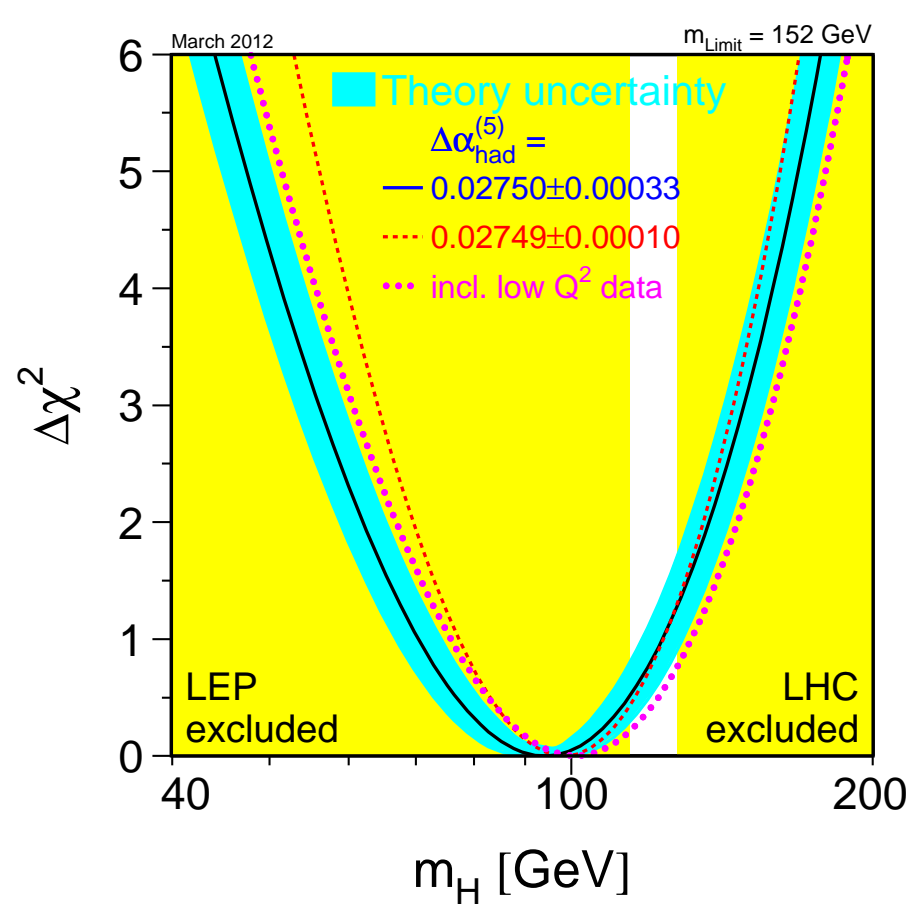

Figure 1.7: The $\Delta \chi^{2}$ as a function of $m_{H}$. This is derived from precision electroweak measurements, assuming the SM is the correct theory of nature. The minimum of the black curve is at $94 \mathrm{GeV}$ with an experimental uncertainty at $68 \% \mathrm{CL}$ of $+29 \mathrm{GeV}$ and $-24 \mathrm{GeV}$ which are derived from $\Delta \chi^{2}=1$ using the black curve. The blue band includes both the experimental and the theoretical uncertainties. The yellow region below $114 \mathrm{GeV}$ is excluded by LEP direct searches at 95\% CL. The yellow region above $127 \mathrm{GeV}$ is excluded by latest LHC searches at 95\% CL. [13]

\subsection{Motivation for $W$ Boson Mass Measurement}

The biggest motivation for precise $W$ mass $\left(m_{W}\right)$ measurement, together with the top quark mass $\left(m_{t}\right)$ measurement, is to constrain the undiscovered Higgs boson mass $\left(m_{H}\right)$ within the SM framework. According to Heisenberg uncertainty principle $(\Delta E \cdot \Delta t \geq \hbar / 2)$, energy conservation can be violated for a short period of time. Within that short period of time, the $W$ boson can fluctuate into other particles, including the Higgs boson, through radiative loops as shown in Figure 1.4 and Figure 
1.5. The $m_{W}$ is thus sensitive to $m_{H}$, allowing $m_{H}$ to be inferred from precise measurements of $m_{W}$ and $m_{t}$ (and other precision electroweak observables). We can see how these radiative corrections relate to $m_{W}$ with the following equation, which can be derived from Eqn. (1.12) by writing out $\Delta r$ terms [14]:

$$
\begin{aligned}
m_{W}= & 80.364+0.525\left[\left(\frac{m_{t}}{172}\right)^{2}-1\right]-0.0579 \ln \left(\frac{m_{H}}{100}\right)-0.008 \ln ^{2}\left(\frac{m_{H}}{100}\right) \\
& -0.5098\left(\frac{\Delta \alpha_{\text {had }}^{(5)}\left(m_{Z}\right)}{0.02761}-1\right)-0.085\left(\frac{\alpha_{s}\left(m_{Z}\right)}{0.118}-1\right)+\cdots .
\end{aligned}
$$

where $\Delta \alpha_{h a d}^{(5)}$ is the contribution to $\alpha_{E M}$ from the five quarks lighter than the top quark, and $\alpha_{s}\left(m_{Z}\right)$ is the strong coupling constant at the $Z$ mass energy scale. From Eqn. (1.14) we can see by fixing all other parameters except $m_{H}$ and $m_{W}$, higher values of $m_{W}$ suggests smaller values of $m_{H}$, and vice versa. Table 1.3 further shows changes in $m_{W}$ when other parameters are shifted. We can see uncertainties from $\Delta \alpha_{\text {had }}^{(5)}$ and $\alpha_{s}\left(m_{Z}\right)$ only affect $m_{W}$ by $\sim 2 \mathrm{MeV}$. Thus, relationship among $m_{W}, m_{t}$ and $m_{H}$ dominates Eqn. (1.14).

Table 1.3: Shifts in $m_{W}$ when varying $m_{H}$ by $+100 \mathrm{GeV}$ and other input parameters by $+1 \sigma$. The shifts are relative to the value $m_{W}=80.364 \mathrm{GeV}$, which is the result obtained when $m_{H}=100 \mathrm{GeV}$ and PDG central values of other parameters ( like $\alpha_{s}\left(m_{Z}\right)$ etc. $)$ are used.

\begin{tabular}{lr}
\hline \hline Parameter Shift & $\delta m_{W}(\mathrm{MeV})$ \\
\hline$\delta m_{H}=+100 \mathrm{GeV}$ & -43.4 \\
$\delta m_{t}=+1.1 \mathrm{GeV}[16]$ & +6.7 \\
$\delta \alpha_{s}\left(m_{Z}\right)=+0.0027[15]$ & -1.9 \\
$\delta\left(\Delta \alpha_{\text {had }}^{(5)}\right)=+0.00035[8]$ & -2.4 \\
\hline \hline
\end{tabular}

If we assume the central values $m_{W}=80.364 \mathrm{GeV}, m_{t}=172.0 \mathrm{GeV}$ and $m_{H}=$ $100 \mathrm{GeV}$, and neglect the small contributions from other parameters $\left(\Delta \alpha_{h a d}^{(5)}, \alpha_{s}\left(m_{Z}\right)\right.$ etc.), we can get the required precision of $m_{W}$ compared with the required precision of 
$m_{t}$ for the same constraining power on the precision of $m_{H}$. According to the numbers in Table 1.4 we find the $m_{W}$ and $m_{t}$ measurement uncertaintites, $\Delta m_{W}$ and $\Delta m_{t}$, need to satisfy the relationship $\Delta m_{W} \approx 0.006 \times \Delta m_{t}$ (see reference [15] for more discussion). Since the current Tevatron $m_{t}$ result has an uncertainty $\Delta m_{t}=0.9 \mathrm{GeV}$ [16], the relationship between $\Delta m_{W}$ and $\Delta m_{t}$ indicates that the equivalent $\Delta m_{W}$ needs to be about $5 \mathrm{MeV}$, which is however much smaller than the current worldaveraged measurement uncertainty of $23 \mathrm{MeV}$. Thus, to further constrain $m_{H}$, it is important to measure $m_{W}$ with higher precision. The $m_{W}, m_{t}$ and $m_{H}$ relationship is shown in Figure 1.8.

Table 1.4: Comparison of required precisions in $m_{t}$ and $m_{W}$ to constrain $m_{H}$ precision to $100 \mathrm{GeV}$.

\begin{tabular}{lrr}
\hline \hline Parameter Shift $(\mathrm{GeV})$ & $\Delta m_{t}(\mathrm{GeV})$ & $\Delta m_{W}(\mathrm{GeV})$ \\
\hline$\Delta m_{H}=+100$ & +7.362 & -0.043 \\
\hline \hline
\end{tabular}

A precise measurement of $m_{W}$, together with a precise measurement of $m_{t}$, can point out a mass window where direct searches for the Higgs boson should be focused if the SM is a self-consistent theory. If direct searches in that predicted mass window fail, then it indicates that the SM may just be an approximate description of our nature and new physics may be expected.

If the Higgs boson is found, the precise measurement of $m_{W}$ can be used to constrain the contributions arising from the extensions of the SM, which can be incorporated by introducing extra terms to Eqn. (1.14) due to non-SM particles. For example, contributions from supersymmetric particles are dominated by squark loops (see Figure 1.9), which can introduce several hundred MeV radiative corrections to $m_{W}$ [17]. With ultimate precision of the $W$ boson mass measurement, masses of SUSY particles can be inferred. 


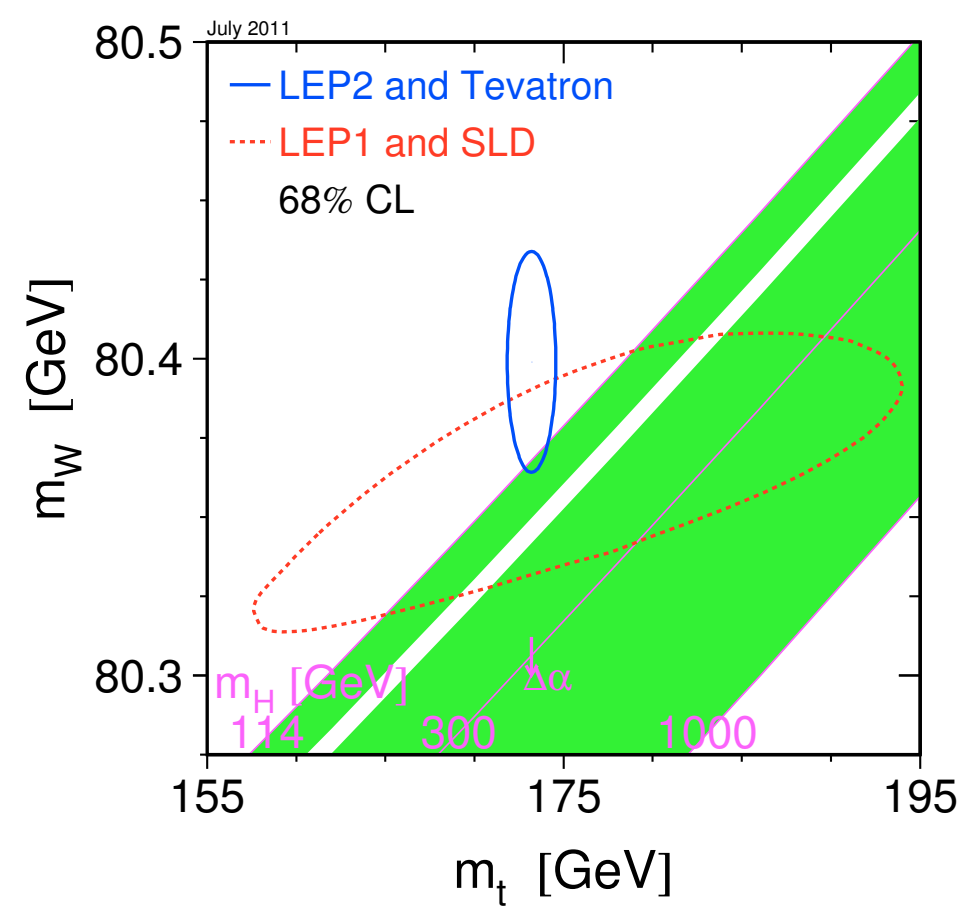

FiguRE 1.8: Direct $m_{W}$ and $m_{t}$ measurements, indirect constraints from $Z$-pole observables, and the SM theory curves relating $m_{W}$ and $m_{t}$ for fixed values of $m_{H}$ (as of July 2011) [13]. The white band region was excluded by 2011 Tevatron Higgs searches.
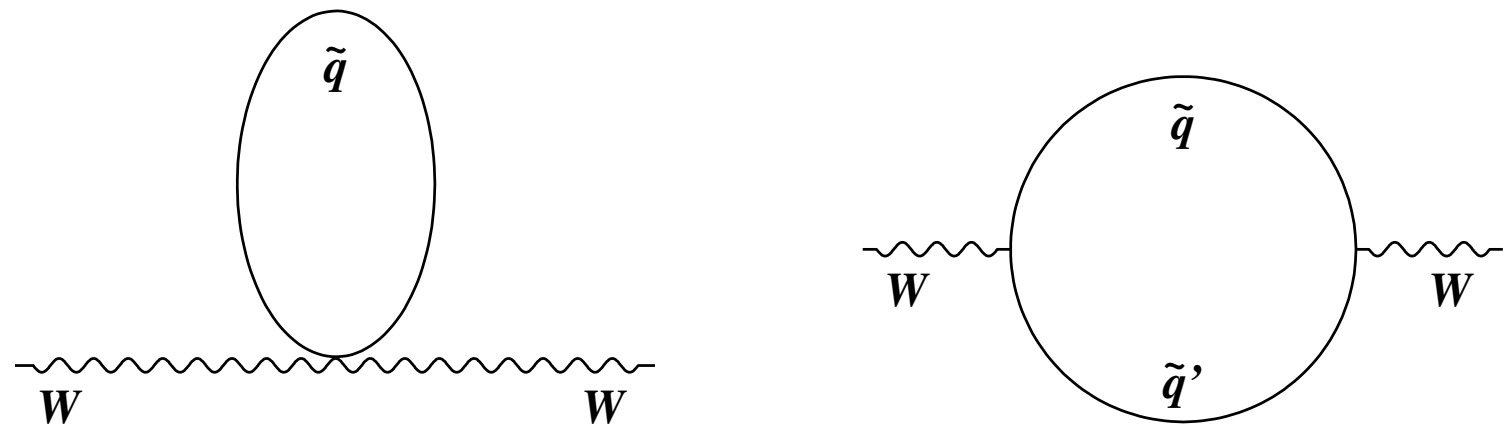

Figure 1.9: The one-loop contribution to the $W$ boson mass from squarks. 


\subsection{Past $W$ Boson Mass Measurements}

The existence of the massive weak force carriers, $W$ and $Z$ bosons, was predicted by the electroweak theory in 1967 and was confirmed later in 1983 by the UA1 and UA2 experiments at the CERN $S p \bar{p} S$ collider with a center-of-mass energy $\sqrt{s}=546 \mathrm{GeV}$. The mass of the $W$ boson was first measured to be $80 \pm 5 \mathrm{GeV}$ at that time [18] [19]. Serveral years later, the UA2 experiment published the first $m_{W}$ measurement with a precision less than $1 \mathrm{GeV}[20]$. Then four $e^{+} e^{-}$experiments at Large ElectronPositron collider (DELPHI, L3, OPAL and ALEPH) and two $p \bar{p}$ experiments at Fermilab Tevatron (DØ and CDF) pushed the world averaged measurement precision down to $23 \mathrm{MeV}$. Results of direct measurements of $W$ boson mass over last decade are shown in Table 1.5. The LEP experiments use $e^{+} e^{-} \rightarrow W^{+} W^{-}$process to measure $m_{W}$ and lead to a LEP-averaged $m_{W}$ with a precision of $33 \mathrm{MeV}$. The 2010 Tevatron average is better than LEP average mainly due to the contribution from the CDF and the DØ Run II measurements, with CDF using $200 \mathrm{pb}^{-1}$ data from both the electron channel $(W \rightarrow e \nu)$ and the muon channel $(W \rightarrow \mu \nu)$ while $\mathrm{D} \varnothing$ using $1 \mathrm{fb}^{-1}$ data on the electron channel only. The current world averaged $W$ boson mass before including this $2.2 \mathrm{fb}^{-1}$ measurement from CDF is $80399 \pm 23 \mathrm{MeV}$.

\subsection{Thesis Structure}

This thesis describes the $W$ boson mass measurement in $W \rightarrow \mu \nu$ channel with 2.2 $\mathrm{fb}^{-1}$ of CDF Run II data. Chapter 1 gives the introduction and the motivation. An overview of Fermilab accelerator complex and the CDF detector is presented in Chapter 2 and Chapter 3, respectively. A general picture of measurement strategy is shown in Chapter 4. Datasets and event selections used for this analysis are described in Chapter 5. The $W$ boson production and decay models are discussed in Chapter 6 followed by a description of the fast detector simulation in Chapter 7. In Chapter 
Table 1.5: $W$ mass measurements at LEP and Tevatron.

\begin{tabular}{lr}
\hline \hline Experiment & $m_{W}\left(\mathrm{MeV} / c^{2}\right)$ \\
\hline DELPHI [21] & $80336 \pm 67$ \\
L3 [22] & $80270 \pm 55$ \\
OPAL [23] & $80416 \pm 53$ \\
ALEPH [24] & $80440 \pm 51$ \\
\hline LEP Average [25] & $80376 \pm 33$ \\
\hline CDF Run I [26] & $80433 \pm 79$ \\
DØ Run I [27] & $80483 \pm 84$ \\
\hline Tevatron Run I Average [28] & $80454 \pm 59$ \\
\hline CDF Run II [29] & $80413 \pm 48$ \\
DØ Run II [30] & $80401 \pm 43$ \\
\hline Tevatron Run II Average (2010) [31] & $80420 \pm 31$ \\
\hline World Average (2010) [32] & $80399 \pm 23$ \\
\hline \hline
\end{tabular}

8, the momentum scale measurements using $J / \psi$ and $\Upsilon(1 S)$ samples are discussed in detail. Backgrounds and hadronic recoil model are shown in Chapter 9 and 10, respectively. The final $m_{W}$ results are presented in Chapter 11 . The whole thesis is summarized in Chapter 12, where the future perspective is also given.

The contribution of my work for the CDF $m_{W}$ analysis includes: Selection of $W \rightarrow \mu \nu, Z \rightarrow \mu \mu, J / \psi \rightarrow \mu \mu$ and $\Upsilon(1 S) \rightarrow \mu \mu$ samples; momentum scale measurements, combination and cross-checks using $J / \psi$ and $\Upsilon(1 S)$ samples; decorrelation of recoil response and resolution model parameters and evaluation of the corresponding systematic uncertainties; $W \rightarrow \mu \nu$ channel background measurements including $Z \rightarrow \mu \mu, W \rightarrow \tau \nu$, decay-in-flight and QCD jets; estimation of statistical correlations, as well as the statistical errors on correlation coefficients, among $m_{T}, p_{T}^{\mu}$ and $p_{T}^{\nu}$ fits for both $W \rightarrow \mu \nu$ and $W \rightarrow e \nu$ channels; combination of statistical uncertainties from $m_{T}, p_{T}^{\mu}$ and $p_{T}^{\nu}$ fits; estimation of the error on the combined statistical uncertainty due to the errors on statistical correlation coefficients; estimation of $Z / \gamma^{*}$ interference contributions to $Z \rightarrow \mu \mu$ mass fits; evaluation of the PDF uncertainties 
on $m_{W}$ in both $W \rightarrow \mu \nu$ and $W \rightarrow e \nu$ channels; combination of correlated $g_{2}$ and $\alpha_{s}$ systematic uncertainties; study of the effect on $m_{W}$ due to the QED second-photon radiation off the charged lepton by sampling WGRAD 2-d histogram of photon energy and angular distributions for the second time; $m_{W}$ cross-checks including fit window variations, $m_{W}^{\mu^{+}}$vs. $m_{W}^{\mu^{-}}, m_{W}\left(\phi_{\mu}>0\right)$ vs. $m_{W}\left(\phi_{\mu}<0\right)$ and run-dependence. 


\section{2}

\section{The Accelerator at Fermilab}

The Tevatron is a circular $p \bar{p}$ accelerator located at Fermilab in Batavia, Illinois. As a synchrotron, magnetic field and electric field, used to circulate and accelerate $p(\bar{p})$ respectively, are carefully synchronized with the travelling $p(\bar{p})$ beam momentum. The $p$ beam and $\bar{p}$ beam each carries $980 \mathrm{GeV}$ of energy thus provide a center of mass energy of $1.96 \mathrm{TeV}$ at two colliding points B0 and D0, where the Collider Detector at Fermilab (CDF) and the DØ detector are located. The Tevatron collider system consists of several production and acceleration related components: Proton Source, Main Injector, Antiproton Source, Recycler and Tevatron. The overview of the Tevatron Collider system is shown in Figure 2.1. A brief description of each component is given in the following sections.

\subsection{The Fermilab Accelerator Chain}

\subsubsection{The Proton Source}

The Proton Source consists of three accelerator systems: the Pre-accelerator, the Linac and the Booster.

The Pre-accelerator is the first accelerator of the Fermilab accelerator complex. 


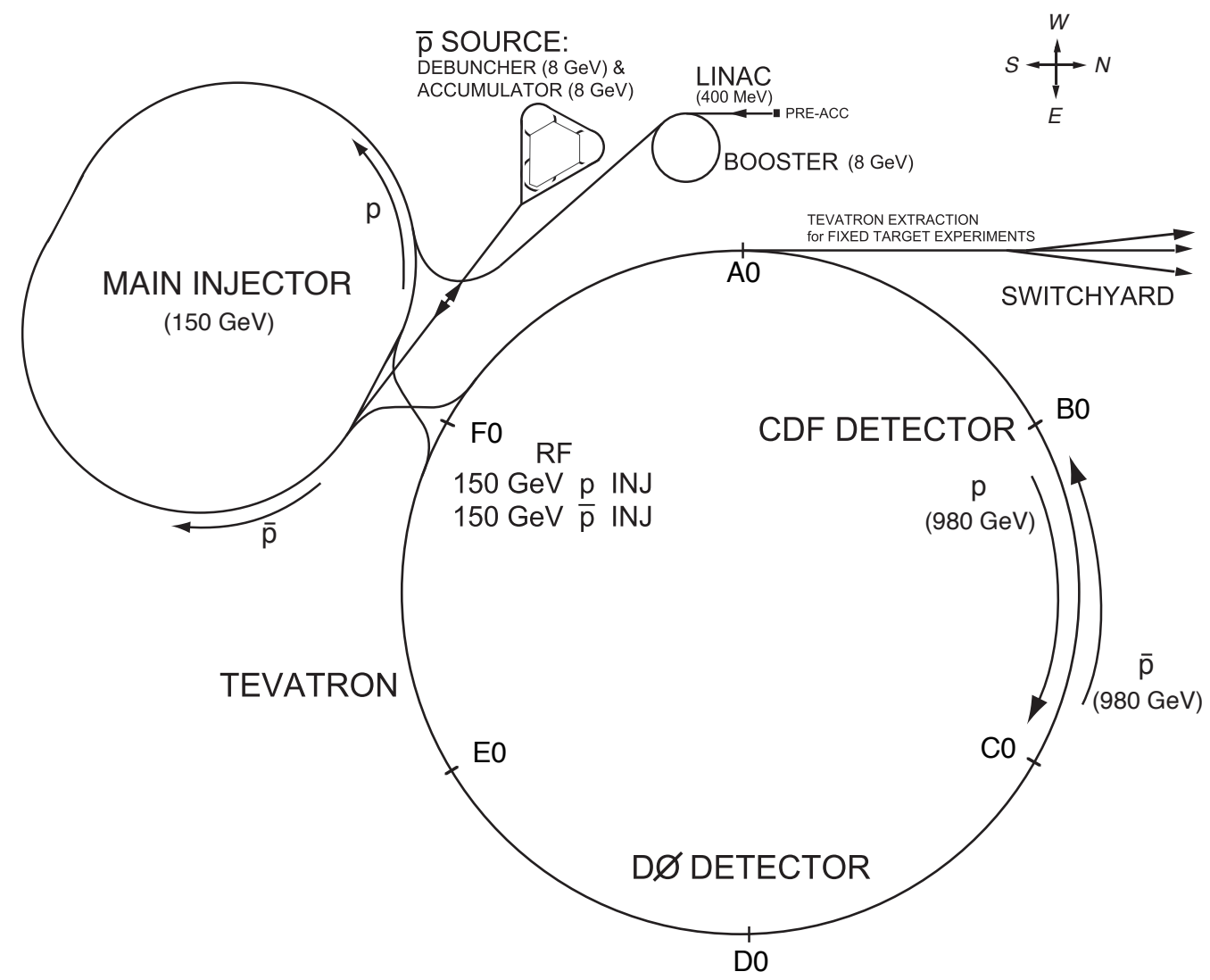

FiguRE 2.1: Overview of the Fermilab accelerator complex.

Here hydrogen gas is ionized to create negatively charged hydrogen $\left(H^{-}\right)$which is then accelerated to $750 \mathrm{KeV}$ through a column from the charged dome with potential $-750 \mathrm{kV}$ to the grounded wall.

The 155-meter Linear Accelerator (Linac) takes the $750 \mathrm{KeV}$ hydrogen ions and further accelerate them to $400 \mathrm{MeV}$. The accelerated ions are passed through a carbon foil to strip off the electrons from the ions such that only protons are left before entering the Booster.

Booster is a circular accelerator with a radius of 75 meters. Protons from Linac are accelerated in the Booster to an energy of $8 \mathrm{GeV}$. The Booster can accelerate the proton beam once every 66 milliseconds. 


\subsubsection{The Main Injector}

With a circumference about seven times that of the Booster, the Main Injector synchrotron is responsible to further accelerate the $8 \mathrm{GeV}$ protons from the Booster. Depending on the destination, the protons can be either accelerated to $120 \mathrm{GeV}$ or $150 \mathrm{GeV}$ in the Main Injector. For injection into the Tevatron, the protons are accelerated to an energy of $150 \mathrm{GeV}$. When sending beam to NuMI (Neutrinos at the Main Injector) or to the anti-proton source target station, the protons are accelerated to $120 \mathrm{GeV}$.

The Main Injector is also used to accelerate the $8 \mathrm{GeV}$ antiprotons from either the Accumulator or the Recycler (see Section 2.1.3) to $150 \mathrm{GeV}$ before injecting the antiprotons into the Tevatron.

\subsubsection{The Anti-Proton Source}

Producing antiprotons is not easy. The antiproton production rate at Fermilab is only $\sim 2 \times 10^{-5}$ per striking proton. Three major components - Target, Debuncher and Accumulator - are involved in the production of antiprotons.

- Target Station (see Figure 2.2):

A spray of secondary particles including antiprotons are produced by striking a beam of $120 \mathrm{GeV}$ protons from the Main Injector onto a Nickel alloy target. These secondary particles are further focused by a Lithium lens and separated by a dipole magnet. The produced antiprotons are then selected and sent to the Debuncher.

- The Debuncher:

Since the produced antiprotons have a large spread in momentum which is undesirable for high energy physics experiments, the Debuncher, an $8 \mathrm{GeV}$ synchrotron, is used to reduce the size and the momentum spread of the antipro- 


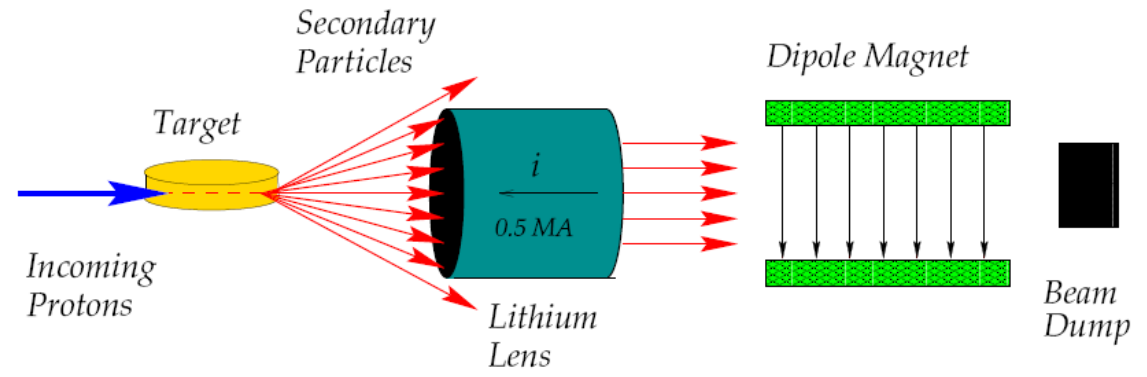

Figure 2.2: Antiproton production at the target station. The dipole magnets filter anti-protons and direct them to the Debuncher with the rest of the secondary particles sent to the beam dump.

tons to form an antiproton beam desirable for the accelerators downstream. The debunching process takes about 100 milliseconds.

- The Accumulator:

The Accumulator is a triangular-shaped synchrotron in the antiproton source system. It is used to accumulate $8 \mathrm{GeV}$ antiprotons by stacking antiproton pulses from the Debuncher. Antiprotons can be cooled and stored in the Accumulator for many hours.

\subsubsection{The Recycler}

The Recycler is an antiproton storage ring located in the Main Injector tunnel, directly above the Main Injector. The Recycler is used to reduce the logitudinal and transverse spread of the antiproton beam extracted from the Accumulator. This will provide a high intensity antiproton beam for the Tevatron.

\subsubsection{The Tevatron}

The Tevatron is a circular synchrotron with a circumference of approximately 4 miles. It is the largest accelerator of the Fermilab accelerator complex using more than 1,000 
superconducting magnets. Both protons and antiprotons are injected from the Main Injector into the Tevatron and are further accelerated within the Tevatron from 150 $\mathrm{GeV}$ to $980 \mathrm{GeV}$.

The beam delivered by the Tevatron consists of 3 trains, with each train containing of 12 bunches. Two subsequent trains are separated by $2.64 \mu$ s and two subsequent bunches within a train are separated by 396 ns. For a typical store, the number of protons per bunch is $2.7 \times 10^{11}$ and the number of antiprotons is $3.0 \times 10^{10}$. When in collision mode, the proton and antiproton beams are focussed by collimators into a cross-sectional area of $5 \times 10^{-5} \mathrm{~cm}^{2}$ at the collision points at which the CDF and the DØ detectors are centered. Table 2.1 summarizes the accelerators at Fermilab and the corresponding reachable energies.

Table 2.1: The Fermilab accelerator system.

\begin{tabular}{l|c|c|r}
\hline \hline Machine & Type & Particle & Energy \\
\hline \hline Preacc & Cockcroft-Walton & $H^{-}$ & $750 \mathrm{KeV}$ \\
\hline Linac & linear accelerator & $H^{-}$ & $400 \mathrm{MeV}$ \\
\hline Booster & synchrotron & $p$ & $8 \mathrm{GeV}$ \\
\hline Debuncher & synchrotron & $\bar{p}$ & $8 \mathrm{GeV}$ \\
\hline Accumulator & synchrotron & $\bar{p}$ & $8 \mathrm{GeV}$ \\
\hline Recycler & synchrotron & $\bar{p}$ & $8 \mathrm{GeV}$ \\
\hline Main Injector & synchrotron & $p$ and $\bar{p}$ & $150 \mathrm{GeV}$ \\
\hline Tevatron & synchrotron & $p$ and $\bar{p}$ & $980 \mathrm{GeV}$ \\
\hline \hline
\end{tabular}

\subsection{Tevatron Luminosity}

The instantaneous luminosity is an important parameter to quantify collider's performance. In the absence of a crossing angle or an offset in position, the expression below gives the definition of instantaneous luminosity at the interaction point [33]:

$$
L=\frac{n f N_{p} N_{\bar{p}}}{2 \pi\left(\sigma_{p}^{2}+\sigma_{\bar{p}}^{2}\right)} \cdot F\left(\frac{\sigma_{l}}{\beta^{*}}\right)
$$


where $n$ is the number of bunches in either $p$ or $\bar{p}$ beam (which are equal), $f$ is the frequency of collision, $N_{p}\left(N_{\bar{p}}\right)$ is the number of $p(\bar{p})$ per bunch, $\sigma_{p}\left(\sigma_{\bar{p}}\right)$ is the r.m.s. of the $p(\bar{p})$ beam size at the interaction point, $F$ is a form factor as a function of bunch length $\sigma_{l}$ and the amplitude function $\beta^{*}$ at the interaction point, where $\beta^{*}$ is a measure of beam width and is determined by the accelerator magnet configuration. Table 2.2 shows the designed Run II accelerator parameters [33]:

Table 2.2: Some designed accelerator parameters for Tevatron Run II.

\begin{tabular}{|c|c|}
\hline \multicolumn{2}{|c|}{ Accelerator parameters for Run II configuration } \\
\hline Parameter Name & Value \\
\hline Number of bunches $n$ & 36 \\
\hline Revolution frequency $f(\mathrm{MHz})$ & 1.7 \\
\hline Bunch spacing (ns) & 396 \\
\hline Number of $p$ per bunch $N_{p}$ & $2.7 \times 10^{11}$ \\
\hline Number of $\bar{p}$ per bunch $N_{\bar{p}}$ & $3.0 \times 10^{10}$ \\
\hline Bunch rms $\sigma_{l}(\mathrm{~m})$ & 0.37 \\
\hline$\beta^{*}(\mathrm{~cm})$ & 35 \\
\hline
\end{tabular}

The instantaneous luminosity $L$ decreases exponentially as a function of time from its peak value which is reached at the very beginning of a store. Figure 2.3 illustrates the instantaneous luminosity $L$ as a function of time. The Tevatron average store hours per week in FY 2010 is about 120. Through more efficient storage and more efficient transfer of antiprotons, the accelerator division at Fermilab improves the initial instantaneous luminosity over time, which is demonstrated by Figure 2.4. Figure 2.5 summarizes the integrated luminosity $\left(\mathcal{L}=\int L d t\right)$ delivered by Tevatron and acquired by the CDF detector since 2002. By the time Tevatron was shut down in September 2011, CDF detector has collected $\sim 12 \mathrm{fb}^{-1}$ data. More details on the Tevatron operation and performance can be found in [33] and [34]. 


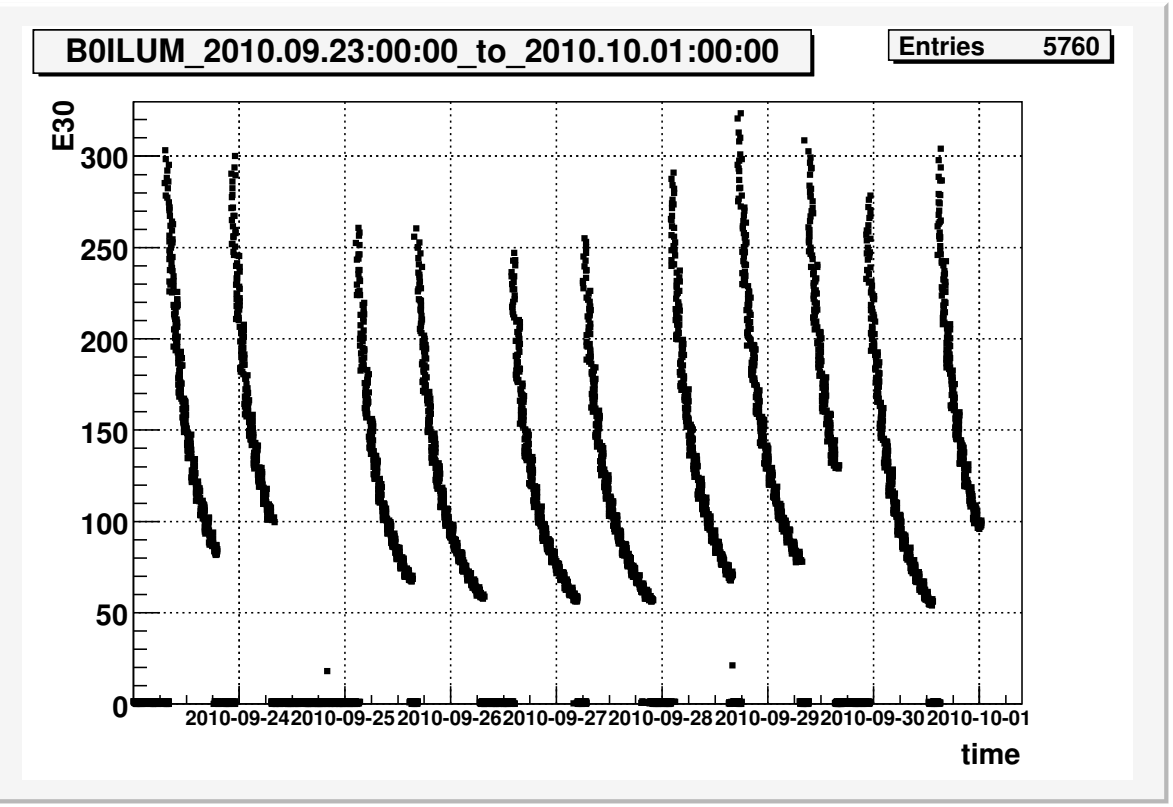

FIGURE 2.3: Instantaneous luminosity as a function of time over 11 stores in the week between Sept. 23, 2010 and Oct. 1, 2010.

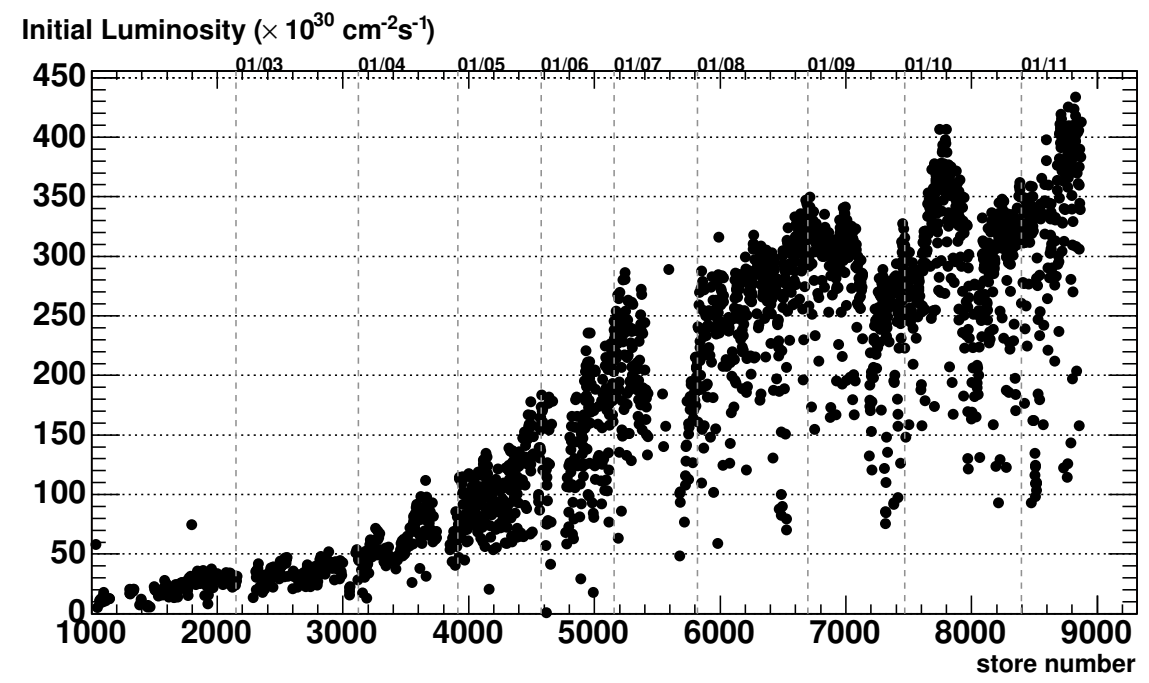

FIGURE 2.4: Initial instantaneous luminosity vs. store number. 


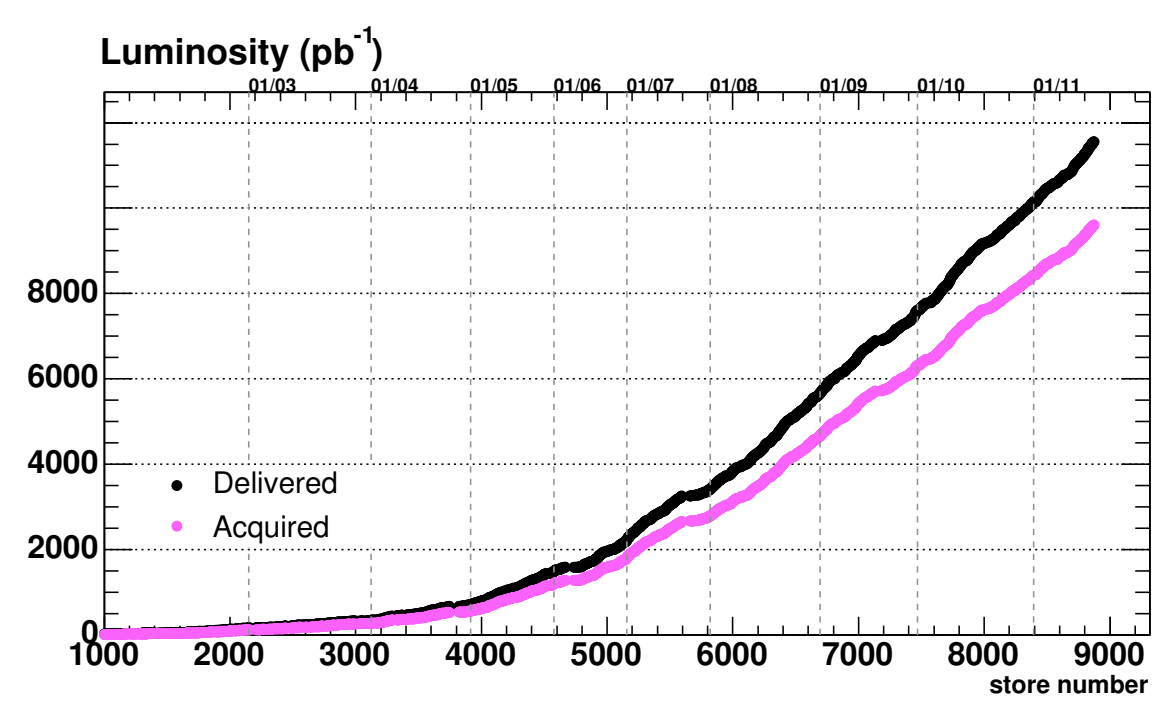

FiguRE 2.5: Integrated luminosity delivered to and acquired by the CDF detector as a function of time. 


\section{3}

\section{Detector}

The CDF detector is a multi-purpose spectrometer which is made up of the following sub-detectors from innermost to outmost regions: an inner silicon tracker to measure the production vertex of charged particles; a central outer tracker (COT) to precisely measure momenta of charged particles; a solenoid to provide uniform magnetic field inside COT along the beam direction; electromagnetic calorimeters to measure energy deposition from electron and photon showers; hadronic calorimeters to measure hadron energy and the muon detectors to detect muons. A schematic view of the CDF detector system is shown in Figure 3.1.

\subsection{The Coordinate System}

Both Cartesian and cylindrical coordinate systems are used in describing the CDF detector system and in this $W$ boson mass measurement. For Cartesian coordinate, the $+z$ axis is chosen to be in the direction of proton beam (east), the $+x$ axis is pointing outward from the Tevatron main ring and the $+y$ axis is pointing up (away from the earth). For cylindrical coordinate, the $+z$ axis is the same as the $+z$ axis in Cartesian coordinate system, $r$ is the radius from the $+z$ axis in the $x-y$ plane 


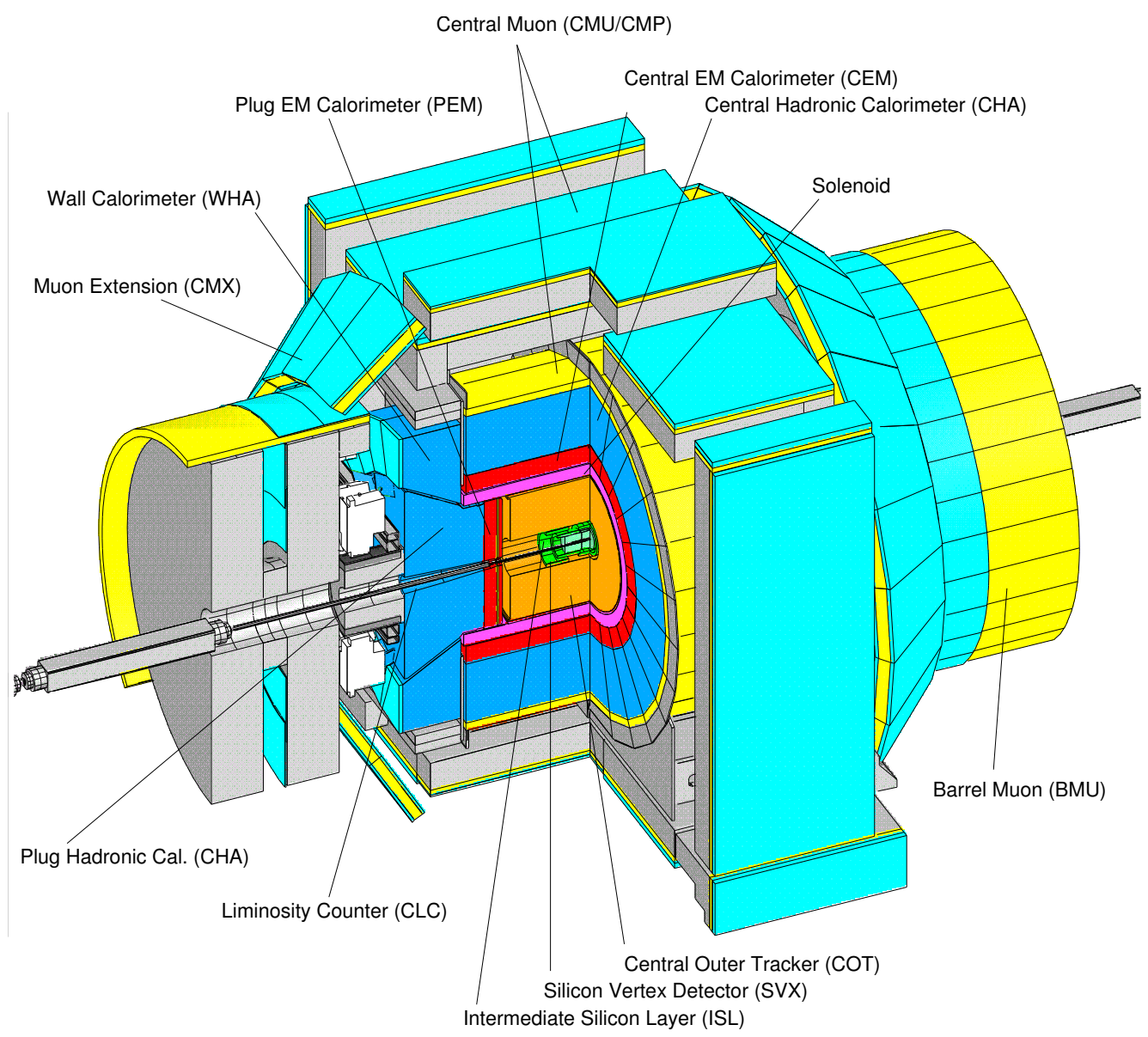

Figure 3.1: A schematic view of a section of the CDF detector system.

and $\phi$ is the azimuthal angle. In both Cartesian and cylindrical coordinate systems, the center of the detector is chosen as the origin. The polar angle $\theta$ is measured with respect to the $z$ axis. For massless particles, it is convenient to use pseudo-rapidity $\eta=-\ln [\tan (\theta / 2)]$ instead of $\theta$ since $\eta$ is additive under Lorentz boosts along the $z$-direction. In the CDF detector system, the central region covers $|\eta| \leq 1$ and the forward region covers $1.0 \leq|\eta| \leq 3.6$. Figure 3.2 shows the $\eta$ coverage of the CDF tracking system. 


\section{CDF Tracking Volume}

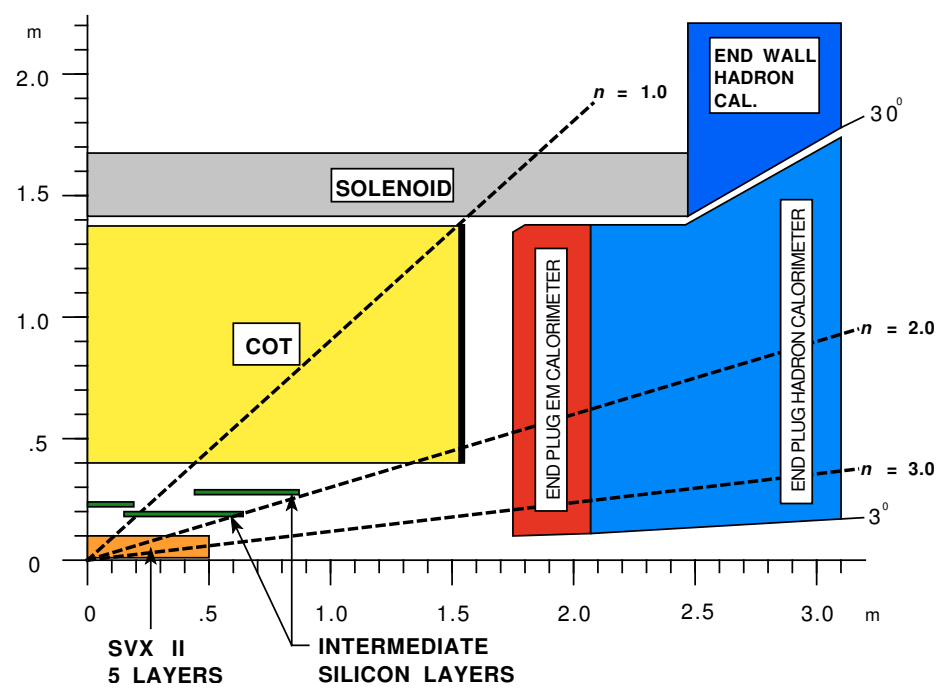

Figure 3.2: A schematic view of the $\eta$ coverage of the CDF tracking system.

\subsection{Silicon Tracker: Layer 00 + SVX II + ISL}

There are 3 sub-detectors in the silicon tracker system. From innermost to outermost, they are Layer 00 [36], SVX II [37] and ISL [38]. Figure 3.3 gives an end view of the silicon tracker. Layer 00 is a single layer of silicon sensors assembled on the beam pipe at radius of $1.3 \mathrm{~cm}$ with a thickness of $300 \mu \mathrm{m}$. It is the innermost silicon layer in the silicon tracker system and is designed to improve the impact parameter resolution significantly. The $90 \mathrm{~cm}$ long SVX II is segemented longitudinally into three cylindrical barrels. Each barrel supports five-layer silicon sensors covering the radii from $2.5 \mathrm{~cm}$ to $10.6 \mathrm{~cm}$. The intermediate silicon layer (ISL) has 3 layers at radii of $20.2 \mathrm{~cm}, 22 \mathrm{~cm}$ and $29.1 \mathrm{~cm}$ respectively, supplying additional detection between SVX II and COT. One of the 3 ISL layers $(r=22 \mathrm{~cm})$ is placed in the central region $(|\eta|<1.0)$ and the other two ISL layers are placed in the plug region $(1.0 \leq|\eta| \leq 2.0)$. SVX II and ISL can be used to provide silicon tracking and btagging over the region of $|\eta| \leq 2.0$. We do not use measurements from silicon tracker 
system in this $W$ mass analysis due to its limited angular coverage and considering the relatively small gain in resolution. However, the material effect on tracks due to silicon traker system is significant. For example, a typical track with $p_{T}=5 \mathrm{GeV} / \mathrm{c}$ from an $\Upsilon(1 S)$ decay will lose $\sim 9 \mathrm{MeV}$ due to silicon material [39]. The silicon tracker effect is incorporated in fast simulation by using a GEANT-4 [40] full-detector scanned map (SiliMap) which contains geometry information and radiation length at a given $(r, \phi, z)$.

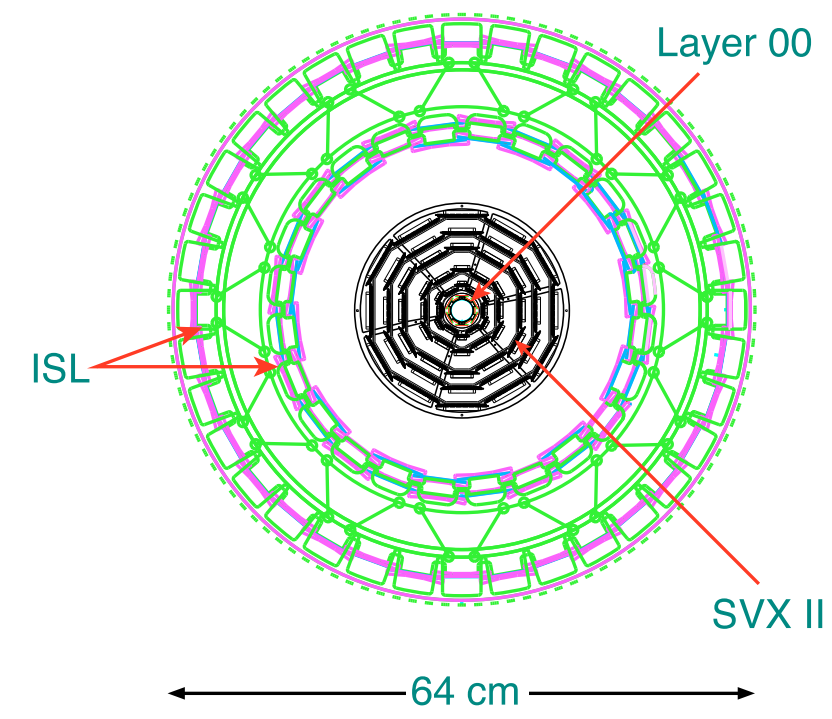

Figure 3.3: A view of the silicon detector along the beam direction $\hat{z}$.

\subsection{Central Outer Tracker: COT}

The Central Outer Tracker (COT) [35] is a $310 \mathrm{~cm}$ long open cell drift chamber between the Silicon detector and the time-of-flight system (TOF), covering radii between $40 \mathrm{~cm}$ and $137 \mathrm{~cm}$. All the three subdetectors (Silicon, COT and TOF) are within the coverage of the solenoid. The COT system is segmented into 8 superlayers (SLs), which alternate as stereo-axial-stereo-axial with the stereo angle of $2^{\circ}$. A basic 
drift cell from a superlayer consists of 12 sense wires, alternating with shaper wires radially. In each drift cell, 13 potential wires, which are used to supply close-touniform electric field, and 12 sense wires alternate with equal distances in a plane. Wire separation in the plane of the wires is $7.62 \mathrm{~mm}$. The cell geometry of SL2 (the second superlayer counting from innermost to outermost) is shown as an example in Figure 3.4. Both sense and potential wires are held and positioned at two endplates and there are altogether 30,240 sense wires and 32,760 potential wires in the entire COT. Figure 3.5 shows $1 / 6$ section of the COT endplate, where the enlargement shows in detail the geometry of field and sense slots. To prevent the relative deflection of sense wires within a cell under the influence of gravity, a support rod is used to connect sense wires at the COT center, which leads to no measurement of hits at a small region around $z=0$.

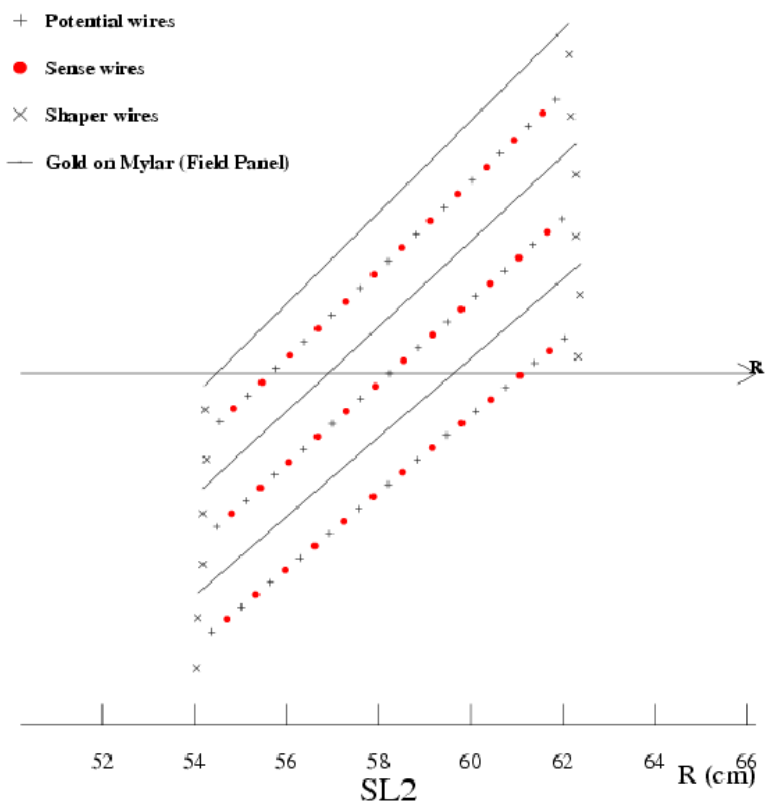

FigurE 3.4: Structure of SL2 and three COT cells are shown. Other superlayers are similar except for the taper. Each cell consists of 12 sense wires, 13 potential wires, 4 shaper wires and one Au-mylar cathode field panel on both sides of the sense/potential wire plane. 


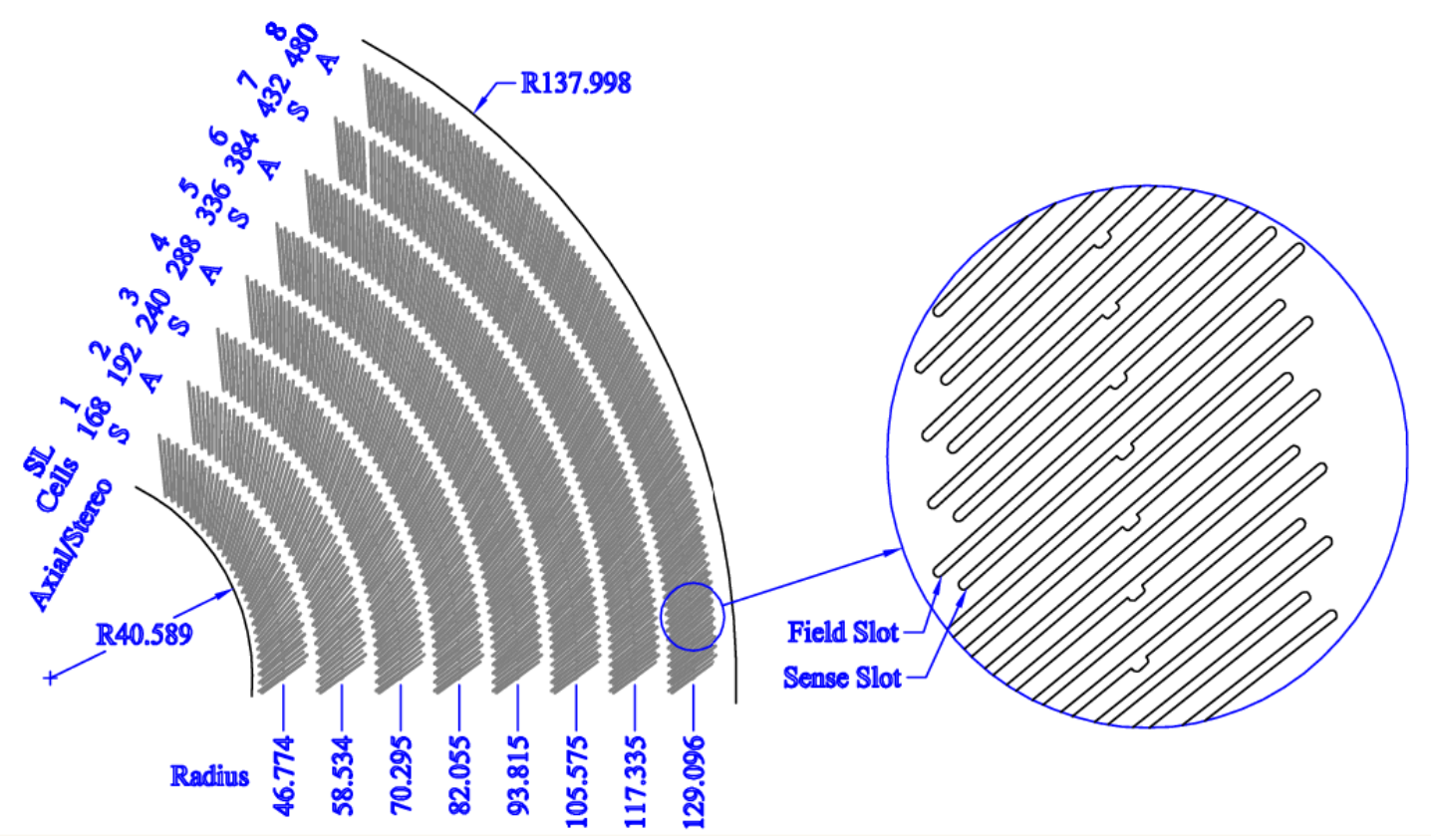

Figure 3.5: A view of $1 / 6$ section of the COT.

The COT is operated with an Argon-Ethane- $\mathrm{CF}_{4}$ gas mixture and the drift time is about 100 ns. Each drift cell is tilted counter-clockwisely by $35^{\circ}$ relative to the radial direction of the cell thus the drift direction of charged particles is azimuthal under the combined effect of electric and magnetic fields. Tracks from interaction point with $|\eta|<1.0$ can pass through all 8 COT superlayers at most; tracks with $1.0<|\eta|<1.3$ can pass through 4 to 8 superlayers.

Table 3.1: Some basic parameters of the COT.

\begin{tabular}{lcccccccc}
\hline \hline Mechanical parameters & SL1 & SL2 & SL3 & SL4 & SL5 & SL6 & SL7 & SL8 \\
\hline Number of cells & 168 & 192 & 240 & 288 & 336 & 384 & 432 & 480 \\
Number of sense wires per cell & 12 & 12 & 12 & 12 & 12 & 12 & 12 & 12 \\
Stereo angle $\left(^{\circ}\right)$ & +2 & 0 & -2 & 0 & +2 & 0 & -2 & 0 \\
Radius at SL center $(\mathrm{cm})$ & 47 & 59 & 70 & 82 & 94 & 106 & 117 & 129 \\
\hline \hline
\end{tabular}




\subsection{Calorimeter Systems}

The scintillator-based CDF calorimeter systems [41] are tower segmented and are located outside the solenoid, covering $|\eta|<3.6$ region. The calorimeter systems can be divided into two regions according to the $\eta$ coverage: the central region with $|\eta| \lesssim 1.1$ and the plug region with $|\eta| \gtrsim 1.1$. The central region consists of the Central Electromagnetic calorimeter (CEM) and the Central Hadronic calorimeter (CHA), which is located outside of the CEM. The plug region consists of the Wall Hadronic calorimeter (WHA), the Plug Electromagnetic calorimeter (PEM) and the Plug Hadronic calorimeter (PHA). Though WHA has components both in the central region and the plug region, it is traditionally viewed as a part of the plug. Each calorimeter tower covers $0.1 \times 15^{\circ}$ in $\eta \times \phi$ space and consists of alternating layers of material and scintillators, with a lead-scintillator E-M section in the inner region followed by a steel-scintillator hadron section in the outer region. The structure of a typical CEM wedge is shown in Figure 3.6. Table 3.2 summarizes some basic parameters of the calorimeter systems.

Table 3.2: Some basic parameters of the CDF calorimeter systems.

\begin{tabular}{lccccc}
\hline \hline & \multicolumn{3}{c}{ Central } & & Plug \\
& CEM & CHA & WHA & PEM & PHA \\
\hline Parameters & $<1.1$ & $<0.9$ & $(0.8,1.2)$ & $(1.1,3.6)$ & $(1.2,3.6)$ \\
Number of layers & 31 & 32 & 15 & 23 & 23 \\
Passive material & lead & iron & iron & lead & iron \\
Passive material thickness $(\mathrm{cm})$ & 0.32 & 2.5 & 5.0 & 0.45 & 2.5 \\
Scintilator thickness $(\mathrm{cm})$ & 0.5 & 1.0 & 1.0 & 0.4 & 1.0 \\
Radiation length $\left(X_{0}\right)$ & 18.0 & - & - & 21.0 & - \\
Nuclear interaction length $\left(\lambda_{0}\right)$ & - & 4.5 & 4.5 & - & 7.0 \\
\hline \hline
\end{tabular}

The calorimeter system plays a key role in measuring electron and photon energies, jet energies and net transverse energy. It can also be used to match tracks ex- 


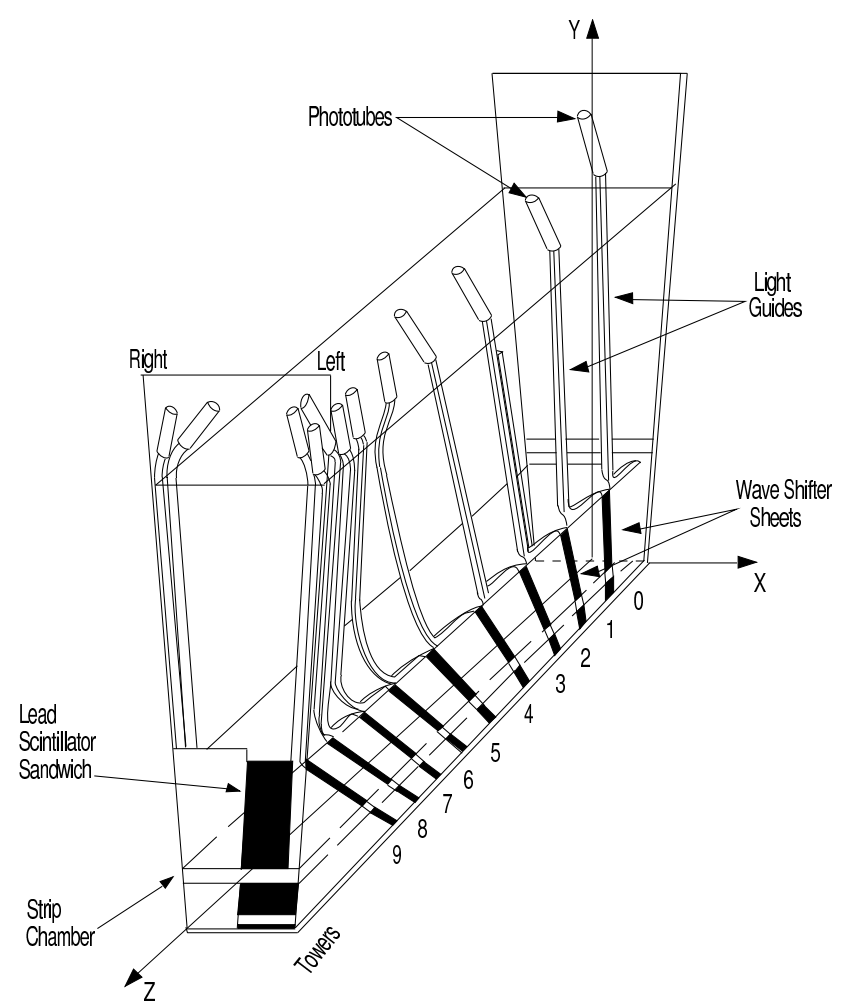

Figure 3.6: The structure of a typical CEM wedge.

trapolated from COT with tower and shower positions. As particles passing through the calorimeter, they interact with materials and produce secondary showers with energy deposition. Light is collected by scintilators and read out by wavelength shifting (WLS) fibers, then carried out to the photomultiplier tubes (PMT) by the connecting clear fibers. In PMTs, light signal is converted into electrical signal according to its intensity, thus making the strength of electrical signal propotional to the energy deposited in the calorimter.

Since we measure the $W$ boson mass using leptonic decay channels, neutrinos in final products will escape from our detector without being detected. However, we can still infer the missing energy of a neutrino $\left(\mathbb{E}_{T}\right)$ by taking the vectorial sum of 
transverse energies over all towers:

$$
E_{T}=\left|\overrightarrow{E_{T}}\right|=\left|-\sum_{i} \vec{E}_{T}^{i}\right|=\left|-\sum_{i} E_{T}^{i} \hat{n}_{i}\right|
$$

where $E_{T}^{i}=E^{i} \sin \theta$ with $E^{i}$ the magnitude of energy deposited in the $i$ th calorimeter tower and $\theta$ to be the polar angle with respect to $z$ axis; $\hat{n}_{i}$ is a unit vector pointing away from the $W$ boson production point to the $i$ th tower in the $r-\phi$ plane. To measure neutrino transverse missing energy precisely, we need a well-calibrated calorimeter with good energy resolution.

\subsection{Muon Systems}

The three relevant muon detectors for the $W$ mass measurement in the $|\eta|<1.0$ region are Central Muon Detector (CMU), Central Muon Upgrade (CMP) and Central Muon Extension (CMX), with CMU and CMP covering $|\eta|<0.6$ region while CMX covering $0.6<|\eta|<1.0[42]$.

The CMU detector, locating outside of the Central Hadronic Calorimeter (CHA) at $r=347 \mathrm{~cm}$, consists of 144 modules (72 modules in $z>0$ region and 72 modules in $z<0$ region) with 16 rectangular proportional drift chambers (cells) per module. It is segmented into 24 wedges azimuthally, with each $15^{\circ}$ wedge containing 3 modules. A schematic view of a typical CMU wedge is shown in Figure 3.7. The 16 cells in a module are arranged into four layers in the radial direction, as shown in the left plot of Figure 3.8. Each proportional drift cell has a size of $6.35 \mathrm{~cm} \times 2.68 \mathrm{~cm} \times 226 \mathrm{~cm}$ with a $50 \mu m$ thick steel wire in the cell center.

The CMP detector is located behind an additional $60 \mathrm{~cm}$ of steel in the region $|\eta|<0.6$. The CMP drift chambers are similar to the CMU drift chambers, with the exception that the CMP drift chambers are wider and alternate half-cell staggering. A schematic view of CMP cells is shown in the right plot of Figure 3.8. Since the 


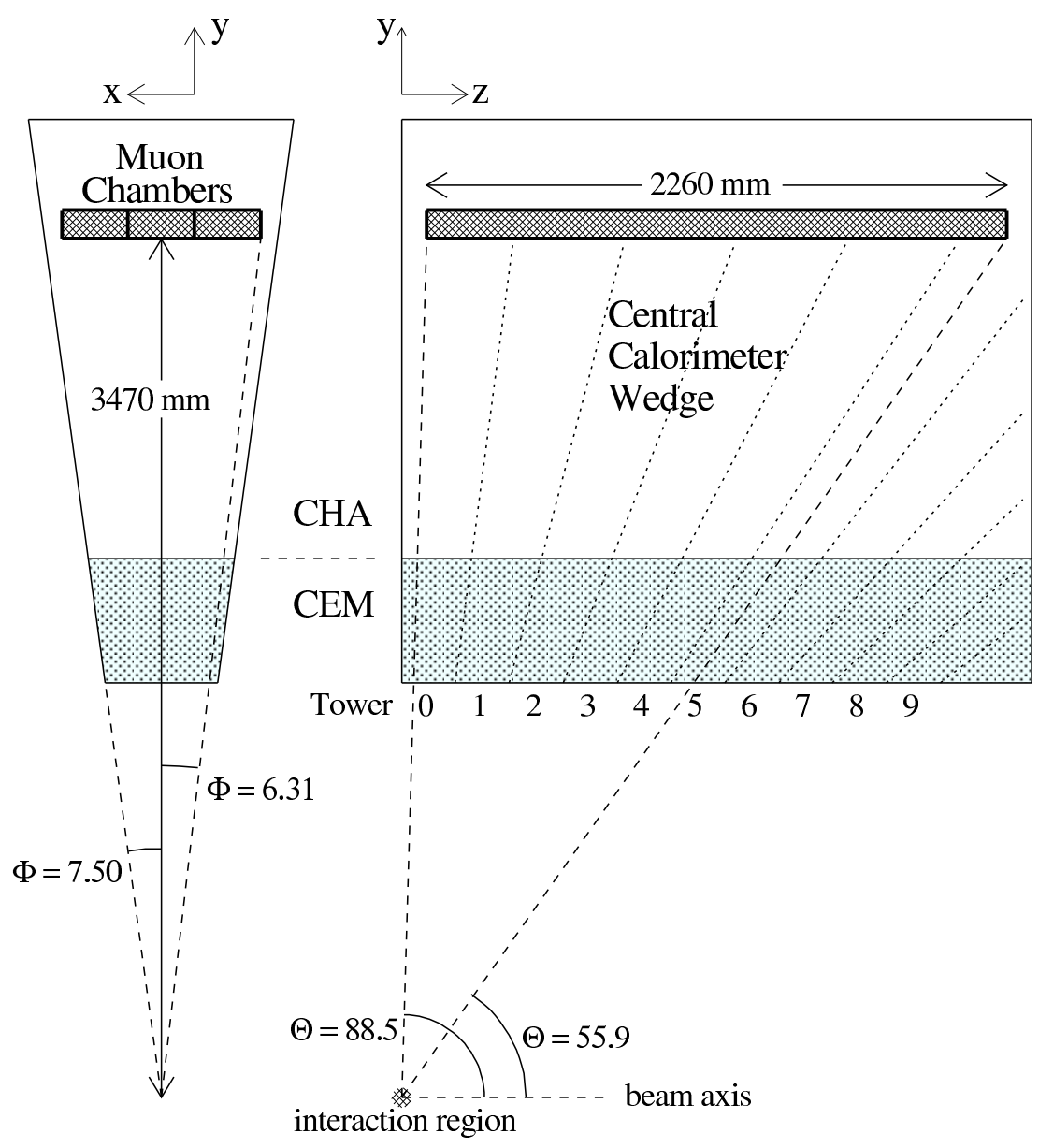

Figure 3.7: Schematic view of typical CMU wedge.
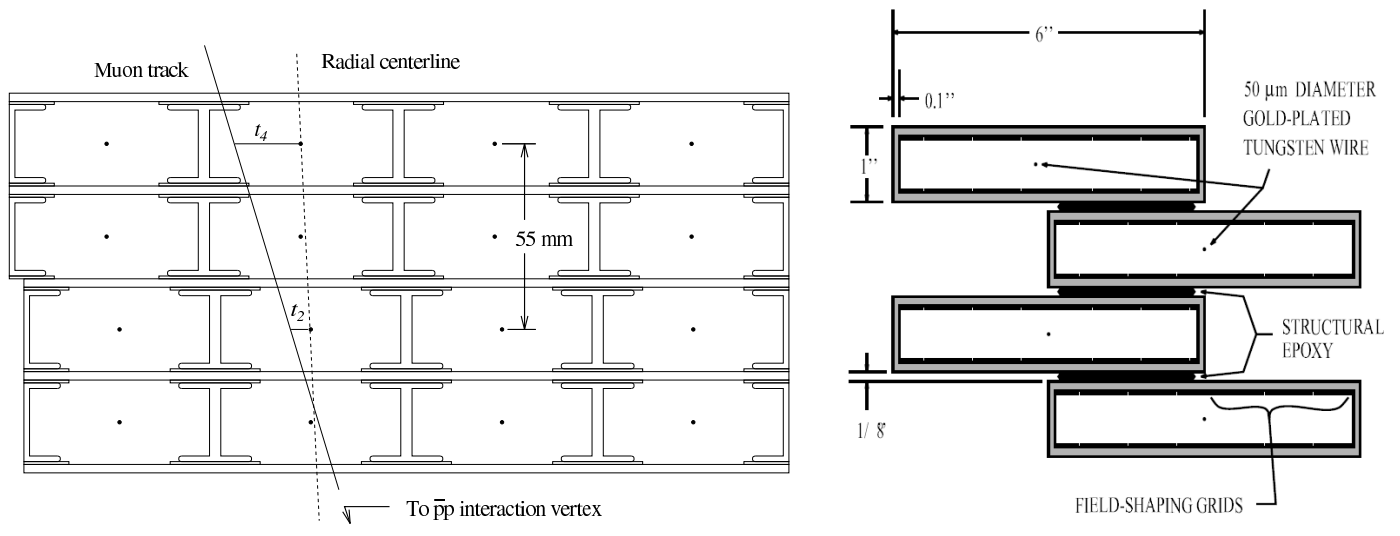

Figure 3.8: A view of CMU module (left) and CMP module (right) in $r-\phi$ plane. 
CMP chambers are of fixed length in $z$ and form a square box around the CMU, the $\eta$ coverage thus varies with azimuthal angle as shown in the Figure 3.9. To improve the muon identification, the CMP information is often used in conjunction with the CMU information.

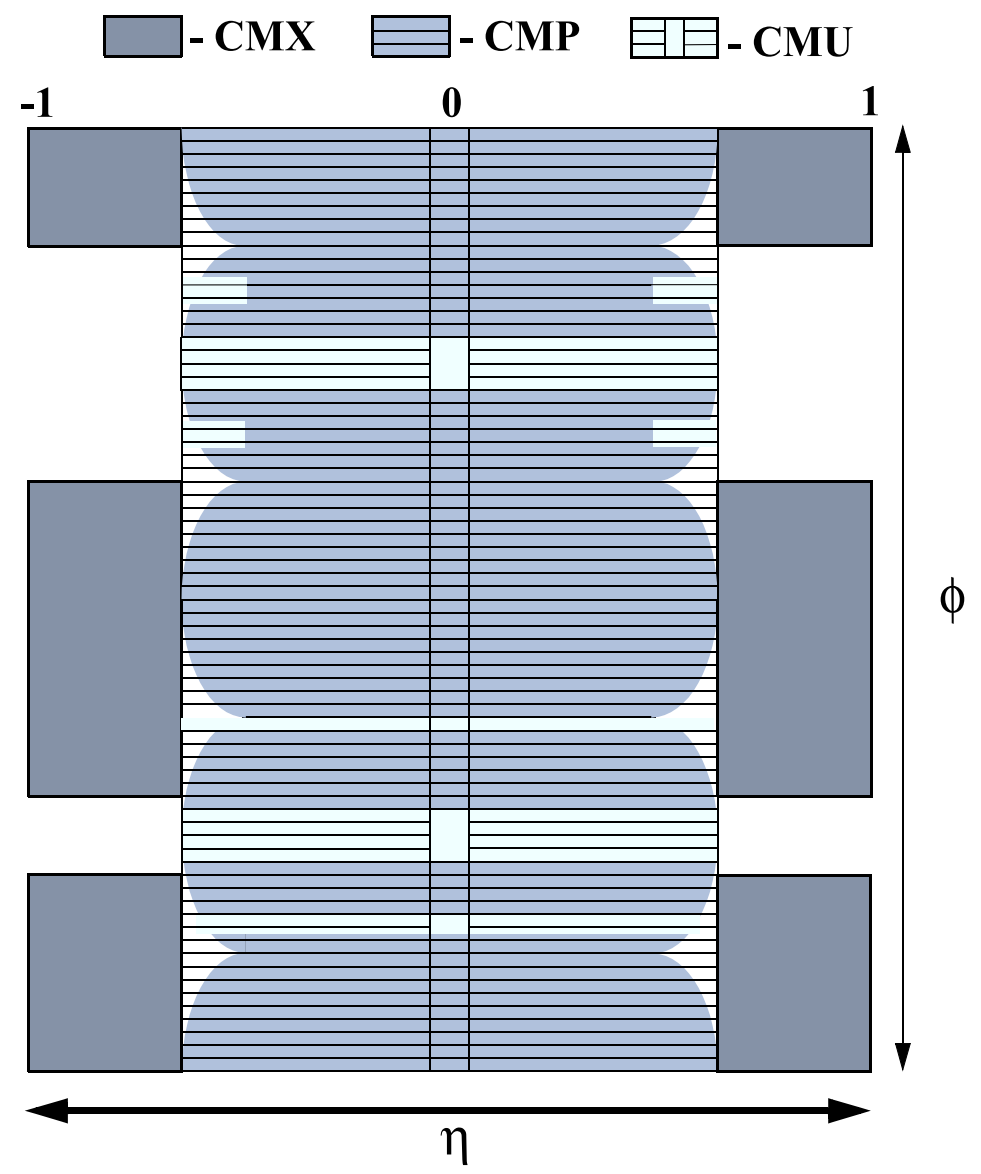

Figure 3.9: Coverage of CMU, CMP and CMX in $\phi-\eta$ plane.

The CMX extends the $|\eta|$ coverage to 1.0 to provide muon identification in the plug region. It consists of eight concentric layers which are arranged into pairs to form four layers. The segments are staggered with each other to provide $z$ measurements. The scintillation counters of the CMX, called CSX, are mounted outside of the CMX system. In our analysis, a fired segment in the muon system is often referred to as a muon "stub", which is further matched with the corresponding COT track 
information for muon identification.

\subsection{Cherenkov Luminosity Counter}

At CDF, the Cherenkov Luminosity Counter (CLC) system is responsible for measuring the luminosity. There are 48 isobutane-filled Cherenkov counters located close to the beamline at the two ends of the CDF detector, with $\eta$ coverage $3.6<|\eta|<4.6$. The CLC system accounts the number of particles emerging from the $p \bar{p}$ collisions at small angles to measure $n_{\text {in }}$ - the number of inelastic $p \bar{p}$ scattering per bunch crossing. Then the instantaneous luminosity $L$ is calculated by using

$$
L=\frac{n_{\text {in }} f}{\sigma_{\text {in }}}
$$

where $f$ is the frequency of collision $(f=1.7 \mathrm{MHz})$ and $\sigma_{\text {in }}$ is the measured cross section for inelastic $p \bar{p}$ collision $\left(\sigma_{\text {in }} \approx 60.7 \pm 2.4 \mathrm{mb}\right.$ at $\left.\sqrt{s}=1.96 \mathrm{TeV}[43]\right)$. Events collected by CLC system are known as "minimum bias" events, which are used in the recoil model study.

\subsection{Data Acquisition System}

The goal of trigger and DAQ system is to maximize data for desired process to storage for analysis with minimal cost. Since bunches circulate in three trains of 12 bunches with each bunch spaced $396 \mathrm{~ns}$, the average crossing rate at the Tevatron is 1.7 $\mathrm{MHz}^{1}$, which is too much for DAQ to handle. Since the majority of the collisions do not contain physics of interest, a three trigger system is used to reduce the rate down to $\sim 75 \mathrm{~Hz}$. This corresponds to an event reduction rate of $\sim 1: 25000$. Figure 3.10 shows the CDF data flow in the three level trigger architecture known as Level 1, Level 2 and Level 3. The first two levels are controlled by the Trigger Supervisor,

${ }^{1} \mathrm{f}=\#$ of bunches $\times$ revolution frequency $=\mathrm{n} \cdot \frac{c}{s}=36 \times 3 \cdot 10^{8} \mathrm{~m} / \mathrm{s} / 6.3 \cdot 10^{3} \mathrm{~m} \approx 1.7 \mathrm{MHz}$, where $c$ is speed of light, $s$ is the circumference of the Tevatron. 
which coordinates signals from global clock, bunch crossing and different parts of the trigger and DAQ system. Once an event passes through the Level 3, it is sent to the final component of the DAQ system - Consumer Server/Logger (CSL) - for storage first on disk than later on tape.

\section{Dataflow of CDF "Deadtimeless" Trigger and DAQ}

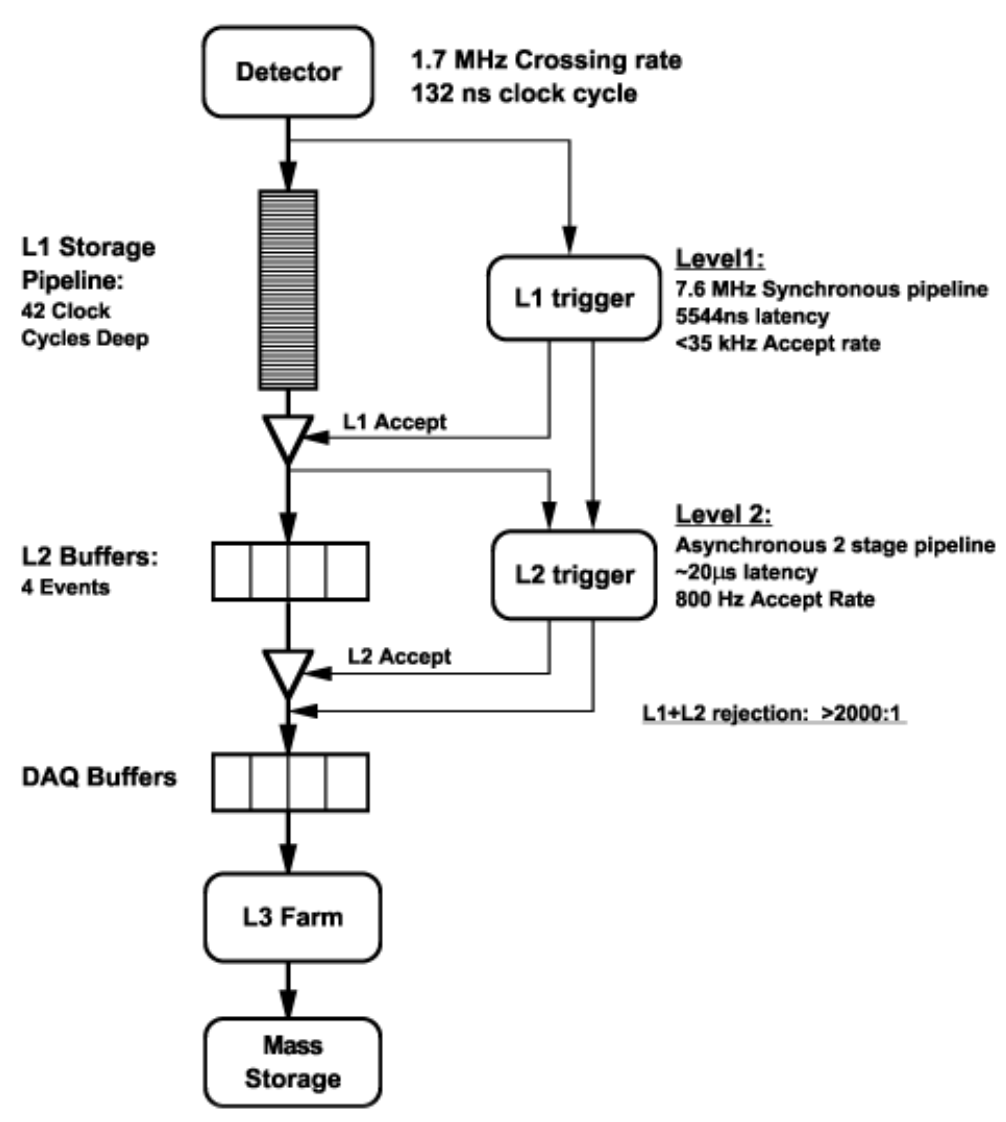

Figure 3.10: Diagram of the CDF dataflow [44].

The Level 1 trigger (L1) is the first decision and filtering level in the trigger system using purely hardward-based information from tracker, calorimeter and muon detector to decide whether an event is accepted or rejected. At this level, collision 


\section{RUN II TRIGGER SYSTEM}

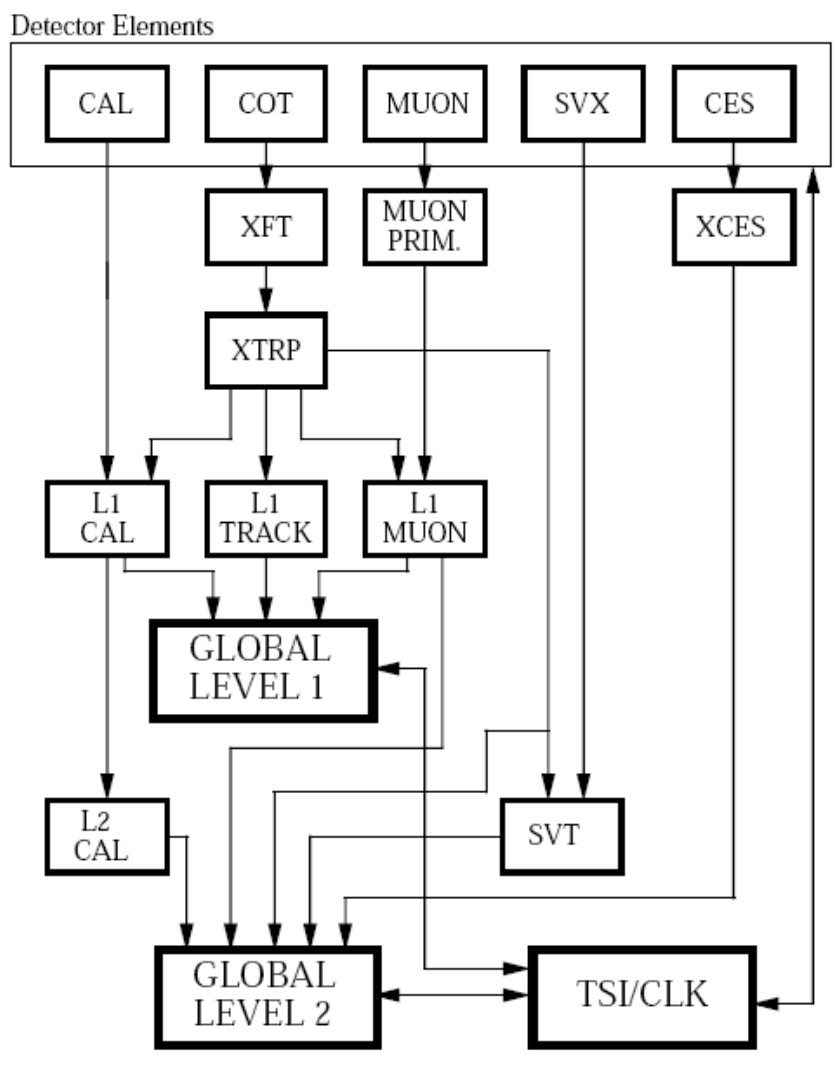

FiguRE 3.11: Block diagram of the Level 1 and the Level 2 trigger systems at CDF [44].

data are fed into $\mathrm{a} \sim 5 \mu s$ pipeline for processing. A fast charged particle tracking in the transverse plane is performed based on the four COT axial superlayers. Track candidates are then passed to the track extrapolator to find a matched calorimeter tower or muon stub. The L1 reduces the event rate from $1.7 \mathrm{MHz}$ down to $\sim 25 \mathrm{kHz}$.

The Level 2 trigger (L2) is a combination of hardware and software triggers. It uses the L1 event information in a more refined way. Additional information from the cluster finder (L2CAL), shower maximum detector in the central calorimeter (XCES) and the Silicon Vertex Track (SVT) are used to aid L2 decision. After the 
L2, the event rate is reduced to $400-1000 \mathrm{~Hz}$.

The Level 3 trigger (L3) consists of 16 processor farms with each containing 12 to 16 processor nodes. Events fed into L3 are reconstructed by the CDF offline reconstruction code using the full detector information with imposed loose cuts. This includes 3-dimensional track reconstruction, tighter matching of tracks to calorimeter and muon system. Event rate accepted at L3 is roughly $75 \mathrm{~Hz}$. 
4

\section{Measurement Strategy}

At the Tevatron, $W$ bosons are produced by $p \bar{p}$ collisions at a center-of-mass energy of $1.96 \mathrm{TeV}$ mainly through the following processes:

$$
\begin{aligned}
& u \bar{d} \rightarrow W^{+} \\
& \bar{u} d \rightarrow W^{-}
\end{aligned}
$$

About $80 \%$ of the time, a $W$ boson is produced when a valence quark from the proton or a valence antiquark from the antiproton is involved. The remaining $20 \%$ of the time a $W$ boson is produced by the annihilation of two sea quarks [45]. If $P_{1}$ $\left(P_{2}\right)$ is the 4-momentum of the proton (anti-proton) and $x_{1}\left(x_{2}\right)$ is the fraction of momentum carried by the partons undergoing the interaction, the energy available in the collision can be approximated as:

$$
\begin{gathered}
Q^{2}=\left(x_{1} P_{1}+x_{2} P_{2}\right)^{2} \approx 2 x_{1} x_{2} P_{1} P_{2} \approx 2 x_{1} x_{2}\left(E_{1} E_{2}+p_{1}^{z} p_{2}^{z}\right) \approx 4 x_{1} x_{2} E_{\text {beam }}^{2} \\
\Rightarrow Q \approx 2 \sqrt{x_{1} x_{2}} E_{\text {beam }}
\end{gathered}
$$

where $p_{1}^{z}=p_{2}^{z} \approx E_{1}=E_{2}=E_{\text {beam }}$. 
Since the momentum fraction carried by the colliding partons is inevitably unequal due to parton distribution functions (PDFs), the $W$ boson is typically produced with a net longitudinal momentum, which is unmeasured due to the finite detector acceptance down the beam pipe. Because of this, $W$ boson candidates are usually studied in the plane perpendicular to the beam direction, which is referred to as transverse plane in this thesis. The $W$ production is further complicated by associated initial-state gluon radiation, which boosts the $W$ in the transverse plane. The effects of PDFs and non-zero $W$ boson $p_{T}$ will be studied in Chapter 6 . As to the $W$ boson decay, there are three leptonic modes $\left(e \nu_{e}, \mu \nu_{\mu}\right.$ and $\left.\tau \nu_{\tau}\right)$ and two hadronic modes $(u \bar{d}$ and $c \bar{s})$ available. The $t \bar{b}$ hadronic decay mode is kinematically forbidden since top quark mass $m_{t}$ is much heavier than $W$ boson mass $m_{W}$. Owing to the fact that $W$ leptonic decay has clean experimental signature, we choose $W \rightarrow \mu \nu$ decay to measure $m_{W}$ by using transverse mass $m_{T}$, muon transverse momentum $p_{T}$ and neutrino transverse momentum $p_{T}^{\nu}\left(\right.$ or $\left.\not p_{T}\right)$. A similar analysis is also carried out by the CDF $W$ mass analysis group using the $W \rightarrow e \nu$ channel. Figure 4.1 illustrates $p \bar{p} \rightarrow W X \rightarrow \mu \nu X$ process. An axial view of a typical $W \rightarrow \mu \nu$ event in the CDF detector is shown in Figure 4.2.

Distributions of the chosen kinematic quantities have a common feature - Jacobian edge. The Jacobian edge is the region that carries most of the $W$ mass information and thus crucial for a precise $m_{W}$ measurement. In this chapter, we will first gain some insight on how the Jacobian edge arises in our chosen kinematic quantities, then we will give an overall description of our measurement strategy and introduce the techniques used in extracting $m_{W}$. 


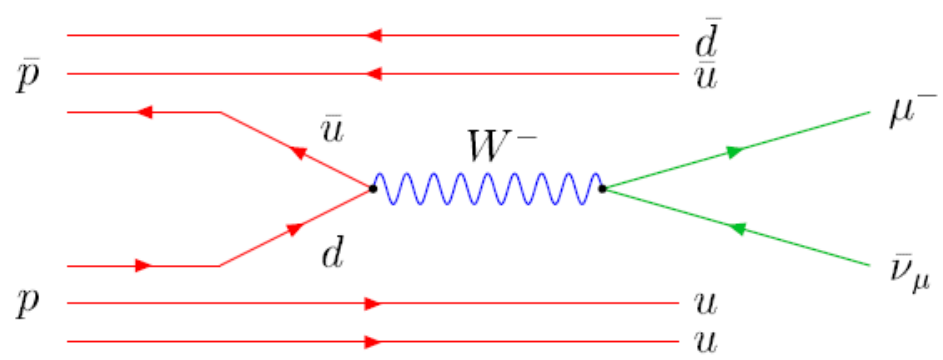

Figure 4.1: Leading order production and leptonic decay of a $W$ boson at the Tevatron.

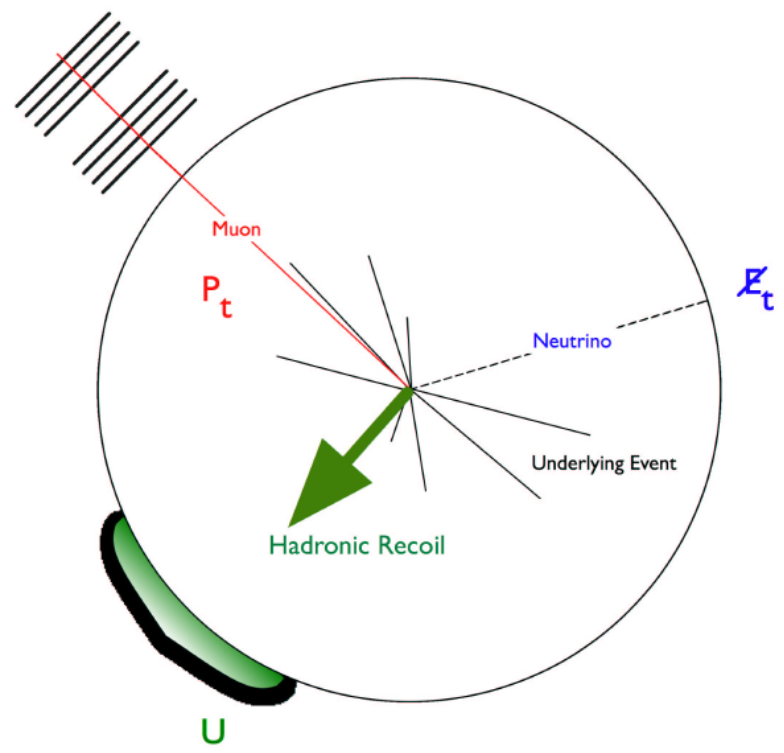

Figure 4.2: Axial view of a $W \rightarrow \mu \nu$ event in the CDF detector. The hadronic recoil deposits energy in calorimeter, the muon track leaves hits in the muon chambers and the neutrino escapes detection [59]. 


\subsection{Jacobian Edge}

At lowest order, the angular distribution of the muon from the $W$ decay is given by $[45]$ :

$$
\frac{d \sigma}{d \cos \hat{\theta}}=\sigma_{0}(\hat{s})(1-\lambda \cos \hat{\theta})^{2}
$$

where $\sigma_{0}$ is a factor independent of $\lambda$ and $\hat{\theta}$. $\lambda$ is the helicity of the $W$ boson with respect to the proton direction, $\hat{\theta}$ is the angle between the charged muon and the proton direction in the rest frame of $W$ (see Figure 4.3). Summing over $\mu^{+}$and $\mu^{-}$, we have

$$
\begin{aligned}
\frac{d \sigma}{d \cos \hat{\theta}} & =\sigma_{0}(\hat{s})\left[\frac{1}{2}(1+\cos \hat{\theta})^{2}+\frac{1}{2}(1-\cos \hat{\theta})^{2}\right] \\
& =\sigma_{0}(\hat{s})\left(1+\cos ^{2} \hat{\theta}\right)
\end{aligned}
$$

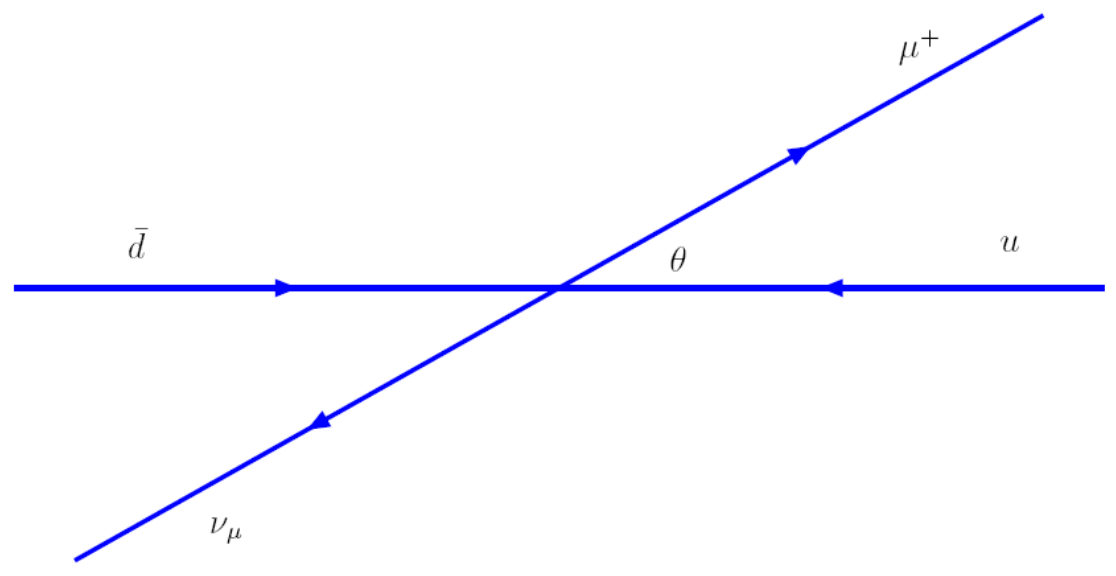

Figure 4.3: $W^{+}$decay in the center of mass frame.

If $W$ boson is assumed to be produced at rest and its decay products are treated as massless, then we have $p_{T}=\frac{1}{2} m_{W} \sin \hat{\theta}$. This leads to the differential cross-section 
with respect to muon $p_{T}$ :

$$
\begin{aligned}
\frac{d \sigma}{d p_{T}} & =\frac{d \sigma}{d\left(\left(m_{W} / 2\right) \sin \hat{\theta}\right)} \\
& =\frac{2}{m_{W}} \frac{d \sigma}{d \sin \hat{\theta}} \\
& =\frac{2}{m_{W}} \frac{d \sigma}{d \cos \hat{\theta}}\left|\frac{d \cos \hat{\theta}}{d \sin \hat{\theta}}\right| \\
& =\frac{2}{m_{W}} \sigma_{0}(\hat{s})\left(1+\cos ^{2} \theta\right)|\tan \hat{\theta}| \\
& =\sigma_{0}(\hat{s}) \frac{4 p_{T}}{m_{W}^{2}}\left(2-4 p_{T}^{2} / m_{W}^{2}\right)\left(\frac{1}{\sqrt{1-4 p_{T}^{2} / m_{W}^{2}}}\right)
\end{aligned}
$$

where $\left(1-4 p_{T}^{2} / m_{W}^{2}\right)^{-1 / 2}$ in the last equation comes from the Jacobian transform $\left|\frac{d \cos \hat{\theta}}{d \sin \hat{\theta}}\right|$. We can transfer $\frac{d \sigma}{d p_{T}}$ to $\frac{d \sigma}{d m_{T}}$ by using $m_{T}=2 p_{T}$ :

$$
\begin{aligned}
\frac{d \sigma}{d m_{T}} & =\frac{1}{2} \frac{d \sigma}{d p_{T}} \\
& =\sigma_{0}(\hat{s}) \frac{m_{T}}{m_{W}}\left(2-\frac{m_{T}^{2}}{m_{W}^{2}}\right)\left(\frac{1}{\sqrt{1-m_{T}^{2} / m_{W}^{2}}}\right)
\end{aligned}
$$

We can see from Eqn. (4.7) and Eqn. (4.8) that, for a fixed $m_{W}$ value, when $p_{T} \rightarrow \frac{m_{W}}{2}\left(m_{T} \rightarrow m_{W}\right), \frac{d \sigma}{d p_{T}}\left(\frac{d \sigma}{d m_{T}}\right)$ will tend to infinity. However, since $m_{W}$ is distributed accroding to a Breit-Wigner distribution rather than a delta function, infinity will never be reached. Take $\frac{d \sigma}{d p_{T}}$ as an example, the peak location at $p_{T}=\frac{m_{W}}{2}$ will be smeared by the natural width of $W\left(\Gamma_{W}\right)$ in an asymmetric way such that edge falls off rapidly above $\frac{m_{W}}{2}$ but relatively slowly below $\frac{m_{W}}{2}$. This rapidly falling edge is referred to as Jacobian edge since it originates from the Jacobian transformation. The asymmetry in the falling edge beyond and below $\frac{m_{W}}{2}$ is caused by kinematic constraints in $p_{T}$ since they can be no more than $\frac{m_{W}}{2}$ in the rest frame of $W$. The 
same argument applies to the Jacobian edge in $\frac{d \sigma}{d m_{T}}$. The Jacobian edge, which contains most of the $W$ mass information, is further smeared by non-zero $W$ boson $p_{T}$ and imperfect detector resolution.

\subsection{Strategy of this Measurement}

As mentioned earlier, the probabilistic property of momenta of the annihilating partons makes the longitudinal net momentum undeterminable on an event-to-event basis. For each event, we thus have to exclude the usage of momentum conservation along the beam direction and cannot use the 3 -d invariant mass spectrum for $m_{W}$ measurement. However, information in the transverse plane, which is perpendicular to the beam axis $z$, can be used to extract $m_{W}$ since the interacting $p$ and $\bar{p}$ has zero net transverse momentum. A two-dimensional transverse mass $m_{T}$ can thus be constructed using transverse information only:

$$
\begin{aligned}
m_{T} & =\sqrt{\left(E_{T}^{l}+E_{T}^{\nu}\right)^{2}-\left(\vec{p}_{T}^{l}+\vec{p}_{T}^{\nu}\right)^{2}} \\
& =\sqrt{2 p_{T}^{\mu} p_{T}^{\nu}(1-\cos \Delta \phi)}
\end{aligned}
$$

where $\Delta \phi$ is the angle between $\mu$ and $\nu$ in the transverse $(r-\phi)$ plane. $p_{T}^{\mu}$ and $p_{T}^{\nu}$ is magnitude of the transverse momentum of $\mu$ and $\nu$, respectively. Whether we can determine $m_{T}$ precisely depends on how well $p_{T}^{\mu}$ and $p_{T}^{\nu}$ can be measured. The $m_{T}$ spectrum, however, cannot be predicted analytically due to detector resolution, transverse motion of the $W$ boson at production and incomplete detector coverage.

To extract the $W$ boson mass from the $m_{T}$ distribution, different transverse mass distributions are simulated for a range of input $m_{W}$ mass values. After adding measured contribution from backgrounds, these generated transverse mass distributions are used as templates (800 in this analysis) to compare with the transverse mass distribution obtained from data. Using the binned likelihood maximization method 
(see Sec. 4.3), the template which matches best to the data distribution gives the best-fit $W$ boson mass.

There are two key aspects for a precise $W$ mass measurement. Firstly, the detector needs to calibrated to the best possible level such that any left-over data bias is relatively small compared with the measurement precision to be reached. Calibration is a process to adjust measured quantities such that some precisely known properties of particles like mass can be reproduced as close as possible. Since the charged muon carries most of the observable mass information in the $W \rightarrow \mu \nu$ decay, a precise calibration of the muon momentum is needed. We use large samples of $J / \psi \rightarrow \mu \mu$ and $\Upsilon \rightarrow \mu \mu$ data events, which are fully reconstructable with well known resonance masses, to precisely calibrate the muon momentum. As a cross check, we apply the obtained calibration to the $Z \rightarrow \mu \mu$ events to measure the $Z$ boson mass. The accuracy of the momentum calibration from $J / \psi$ and $\Upsilon$ analyses can be demonstrated if the measured $Z$ boson mass $m_{Z}$ is consistent with the PDG value. If momentum calibrations from all $J / \psi \rightarrow \mu \mu, \Upsilon \rightarrow \mu \mu$ and $Z \rightarrow \mu \mu$ samples are consistent, they can be further combined to give the most precise momentum calibration.

Secondly, we need to simulate the $W$ boson production and decay processes as well as detailed detector effects on the decay products as precisely as possible. For example, the neutrino transverse momentum $p_{T}^{\nu}$, which cannot be measured directly, can be inferred in an indirect way via momentum conservation in the transverse plane, i.e., $p_{T}^{\nu}$ can be calculated from $\vec{p}_{T}^{\nu}+\vec{p}_{T}^{\mu}+\vec{U}_{T}^{W}=0$, where $\vec{U}_{T}^{W}$ is the recoil balanced against the transverse motion of the produced $W$ boson. Once $\vec{p}_{T}^{\mu}$ is known, we need $\vec{U}_{T}^{W}$ to infer $\vec{p}_{T}^{\nu}$, Since the $Z$ boson has a similar mass compared with the $W$ boson and $Z \rightarrow \mu \mu$ events have the advantage that transverse momenta of the two decay muons can be measured precisely, we use $Z \rightarrow \mu \mu$ decays to measure the recoil energy $\vec{U}{ }_{T}^{Z}$ against produced $Z$ boson. The inferred $\vec{U}_{T}^{Z}$ and the energy measurement of the 
recoil from the calorimeter $\vec{u}_{T}^{Z}$ can be used to derive a calorimeter response function. This function can thus be applied to $W \rightarrow \mu \nu$ events to get $\vec{U}_{T}^{W}$. This shows the recoil model enters Eqn. (4.9) indirectly in the neutrino transverse momentum inference and thus needs to be well parameterized for a precise $m_{W}$ measurement.

The variables $p_{T}^{\mu}$ and $p_{T}^{\nu}$ are the other two main observables used in our $m_{W}$ measurement. The $p_{T}^{\mu}$ is complementary to the $m_{T}$ in its response to the detector effects and the $W$ boson production dynamics. As illustrated in Figure 4.4, the $m_{T}$ observable is relatively insensitive to the transverse motion of the produced $W$ boson. It is however sensitive to the detector effects through $p_{T}^{\nu}$ which is subject to the recoil resolution. In contrast, the $p_{T}^{\mu}$ observable is relatively insensitive to detector effects but sensitive to the $W$ boson production kinematic effects. The $p_{T}^{\nu}$ is used as a cross-check since it is sensitive to both the recoil momentum measurement and the $W$ boson $p_{T}$ spectrum. The $m_{W}$ measurement from $p_{T}^{\nu}$ spectrum thus has the largest systematic uncertainty compared to the measurements from $m_{T}$ and $p_{T}^{\mu}$ spectra. The same binned maximum likelihood fitting method is applied to $p_{T}^{\mu}$ and $p_{T}^{\mu}$ distributions to extract $m_{W}$. In the last step, the fitted $m_{W}$ from $m_{T}, p_{T}^{\mu}$ and $\not p_{T}$ are combined to give the final $m_{W}$ result.

\subsection{Template Likelihood Fits}

We perform mass measurements by using a template binned likelihood fitting procedure to find the maximum likelihood estimation (MLE). The mass templates are generated with different input mass values then smeared appropriately by simulated detector effects to mimic the distributions from the data. We fit distributions from the simulated templates against the corresponding distribution from data to extract the most probable mass value. The likelihood is given by the products of the Poisson probability across all the bins within the chosen fitting window, where the Poisson 

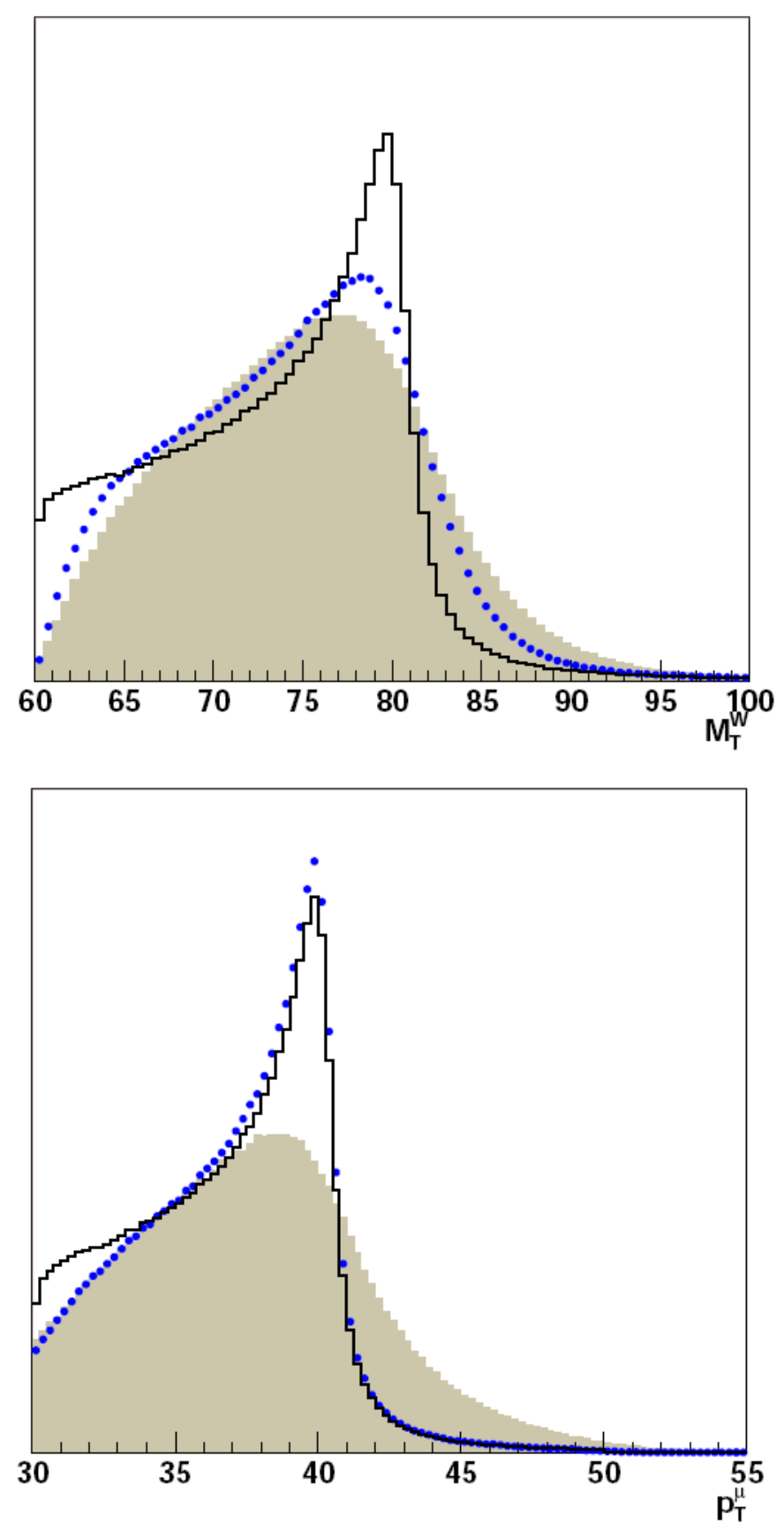

Figure 4.4: Illustrations of $m_{T}$ (top) and $p_{T}^{\mu}$ (bottom) spectra to $p_{T}^{W}$ and detector effects. Shaded area is for both finite detector resolution and $p_{T}^{W} \neq 0$; blue points is for finite detector resolution with $p_{T}^{W}=0$; black histrogram is for perfect detector resolution with $p_{T}^{W} \neq 0[59]$. 
probability is the probability of observing $n_{i}$ data events within the bin in consideration given $m_{i}$ expected events in the corresponding bin from simulation:

$$
L=\prod_{i=1}^{N} \frac{e^{-m_{i}} m_{i}{ }^{n_{i}}}{n_{i} !}
$$

where $N$ is the total number of bins in the fitting window. By taking the logarithm we get:

$$
\ln L=\sum_{i=1}^{N}\left(n_{i} \ln m_{i}-m_{i}-\ln \left(n_{i} !\right)\right)
$$

For the ease of computation, we can approximate Eqn. (4.12) by using Sterling's approximation

$$
\ln n ! \approx n \ln n-n
$$

which leads to

$$
\ln L \approx \sum_{i=1}^{N}\left(n_{i} \ln m_{i}-m_{i}-n_{i} \ln n_{i}+n_{i}\right)
$$

The best-fit value maximizes $\ln L$ (or equivalently minimizes $-\ln L$ ). When the size of data sample is large, the $\pm 1 \sigma_{\text {stat }} m_{W}$ values are given by $-\ln L=-\ln L_{\max }+$ $1 / 2$ when the variance of the MLE reaches the Cramer-Rao lower bound [47]. The $1 \sigma$ value is symmetrized by averaging $+1 \sigma$ and $-1 \sigma$ values. To get the final single $m_{W}$ in $W \rightarrow \mu \nu$ channel, we perform the fitting separately on each of the $m_{T}, p_{T}^{\mu}$ and $E_{T}$ (or $p_{T}^{\nu}$ ) distributions, then combine the three extracted $m_{W}$ values using the corresponding correlation coefficients.

One thing to note is that the asymptotically optimal properties of the MLE guarantees the measured $m_{W}$ and its associated statistical uncertainty to be unbiased for an infinite data sample. For a finite but large data sample as our $2.2 \mathrm{fb}^{-1} W$ 
data, $Z$ data, $J / \psi$ data and $\Upsilon$ data, the introduced bias by MLE is found to be negligible, see reference [48] for details.

\subsection{Blinding Technique}

A blind analysis is a technique used to prevent the analyzer from looking at the answer while performing a measurement. It is an effective way to reduce the analyzer's bias of favoring the experimental result in a particular direction.

To minimize the intended or unintended bias caused by favoring the world averaged $W$ boson mass published in PDG $\left(m_{W}=80.399 \pm 0.023 \mathrm{GeV}[32]\right)$, we hide the central value of measured $m_{W}$ from the analyzers (us) and the rest of the CDF Collaboration by adding a certain offset which is randomly drawn from a uniform distribution within $[-75 \mathrm{MeV}, 75 \mathrm{MeV}]$. Neither statistical nor systematic uncertainties would be obscured by the blinding. For ease of comparison, the same offset is added to the measured $m_{W} \mathrm{~s}$ from the three variables $m_{T}, p_{T}^{\mu}$ and $p_{T}^{\nu}$. The same offset is also applied to the measured $m_{W}$ s from both the electron channel $(W \rightarrow e \nu)$ and the muon channel $(W \rightarrow \mu \nu)$ such that cross checks can be carried out.

Another randomly drawn offset, which is different from the random offset added to $m_{W}$, is added to the measured $m_{Z}$ s from both the electron channel $(Z \rightarrow e e)$ and the muon channel $(Z \rightarrow \mu \mu)$ such that we can compare $m_{Z}$ results between two channels as well as with the $m_{Z}$ PDG value before we study the $W$ s. Other parts of the analysis, including momentum scale measurement and EM calorimeter energy scale measurement via $E / p$ distribution, are not blinded. 


\section{5}

\section{Dataset and Event Selection}

The data used in this $W$ boson mass measurement were collected at the CDF detector during Run II of the Tevatron between February 4, 2002 and August 4, 2007. The integrated luminosity of the $W \rightarrow \mu \nu$ data ${ }^{1}$, excluding runs that have been identified as bad, is $\approx 2.2 \mathrm{fb}^{-1}$. This dataset includes the $200 \mathrm{pb}^{-1}$ sample used in the last CDF $W$ mass publication [8].

Several additional datasets collected during the same data-taking period are used for various purposes related to the $W$ boson mass measurement. The $J / \psi \rightarrow \mu \mu$ and $\Upsilon \rightarrow \mu \mu$ datasets are used to calibrate the momentum scale of the COT detector. The di-muon decays of the $Z$ boson, whose mass is of the same order as that of the $W$ boson, comprise an ideal control sample for modelling the energy recoiling against the $W$ boson since the two decaying muons from the $Z$ can be fully reconstructed, allowing the recoil model parameters to be studied in detail. The $Z \rightarrow \mu \mu$ sample can

1 We use SAMLumi tool provided by the CDF good run list page at http://wwwcdf.fnal.gov/internal/dqm/goodrun/good.html to calculate the integrated luminosity. Usage: ./SAMLumi bhmu goodrun.txt. The SAMLumi-calculated integrated luminosity needs to be corrected by a scale factor of 1.019 . The total luminosity uncertainty is $5.8 \%$, including $4.2 \%$ uncertainty from the CLC measurement [9] and $4.0 \%$ uncertainty from inelastic $p \bar{p}$ cross section measurement [10]. This translates into a total $W \rightarrow \mu \nu$ integrated luminosity of $2.18 \pm 0.13 \mathrm{fb}^{-1}$. 
also be used to measure tracking hit resolution and as a cross-check of the momentum scale measured from $J / \psi \rightarrow \mu \mu$ and $\Upsilon \rightarrow \mu \mu$ samples. A sample of minimum bias events, which are triggered by requiring coincident hit in scintillator counters located at $\sim 6 \mathrm{~m}$ from the interaction point, is used to determine parameters of the recoil model. Cosmic ray data from the same period are used for internal alignment of the COT. Table 5.1 shows the numbers of $J / \psi \rightarrow \mu \mu, \Upsilon \rightarrow \mu \mu, Z \rightarrow \mu \mu$ and $W \rightarrow \mu \nu$ events after applying the selection cuts presented in Section 5.1 and Section 5.2.

Table 5.1: Some datasets used in this $W$ mass analysis. The numbers are after all cuts have been applied.

\begin{tabular}{lc}
\hline \hline Sample & Number of Candidate Events \\
\hline$J / \psi \rightarrow \mu \mu$ & 5941152 \\
$\Upsilon \rightarrow \mu \mu$ & 238999 \\
$Z \rightarrow \mu \mu$ & 59738 \\
$W \rightarrow \mu \nu$ & 624708 \\
\hline \hline
\end{tabular}

\section{$5.1 \quad W \rightarrow \mu \nu$ and $Z \rightarrow \mu \mu$ Samples}

In our $W$ mass analysis, the $Z \rightarrow \mu \mu$ sample is used as a control sample in two ways. First, it is used to tune the parameters of the recoil model, which will be applied in our fast detector simulation (DukeSim) to describe the recoil against the $W$ boson production. Second, it is used to cross-check the momentum scale obtained from low mass $J / \psi$ and $\Upsilon$ resonances. To minimize the bias caused by different selection cuts, we choose the $W$ and $Z$ event selection as similarly as possible.

The $W$ boson event selection criteria are chosen such that a high quality data sample with low backgrounds can be obtained and the kinematics of the decaying muon and the neutrino can be well understood. A general purpose high $p_{T}$ muon trigger is used at Level 1 to select $W$ and $Z$ samples by requiring CMU or CMX $p_{T}>6 \mathrm{GeV}$ matched to an XFT track with $p_{T}>4 \mathrm{GeV}$ for CMUP or $p_{T}>8 \mathrm{GeV}$ 
for CMX. The XFT $p_{T}$ cut for CMUP candidates is raised to $p_{T}>8 \mathrm{GeV}$ at Level 2 to further reduce rates. At Level 3, the high-momentum muon trigger requires the muon candidate to have $p_{T}>18 \mathrm{GeV}$. The $W$ and $Z$ samples passing Level 3 are then fully reconstructed using CDF offline reconstruction software. For our $W$ mass analysis, we use CDF software version 6.1.4int11.

Before selecting a $W$ or $Z$ event, we first require the run number of the candidate event to be listed in a commmon good run list which is exactly the same for $J / \psi$, $\Upsilon, Z$ and $W$ samples. Our selection then starts with the two COT tracks with the highest $p_{T}$. For muon identification using these two high- $p_{T}$ tracks, we require at least one of the tracks to pass the muon identification requirements listed in Table 5.2. Additional event selection cuts (see Table 5.3 and Table 5.4) are applied to select $Z$ boson candidates and $W$ boson candidates, respectively.

Table 5.2: Muon candidate requirements.

\begin{tabular}{lc}
\hline \hline \multicolumn{2}{c}{ Muon Candidates } \\
\hline Variable & Requirement \\
\hline Geometric & Fiducial in CMUP or CMX \\
$|\Delta x|_{\text {CMU }}$ & $<3.0 \mathrm{~cm}$ \\
$|\Delta x|_{\text {CMP }}$ & $<5.0 \mathrm{~cm}$ \\
$|\Delta x|_{\text {CMx }}$ & $<6.0 \mathrm{~cm}$ \\
Track type & $\neq 3,28,29$ \\
Number of axial SL & $\geq 3$ with 5 hits each \\
Number of stereo SL & 3 with 5 hits each \\
Number of hits in axial SL & $\geq 25$ \\
Number of hits in stereo SL & $\geq 25$ \\
Track impact parameter $\left|\Delta d_{0}\right|$ & $<0.1 \mathrm{~cm}$ \\
Track originate in $\hat{z}\left|\Delta z_{0}\right|$ & $<0.0 \mathrm{~cm}$ \\
Energy deposition in EM $E_{\text {em }}$ & $<2.0+\max (0,0.0015(\mathrm{p}-100)) \mathrm{GeV}$ \\
Energy deposition in HAD $E_{\text {had }}$ & $<.0+\max (0,0.0028(\mathrm{p}-100)) \mathrm{GeV}$ \\
Track $p_{T}$ & $>30.0 \mathrm{GeV} / \mathrm{c}$ \\
$\chi^{2} /$ d.f. & $<3.0$ \\
Track through all COT layers & TRACK_trk_nonbc_lastLayerCT [track] $==95$ \\
Number of transitions $N_{\text {trans }}$ & $>30+2\left(\chi^{2} / \mathrm{df}\right)$ \\
\hline \hline
\end{tabular}


Table 5.3: Event requirements on $Z$ boson candidates.

\begin{tabular}{lc}
\hline \hline \multicolumn{2}{c}{$Z$ boson candidate requirements } \\
\hline Variable & Requirement \\
\hline$Z$ boson candidate $p_{T}^{\mu \mu}$ & $<30.0 \mathrm{GeV} / \mathrm{c}$ \\
Di-muon invariant mass $m_{\mu \mu}$ & $66 \mathrm{GeV} / \mathrm{c}^{2}<m_{\mu \mu}<116 \mathrm{GeV} / \mathrm{c}^{2}$ \\
$p_{T}^{\mu_{1}}$ and $p_{T}^{\mu_{2}}$ & $<65.0 \mathrm{GeV} / \mathrm{c}$ \\
Standard cosmic veto & $<4.0 \mathrm{~ns}$ \\
$\left|\Delta t_{0}\right|$ & $<$ \\
\hline \hline
\end{tabular}

Table 5.4: Event requirements on $W$ boson candidates.

\begin{tabular}{lc}
\hline \hline \multicolumn{2}{c}{$W$ boson candidate requirements } \\
\hline Variable & Requirement \\
\hline$E_{T}$ & $>30.0 \mathrm{GeV} / \mathrm{c} ;<55.0 \mathrm{GeV} / \mathrm{c} ;$ \\
$|u|$ & $<15.0 \mathrm{GeV} ;$ \\
Transverse mass $m_{T}$ & $>60.0 \mathrm{GeV} / \mathrm{c}^{2} ;<100.0 \mathrm{GeV} / \mathrm{c}^{2}$ \\
Muon $p_{T}$ & $<55.0 \mathrm{GeV} / \mathrm{c}$ \\
Standard cosmic veto & \\
Reject $Z$-like events & \\
\hline \hline
\end{tabular}

In the muon identification requirements, the variable $|\Delta x|$ is used to quantify the matching extent in the $r-\phi$ plane between the reconstructed positions of the muon chamber stubs and the extrapolated positions of COT tracks in the muon detector. We only use track types which are good and relevant to our analysis by rejecting tracks reconstructed with Inside-Out algorithm [49] (TrkType=3 [50]) and Backward-Outside-In algorithm (TrkType=28,29 [50]). The candidate track is required to traverse all the COT layers with at least 3 axial SLs and at least 3 stereo SLs fired, where a fired SL is defined to have at least 5 hits within it. The 25/25 cuts on the total number of hits in axial/stereo superlayers are applied consistently to all our four datasets: $J / \psi, \Upsilon, Z$ and $W$. For the muon channel, the impact parameter of the reconstructed track is required to be within $0.1 \mathrm{~cm}$ in $r-\phi$ plane with respect 
Table 5.5: $Z$ boson identification for rejection in $W$ boson event selection

\begin{tabular}{l}
\hline \hline$Z$ identification for rejection in $W$ event selection \\
\hline 1$).$ The first muon is identified \\
2). A second opposite-charged muon candidate passing: \\
a). Muon identification requirements. \\
OR \\
b). The following looser set of cuts: \\
$p_{T}>20.0 \mathrm{GeV} / \mathrm{c} ;$ \\
$\geq 3$ axial SLs with 5 hits each \\
$\geq 3$ stereo SLs with 5 hits each \\
Track type $\neq 3,28,29$ \\
$\chi^{2} /$ d.f. $<3.0$ \\
$\left|\Delta d_{0}\right|<0.1$ \\
$E_{\text {em }}<2+\max (0,0.0015(\mathrm{p}-100))$ \\
$E_{\text {had }}<6+\max (0,0.0028(\mathrm{p}-100))$ \\
\hline \hline
\end{tabular}

to the beamline. The track origin in $z$ is required to be less than $60 \mathrm{~cm}$ away from the center of the CDF detector along the $z$ direction. The energy depositions in the electromagnetic calorimeter and the hadronic calorimeter by the extrapolated COT track are measured separately. We require the measured energy depositions $E_{\text {em }}$ and $E_{\text {had }}$ with $p$-dependent correction applied to be less than $2 \mathrm{GeV}$ and $6 \mathrm{GeV}$, respectively, since the muon ionization is greatly suppressed by its relatively big mass compared with electrons. We also require the muon candidate to have good track fit quality by imposing $\chi^{2} /$ d.f. $<3.0$. A new muon identification variable - the number of transitions $N_{\text {trans }}$ - is used in our event selection. $N_{\text {trans }}$ counts how many times the residuals of a COT track change signs. By using the fact that $N_{\text {trans }}$ tends to be high for promptly produced muons while low for muons from the decays of longlived hadrons (i.e. "decay-in-flight"), the 2-dimensional cut on $N_{\text {trans }}$ as a function of $\chi^{2} / \mathrm{df}$ is applied to remove such muon background.

We first check if the candidate event is a $Z$ event by requiring the first track to meet all the above requirements as listed in Table 5.2, then the second track is 
identified with a less restrictive criteria by dropping the muon stub requirements (i.e. dropping fiducial stub identifier in CMU and CMP or CMX, and dropping the cuts on track-stub matching $|\Delta x|$ ). However, we add requirement on the charge of the second track to be opposite to the charge of the first muon candidate. Several additional cuts in Table 5.3 are applied at the $Z$ candidate event level to select high quality $Z$ boson sample. The di-lepton transverse momentum $p_{T}^{\mu \mu}$ is restricted to be less than $30 \mathrm{GeV} / \mathrm{c}$ and an additional $p_{T}$ upper bound with $p_{T}<65.0 \mathrm{GeV} / \mathrm{c}$ is applied to both $Z$ muon candidates to make it consistent with the lepton candidates from $W$ bosons. Since cosmic ray events can mimic muons by leaving hits in both the COT and the muon chambers, a cosmic ray tagger based on COT hit timing information is used to remove such events from both $Z$ sample and $W$ sample.

Our selection then moves on to check if the event with an identified muon is a $W$ boson by requiring it to pass various kinematic cuts. We take $30.0 \mathrm{GeV}<\not_{T}<55.0$ $\mathrm{GeV}$ for total missing transverse energy, $60.0 \mathrm{GeV}<m_{T}<100.0 \mathrm{GeV}$ for transverse mass, $30.0 \mathrm{GeV}<p_{T}<55.0 \mathrm{GeV}$ for muon transverse momentum and $\left|u_{T}\right|<15$ $\mathrm{GeV}$ for the recoil energy. The narrow kinematic regions are chosen to significantly suppress the backgrounds while retaining events with precise $m_{W}$ information. The same standard cosmic ray tagger is applied to remove the cosmic ray events from $W$ candidates. To reduce $Z / \gamma^{*} \rightarrow \mu \mu$ background in the $W \rightarrow \mu \nu$ sample where one of the two decaying muons is not reconstructed thus mimicing a neutrino, we remove from $W$ candidates the events which pass the $Z$ identification requirements listed in Table 5.5.

\section{$5.2 J / \psi \rightarrow \mu \mu$ and $\Upsilon \rightarrow \mu \mu$ Samples}

One crucial part for this $W$ boson mass measurement at the $0.03 \%$ level is a precise momentum calibration. $J / \psi \rightarrow \mu \mu$ and $\Upsilon \rightarrow \mu \mu$ decays have the advantage that they are fully reconstructable and the masses of $J / \psi$ and $\Upsilon$ are precisely measured 
by other experiments. Besides that, the large production of $J / \psi \rightarrow \mu \mu$ and $\Upsilon \rightarrow \mu \mu$ quarkonium decays at CDF make them ideal control samples to precisely set the momentum scale for the $W$ boson mass measurement. Specifically, the $J / \psi \rightarrow \mu \mu$ sample can be used to measure the magnetic field non-uniformity within the COT, to determine the track energy loss upstream of the COT and to quantify the effects of the COT misalignment. The $\Upsilon \rightarrow \mu \mu$ sample can also be used to calibrate the COT as a cross-check. Additionally, it allows us to study the differences between using beam-constrained tracks and using non-beam-constrained tracks.

We use the $J / \psi$ and $\Upsilon$ datasets corresponding to the same data-taking period as the $W$ dataset between February 2002 and August 2007 for the momentum scale measurement. The secondary dimuon datasets jpmm0d, jpmm0h, jpmm0i and part of jpmm0 $\mathrm{j}$ are used to select $J / \psi \rightarrow \mu \mu$ events. The dimuon datasets jbmm0d, jbmm0h, jbmm0 $i$ and part of $\mathrm{jbmm0j}$ are used to select $\Upsilon \rightarrow \mu \mu$ events. The samples are reconstructed using CDF-software release version 6.1.4int11 with the latest $z$-dependent COT alignment. The same good run list used in the $W$ and $Z$ samples is also applied to the $J / \psi$ and $\Upsilon$ samples.

\subsection{1 $\mathrm{J} / \psi \rightarrow \mu \mu$ Data Sample}

The trigger requirements for the $J / \psi$ sample collection are illustrated in Table 5.6. Two XFT tracks are required to have matched muon stubs when extrapolated into the muon chambers. At Level 1, one of the XFT tracks is required to have $p_{T}>$ $1.5 \mathrm{GeV} / \mathrm{c}$ with a matching CMU segment. The other is required to have $p_{T}>1.5$ $\mathrm{GeV} / \mathrm{c}$ if it is also matched with a CMU segment, or have $p_{T}>2.0 \mathrm{GeV} / \mathrm{c}$ if matched with a CMX segment. The level $2 \mathrm{~J} / \psi$ trigger does not impose any additional cuts. At the Level 3, the two tracks are required to have opposite charge with the di-muon invariant mass within $2.7 \mathrm{GeV}$ and $4.0 \mathrm{GeV}$. Additionally, their z vertex positions are required to be consistent with each other within $5 \mathrm{~cm}$ distance $\left(\left|\Delta z_{0}\right|<5 \mathrm{~cm}\right)$. A 
full list of triggers used in $J / \psi$ sample is given in Table 5.7. The $J / \psi$ muon selection requirements are summarized in Table 5.8.

Table 5.6: Trigger requirements for $J / \psi$ sample

\begin{tabular}{cc}
\hline \hline & Trigger Level Requirements \\
\hline Level 1 & two CMU tracks with $p_{T}>1.5 \mathrm{GeV} / \mathrm{c}$ \\
& $\underline{\mathrm{OR}}$ \\
& one CMU track with $p_{T}>1.5 \mathrm{GeV} / \mathrm{c}$, \\
& one CMX track with $p_{T}>2.0 \mathrm{GeV} / \mathrm{c}$ \\
\hline Level 3 & opposite charge \\
& $2.7 \mathrm{GeV} / \mathrm{c}^{2}<m_{\mu \mu}<4.0 \mathrm{GeV} / \mathrm{c}^{2}$ \\
$\left|\Delta z_{0}\right|<5.0 \mathrm{~cm}$ \\
\hline \hline
\end{tabular}

Table 5.7: List of trigger tables for $J / \psi$ sample

\begin{tabular}{ll}
\hline \hline & Trigger Tables \\
\hline CMUP-CMU & JPSI_CMUCMU1.5 \\
& JPSI_CMUCMU1.5_DPS \\
& JPSI_CMUCMU2 \\
& JPSI_CMUCMU2_PS2 \\
& JPSI_CMUCMU2_PS10 \\
& JPSI_CMUCMU2_PS50 \\
\hline CMUP-CMX & JPSI_CMU1.5_CMX2 \\
& JPSI_CMU1.5_CMX2_DPS \\
& JPSI_CMU2_CMX2_PS2 \\
& JPSI_CMU2_CMX2_PS10 \\
& JPSI_CMU2_CMX2_PS50 \\
\hline \hline
\end{tabular}

\subsection{2 $\Upsilon \rightarrow \mu \mu$ Data Sample}

The $\Upsilon \rightarrow \mu \mu$ decay sample, which although has smaller statistical power compared with $J / \psi \rightarrow \mu \mu$ decay sample, can be used as a bridge to study the beam-constrained and non-beam-constrained tracking options since $\Upsilon \rightarrow \mu \mu$ events are promptly produced. The $\Upsilon$ resonance, with a mass at the order of $10 \mathrm{GeV}$, also acts an inter- 
Table 5.8: Muon candidate requirements for $J / \psi$ and $\Upsilon$ samples.

\begin{tabular}{lcc}
\hline \multicolumn{3}{c}{ Muon Candidates for $J / \psi$ and $\Upsilon$ Samples } \\
\hline Sample & $J / \psi$ & $\Upsilon$ \\
\hline Transverse momentum $p_{T}$ & $>2.2 \mathrm{GeV}$ & $>4.2 \mathrm{GeV}$ (CMUP) \\
& & $>3.2 \mathrm{GeV}(\mathrm{CMU} / \mathrm{CMX})$ \\
Number of axial SL & 4 with 7 hits each & $\geq 3$ with 5 hits each \\
Number of stereo SL & 4 with 7 hits each & $\geq 3$ with 5 hits each \\
Number of hits in axial SL & & $\geq 25$ \\
Number of hits in stereo SL & & $\geq 25$ \\
Track impact parameter $\left|\Delta d_{0}\right|$ & $<0.3 \mathrm{~cm}$ & $<0.3 \mathrm{~cm}$ \\
$z$ differences of two muon $\left|\Delta z_{0}\right|$ & $<3.0 \mathrm{~cm}$ & $<3.0 \mathrm{~cm}$ \\
\hline \hline
\end{tabular}

mediate reference point between the 3 times lighter $J / \psi$ resonance and the 9 times heavier $Z$ boson to study the momentum scale as a function of energy since we want to extrapolate the momentum scale obtained from the low mass resonances to the high mass $W$ boson.

The Level 1 trigger requirements for the selection of $\Upsilon$ sample is the same as those for the $J / \psi$ sample. The Level 3 requires dimuon candidate events to have one muon with $p_{T}>4.0 \mathrm{GeV} / \mathrm{c}$ and matching segments in CMU and CMP. The other muon candidate is required to have opposite charge with $p_{T}>3.0 \mathrm{GeV} / \mathrm{c}$ and matching segments in either CMU or CMX. Events with dimuon invariant mass between 8.0 $\mathrm{GeV} / \mathrm{c}^{2}$ and $12.0 \mathrm{GeV} / \mathrm{c}^{2}$ are accepted. Similar to the selection of $J / \psi$ sample, the $p_{T}$ cuts are raised by $0.2 \mathrm{GeV} / \mathrm{c}$ at offline stage to avoid possible $p_{T}$ threshold biases. We further require each track to have at least 5 hits in at least 3 axial and 3 stero superlayers with impact parameter $\left|d_{0}\right|<0.3 \mathrm{~cm}$. To be consistent with $W$ and $Z$ sample selections, we require the total number of hits from both axial and stero superlayers to be at least 25 each. The two tracks are required to originate from a common vertex with $\left|\Delta z_{0}\right|<3.0 \mathrm{~cm}$. The muon candidate requirements for $\Upsilon$ sample is shown in Table 5.8. Two separate $\Upsilon \rightarrow \mu \mu$ data samples are obtained by using 
beam-constrained tracks and by using non-beam-constrained tracks, respectively. 


\section{6}

\section{$W$ Boson Production and Decay Model}

Since we extract $m_{W}$ by fitting $m_{T}, p_{T}^{\mu}$ and $p_{T}^{\nu}\left(p_{T}^{\prime}\right)$, whose simulated spectra will be altered for variations of $W$ boson production and decay models, we need to understand how a $W$ boson is produced in a $p \bar{p}$ collision and how it decays to a charged lepton plus a neutrino via weak interaction. There are theoretical calculations which describe the relevant processes. The QCD uncertainties on $m_{W}$ arise from two parts: the model for the $W$ boson's recoil in the transverse plane and parton distributions. The QED uncertainties on $m_{W}$ come from real photon radiation. In this chapter, we will explore the associated uncertainties due to those theoretical descriptions.

\subsection{Parton Distribution Functions (PDF)}

The partonic structure of hadrons plays an important role in the $W$ mass measurement. A parton distribution fuction $(\mathrm{PDF})$ is defined as the probability to find a parton $i$ which carries a momentum fraction $x_{i}$ at momentum transfer $Q^{2}$. PDFs have been parameterized for quarks, anti-quarks and gluons inside a proton, and the parameterizations are constrained by global analyses of hadron-hadron and lepton- 
hadron collision data including deep inelastic electron-proton scattering (DIS), $W$ boson charge asymmetry and jet production. PDFs enter into the $W$ boson mass measurement since the momenta of the interacting partons in $p \bar{p}$ collisions determine the longitudinal momentum of the produced $W$ boson, which affects the transverse mass $m_{T}$ distribution indirectly through cuts on the detector acceptance and cuts on the kinematics of the decay leptons. The dependence of $m_{T}$ on longitudinal momentum, seems contrary to the idea that $m_{T}$ is invariant under longitudinal boosts of the $W$ boson, is actually caused by incomplete detector coverage in rapidity.

Two collaborations, CTEQ [51] and MRST [52], independently perform global fits to data and provide regular updates to parton distribution fuctions. By allowing 20 free parameters in a global fit to data, CTEQ6 uses a Hessian matrix method [54] to explore the variation of $\chi_{\text {global }}^{2}$, which is calculated by using data sets from 15 different experiments, in the neighborhood of the global minimum in a 20-dimensional space. The $20 \times 20$ matrix is first diagonalized, then the 20 orthogonal eigenvectors are determined. A total of 40 PDFs which correspond to $90 \%$ coverage of the probability space are determined by positive and negative variation of each eigenvector such that $\Delta \chi_{\text {global }}^{2}=T^{2}$.

Statistically speaking, the tolerance $T^{2}$ should be chosen to 1 to determine the $1 \sigma$ uncertainty of a given parameter. However, when the data used in global fit are correlated and inconsistent with each other, the tolerance $T^{2}$ no longer has a statistical meaning. It just indicates a range of global fits which are determined to be acceptable. The CTEQ6 Collaboration chooses $T^{2}=100\left(\Delta \chi_{\text {global }}^{2} \leq 100\right)$ to obtain the uncertainty.

To estimate the systematic uncertainty on $m_{W}$ from the PDFs, we reweight $W$ boson events generated with RESBOS by using a PDF-interface in ROOT environment. The $W$ boson events are reweighted according to each of the 40 PDF error sets and are fed into the fast simulation to generate $m_{T}, p_{T}$ and $\not p_{T}\left(\mathbb{E}_{T}\right)$ templates. These 
templates are then fitted against the corresponding kinematic distribution generated using the default CTEQ6M template (the central PDF set). The variation of the fitted mass difference in terms of 20 different eigenvector pairs is shown in Fig. 6.1.

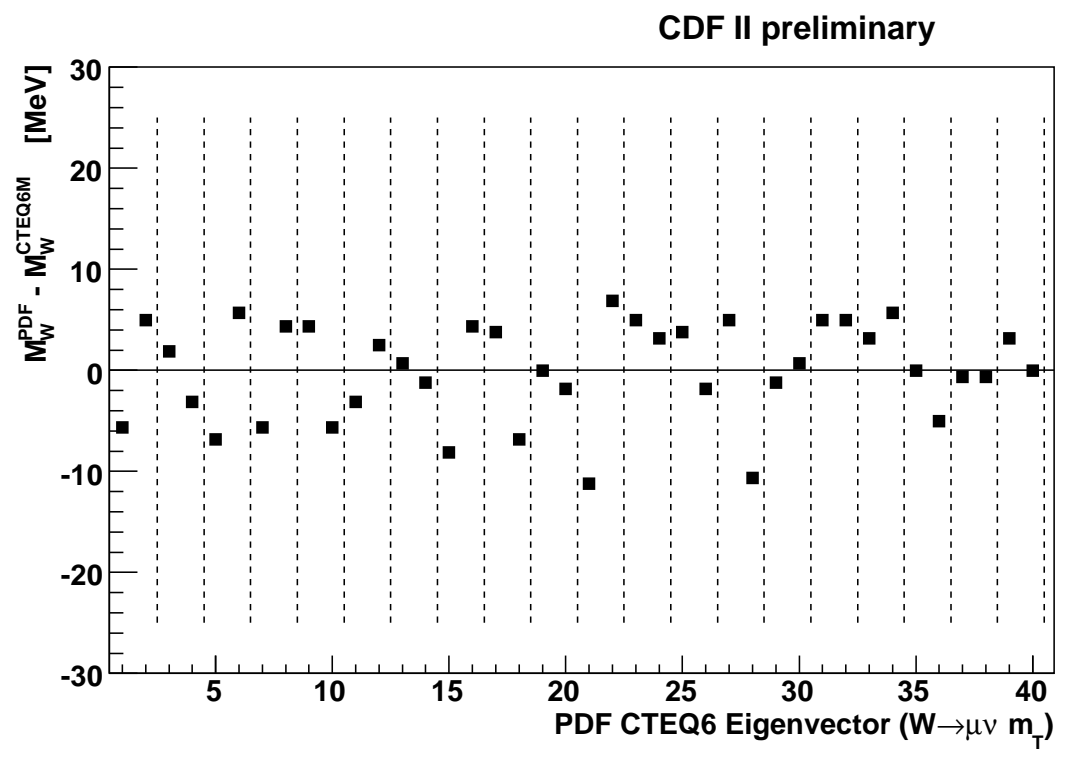

Figure 6.1: Variations of fitted $m_{W}$ difference from the default using $m_{T}$ distributions over 20 pairs of CTEQ6 error PDFs. The difference in $M_{W}$ is between each error PDF and the default CTEQ6M.

The following formula is provided by the CTEQ6 Collaboration to compute the uncertainty associated with the $90 \%$ CL:

$$
\left(\Delta M_{W}\right)_{90 \% \mathrm{CL}}=\frac{1}{2} \sqrt{\sum_{i=1}^{N=20}\left(\Delta M_{W}\left(S_{i}^{+}\right)-\Delta M_{W}\left(S_{i}^{-}\right)\right)^{2}}
$$

where $S_{i}$ represents the 20 pairs of the PDF eigenvectors and $\Delta M_{W}\left(S_{i}^{+}\right)\left(\Delta M_{W}\left(S_{i}^{-}\right)\right)$ corresponds to shift in the fitted value due to positive (negative) variation of eigenvector $S_{i}$. Since both variations are computed, a factor of $1 / 2$ is introduced to account for double-counting. The $90 \% \mathrm{CL}$ systematic uncertainty on $m_{W}$ in $W \rightarrow \mu \nu$ channel is found to be $20,21,22 \mathrm{MeV}$ on $m_{T}, p_{T}$ and $p_{T}^{\prime}$ fits, respectively. Applying the same 


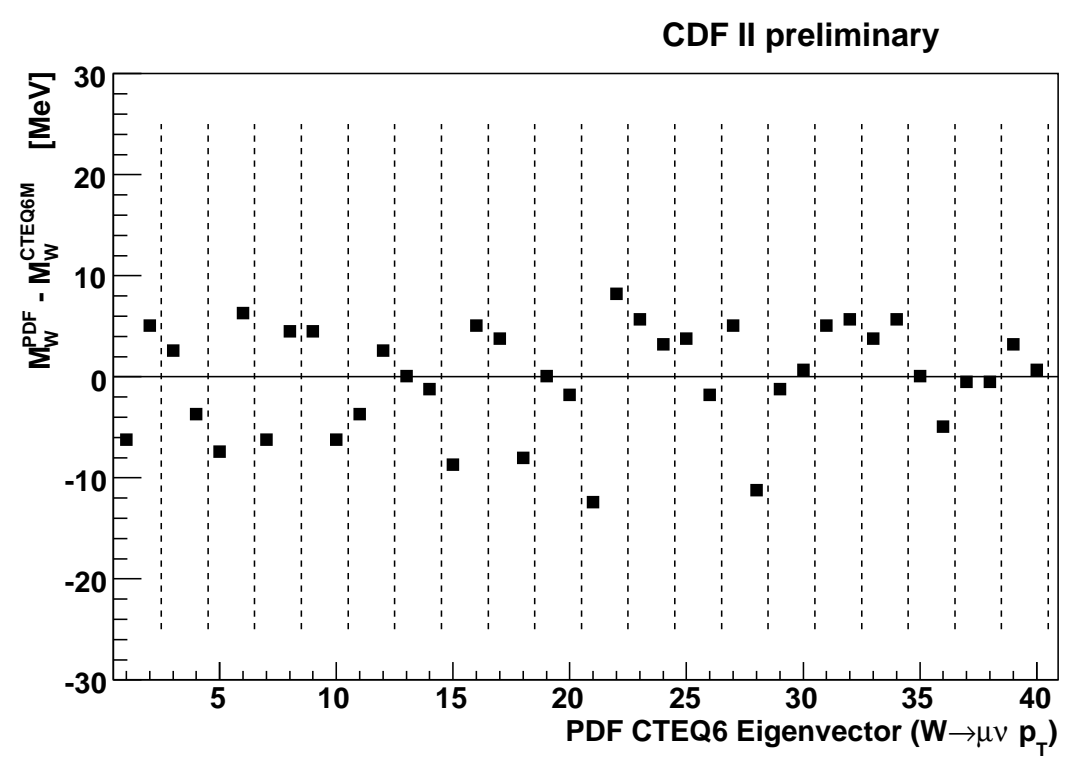

FIGURE 6.2: Variations of fitted $m_{W}$ difference from the default using $p_{T}^{\mu}$ distributions over 20 pairs of CTEQ6 error PDFs. The difference in $M_{W}$ is between each error PDF and the default CTEQ6M.

method to $W \rightarrow e \nu$ channel, we find the resulting 90\% CL systematic uncertainty to be $19,21,21 \mathrm{MeV}$ on $m_{T}, p_{T}$ and $p_{T}^{\nu}$ fits. We convert the $90 \% \mathrm{CL}$ to the more standard $68 \%$ CL $(1 \sigma)$ by dividing by $2.15[53]$ to get $(10,10,10) \mathrm{MeV}$ and $(9,10$, 10) $\mathrm{MeV}$ for $\left(m_{T}, p_{T}, p_{T}^{\nu}\right)$ in the muon channel and electron channel, respectively. A similar but more comprehensive study using MSTW 2008 and CTEQ6.6 has been done by the $W$ mass group [53]. There MSTW 2008 is used as the default for evaluating PDF uncertainties since it supplies a direct formula for $68 \% \mathrm{CL}$. The final PDF uncertainties on $m_{W}$ are quoted to be 10, 9, $11 \mathrm{MeV}$ for $m_{T}, p_{T}$ and $p_{T}^{\nu}$ [53], for both muon channel and electron channel.

\section{2 $W$ and $Z$ Boson $p_{T}$ Model}

The $W$ boson $p_{T}$ is produced by gluon-involved QCD processes such as those shown in Figure 6.4. Since the $p_{T}$ spectra of $W$ and $Z$ bosons at Tevatron peak at only a 


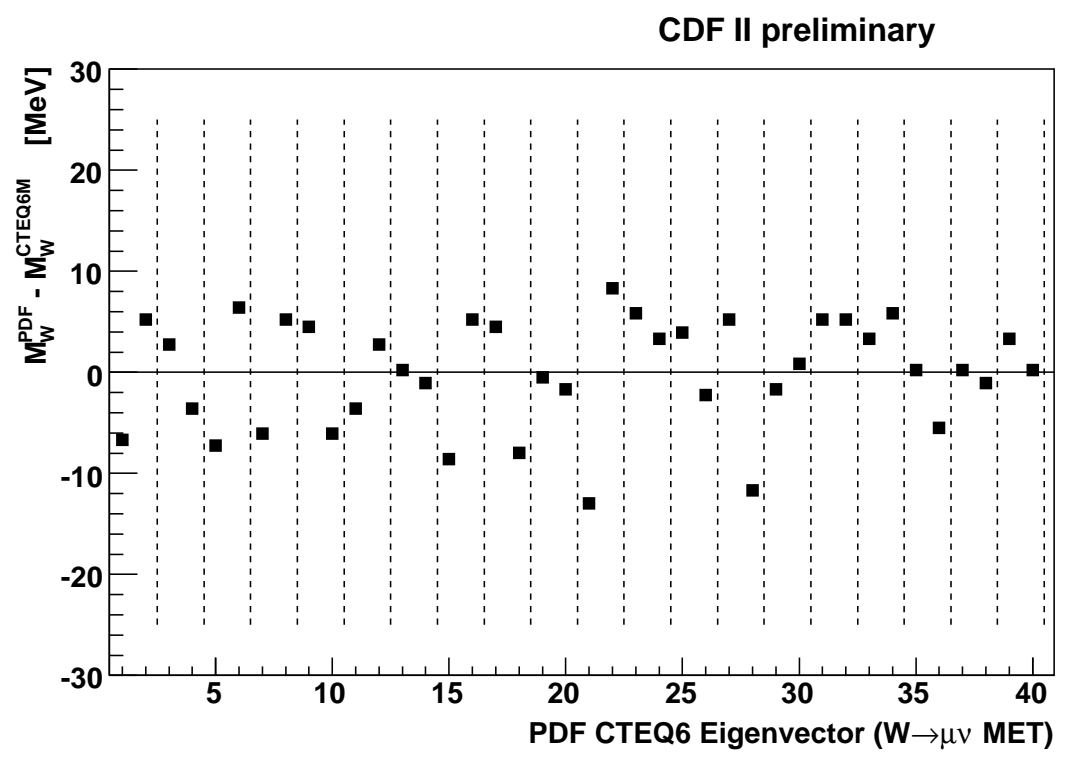

FiguRE 6.3: Variations of fitted $m_{W}$ difference from the default using $p_{T}^{\nu}$ distributions over 20 pairs of CTEQ6 error PDFs. The difference in $M_{W}$ is between each error PDF and the default CTEQ6M.
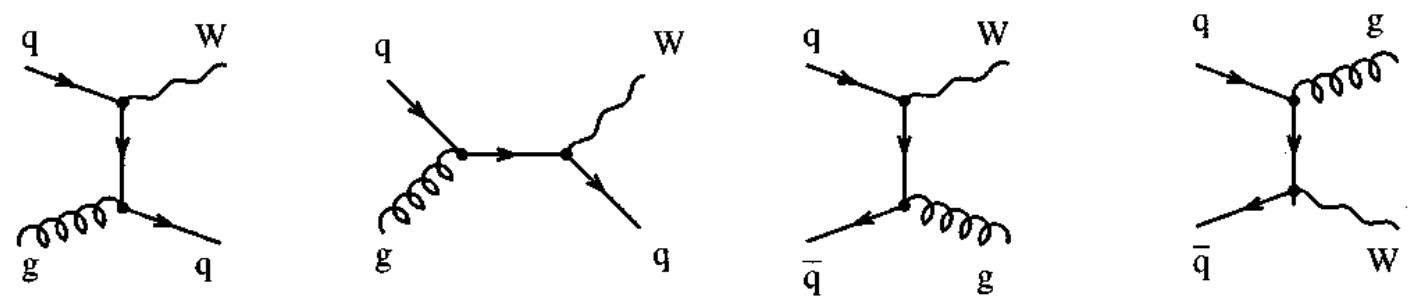

FIGURE 6.4: Leading order QCD processes contributing to the production of $W$ boson. The first diagram is for quark-gluon fusion, the second diagram is for gluon initial state radiation, the rest two diagrams are for associated $W$-gluon production [45]. 
few $\mathrm{GeV}$ and RESBOS gives better description than other generators at low $p_{T}$ region by using a Collins-Soper-Sterman (CSS) [55] multiple soft and collinear gluon resummation, we use RESBOS generator to model boson $p_{T}$ distribution. The CSS frame is a special $W$ boson rest-frame which is constructed to transform the $p_{T}^{W}$ impact onto the $W$ decay angular distribution. The resummation technique reformulates the expansion of perturbative QCD and extends the applicability into low $p_{T}^{W}$ regions with the transition happens in the $p_{T}^{W}$ region between $30 \mathrm{GeV}$ and $50 \mathrm{GeV}$. When $p_{T}^{W}$ values are very low (below $\sim 10 \mathrm{GeV}$ ), the perturbative expansion becomes invalid and non-perturbative description takes over. In RESBOS, the non-perturbative component in calculating the partonic cross section can be parameterized in terms of a Brock-Landry-Nadolsky-Yuan (BLNY) form of the non-perturbative function $\tilde{W}^{\mathrm{NP}}[56]:$

$$
\tilde{W}^{\mathrm{NP}}\left(b, Q, Q_{0}, x_{p}, x_{\bar{p}}\right)=e^{-\left[g_{1}+g_{2} \ln \left(\frac{Q}{2 Q_{0}}\right)+g_{1} g_{3} \ln \left(100 x_{p} x_{\bar{p}}\right)\right] b^{2}}
$$

where $Q_{0}$ is a fixed cutoff at $1.6 \mathrm{GeV}, x_{p}$ and $x_{\bar{p}}$ are the momentum fractions carried by the colliding partons, $b$ is the relative impact parameter of the colliding partons. The three parameters $g_{1}, g_{2}$ and $g_{3}$ are chosen conventionally and are invariant in all processes where initial state includes quarks and final states are colorless objects [57].

Previous studies [8] have shown that $g_{2}$ and $g_{1}$ are $100 \%$ anti-correlated, and $g_{2}$ dominates the influence on fitted $m_{W}$ by changing the peak location of $p_{T}^{W}$ spectrum. This is illustrated by Figure 6.6. To quantify the associated systematic uncertainties on $m_{W}$ due to non-perturbative parameter $g_{2}$, we first fit $g_{2}$ by using $p_{T}^{Z}$ spectrum using $Z \rightarrow \mu \mu$ and $Z \rightarrow$ ee data (see Figure 6.7 for the comparison of dilepton $p_{T}$ spectrum between data and $\mathrm{MC}$ using $Z \rightarrow \mu \mu$ events). The fitted $g_{2}^{C D F}=$ $0.649 \pm 0.013_{\text {stat }}[93]$ for $\alpha_{s}$ fixed at 0.120 shows consistency with the global-fit value $g_{2}^{\text {global }}=0.68_{-0.02}^{+0.01}[56]$. We find a variation of $g_{3}$ by its global-fit uncertainty \pm 0.30 is equivalent to a variation of $g_{2}$ by \pm 0.007 . We thus propagate $g_{3}$ uncertainty into 
$g_{2}$ and get a combined effective $g_{2}$ uncertainty to be 0.015 . This translates into a $W$ boson mass uncertainty of $1 \mathrm{MeV}, 3 \mathrm{MeV}$ and $2 \mathrm{MeV}$ for $m_{T}, p_{T}^{l}$ and $p_{T}^{\nu}$ fits.

The dilepton $p_{T}$ spectrum at intermediate and high $p_{T}$ region is sensitive to $\alpha_{s}$. We find the resulting uncertainty on $m_{W}$ due to a variation of $\alpha_{s}$ to be $2.8 \mathrm{MeV}, 8.0$ $\mathrm{MeV}$ and 3.6 MeV for $m_{T}, p_{T}^{l}$ and $p_{T}^{\nu}$ fits [58].

The uncertainties due to $g_{2}$ and $\alpha_{s}$ are anti-correlated with a correlation coefficient of -0.7 [58]. The $g_{2}$ and $\alpha_{s}$ uncertainties on $m_{W}$ we just obtained are actually $\sigma_{\text {inner }}$ in Figure 6.5, which underestimate the true uncertainties $\sigma_{i}$ when $g_{2}$ and $\alpha_{s}$ are correlated. To combine $g_{2}$ and $\alpha_{s}$ uncertainties on $m_{W}$ by using error propagation, we need to use the uncertainty $\sigma_{i}$ from the simultaneous 2D fit of $g_{2}$ and $\alpha_{s}$, which is related to what we have obtained by $\sigma_{\text {inner }}=\sqrt{1-\rho^{2}} \sigma_{i}$ [32]. Combining $g_{2}$ and $\alpha_{s}$ uncertainties on $m_{W}$ with this correction taken into account, we get a total $p_{T}(W)$ spectrum uncertainty to be $3 \mathrm{MeV}, 9 \mathrm{MeV}$ and $4 \mathrm{MeV}$ for $m_{T}, p_{T}^{l}$ and $p_{T}^{\nu}$ fits (see Appendix $\mathrm{K}$ for details). This is summarized in Table 6.1.

Table 6.1: Systematic uncertainty on $m_{W}$ for $m_{T}, p_{T}$ and $p_{T}^{\prime}$ fits due to $p_{T}(W)$ model $\left(g_{2}, g_{3}\right.$ and $\left.\alpha_{s}\right)$.

\begin{tabular}{lc}
\hline \hline \multicolumn{3}{l}{$\delta m_{W}$ due to } & $p_{T}(W)$ model \\
\hline Distribution & $\delta m_{W}$ \\
\hline$m_{T}(\mu, \nu)$ & $3 \mathrm{MeV}$ \\
$p_{T}(\mu)$ & $9 \mathrm{MeV}$ \\
$p_{T}(\nu)$ & $4 \mathrm{MeV}$ \\
\hline \hline
\end{tabular}

\subsection{W Boson Decay Model}

RESBOS describes the angular component of the differential $W^{ \pm}$boson production 


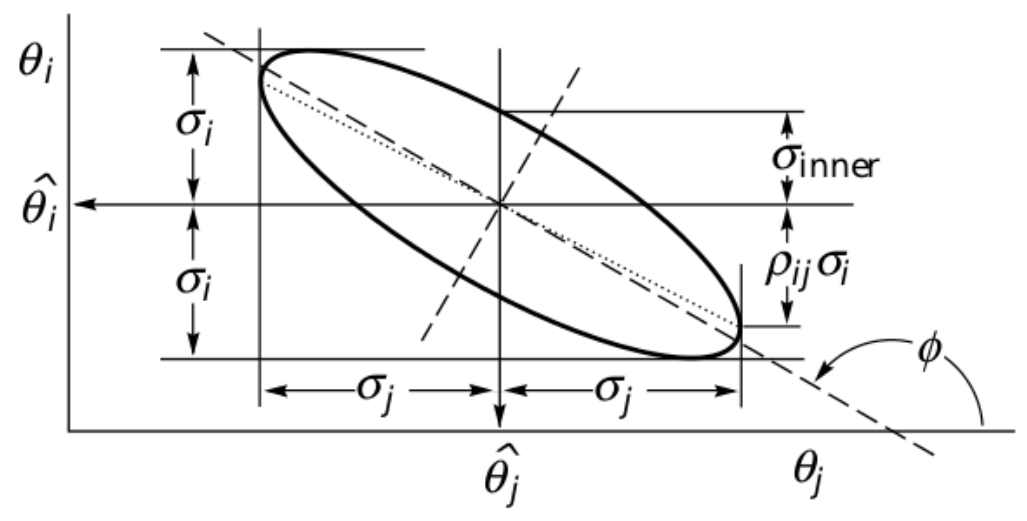

FiguRE 6.5: Standard error ellipse for estimator $\hat{\theta}_{i}$ and $\hat{\theta}_{j}$ which are anti-correlated [32].

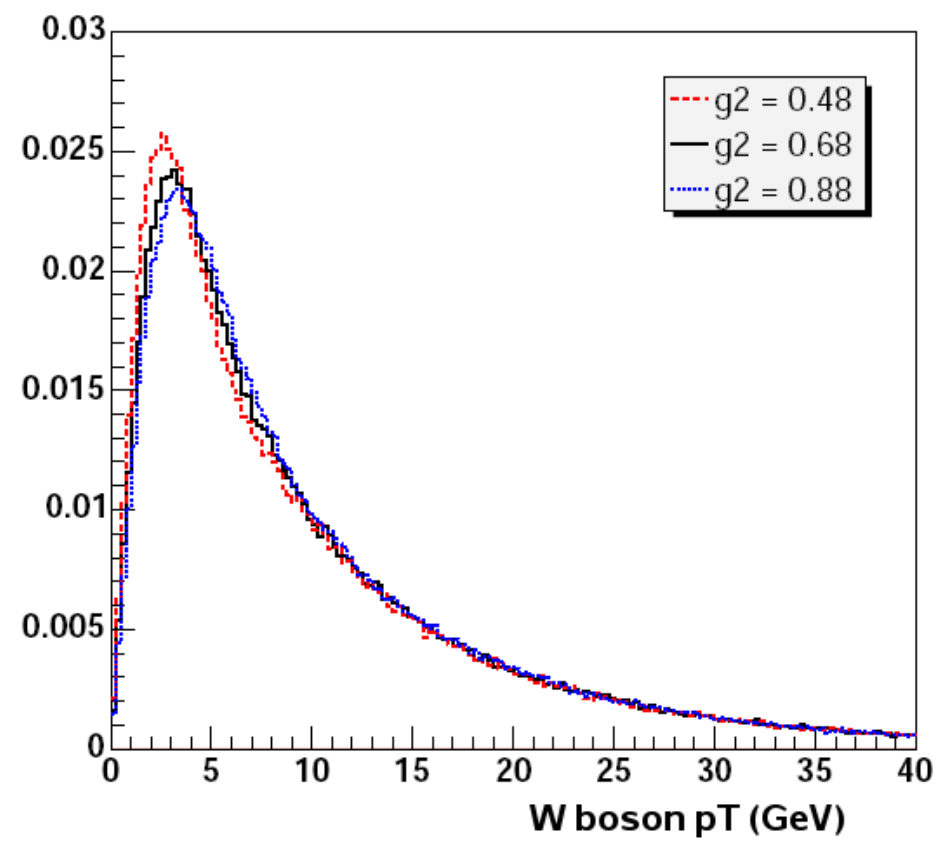

FiguRE 6.6: RESBOS-generated $W$ boson $p_{T}$ spectrum for $g_{2}=0.48 \mathrm{GeV}^{2}, 0.68$ $\mathrm{GeV}^{2}$ and $0.88 \mathrm{GeV}^{2}$. 


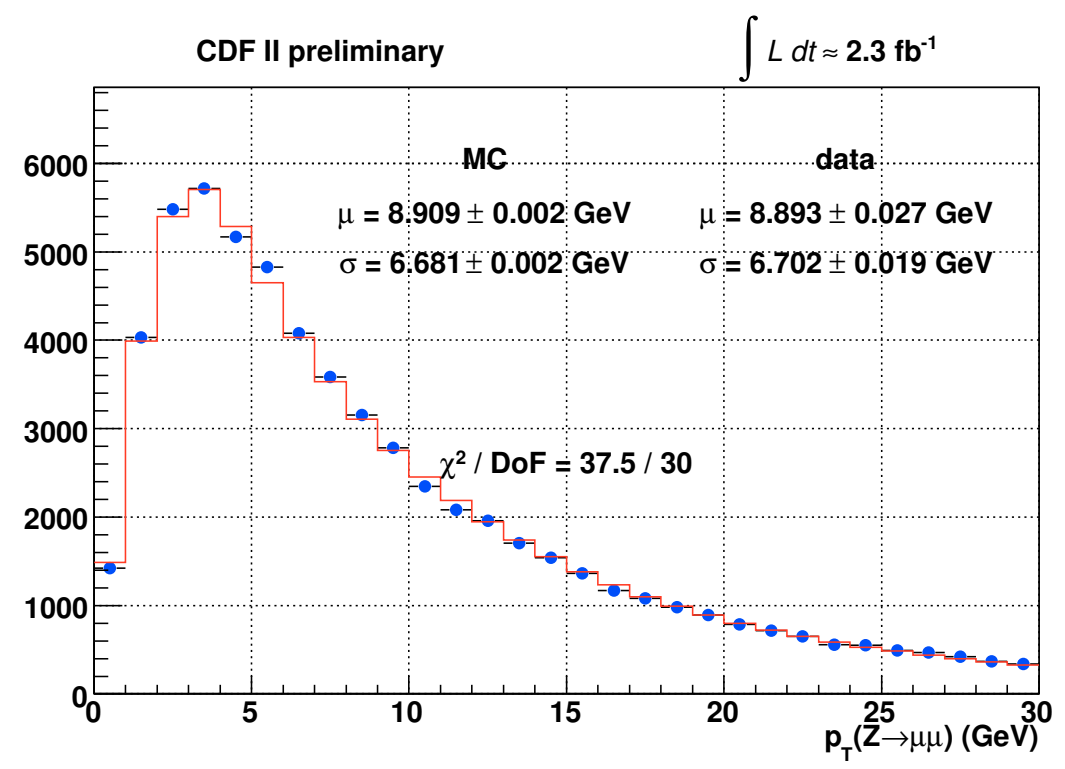

Figure 6.7 : $Z$ boson $p_{T}$ spectrum using $Z \rightarrow \mu \mu$ events. This spectrum, together with the $Z$ boson $p_{T}$ spectrum using $Z \rightarrow e e$ events, is used to fit for $g_{2}$.

cross-section according to the following formula:

$$
\begin{aligned}
\frac{d \sigma}{d \Omega} & \propto\left(1+\cos ^{2} \theta\right)+\frac{1}{2} A_{0}\left(1-3 \cos ^{2} \theta\right) \mp A_{1} \sin 2 \theta \cos \phi \\
& +\frac{1}{2} A_{2} \sin ^{2} \theta \cos 2 \phi+A_{3} \sin \theta \cos \phi \mp A_{4} \cos \theta \\
& +A_{5} \sin ^{2} \theta \sin 2 \phi \mp A_{6} \sin 2 \theta \sin \phi+A_{7} \sin \theta \sin \phi
\end{aligned}
$$

where the angular parameters $\theta$ and $\phi$ are defined in the CSS frame, representing polar and azimuthal angles of the charged lepton, respectively. The helicity amplitudes $A_{i}(i=0, \cdots, 7)$ are functions of $W$ boson $p_{T}$ and are influenced by $W$ boson polarization (or rapidity $y_{W}$ ). Due to the $\mathrm{V}$-A nature of the $W$ boson decay, all $A_{i}$ except $A_{4}$ become zero when the $W$ boson is produced with no transverse motion. The coefficient $A_{4}$ is found to be less than or equal to 2 . It equals 2 when the $W$ boson is produced via valence quark annihilation. When two sea-quarks are involved in the production, $A_{4}$ is 0 .

Studies have been carried to compare $A_{i}$ from RESBos and $A_{i}$ from another 
QCD event generator DYRAD as a function of $p_{T}(W)$ [59]. It is found that both generators agree very well in high- $p_{T}(W)$ region but have noticeable difference in low- $p_{T}(W)$ region. The coefficients $A_{i}$ from RESBOs make a smooth transition to the expected behavior as $p_{T}(W) \rightarrow 0$, since RESBOS uses QCD resummation technique for low $p_{T}(W)$ region. In comparison, DYRAD performs fixed order calculation of $A_{i}$ and is thus different from RESBOs at low $p_{T}(W)$. Reweighting the RESBOs events such that the $A_{i}$ from RESBOs match the values from DYRAD at $p_{T}(W)=25 \mathrm{GeV}$ leads to $3 \mathrm{MeV}$ change in the fitted $m_{W}$. This $3 \mathrm{MeV}$ shift quantifies the impact of the breakdown of fixed-order QCD calculation DYRAD. Since RESBOs supplies a significantly improved description of $A_{i}$ at low- $p_{T}(W)$, the uncertainty in the decay angular distribution in RESBOS is negligible.

\subsection{Photon Radiation}

Three QED processes contribute to the real photon radiation in the event $p \bar{p} \rightarrow W \rightarrow$ $\mu \nu$. They are initial state radiation (ISR) - radiation off the incoming quarks, radiation off the propagator and finial state radiation (FSR) - radiation off the charged lepton. In all QED processes, the most significant effect on $m_{W}$ is from the final state photon radiation off the charged lepton, which shifts the events from the peak region of $m_{T}, p_{T}$ and $p_{T}$ spectra to lower values.

In previous $W$ mass analysis at CDF, we added WGRAD photons to the simulated two-body RESBOS events. WGRAD calculates $O(\alpha)$ electroweak physics including one real final state photon [60]. This effect was captured by using a two dimensional histogram in terms of $\sqrt[3]{y_{\gamma}}$ and $\sqrt{\Delta R(l, \gamma)}$, where $y_{\gamma}=\frac{E_{\gamma}}{E_{\mu}+E_{\gamma}}$ and $\Delta R(l, \gamma)=$ $\sqrt{\left(\Delta \eta_{l \gamma}\right)^{2}+\left(\Delta \phi_{l \gamma}\right)^{2}}$. The cube root form of radiated photon energy fraction $y_{\gamma}$ and square root form of the cone between muon and photon $\Delta R(l, \gamma)$ are chosen to transform the sharp distributions of $y_{\gamma}$ and $\Delta R$ for ease of Monte Carlo sampling in 
our fast simulation.

At the early stage of this round $W$ mass measurement, we sampled the $2-\mathrm{d} \sqrt[3]{y_{\gamma^{-}}}$ vs- $\sqrt{\Delta R(l, \gamma)}$ histogram for the second time to study the effects on $m_{W}$ due to the second photon radiation off the charged lepton in the $W$ leptonic decays, assuming the second photon has similar energy and angular distributions as the first photon and $\geq 3$ photon effects are negligible for the precision we want to reach. This second photon is required to have less energy than the first radiated photon and is only simulated when the energy of the first photon is above some threshold $(\sim$ a few MeV). Theoretical calculations [61] suggest the shift in $m_{W}$ due to higher-order photon radiation is $\sim 10 \mathrm{MeV}$ for both electron channel and muon channle. We observed $11 \mathrm{MeV}$ shift in the electron channel but however only $\sim 3 \mathrm{MeV}$ shift in the muon channel using our method of sampling the WGRAD 2-d histogram twice.

We treat the QED part more thoroughly by feeding RESBOS-generated events into PHOTOS, which has a better description of the relevant QED processes including final state multi-photon radiation with the appropriate rates, energy and angular distributions. The FSR-only PHOTOS program is based on an exact multiphoton phase space with iterative algorithm to simulate QED effects in decay of intermediate particles and resonances [62] [63]. It adds extra photons with certain probability and updates the kinematics of involved particles. In order to validate the result obtained from PHOTOs program, we use another package called HORACE [64] [65] for comparison. Two versions of the HORACE program are available for this cross-check, which we call OLD HORACE and NEW HORACE. The OLD HORACE is similar to the PHotos program, which implements multi-photon FSR only. Detailed studies show that the QED generators OLD HORACE and PHOTOS agree with each other quite closely in the photon rates and distributions [66]. This is illustrated in Figure 6.8. The difference between FSR-only OLD HORACE and FSR-only PHOTOS is found to be 
-7 MeV on $m_{W}$ in the electron channel and $0 \mathrm{MeV}$ on $m_{W}$ in the muon channel, with an uncertainty of $3 \mathrm{MeV}$ from MC statistics [66]. We also compare in detail the FSRonly OLD HORACE and NEW HORACE, which is the best available event generator to date describing physical effects including ISR, FSR, ISR-FSR interference, soft and virtual corrections. The comparison shows we need to correct $m_{W}$ in both the electron and the muon channel by $+4 \mathrm{MeV}$, with an uncertainty of $2 \mathrm{MeV}$ [67]. The combined effect of the two corrections suggest that we need to correct the final $m_{W}$ central value by $-3 \mathrm{MeV}$ in the electron channel and $+4 \mathrm{MeV}$ in the muon channel as calibration corrections from PHOTOS to the NEW HORACE calculation, since we use Pнотоs as the default event generator for $m_{W}$ measurement [93] .

The final QED systematic uncertainties on $m_{W}$ comes from several components. We take the $3 \mathrm{MeV}$ MC statistical uncertainty of FSR-only OLD HORACE and FSRonly PHOTOS comparison as an systematic uncertainty to cover any generator dependence. The $2 \mathrm{MeV}$ uncertainty from OLD HORACE and NEW HORACE comparison covers ISR and ISR-FSR interference which are neglected by PнотOS. Studies of the soft and virtual correction in NEW HORACE suggest the uncertainty in this correction could be up to $1 \mathrm{MeV}$ [67]. We vary the minimum photon radiation cutoff from 0.4 $\mathrm{MeV}$ to $4 \mathrm{MeV}$ and find the change on $m_{W}$ is $2 \mathrm{MeV}$. The uncertainty in $\gamma \rightarrow e e$ splitting process is estimated to be $1 \mathrm{MeV}$. The uncertainty due to hard-photon modelling is found to be $0.3 \mathrm{MeV}$ and thus negligible. The total QED uncertainty on $m_{W}$ is $4 \mathrm{MeV}$ for both the electron and muon channel.

Table 6.2 summarizes the systematic uncertainties on $m_{W}$ arising from QED/Photon radiation processes. The effect of QED and QCD interference is negligible (at most $\sim 1 \%)[68]$. 

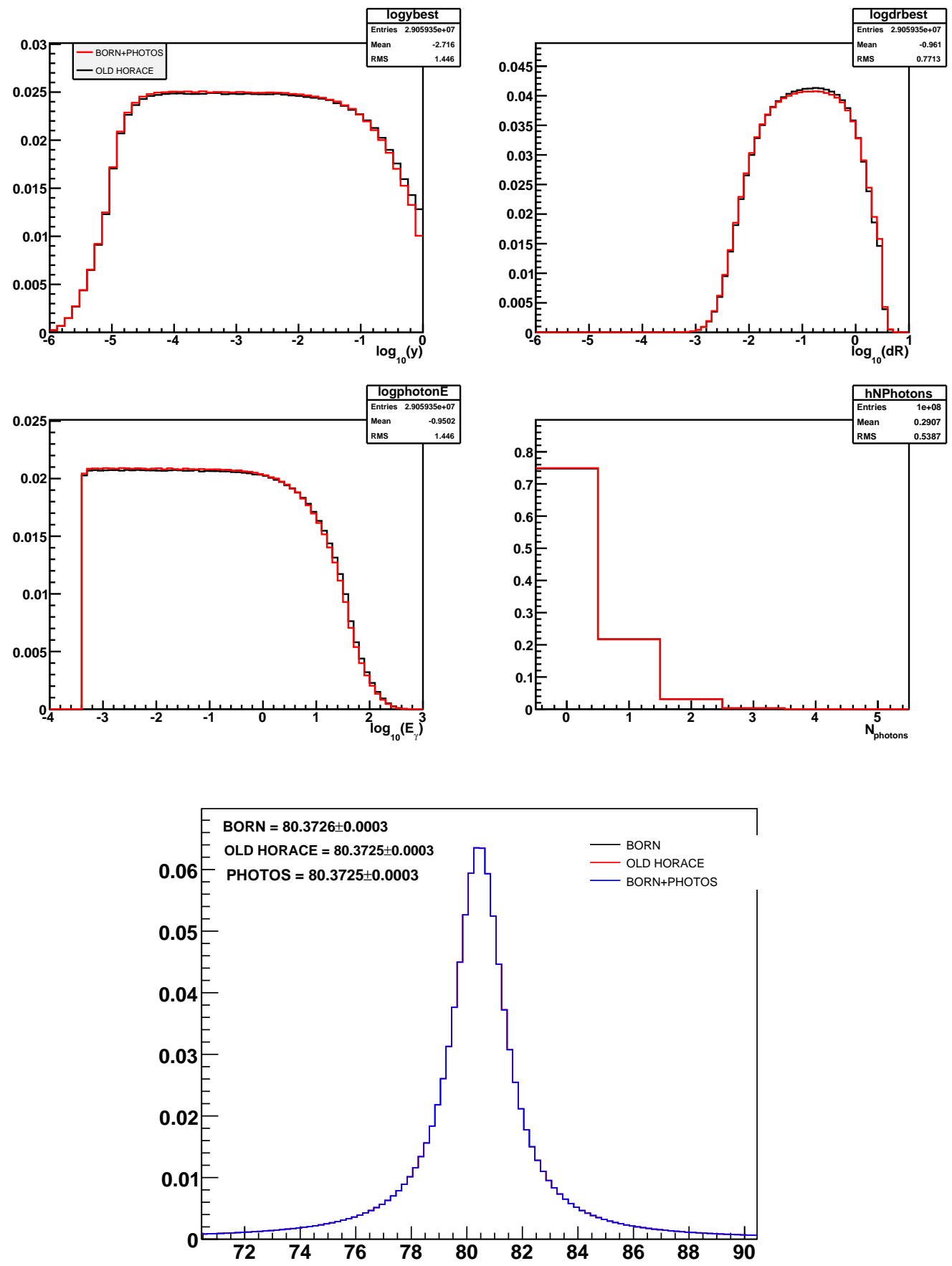

FiguRE 6.8: The four plots on the top show, for the $W^{+} \rightarrow \mu^{+} \nu+n \gamma$ process, the comparison of $\log _{10}\left(y_{\gamma}\right), \log _{10}(\Delta R(l \gamma)), \log _{10}\left(E_{\gamma} / \mathrm{GeV}\right)$ and $n_{\gamma}$, respectively. The $\Delta R$ is computed with respect to the muon. The bottom plot shows the comparison of invariant mass of all final state particles using OLD HORACE and PHOTOS. [66] 
Table 6.2: Systematic uncertainty on $m_{W}$ for $m_{T}, p_{T}$ and $p_{T}$ fits due to photon radiation.

\begin{tabular}{lc}
\hline \hline \multicolumn{3}{l}{$\delta m_{W}$ due to photon } & radiation \\
\hline Distribution & $\delta m_{W}(\mathrm{MeV})$ \\
\hline$m_{T}$ & 4 \\
$p_{T}^{\mu}$ & 4 \\
$p_{T}^{\nu}$ & 4 \\
\hline \hline
\end{tabular}




\section{7}

\section{Fast Detector Simulation}

To study a wide range of systematic sources for the $W$ mass measurement, we need a tunable, detailed and fast detector simulation. Since the standard CDF simulation (cdfSim) is CPU-intensive and not sufficiently tunable, a custom fast detector simulation (DukeSim) is developed using a parameterized detector model to simulate the detector response to muons, electrons, photons and the hadronic recoil [69]. DukeSim is several orders of magnitude faster than cdfSim while capturing the details of relevant physics. It thus has the flexibility to quantify the influences of various inputs with a speed fast enough for frequent high-statistics studies. DukeSim works by reading in the ASCII file of momentum 3-vectors of the two-body decay products from the resonance of study $(W, Z, J / \psi, \Upsilon)$. These 3 -vectors can be obtained from any generator which provides all the generator-level physics. It will then update the event kinematics by simulating all relevant detector effects. Currently, DukeSim can simulate $W \rightarrow \mu \nu, Z \rightarrow \mu \mu, W \rightarrow e \nu, Z \rightarrow e e, J / \psi \rightarrow \mu \mu$ and $\Upsilon \rightarrow \mu \mu$ events. For $W$ bosons, a series of templates are produced by a linear interpolation between two templates, which are generated in the fast simulation by processing vector files with input $m_{W}=80 \mathrm{GeV}$ and $m_{W}=81 \mathrm{GeV}$. Similar procedure is adopted for $Z$ boson 
templates using input vector files generated at $m_{Z}=90.687 \mathrm{GeV}$ and $m_{Z}=91.687$ $\mathrm{GeV}$.

In this chapter, we will describe the modelling of the COT response and resolution, muon acceptance and muon efficiency. The calorimeter response and resolution to the hadronic recoil are parameterized by a number of tunable parameters and will be discussed in detail in Chapter 10.

\subsection{Ionization Energy Loss}

The simulation of muon energy inside the CDF detector is crucial for a precise $m_{W}$ measurement. The muons lose energy mainly through ionization in the COT active volume and the material upstream of it (beampipes, SVX and ISL detectors and COT inner wall). The mean rate of energy loss is given by the well-known Bethe-Bloch formula [32]:

$$
-\frac{d E}{d x}=\frac{K}{\beta^{2}}\left[\frac{1}{2} \ln \frac{2 m_{e} \beta^{2} T_{\max }}{\left(1-\beta^{2}\right) I^{2}}-\beta^{2}-\frac{\delta}{2}\right],
$$

where $K$ is an overall constant factor, $\beta$ is the particle velocity, $I$ is the mean excitation energy, $T_{\max }$ is the maximum kinematic energy that can be given to a free electron in a single collision, and $\delta$ is the density effect correction which depends on the material. We take the material to be silicon throughout in the calculation of $\delta$ using Sternheimer's parameterization [70].

A three-dimensional lookup table, known as Silimap, is used to calculate the ionization energy loss in the the material upstream of the COT $(r<40 \mathrm{~cm})$. Silimap is created by scanning the full detector geometry by taking into account the material measurements during construction and during the study of photon to electron conversion. Silimap stores three material properties - the number of radiation length $x_{0}$, the normalization factor $K$ and the ionization potential $I$ - in a finely binned three-dimensional histogram as a function of radius $r$, azimuth $\phi$ and $z$. 
The energy loss model in our fast simulation is scaled by a global correction factor 1.043. The factor is obtained from $J / \psi \rightarrow \mu \mu$ sample by requiring the reconstructed di-muon invariant mass to be independent of the mean inverse momentum of the two decay muons. See Chapter 8 for detailed discussion.

\subsection{Multiple Coulomb Scattering}

In the fast simulation, a charged muon is propagated along a helical trajectory from the production point. While the muon is stepping into the COT, the number of radiation lengths $x_{0}(r, \phi, z)$ which describes the passive material is obtained from the Silimap at each interaction layer. The muon track is then randomly scattered according to the predicted amount of small-angle Coulomb scattering. A Gaussian distribution which well describes the central $98 \%$ of the angular distributions is used in the fast simulation. The width of this central Gaussian is given by [32]:

$$
\sigma_{\theta}=\frac{13.6 \mathrm{MeV}}{\beta \cdot c \cdot p} z \sqrt{x / X_{0}}
$$

where $p$ is the muon momentum in units of $\mathrm{MeV}, \beta \cdot c$ is the muon velocity, $z$ is the

muon charge and $\sqrt{x / X_{0}}$ is the thickness of material in units of radiation lengths. To model the tails of angular distribution observed in MUSCAT data [71], we use a second Gaussian with a width 3.8 times wider to account for the remaining $2 \%$ of the scatters.

\subsection{COT simulation and reconstruction}

Observed worsening of the COT hit resolution in the inner superlayers (SLs) due to the higher occupancy motivates a SL-dependent resolution model in the fast simulation. As shown in Figure 7.1, we obtain the hit resolution in each of the eight SLs by using the residuals in $Z \rightarrow \mu \mu$ data tracks. Table 7.1 summarizes those eight resolution numbers. For mass fits to any given resonance, we introduce a hit resolution 
scale factor $h_{0}$ which multiplies the hit resolutions in Table 7.1. This parameter $h_{0}$ is tuned to match the width of the observed mass peak, and is typically close to unity.

Table 7.1: COT hit resolution for superlayers 1 to 8 .

\begin{tabular}{lcccccccc}
\hline \hline & SL1 & SL2 & SL3 & SL4 & SL5 & SL6 & SL7 & SL8 \\
\hline COT hit resolution $(\mu m)$ & 181 & 158 & 163 & 148 & 150 & 144 & 144 & 142 \\
\hline \hline
\end{tabular}

The five track parameters $d_{0}, \phi_{0}, c, z_{0}$ and $\cot \theta$ are determined from a helix fit to the smeared hits, where $d_{0}$ is the impact parameter of the fitted track with respect to the origin, $\phi_{0}$ is the azimuthal angle at $d_{0}, c \equiv q /(2 R)$ is the curvature of the helix with $q$ the muon charge and $R$ the radius of curvature, $z_{0}$ is the $z$ coordinate at $d_{0}$, and $\cot \theta$ is the cotangent of the polar angle. The particle energy is reduced by the ionization energy loss sampled from the Landau distribution [32]. Depending on the multiple scattering and ionization energy loss experienced by the muon, the helix parameters $c, \phi_{0}$ and $d_{0}$ are updated correspondingly at each interaction. The updated helix is used to propagate the muon to the next layer of material.

The transverse momentum of a given muon can be calculated using the Lorentz equation:

$$
p_{T}=\frac{q B}{2|c|}
$$

where $B$ is the strength of magnetic field. At CDF, $q B / 2$ is found to be $2.11593 \times 10^{-3}$ $\mathrm{GeV} / \mathrm{cm}$. Studies have shown that momentum resolution is related to the number of hit points, the hit resolution, and the projected length of track onto the $r-\phi$ plane by equation [72]:

$$
\frac{\sigma\left(p_{T}\right)}{p_{T}} \propto \frac{p_{T} \sigma}{B L^{2}} \sqrt{\frac{720}{N+5}}
$$



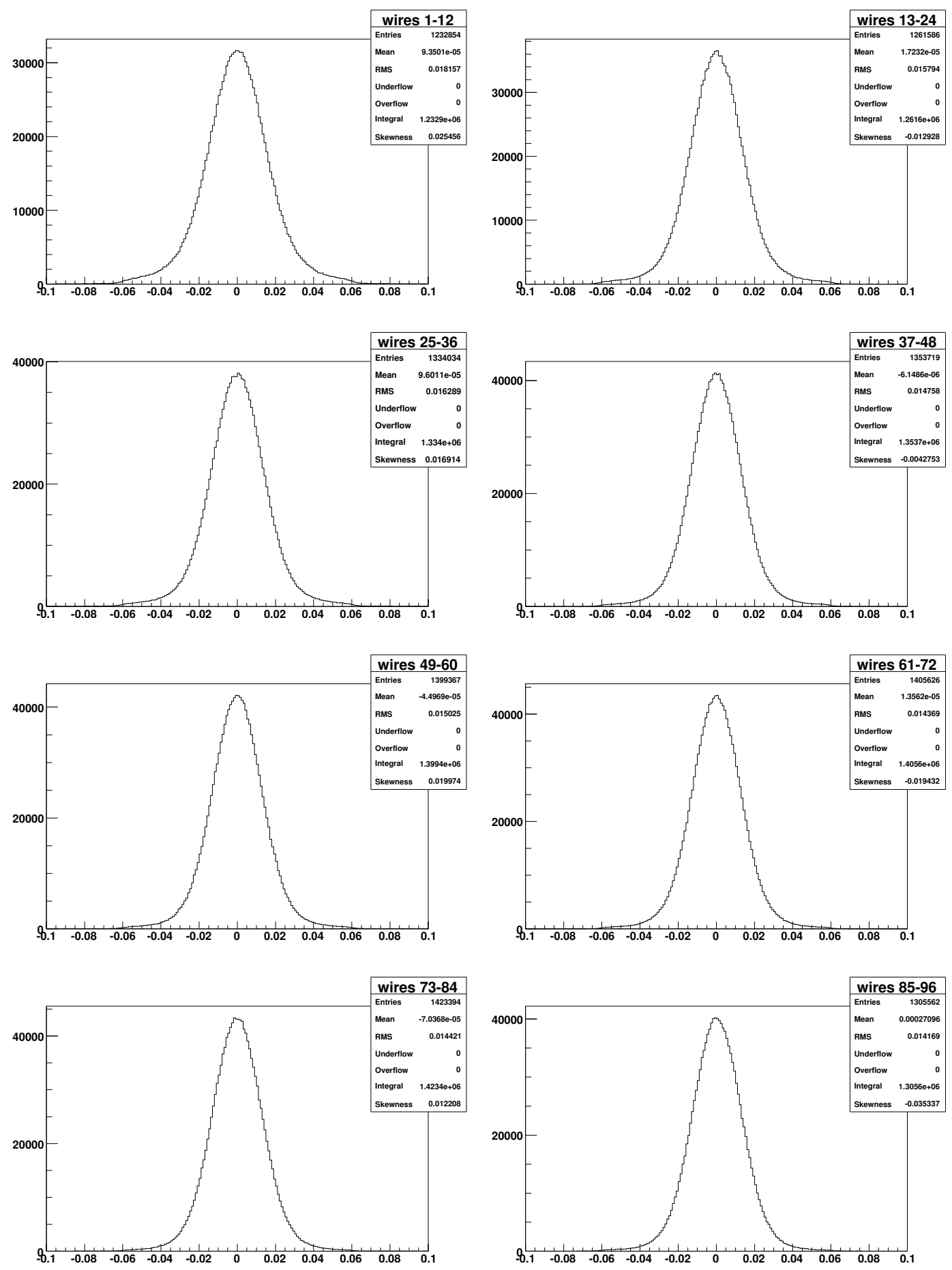

FiguRE 7.1: COT hit residuals for each superlayer, extracted from $Z \rightarrow \mu \mu$ data tracks. 
Increasing the track length $L$, hit points $N$ as well as improving the resolution $\sigma$ (reducing $\sigma$ in value) can thus improve the momentum resolution. We can also see that higher $p_{T}$ tracks have worse momentum resolution since they bend less in the magnetic field. The COT transverse momentum resolution is found to be $\sigma_{p_{T}} / p_{T}=1.7 \times 10^{-3} p_{T} / \mathrm{GeV}[73][74]$ using reconstructed cosmic ray events.

In the fast simulation, we have the option to constrain the helix to originate from the location of the beam. The beamspot size of $(42 \pm 1) \mu m$ is derived by comparing the $Z \rightarrow \mu \mu$ invariant mass line shapes between data and MC. This adds an additional constraint in the helix fit at a distance of at least $40 \mathrm{~cm}$ from the innermost COT hit points, and improves the momentum resolution significantly to $\sigma p_{T} / p_{T} \approx 0.5 \times 10^{-3} p_{T} / \mathrm{GeV}[8]$.

\subsection{Muon Identification Efficiency Modelling}

The event selection requirements can introduce some biases. The dominant bias is found to be the dependence of the muon identification efficiency on the recoil projection in the direction of the muon $\left(u_{\|}\right)$. Figure 7.2 shows such dependence by using $Z \rightarrow \mu \mu$ events with the dependence modelled as a linear function:

$$
\epsilon=A\left[1+B\left(u_{\|}+\left|u_{\|}\right|\right)\right]
$$

where $A$ is an overall efficiency normalization that does not affect the $m_{W}$ measurement, $B$ is a parameter governing the linear dependence on $u_{\|}$. This particular form of efficiency modelling is motivated by the following considerations: when $u_{\|}$is opposite to the muon direction, the identification efficiency of the muon should not be affected by $u_{\|}$; however, as $u_{\|}$increases along the muon direction, there are more recoil energy collinear with the muon to worsen the muon identification. By fitting against data, we find:

$$
B=\left(-0.17 \pm 0.07_{\text {stat }}\right) \times 10^{-3} / \mathrm{GeV}
$$


To estimate the systematic uncertainty on $m_{W}$ due to the muon identification efficiency, we use MC pseudoexperiments and find the $1 \sigma$ uncertainy on $m_{W}$ is $(0,1,2)$ $\mathrm{MeV}$ for $m_{T}, p_{T}^{\mu}$ and $p_{T}^{\nu}$ fits.

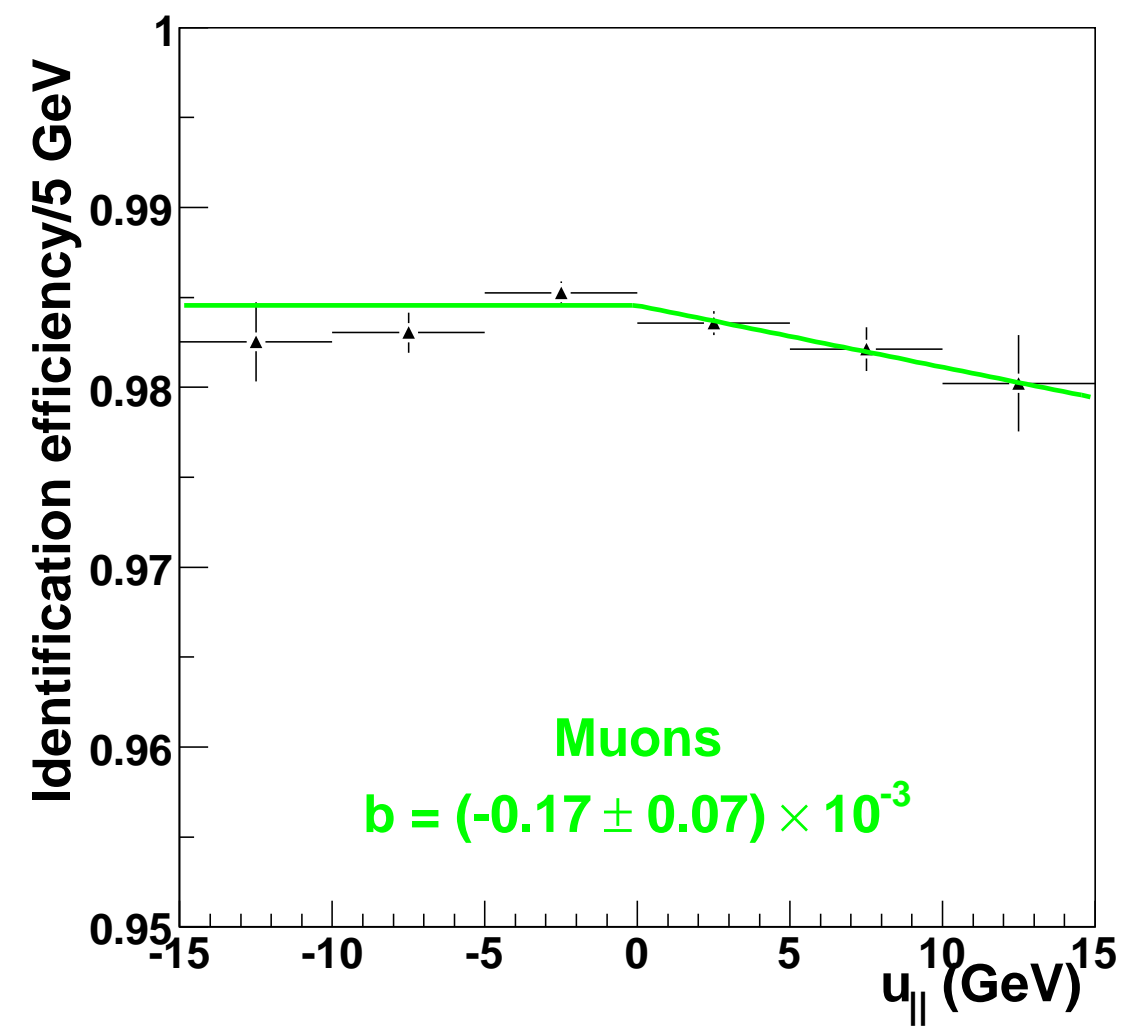

FiguRE 7.2: Variation of the muon identification efficiency as a function of $u_{\|}$, the recoil componenent in the direction of the muon.

\subsection{Muon Acceptance Modelling}

The acceptance of the muon detectors is extracted from the standard CDF simulation (cdfSim) by further requiring the muon to be fully fiducial to the COT with a cut on $z$ of the muon to be $|z|<155 \mathrm{~cm}$. Single muons with a flat distribution in $\phi$ and $\eta$ are generated and propagated through cdfSim to calculate the probability of muon 
identification within the CMUP and CMX detectors as a function of $\phi, \cot \theta$ and $z_{0}$. Since the probability dependence on $z_{0}$ is small and little correlation is observed between $\phi$ and $\cot \theta$, we create one-dimensional efficiency look-up histograms in $\phi$ and $\cot \theta$ for both CMUP and CMX (see Figure 7.3).
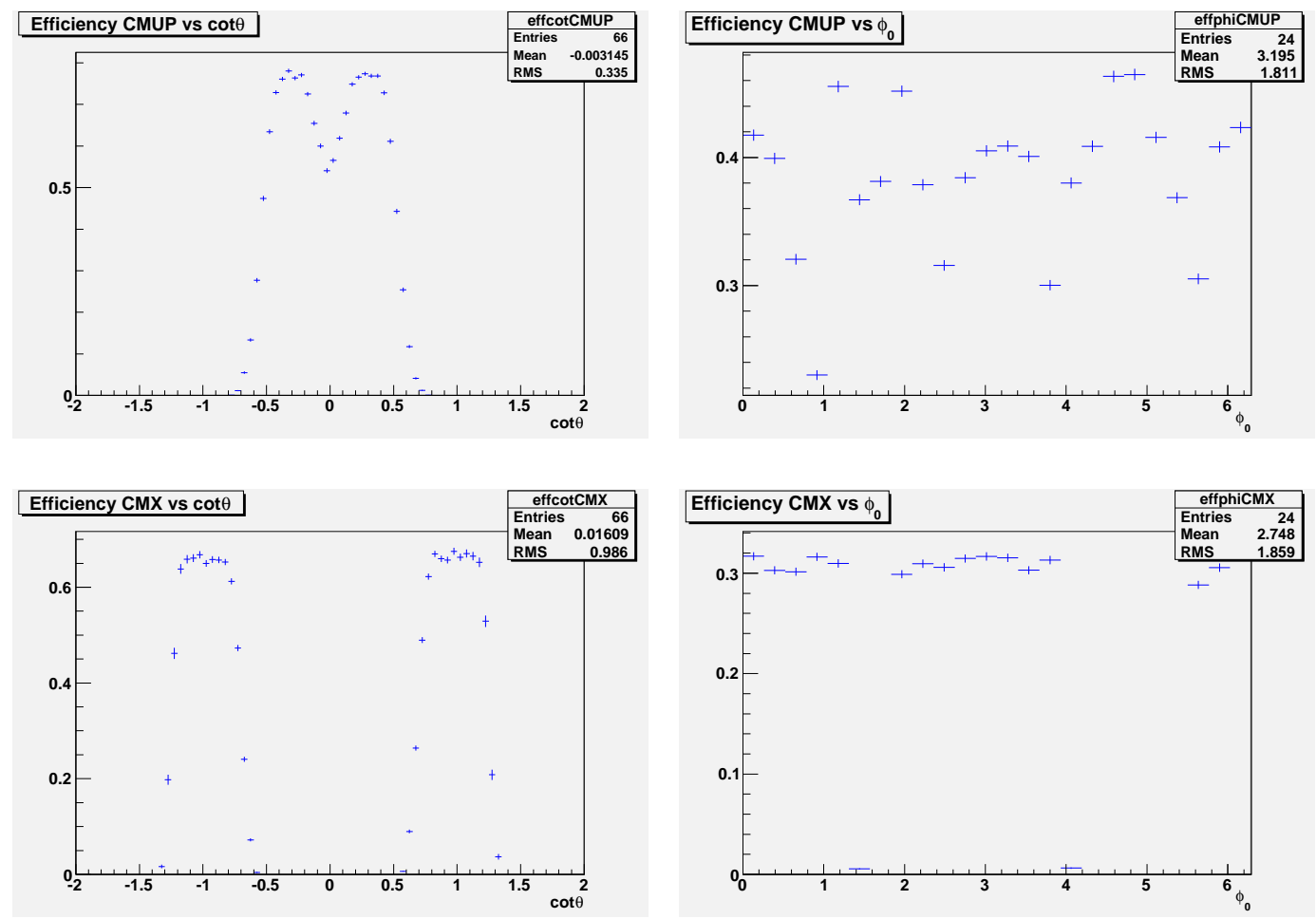

Figure 7.3: Muon acceptance efficiency as a function of $\cot \theta$ (left) and $\phi$ (right). The top (bottom) row is for CMUP (CMX).

To preserve the relative trigger and stub efficiencies for CMUP and CMX observed in $W \rightarrow \mu \nu$ data, we apply a scale factor to the CMUP histograms such that the maximum efficiency is 0.86 [8]. With the four 1-d histograms from CMUP and CMX, we can model the complete muon acceptance probability in the fast simulation as:

$$
\epsilon^{\mu}(\phi, \cot \theta)=\epsilon_{\phi}^{\mathrm{CMUP}}(\phi) \cdot \epsilon_{\cot \theta}^{\mathrm{CMUP}}(\cot \theta)+\epsilon_{\phi}^{\mathrm{CMX}}(\phi) \cdot \epsilon_{\cot \theta}^{\mathrm{CMX}}(\cot \theta) .
$$




\section{8}

\section{Momentum Scale}

The COT momentum scale $(1+\Delta p / p)^{-1} \approx(1-\Delta p / p)^{1}$ is a scale factor applied to the COT-measured momentum to estimate the true momentum. It is the most important quantity for the $W$ boson mass measurement in the muon decay channel since the transverse momentum of muon $\left(p_{T}^{\mu}\right)$ goes directly into the transverse mass formula $m_{T}$. An incorrect momentum measurement will shift the $m_{T}$ distribution from its real distribution, thus bias the measured $m_{W}$ significantly. The COT momentum scale obtained in the muon decay channel also impact the $m_{W}$ measurement in the electron channel, where the momentum scale is transfered to the calorimeter energy scale using the $E / p$ distribution of electrons from $W \rightarrow e \nu$ events. It is thus important to determine the momentum scale precisely such that we can reach the goal of measuring $m_{W}$ with $<25 \mathrm{MeV}$ precision.

We use a large data sample of $J / \psi$ and $\Upsilon(1 S)$ quarkonium resonances to set the COT momentum scale $(1-\Delta p / p)$ by using the fact that their measured worldaveraged masses are precisely known to be $m_{J / \psi}=3096.87 \pm 0.04 \mathrm{MeV} / \mathrm{c}^{2}$ and

\footnotetext{
${ }^{1}$ We define $\Delta p / p=S_{p}-1$ where $S_{p}=\frac{p_{\text {measured }}}{p_{\text {true }}}$, thus we have $p_{\text {true }}=\frac{p_{\text {measured }}}{S_{p}}=p_{\text {measured }}(1+$ $\Delta p / p)^{-1} \approx p_{\text {measured }}(1-\Delta p / p)$.
} 
$m_{\Upsilon(1 S)}=9460.30 \pm 0.26 \mathrm{MeV} / \mathrm{c}^{2}[32]$, respectively. Another important reason to use $J / \psi$ and $\Upsilon$ resonances is that the di-muon invariant mass spectra from $J / \psi \rightarrow$ $\mu \mu$ and $\Upsilon \rightarrow \mu \mu$ decays are fully reconstructable. A binned maximum likelihood fitting method is used to extract the momentum scale by comparing the invariant mass spectrum from data to a series of invariant mass spectra (i.e. templates) from simulation, with the templates generated as a function of $\Delta p / p$. Since $\Delta p / p$ is only different from the momentum scale by a constant term 1, we will use it and the momentum scale interchangeably when no confusion would arise.

Because the cross section of $J / \psi \rightarrow \mu \mu$ decays at Tevatron is large, the resulting large $J / \psi \rightarrow \mu \mu$ data sample thus allows us to explore any left-over data bias which is not corrected by off-line calibration or COT alignment but is significant for the $m_{W}$ measurement. In our $J / \psi$ data sample, a significant fraction of the events come from secondary decays from B mesons, which can travel some distances to outside of the beam radius before decaying into $J / \psi$ and other products. Because of the existence of those events, COT-only tracks without beam-constraining are used for this sample exclusively.

The $\Upsilon \rightarrow \mu \mu$ decay sample, though has smaller statistical power compared with $J / \psi \rightarrow \mu \mu$ decay sample, can be used as a bridge to study the beam-constrained and non-beam-constrained tracking options since $\Upsilon \rightarrow \mu \mu$ events are promptly produced. The $\Upsilon$ resonance, with a mass of the order $10 \mathrm{GeV}$, also acts an intermediate reference point between the 3 times lighter $J / \psi$ resonance and the 9 times heavier $Z$ boson to study the momentum scale as a function of energy since we want to extrapolate the momentum scale obtained from the low mass resonances to the high mass $W$ boson.

In principle other resonances with sharp di-muon invariant mass spectra can also be used for the COT momentum scale measurement. Considering the limited statistical gain in the final combined result, however, we will only use $J / \psi$ and $\Upsilon(1 S)$ resonances to determine the COT momentum scale for the $W$ boson mass analysis. 
In the following sections, we will first give a description of COT alignment. Then momentum scale measurements from $J / \psi$ and $\Upsilon(1 S)$ samples will be presented, see Figure 8.1 for illustration of the analysis procedure. Finally, we obtain a combined momentum scale from $J / \psi$ and $\Upsilon(1 S)$ results.

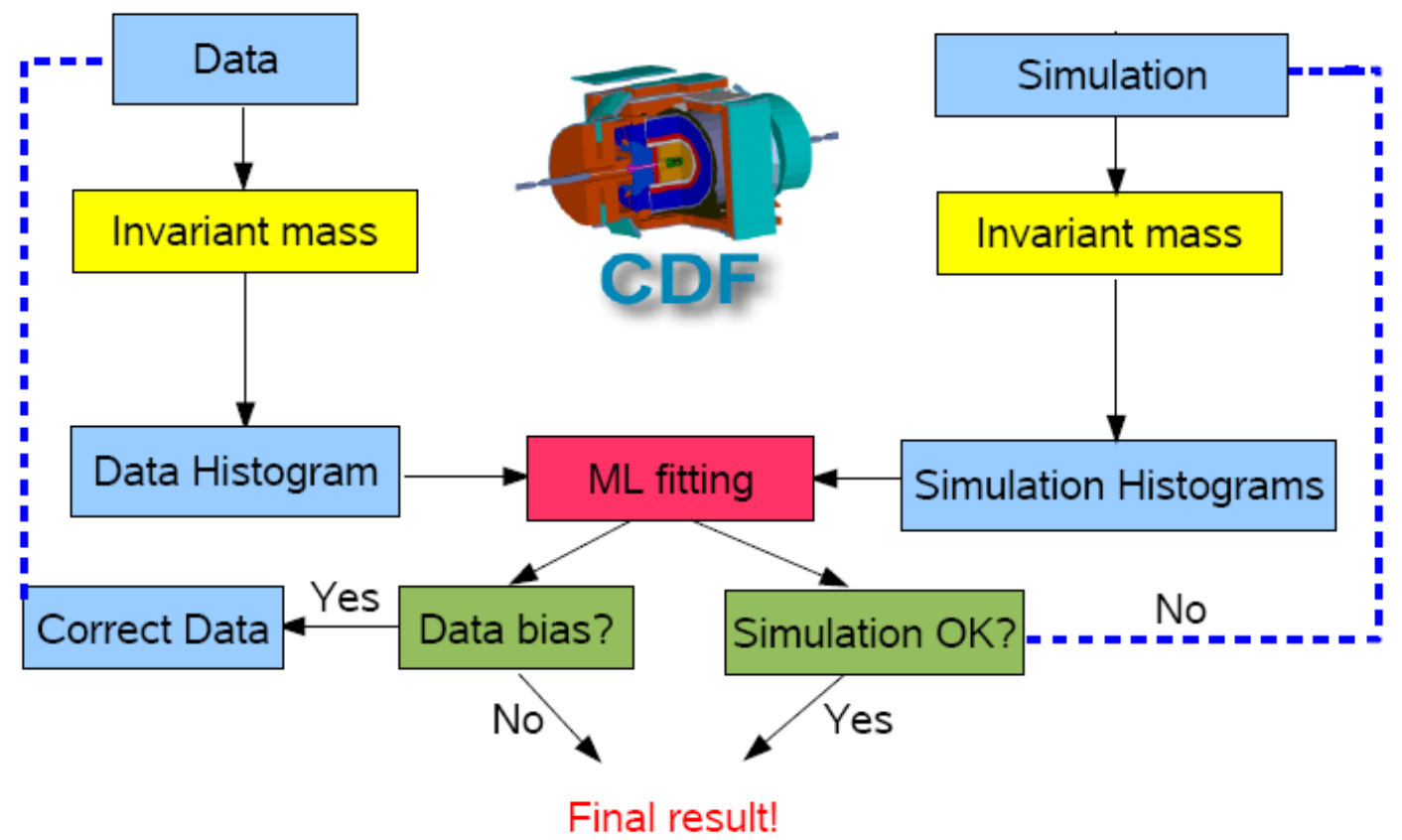

Figure 8.1: Flow chart of momentum scale (or $\Delta p / p$ ) measurement.

\subsection{COT Alignment}

To precisely determine the positions of charged particles passing through the COT, we need to have a precise knowledge of where the COT sense wires are located throughout the tracking chamber. The COT internal alignment using cosmics is a way to fulfill this goal. Figure 8.2 shows a typical cosmic ray event recorded by the CDF detector. We use cosmic ray events from the same data taking period as the $2.2 \mathrm{fb}^{-1} W \rightarrow \mu \nu$ dataset by refitting a single helix to the two tracks which are on opposite sides of the COT, and calculate hit residuals with respect to the fitted 
helix [75]. The residuals are then used to align each cell by a shift along the global azimuth and a tilt around the cell center, as shown in Figure 8.3.

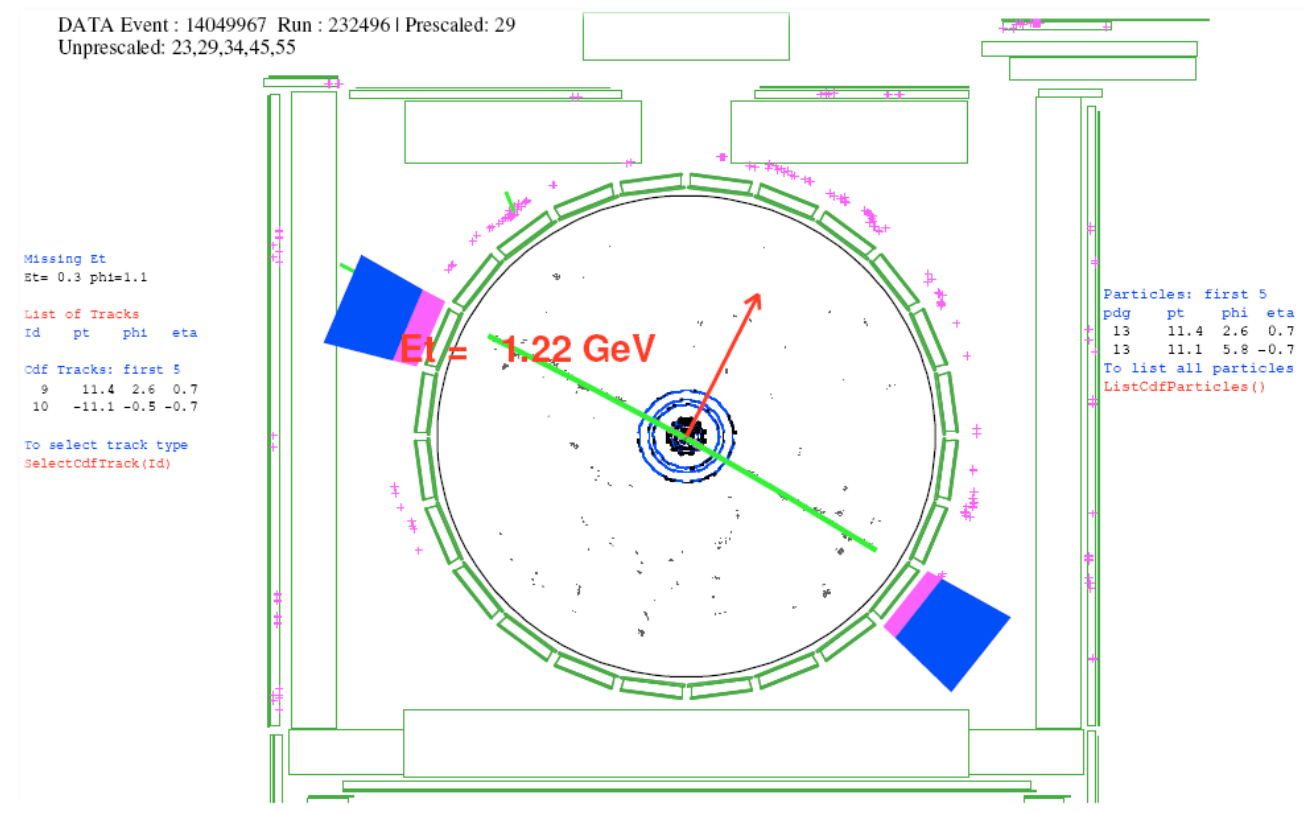

Figure 8.2: A cosmic ray event recorded by the CDF detector. The green line shows the trajectory of the cosmic ray muon and the red arrow indicates the direction of the vector sum of missing transverse energy $\mathbb{E}_{T}$ in the calorimeter. Particle ID 13 represents muon.

The alignment, however, does not correct all wire shifts from their nominal values. We use the difference in $E / p$ for $e^{+}$and $e^{-}$from $W$ decays $\left(\Delta(E / p)_{ \pm}\right)$to further correct high-momentum tracks since $\Delta(E / p)_{ \pm}$should be zero when there are no misalignments. The correction in curvature is parameterized in terms of $\cot \theta$ and $\phi$ as:

$$
\begin{aligned}
\delta c= & a_{0}+a_{1} \cot \theta+a_{2} \cot ^{2} \theta+b_{1} \sin \left(\phi+\phi_{0}\right)+b_{2} \sin (3 \phi+0.5) \\
& +c_{1} \sin (\phi-1.5) \cot \theta+c_{2} \sin (\phi-0.9) \cot ^{2} \theta
\end{aligned}
$$

where the parameter values of $a_{i}, b_{i}$ and $\phi_{0}$ are summarized in Table 8.1. The central values and statistical uncertainties of $a_{i}$ and $b_{i}$ are obtained by fitting the 


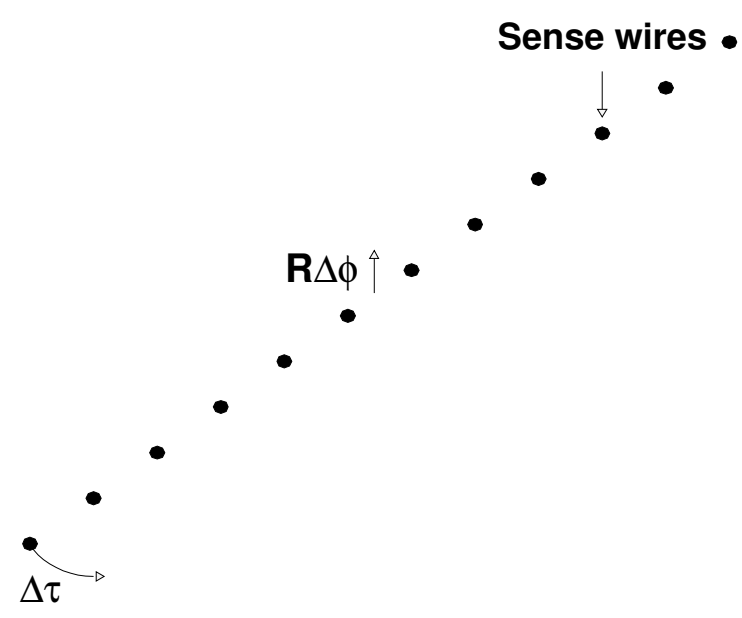

FiguRE 8.3: Illustration of global azimuthal shift $(R \Delta \phi)$ and local tilt around cell center $(\Delta \tau)$ in the COT alignment [8].
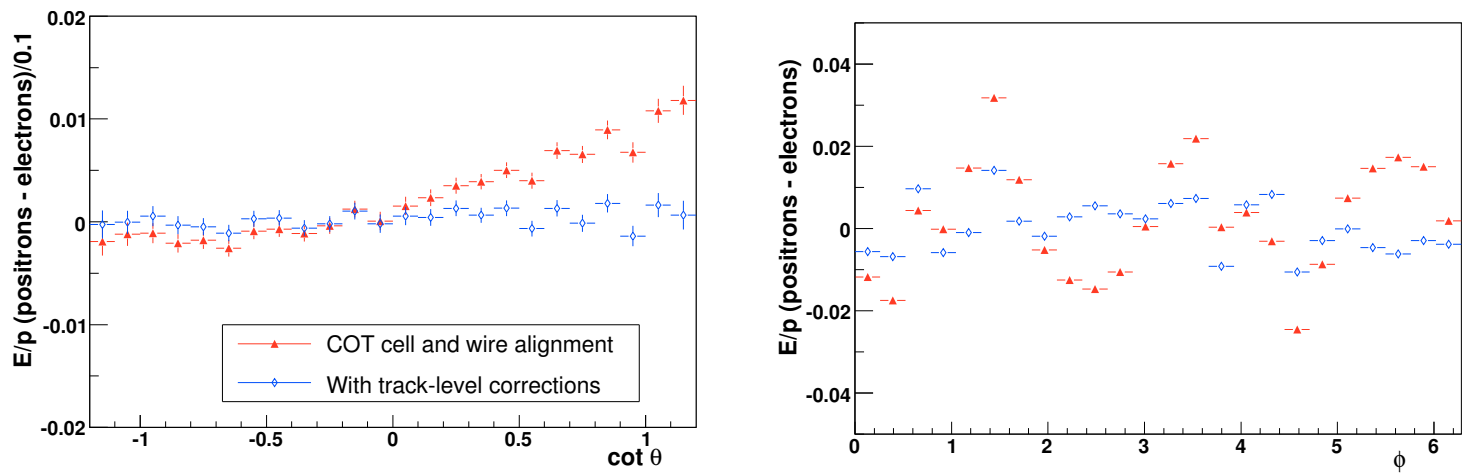

Figure 8.4: The $\cot \theta$ dependence (left) and the $\phi$ dependence (right) of $\Delta(E / p)$. The red triangles are before track correction and the blue circles are after track correction.

$E / p$ difference between $e^{+}$and $e^{-}$from $W$ decays. Figure 8.4 shows the comparison of $\Delta E / p$ before and after applying Eqn. (8.1).

Each term in Eqn. (8.1) corresponds to a physical effect. For example, the first term $a_{0}$ comes from a relative rotation of an end plate's outer edge with respect to its inner edge; the second term $a_{1} \cot \theta$ comes from a relative rotation between the two endplates; the term $b_{1} \sin \left(\phi+\phi_{0}\right)$ arises from a mismeasurement of the beam 
Table 8.1: A list of parameters used to further correct track curvature after alignment.

\begin{tabular}{ccc}
\hline \hline Parameter & Value $\left(\times 10^{-7} \mathrm{~cm}^{-1}\right)$ & Run Range \\
\hline$a_{0}$ & $0.25 \pm 0.04$ & all \\
$a_{1}$ & $1.93 \pm 0.08$ & all \\
$a_{2}$ & $1.21 \pm 0.08$ & all \\
$b_{1}$ & $1.48 \pm 0.21$ & $\leq 212133$ \\
& $-0.91 \pm 0.63$ & $>212133$ \\
$b_{2}$ & $5.92 \pm 0.21$ & all \\
$c_{1}$ & -2.96 & all \\
$c_{2}$ & 3.39 & all \\
\hline$\phi_{0}$ & -1.30 & $\leq 212133$ \\
& 0.20 & $>212133$ \\
\hline \hline
\end{tabular}

position. Out of all the parameters, the uncertainty on $a_{1}$ dominates the impact on $m_{W}[8]$. We vary the $a_{1}$ by its statistical uncertainty and find momentum scale uncertainty of $0.01 \times 10^{-3}$.

Any remaining biases in track parameters are further studied by using $J / \psi \rightarrow \mu \mu$ samples. When those remaining data biases are understood and removed, we can use high statistics $J / \psi$ and $\Upsilon$ samples to calibrate the COT momentum scale.

\subsection{COT Momentum Scale from $J / \psi$ Analysis}

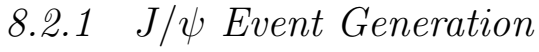

We use Pythia [76] Version 6.157 to generate $J / \psi \rightarrow \mu \mu$ events, where SM-allowed $J / \psi$ production processes are turned on. For each generated $J / \psi \rightarrow \mu \mu$ event, the five track parameters of both muon tracks are written into a vector file, which will be used as an input for the fast detector simulation. To reach the $25 \mathrm{MeV}$ precision goal in $m_{W}$ measurement, we use double precision on the track parameters and, correspondingly, all variables in the fast simulation are using double precision. For the $J / \psi$ analysis, a vector file containing $61.25 \mathrm{M}$ events is generated. Using 
the fact that the width of di-muon invariant mass when convoluted with detector effects is more than 1000 times wider [48] compared with that without any detector effects, we recycle PYTHIA-generated $J / \psi \rightarrow \mu \mu$ events 10 times to mimic the process of generating $612.5 \mathrm{M}$ events. The systematic uncertainty on $m_{W}$ caused by this recycling technique is found to be $0.8 \mathrm{MeV}$ [48], which is negligible compared with the precision we want to reach.

Since the PYTHIA event generator does not simulate QED radiation for quarkonium resonances, we scale each muon's momentum in the fast simulation according to Sudakov form factor, which descibes the leading-log probability distribution for soft photon radiation [77]:

$$
f_{\mu}^{\mu}(x)=\beta(1-x)^{\beta-1}
$$

where

$$
\beta=\frac{\alpha_{e m}}{\pi}\left[\ln \left(Q^{2} / m_{\mu}^{2}\right)-1\right]
$$

with $Q^{2}=m_{J / \psi}^{2}$ for $J / \psi$ analysis and $Q^{2}=m_{\Upsilon}^{2}$ for $\Upsilon$ analysis. $x$ is the energy fraction retained by the muon after soft photon radiation, $f_{\mu}^{\mu}(x)$ is the $\mu \rightarrow \mu$ fragmentation probability at given $x$.

The $p_{T}^{\mu \mu}$ spectrum from PYTHIA sample is significantly softer than that from data as PYTHIA does not model $p_{T}^{\mu \mu}$ distribution well (see the left plot in Figure 8.5). Another reason is our PYTHIA sample only includes prompt $J / \psi$ production while in our data sample about $20 \%$ of the $J / \psi$ mesons are produced from B hadron decays. For the COT momentum scale calibration, the source of $J / \psi$ mesons is not important, but the $p_{T}$ affects the mass resolution and must be tuned. To make the data and the simulation $p_{T}^{\mu \mu}$ spectra agree, the $J / \psi p_{T}$ is increased in a way such that the average $p_{T}$ of the mesons are broadened. The success of tuning is demonstrated in the right plot in Figure 8.5. For all data - Monte Carlo comparisons, the data are represented by blue points while the Monte Carlo is shown as a red histogram. The 
data are sideband-subtracted using $3.01 \mathrm{GeV} / \mathrm{c}^{2}<m_{\mu \mu}<3.15 \mathrm{GeV} / \mathrm{c}^{2}$ as the signal region and $3.17 \mathrm{GeV} / \mathrm{c}^{2}<m_{\mu \mu}<3.31 \mathrm{GeV} / \mathrm{c}^{2}$ as the sideband region.
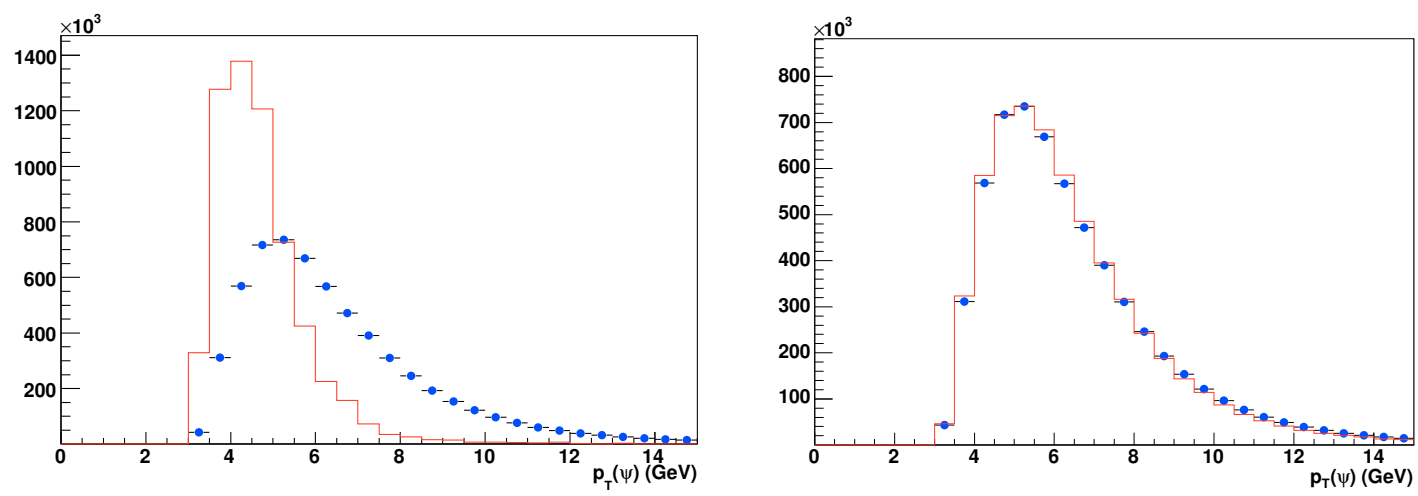

FiguRE 8.5: The $p_{T}^{J / \psi}\left(p_{T}^{\mu \mu}\right)$ distributions before (left) and after (right) tuning.

As shown in the left plot of Figure 8.6, a significant descrepancy between data and $\mathrm{MC}$ is observed in the distribution of the sum of the curvatures $\left(q / p_{T}\right)$ of the two muons. To make data and MC agree, we scale the cotangent of the muon decay angle $\cot \theta^{*}$ by a factor of 1.3 , where $\theta^{*}$ is the muon angle in the rest frame of $J / \psi$ (see Figure 8.7). This scaling changes the fraction of decays in the $J / \psi$ momentum direction and increases the asymmetry between the $p_{T}$ of the two decay muons in each event. See the right plot in Figure 8.6 for data-MC comparison after tuning. Figures $8.8 \sim 8.13$ show additional kinematic distribution comparisons of interest between the data and MC.

\subsection{2 $\mathrm{J} / \psi$ Analysis}

As illustrated in Figure 8.1, the calibration of the momentum scale using about 7.5 million $J / \psi \rightarrow \mu \mu$ events is done in three steps.

Firstly, the data bias due to non-uniform magnetic field is corrected. A nonuniformity of the magnetic field can be expected at high values of $|\cot \theta|$ of the muon tracks since it is reasonable that the magnetic field at the edge of the COT along the $z$ direction is no longer uniform and the extent of uniformity varies depending 

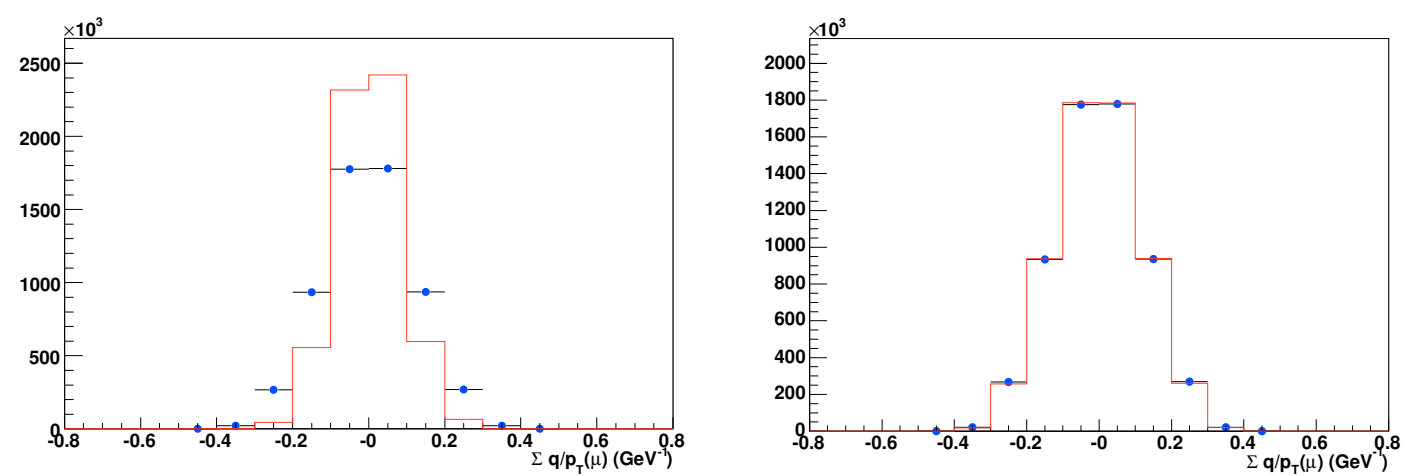

Figure 8.6: The distributions of the sum of $\left(q / p_{T}\right)$ over two decay muons before (left) and after (right) tuning.

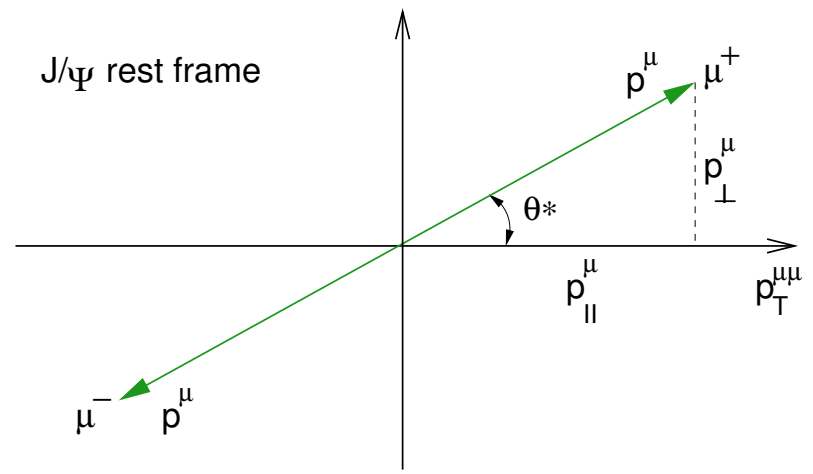

FiguRE 8.7: Kinematics in the quarkonium rest frame.
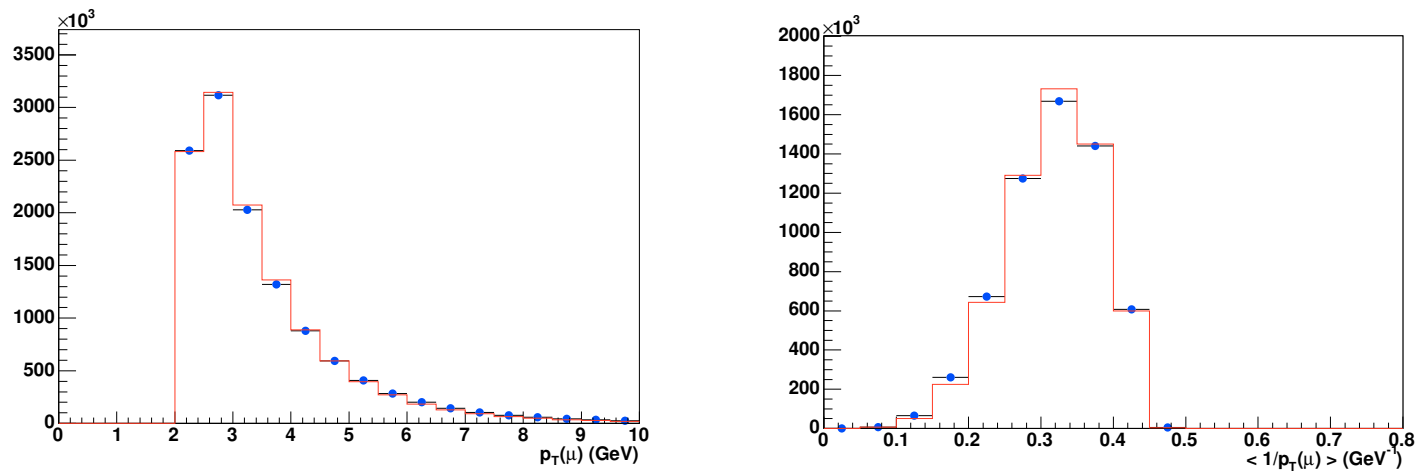

Figure 8.8: Data and simulation distributions of the muon transverse momentum $p_{T}^{\mu}$.

Figure 8.9: Data and simulation distributions of the average unsigned curvature of the two muons.

on the region in COT. We study this non-uniformity effect by selecting $J / \psi$ events with the two decaying muons close to each other in the polar angle $(|\Delta \cot \theta|<0.1)$ 


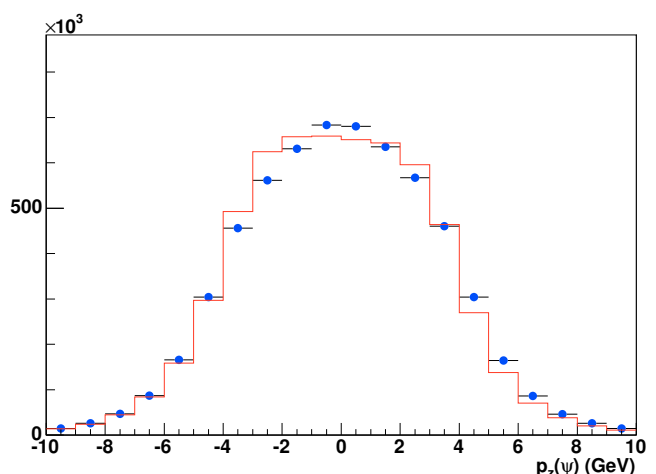

Figure 8.10: Data and simulation distributions of the $J / \psi$ momentum in $z$ direction.

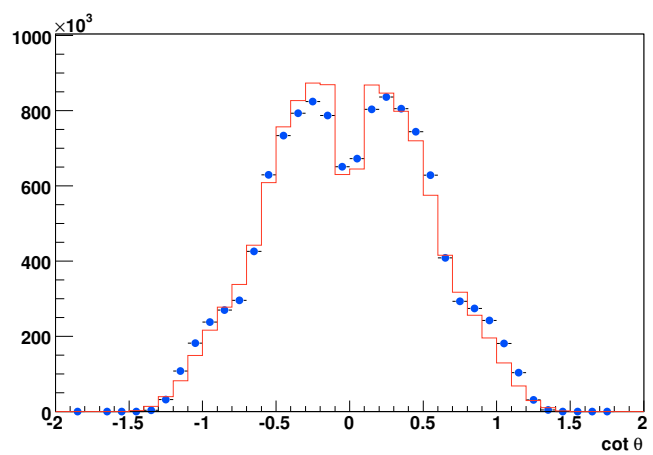

Figure 8.12: Data and simulation distributionss of $\cot \theta$ of the decay muons.

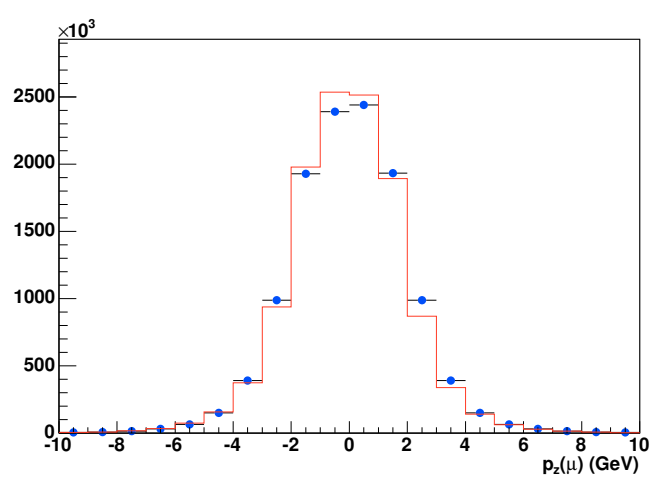

FiguRE 8.11: Data and simulation distributions of $p_{z}$ of the decay muons.

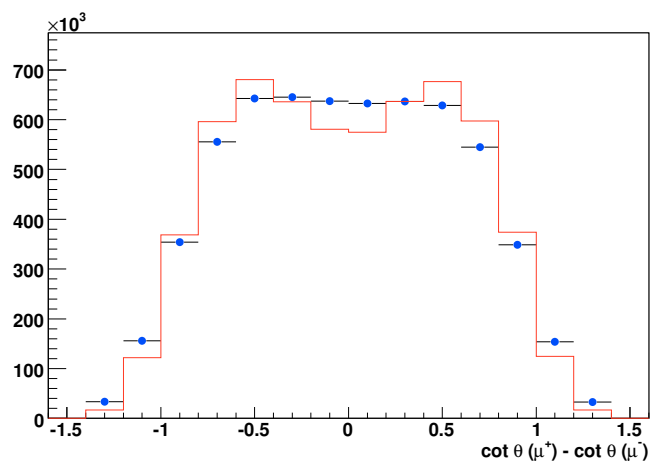

Figure 8.13: Data and simulation distributions of $\Delta \cot \theta$ of the decay muons.

where $\Delta \cot \theta=\cot \theta_{\mu^{+}}-\cot \theta_{\mu^{-}}$, see Figure 8.14 for illustration of such events.
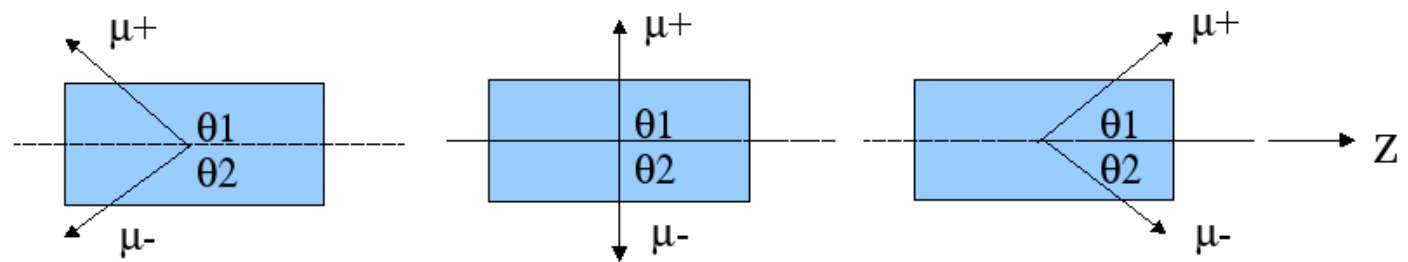

FigURE 8.14: Illustration of events used to study non-uniform magnetic field within the COT.

The fitted $\Delta p / p$ is then studied as a function of $\langle\cot \theta\rangle=\frac{\cot \theta_{\mu^{+}}+\cot \theta_{\mu^{-}}}{2}$. If the 
magnetic field is uniform within the COT, then the fitted $\Delta p / p$ should be independent of $\langle\cot \theta\rangle$. The left plot of Figure 8.15 shows the variation of fitted $\Delta p / p$ with $\langle\cot \theta\rangle$, fitted to a parabola, where a clear dependence is observed. By making the following correction in the data

$$
p_{T} \rightarrow\left(1-0.00019 \cdot \cot \theta+0.00034 \cdot \cot ^{2} \theta\right) \cdot p_{T}
$$

eliminates both the quadratic and the linear dependences of $\Delta p / p$ on $\langle\cot \theta\rangle$. This is demonstraed in the right plot of Figure 8.15. This magnetic field correction is applied as a default correction to all the $J / \psi, \Upsilon, W$ and $Z$ samples since this effect affects all charged leptonic decays.
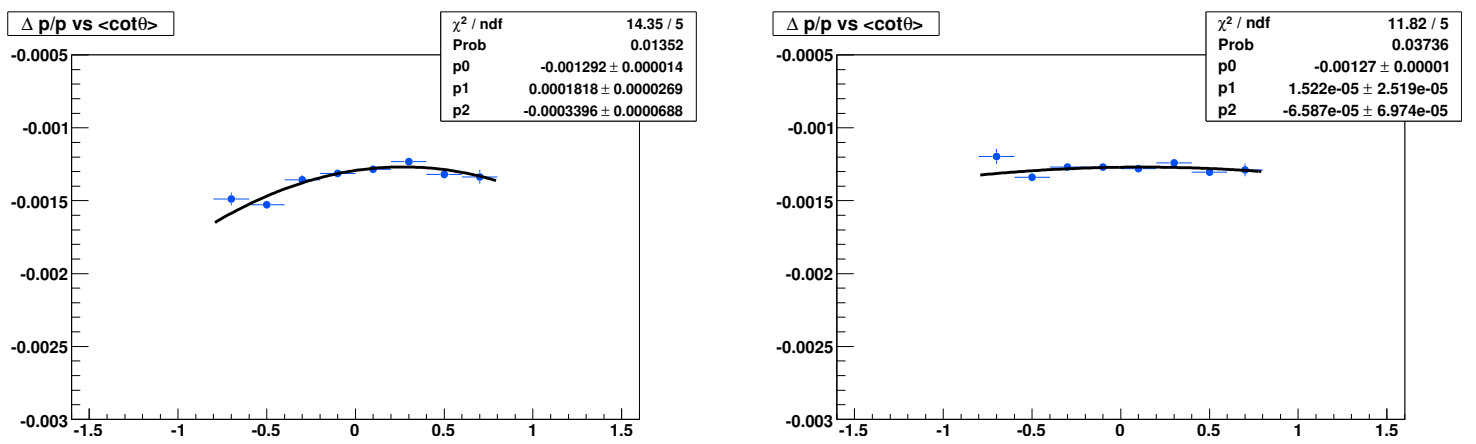

Figure 8.15: Fitted $\Delta p / p$ as a function of $\cot \theta$ with $|\Delta \cot \theta|<0.1$, before corrections (left) and after corrections (right), fitted to a parabola.

As a second step, the data bias due to the COT misalignment is corrected. The variable which can be used to explore this data bias is the difference in $\cot \theta$ between the positively and negatively charged muon tracks. If there is no bias in the data, the fitted $\Delta p / p$ should be independent of $\Delta \cot \theta$. Figure 8.16 shows the decay topology of events which can be used to study the misalignment effects.

The left plot in Figure 8.17 shows the variation of the fitted $\Delta p / p$ with $\Delta \cot \theta$. Since $\Delta p / p$ in our templates varies in the range $(-0.0025,0)$, results outside of this range will take the boundary value -0.0025 or 0 . A quadratic dependence of $\Delta p / p$ 

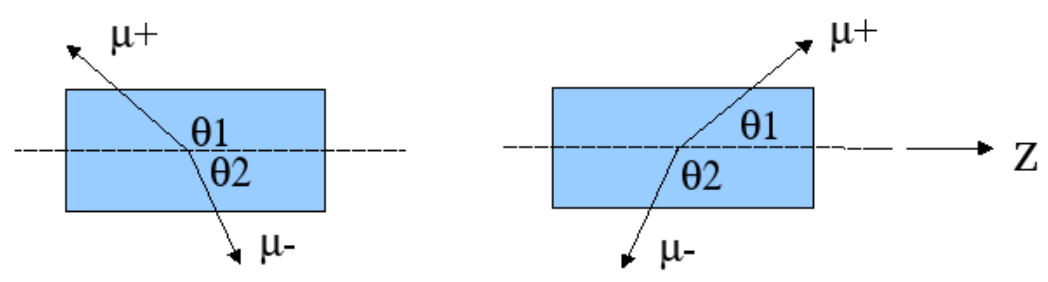

FiguRE 8.16: Illustration of events used to study COT misalignment effects.

in $\Delta \cot \theta$ is induced by a scale factor on $\cot \theta$, which can be caused by a small deviation of the stereo angles from their nominal values. A linear dependence of $\Delta p / p$ in $\Delta \cot \theta$ can be caused by a relative rotation of the east and the west COT endplates. After iterating, we find scaling the $\cot \theta$ by a factor of 1.001640 ( $z$-scale) removes the quadratic variation, and reducing the curvature $c$ by $1.320 \times 10^{-7} \cdot \cot \theta$ $(\delta c)$ removes the linear variation:

$$
\begin{gathered}
\cot \theta \rightarrow(1.001640 \pm 0.000018) \cdot \cot \theta \\
c \rightarrow c-(1.320 \pm 0.092) \cdot 10^{-7} \cot \theta
\end{gathered}
$$

The right plot in Figure 8.17 shows the fitted $\Delta p / p$ as a function of $\cot \theta$ before (left) and after (right) the above corrections. The error terms correspond to $1 \sigma$ statistical error of the $z$-scale and $\delta c$.

With the data biases removed, we then study the material description in the detector simulation. If the material is correctly simulated, then the fitted $\Delta p / p$ should be independent of the average of the inverse transverse momentum $\left(\left\langle 1 / p_{T}^{\mu}\right\rangle\right)$ from the two decay muons. It can be shown that the relationship between $\Delta p / p$ and $\left\langle 1 / p_{T}^{\mu}\right\rangle$ is approximately linear and the slope term of this linear relationship is approximately the ionization energy loss (see Appendix C). We find that scaling the amount of ionizing material in the simulation by a factor of $1.043\left( \pm 0.008_{\text {stat }}\right)$ eliminates the dependence of $\Delta p / p$ on $\left\langle 1 / p_{T}^{\mu}\right\rangle$. Figure 8.18 shows the variation of 

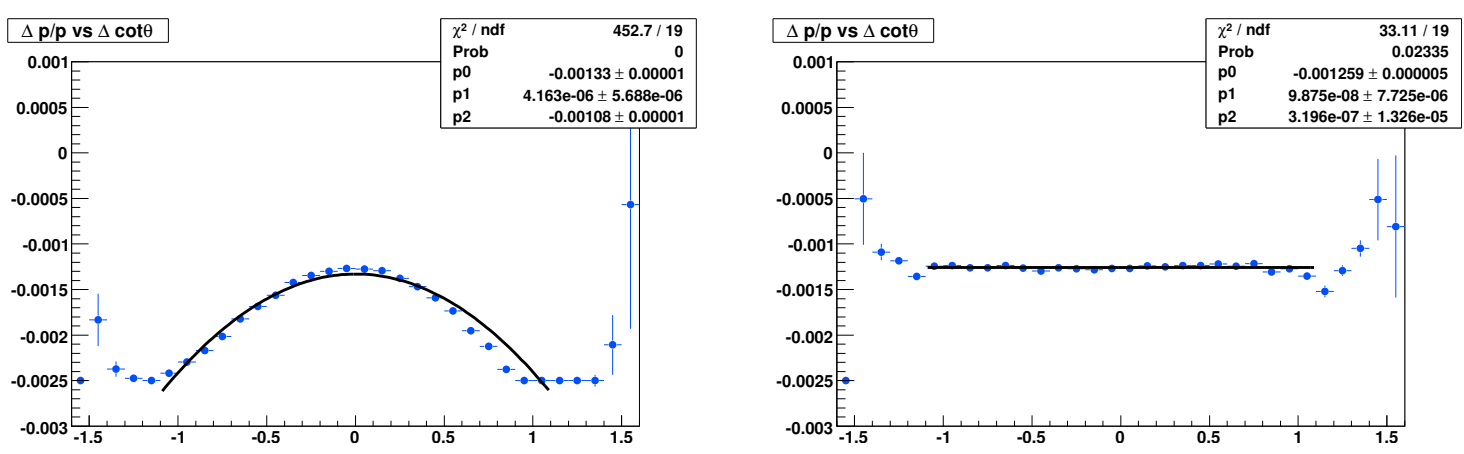

Figure 8.17: Fitted $\Delta p / p$ as a function of $\Delta \cot \theta$, before corrections (left) and after corrections (right), fitted to a parabola.

$\Delta p / p$ as a function of $\left\langle 1 / p_{T}^{\mu}\right\rangle$ with the material scale factor 1.043 applied. Since

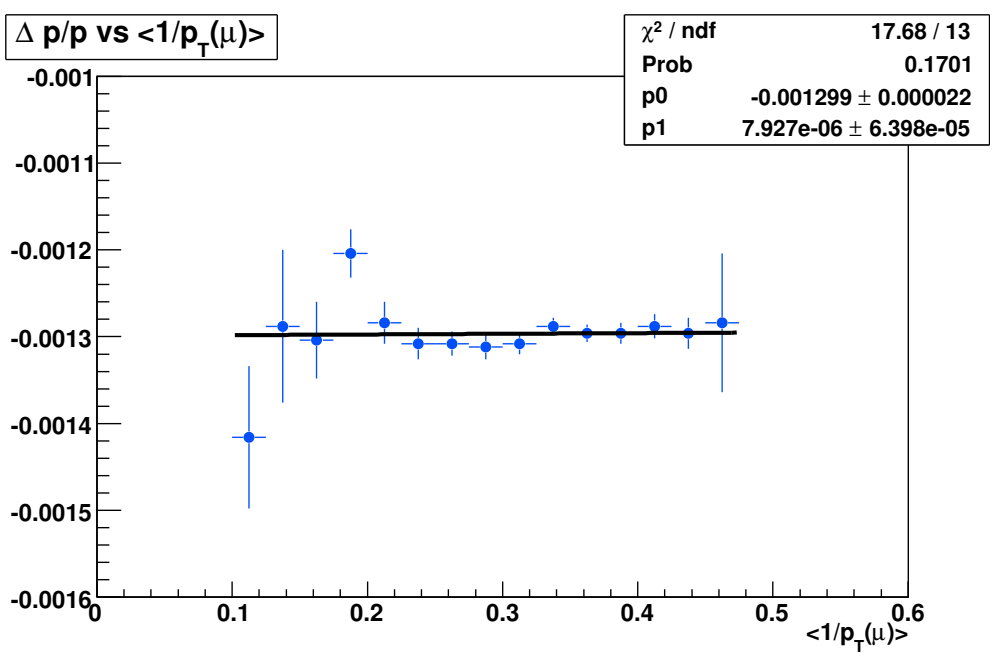

Figure 8.18: Fitted $\Delta p / p$ as a function of $\left\langle 1 / p_{T}^{\mu}\right\rangle$, fitted to a straight line.

the average $p_{T}$ for $W$ and $Z$ decay muons is about $40 \mathrm{GeV} / \mathrm{c}$, which corresponds to $\left\langle 1 / p_{T}^{\mu}\right\rangle \sim 0.025$, we extrapolate the fitting line in Figure 8.18 to the high $p_{T}^{\mu}$ where $\left\langle 1 / p_{T}^{\mu}\right\rangle \sim 0$ to estimate the momentum scale for the $W$ muons. This procedure results in a high accuracy in the track momentum calibration. The extraplated value is found to be $\Delta p / p=\left(-1.299 \pm 0.004_{\text {stat }} \pm 0.022_{\text {slope } / \text { material }}\right) \cdot 10^{-3}$. The first uncertainty is the statistical error in the absence of any uncertainty from the extrapolation to zero 
curvature. We obtain this by setting the slope term of the linear fitting function to $7.927 \cdot 10^{-6}$ then performing a constant fit. We quote the error on the constant term $0.004 \cdot 10^{-3}$ as the statistical error. The second uncertainty is due to the statistical error on the slope, which propagates as an additional uncertainty on the intercept. We interpret the slope error as the ionization energy loss uncertainty. Fit in each $\left\langle 1 / p_{T}^{\mu}\right\rangle$ bin which contributes to Figure 8.18 is shown in Appendix D. There we can see the mass resolution improves as the averaged momentum decreases (or $\left\langle 1 / p_{T}^{\mu}\right\rangle$ increases). The fit window is thus adjusted to cover the di-muon invariant mass peak region.

\subsubsection{Systematic Uncertainties in $J / \psi$ Analysis}

The dominant systematic uncertainty on the $J / \psi$ momentum scale analysis is from QED radiation off the muons from $J / \psi$ decays. As shown earlier, the variation of factorization term Q in Eqn. (8.3) changes the energy retained by the muon after QED radiation. It thus changes the shape of the low-tail region of $J / \psi$ mass fits. To estimate this QED/energy loss uncertainty, we vary $Q$ to minimize the summed $\chi^{2}$ from all $J / \psi$ mass fits in all the $15\left\langle 1 / p_{T}^{\mu}\right\rangle$ bins (see Appendix D). We find applying $Q=1.46 m_{J / \psi}$ minimizes the $\sum \chi^{2}$ and reduces $\sum \chi^{2} /$ d.o.f from $2930 / 1234$ to $2577 / 1234$. To eliminate the correlation between the material scale uncertainty and the radiative correction uncertainty, we retune the material scale for the case of $Q=1.46 m_{J / \psi}$. We find when $Q=1.46 m_{J / \psi}$, the momentum scale gets shifted by $\left.0.080\left(\cdot 10^{-3}\right)\right)$ as compared to the nominal value of $Q=m_{J / \psi}$. We quote this shift in $\Delta p / p$ as the systematic uncertainty associated with QED and energy loss model. For ease of discussion, we will use parts-per-thousands (PPT) as a substitute for $10^{-3}$.

The nonuniformity of the magnetic field comes as the second largest source of systematic uncertaities. We study this uncertainty by applying the same magnetic field (B-field) correction obtained from $J / \psi$ analysis to $W$ data, and evaluating the 
shift in $m_{W}$. We then compare the $m_{W}$ shift from $W$ data with the $\Delta p / p$ shift in the $J / \psi$ data. Both shifts are in the same direction and can cancel partly. The residual shift in the $m_{W}$, taken as the difference between the two mass shifts, corresponds to $\Delta p / p^{\text {shift }}=0.064$ PPT. Since this amount of change in momentum scale covers $100 \%$ net effect of the B-field correction, we quote half of this change as the associated systematic uncertainty.

The systematic uncertainty arising from the material description of ionization energy loss is taken as the uncertainty of the constant term $p_{0}$ in Figure 8.18. We quantify the uncertainty due to COT hit resolution by varying the resolution scale factor $h_{0}$, which is applied to SL-dependent resolution model, by $\pm 1.7 \%$ from its best value 1.052. The COT hit resolution scale factor $h_{0}=1.052$ is determined by minimizing the sum of $\chi^{2}$ of the five highest-momentum bins in $\left\langle 1 / p_{T}^{\mu}\right\rangle$, which are the most sensitive to hit resolution. The uncertainty $1.7 \%$ is obtained from $6 \% / \sqrt{12}$, where the $6 \%$ is the maximum variation of $\operatorname{per}-\left\langle 1 / p_{T}^{\mu}\right\rangle$-bin $h_{0}$, and the $\sqrt{12}$ comes from the definition of standard deviation of a uniform distribution $x \sim U(a, b)$ : $\sigma_{x}=(b-a) / \sqrt{12}$. Brief descriptions of all other systematic sources, together with their corresponding values, are presented in Table 8.2.

Since all the above systematic uncertainties are physically independent, according to the law of error propagation [32], we can add them in quadrature to obtain the $\Delta p / p$ from $J / \psi$ analysis to be $\Delta p / p=\left(-1.299 \pm 0.004_{\text {stat }} \pm 0.092_{\text {syst }}\right) \cdot 10^{-3}$. Since $m_{J / \psi}=3096.880 \mathrm{MeV}$ is used in the PYTHIA event generation while the current world averaged $J / \psi$ mass is $m_{J / \psi}=3096.916 \mathrm{MeV}$, we correct the above $\Delta p / p$ central value by

$$
\delta(\Delta p / p)=\frac{3096.880-3096.916}{3096.916}=-0.0116 \cdot 10^{-3}
$$


Table 8.2: Statistical and systematic uncertainties on $\Delta p / p$ from the $J / \psi$ analysis.

\begin{tabular}{c|c|l}
\hline \hline Source & $J / \psi\left(\cdot 10^{-3}\right)$ & \multicolumn{1}{c}{ Method } \\
\hline Statistical & 0.004 & Statistical uncertainty of constant $\Delta p / p$ vs $1 / p_{T}$ \\
\hline QED/energy loss & 0.080 & Vary $Q$ in Sudakov form factor, minimize $\chi^{2}$ \\
Magnetic field & 0.032 & Change in $m_{W}$ not covered by B-field correction \\
Material & 0.022 & Statistical uncertainty of slope of $\Delta p / p$ vs $1 / p_{T}$ \\
Resolution & 0.020 & Vary hit resolution scale factor $h_{0}$ by $\sim 1.7 \%$ \\
Backgrounds & 0.011 & Vary linear background parameters \\
Misalignment & 0.009 & Vary $z$-scale, $\delta c$ corrections \\
Trigger efficiency & 0.004 & Vary muon $p_{T}$ cuts by $200 \mathrm{MeV}$ \\
Fitting window & 0.004 & Vary fit windows $20 \%$ larger $/$ smaller \\
$\Delta p / p$ step size & 0.002 & Quote $\Delta p / p$ step size in MC templates \\
World average & 0.004 & $\Delta m_{J / \psi}^{\mathrm{PDG}} / m_{J / \psi}^{\mathrm{PDG}}$ \\
\hline Total systematic & 0.092 &
\end{tabular}

Thus the final momentum scale from $J / \psi$ analysis is:

$$
\left(\frac{\Delta p}{p}\right)_{J / \psi}=\left(-1.311 \pm 0.004_{\mathrm{stat}} \pm 0.092_{\mathrm{syst}}\right) \cdot 10^{-3}
$$

\subsection{COT Momentum Scale from $\Upsilon$ Analysis}

As mentioned before, the $\Upsilon(1 S)$ resonance acts as an intermediate reference point to connect the momentum scale from low momentum $J / \psi$-decay muons with that from high momentum $W$-decay muons. With the muon $p_{T}^{\mu}$ from $\Upsilon \rightarrow \mu \mu$ decays being higher than that from $J / \psi \rightarrow \mu \mu$ decays, the momentum scale from $\Upsilon$ analysis is relatively less sensitive to the description of QED radiation and ionizing material, thus leading to comparable precision on the momentum scale from the $J / \psi$ analysis. Figure 8.19 shows the dimuon invariant mass spectrum between $8.8 \mathrm{GeV} / \mathrm{c}^{2}$ and 11.2 $\mathrm{GeV} / \mathrm{c}^{2}$ with a fit to three Gaussian distributions on top of a linearly parameterized background. The three bumps from low to high invariant mass values are $\Upsilon(1 S)$, 


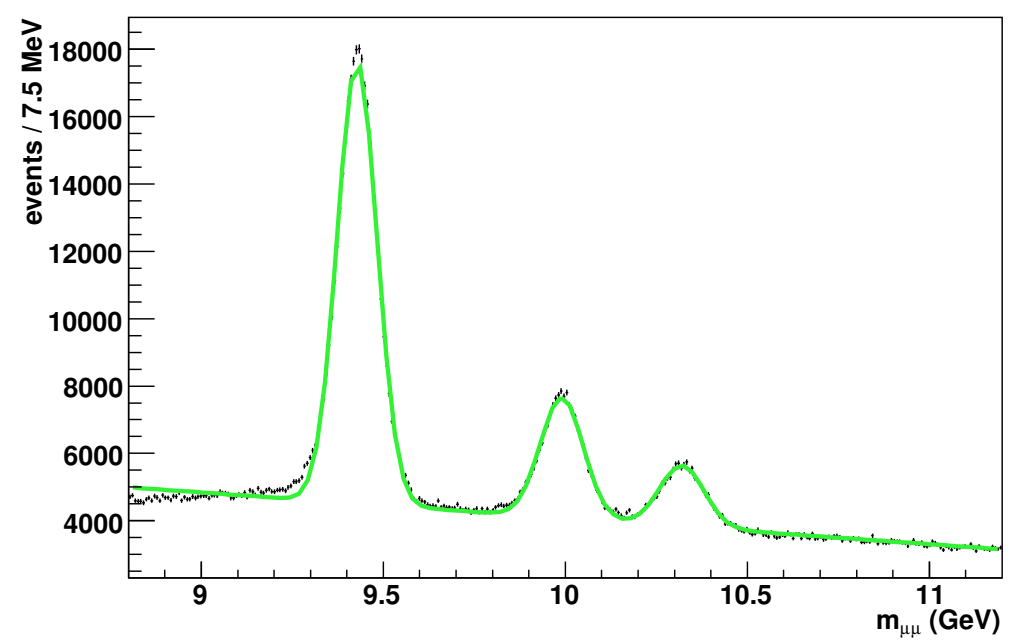

FiguRE 8.19: Dimuon mass spectrum with a fit of three Gaussian distributions and a linear background

$\Upsilon(2 S)$ and $\Upsilon(3 S)$ resonances, respectively. The $\Upsilon(4 S)$ resonance is not shown in Figure 8.19 as it is outside of the plotting window. Since the $\Upsilon(1 S)$ data sample size is much larger than that of $\Upsilon(2 S)$ and it has been shown that the addition of $\Upsilon(2 S)$ would only improve the statistical sensitivity by $10 \%$, we only use $\Upsilon(1 S)$ resonance to estimate the momentum scale. Similar to $J / \psi$ analysis, the $\Upsilon$ invariant mass templates are produced with DukeSim using generator-level vector files from PYTHIA.

For the $\Upsilon$ analysis we apply the same track cuts as for the $W$ and $Z$ selection, requiring at least $3 / 3$ axial/stereo SLs with $\geq 5$ hits and $\geq 25$ hits in all the axial/stereo SLs. We apply the same good run list as is used in the $W$ and $Z$ analysis. The same magnetic field non-uniformity correction derived from $J / \psi$ sample is applied to both the beam-constrained and non-beam-constrained $\Upsilon \rightarrow \mu \mu$ data. We apply the same material scale factor 1.043 from $J / \psi$ analysis to the $\Upsilon$ analysis. In the simulation, simple fiducial cuts are made to emulate the muon detector acceptance. To emulate the different $p_{T}$ cuts applied in the data for CMUP muons vs CMU muons, we imple- 
ment the following scheme in the fast simulation: $60 \%$ of the time the higher $p_{T}$ cut is applied to a randomly chosen muon, and $40 \%$ of the time the muon generated with higher $p_{T}$ has the higher cut applied. The generator-level muon kinematics are tuned to match the data distributions in the same way as implemented for the $J / \psi$ mesons: we increase the rapidity of the $\Upsilon$ along its momentum vector by $\Delta y_{\Upsilon}=k \cdot y_{\Upsilon}$ where $k=0.1$ for half of the mesons and $k=0.6$ for the other half. With this scheme we are able to match the muon $p_{T}$ distribution in the data well, as shown in Fig. 8.22. Unlike the $J / \psi$, there is no need to tune the $\theta^{*}$ distribution for the $\Upsilon$ sample from PYTHIA.

The acceptance model for the CMUP-CMX trigger uses $z$ cuts motivated by the boundaries of the muon subdetectors. The kinematic comparisons of data and Monte Carlo are shown in Figure 8.20 to Figure 8.27, where the data have been background-subtracted. The signal region is chosen to be from 9.30 to $9.56 \mathrm{GeV}$ and the sidebands are taken from the lower sideband region $(9.17$ to $9.3 \mathrm{GeV})$ and the upper sideband region (9.56 to $9.69 \mathrm{GeV})$.

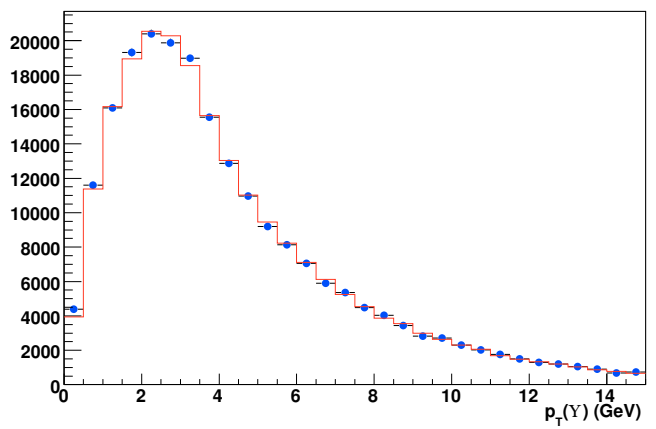

Figure 8.20: Comparison of data and Monte Carlo of the $\Upsilon(1 \mathrm{~S}) p_{T}$ distribution.

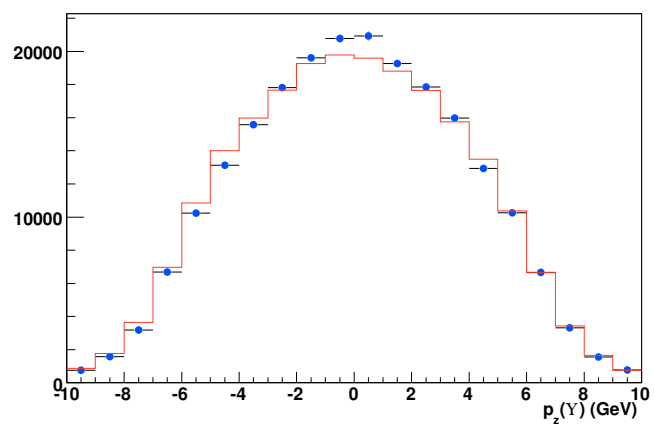

Figure 8.21: Comparison of data and Monte Carlo of the $\Upsilon(1 \mathrm{~S}) p_{z}$ distribution.

The fitting region of the $\Upsilon(1 S)$ invariant mass is taken to be $9.28 \mathrm{GeV} / \mathrm{c}^{2}<m_{\Upsilon}<$ $9.58 \mathrm{GeV} / \mathrm{c}^{2}$ for beam-constrained tracks and $9.245 \mathrm{GeV} / \mathrm{c}^{2}<m_{\Upsilon}<9.615 \mathrm{GeV} / \mathrm{c}^{2}$ for non-beam-constrained tracks, respectively. The latter one is wider because of 


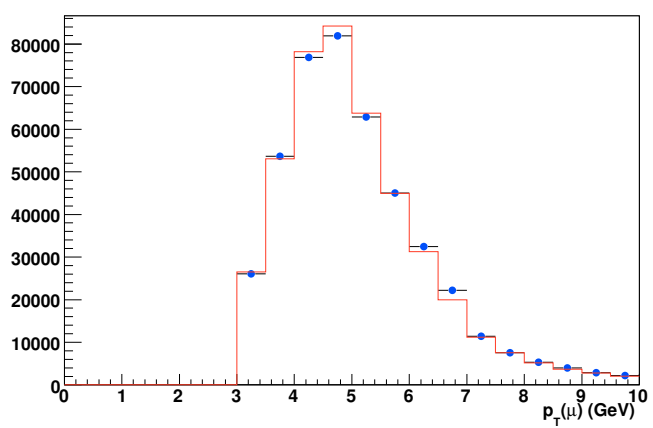

Figure 8.22: Comparison of data and Monte Carlo of the muon candidate $p_{T}$ distribution.

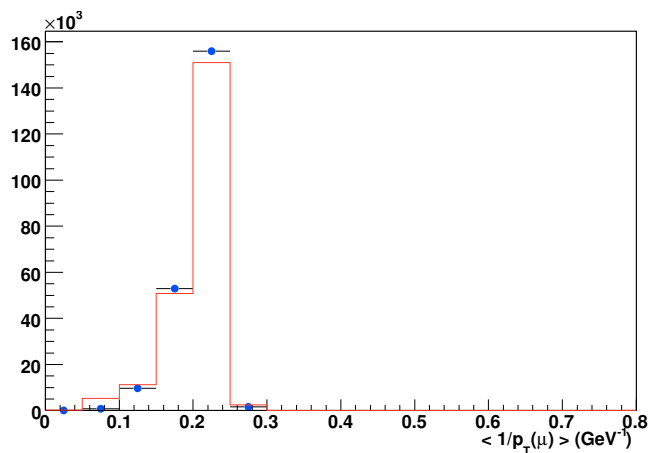

Figure 8.24: Comparison of data and Monte Carlo of the average muon inverse transverse momentum distribution.

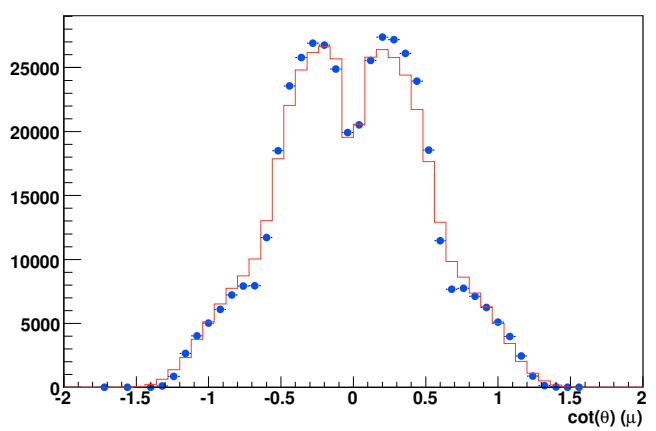

FiguRE 8.26: Data and simulation distributions of $\cot \theta$ of the decay muons.

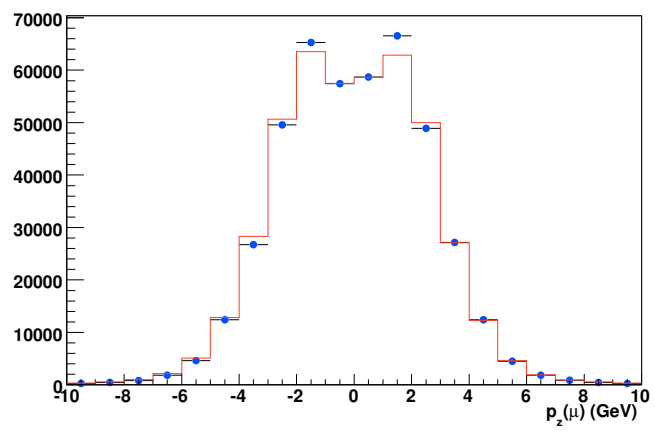

Figure 8.23: Comparison of data and Monte Carlo of the muon candidate $p_{z}$ distribution.

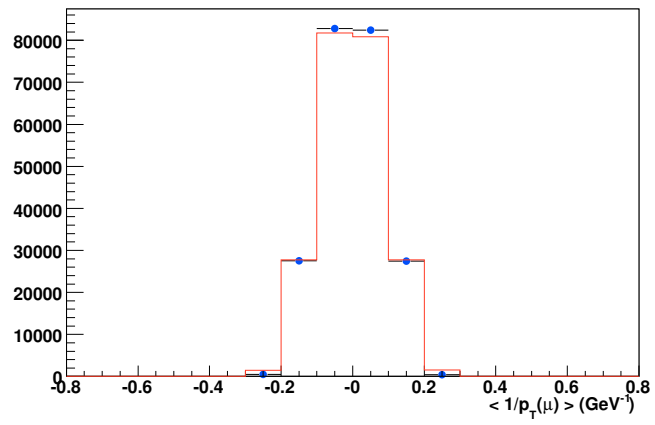

Figure 8.25: Comparison of data and Monte Carlo of the summed muon inverse transverse momentum distribution.

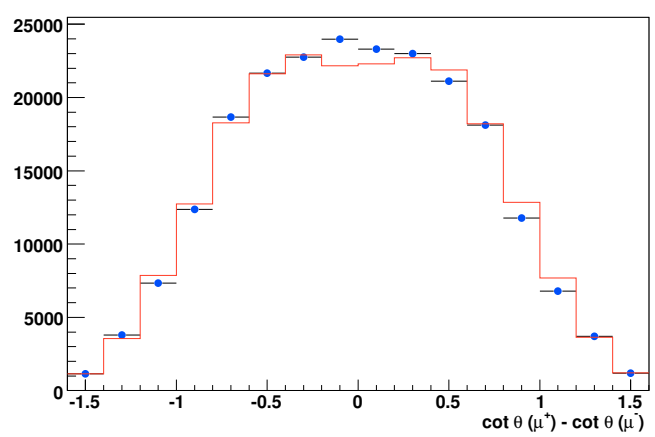

FiguRe 8.27: Data and simulation distributions of $\Delta \cot \theta$ of the decay muons.

the reduced resolution as the beam spot is not used in constraining the tracks. To increase the statistics in the templates used to fit the data, we recycle each generated 
PYTHIA event 10 times since the spread of the invariant mass spectrum mostly comes from the detector resolution.

The COT hit resolution is parameterized by a SL-dependent resolution model. We use non-beam-constrained $\Upsilon \rightarrow \mu \mu$ events to tune the resolution scale factor $h_{0}$, which is applied to the resolution of each SL, to match the width of dimuon mass peak by minimizing the fit $\chi^{2}$. A resolution scale factor $h_{0}=0.978 \pm 0.005_{\text {stat }}$ is found to describe the width of the non-beam-constrained mass distribution. For this value of resolution scale factor, the beamspot size for beam-constrained $\Upsilon$ is found to be $\sigma_{b}=35 \mu \mathrm{m}$ by minimizing the fit $\chi^{2}$.

The COT curvature corrections are derived from the beam-constrained $\Upsilon$ and the non-beam-constrained $\Upsilon$ studies, and are applied to the beam-constrained and non-beam-constrained $\Upsilon$ data respectively. Figure 8.28 shows the fitted $\Delta p / p$ as a function of $\Delta \cot \theta$ for beam-constrained $\Upsilon_{s}$ before any correction is applied. After applying the correction functions to the data:

$$
\begin{aligned}
& \cot \theta \rightarrow(1.00148 \pm 0.00019) \cdot \cot \theta \\
& c \rightarrow c-(2.10 \pm 0.28) \cdot 10^{-7} \cot \theta,
\end{aligned}
$$

the quadratic and the linear dependences are removed, as illustrated in Figure 8.29.

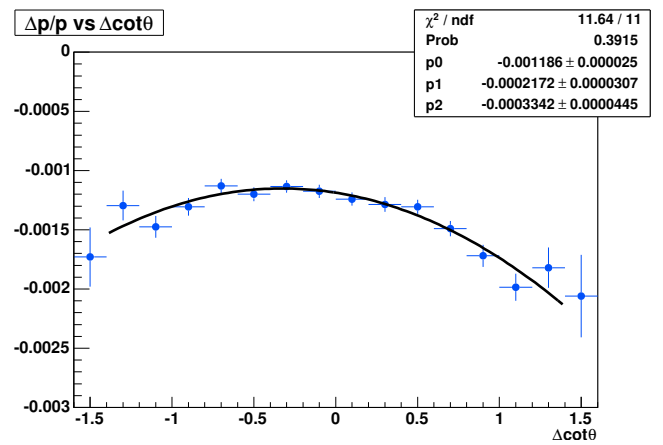

FiguRE 8.28: Fitted $\Delta p / p$ as a function of $\Delta \cot \theta$ for beam-constrained $\Upsilon \mathrm{s}$, before corrections, fitted to a parabola.

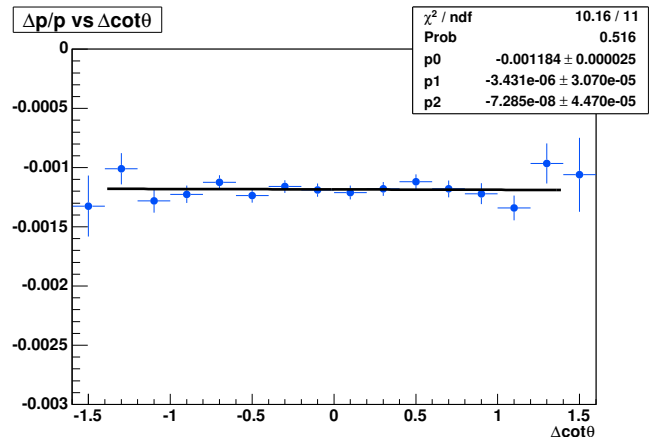

Figure 8.29: Fitted $\Delta p / p$ as a function of $\Delta \cot \theta$ for beam-constrained $\Upsilon_{\mathrm{s}}$, after corrections, fitted to a parabola. 
The same procedure is applied to non-beam-constrained $\Upsilon_{\mathrm{s}}$. Figure 8.30 shows the $\Delta p / p$ dependence on $\Delta \cot \theta$ for non-beam-constrained $\Upsilon \rightarrow \mu \mu$ events before correction. With the following correction function applied in the data

$$
\begin{aligned}
& \cot \theta \rightarrow(1.00160 \pm 0.00025) \cdot \cot \theta \\
& c \rightarrow c-(0.50 \pm 0.36) \cdot 10^{-7} \cot \theta
\end{aligned}
$$

the quadratic and the linear dependences are eliminated, as seen in Figure 8.31.

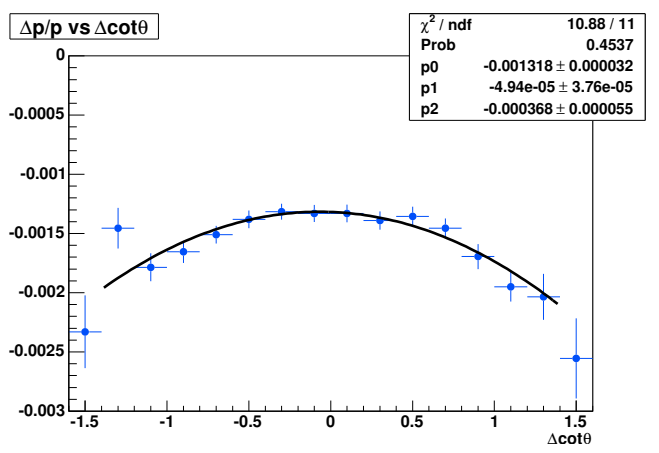

FiguRE 8.30: Fitted $\Delta p / p$ as a function of $\Delta \cot \theta$ for non-beam-constrained $\Upsilon_{\mathrm{s}}$, before corrections, fitted to a parabola.

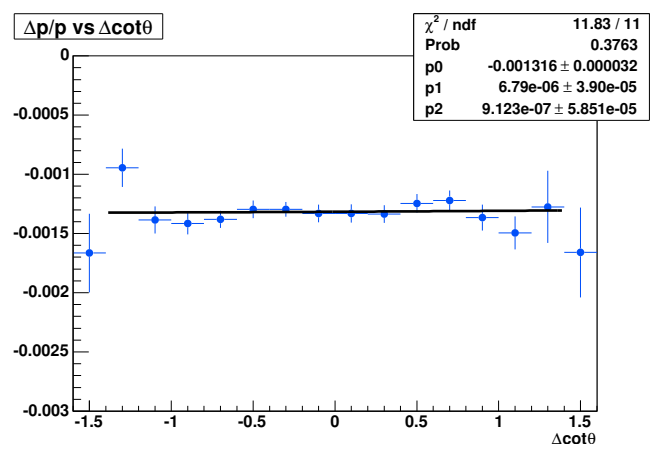

Figure 8.31: Fitted $\Delta p / p$ as a function of $\Delta \cot \theta$ for non-beam-constrained $\Upsilon \mathrm{s}$, after corrections, fitted to a parabola.

Figure 8.32 and 8.33 show the material scale derived from the $J / \psi$ analysis can describe the beam-constrained and non-beam-constrained $\Upsilon$ data well. The entries in 8.32 come from beam-constrained $\Upsilon$ fits in $\left\langle 1 / p_{T}^{\mu}\right\rangle$ bins, which are presented in Appendix E. The corresponding non-beam-constrained $\Upsilon$ fits in $\left\langle 1 / p_{T}^{\mu}\right\rangle$ are summarized in Appendix F.

The fit to the inclusive invariant mass distribution using beam-constrained $\Upsilon_{\mathrm{s}}$ is shown in Figure 8.34, and the fit to the inclusive invariant mass distribution using non-beam-constrained $\Upsilon_{\mathrm{s}}$ is shown in Figure 8.35. The corresponding fitted momentum scale results are $(\Delta p / p)_{B C}=\left(-1.185 \pm 0.020_{\text {stat }}\right) \cdot 10^{-3}$ and $(\Delta p / p)_{N B C}=$ $\left(-1.335 \pm 0.028_{\text {stat }}\right) \cdot 10^{-3}$, respectively. We obtain a combined momentum scale central value from $\Upsilon$ analysis by taking the average of the above two central values 


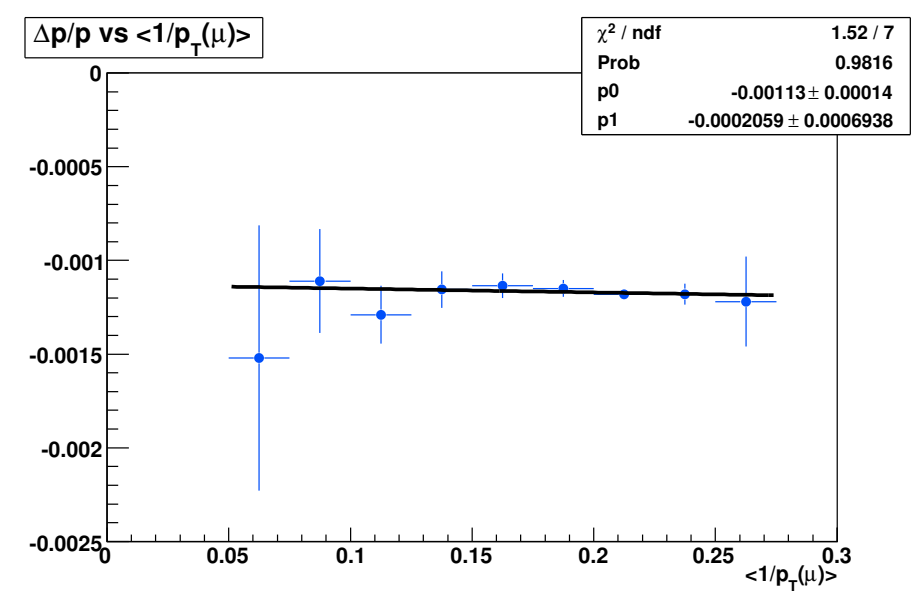

FiguRE 8.32: Fitted $\Delta p / p$ as a function of $\left\langle 1 / p_{T}^{\mu}\right\rangle$ using beam-constrained $\Upsilon \rightarrow \mu \mu$ events, fitted to a straight line. The slope is consistent with zero. The material scale from the $J / \psi$ analysis is included in the $\Upsilon$ simulation. This shows that the $\Upsilon$ data are consistent with the $J / \psi$ data with respect to the ionization energy loss in the detector material.

since all the systematic uncertainties between the beam-constrained and non-beamconstrained cases are completely correlated. We use the statistical uncertainty from the beam-constrained fit $0.20 \times 10^{-3}$ as the statistical uncertainty of the combined $\Upsilon$ result since it is more precise.

The systematic uncertainties on the momentum scale deviation from unity, derived from the $\Upsilon$ analysis, are shown in Table 8.3. The biggest systematic uncertainties are from QED radiative effects, magnetic field nonuniformities and ionizing material. Since $\Upsilon$ sample does not have the statistical power to show any lineshape mismatch at low mass, to estimate the systematic uncertainty in the $\Upsilon$ analysis due to $\mathrm{QED}$ radiation, we scale the factorization term $Q$ in the Sudakov form factor by the same factor of 1.46 , which is found to give the best-fit to the data in the $J / \psi$ analysis. The systematic uncertainty arising from the non-uniformity of the magnetic field is obtained in the same way as in the $J / \psi$ analysis. We apply to $\Upsilon$ data and $W$ data the same $J / \psi$-derived B-field correction. The shift in $m_{W}$ and the shift in the momentum scale partly cancels since they are in the same direction. Because this 


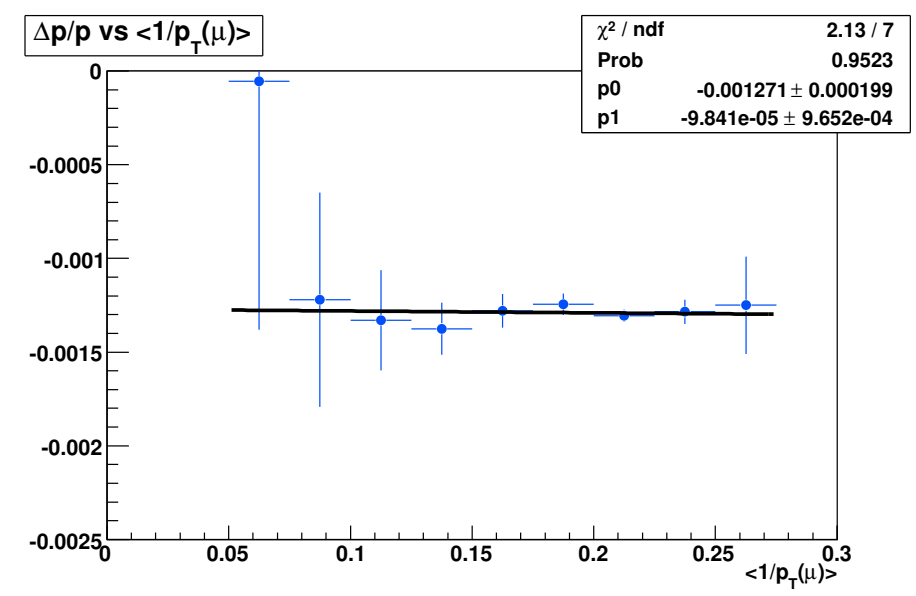

FiguRE 8.33: Fitted $\Delta p / p$ as a function of $\left\langle 1 / p_{T}^{\mu}\right\rangle$ using non-beam-constrained $\Upsilon \rightarrow \mu \mu$ events, fitted to a straight line. The slope is consistent with zero. The material scale from the $J / \psi$ analysis is included in the $\Upsilon$ simulation. This shows that the $\Upsilon$ data are consistent with the $J / \psi$ data with respect to the ionization energy loss in the detector material.

difference covers $100 \%$ effect of B-field non-uniformity, we quote half of its value as the associated systematic uncertainty. To obtain the systematic uncertainty caused by ionizing material, we apply the material scale factor $(1.043 \pm 0.008)$ obtained from $J / \psi$ and vary by its statistical uncertainty. Other systematic uncertainties are obtained in a similar way as those in $J / \psi$ analysis and are also briefly described in the last column of Table 8.3. The only one that is different from $J / \psi$ description is the way to quantify resolution effects. In $\Upsilon$ analysis, we estimate this systematic uncertainty using the beam-spot size $\sigma_{b}$. We find $\sigma_{b}=35 \mu \mathrm{m}$ best matches the lineshape of beam-constrained $\Upsilon$ mass spectrum. This beam-spot size is different from $\sigma_{b}=43 \mu m$, which is derived from the $Z \rightarrow \mu \mu$ sample. We quote the change in $\Delta p / p$ as the systematic uncertainty due to the resolution model.

Combining the systematic uncertainties using the law of error propagation, we obtain the momentum scale from the $\Upsilon$ analysis:

$$
\left(\frac{\Delta p}{p}\right)_{\Upsilon}=\left(-1.260 \pm 0.020_{\text {stat }} \pm 0.101_{\text {syst }}\right) \cdot 10^{-3}=(-1.260 \pm 0.103) \cdot 10^{-3}
$$




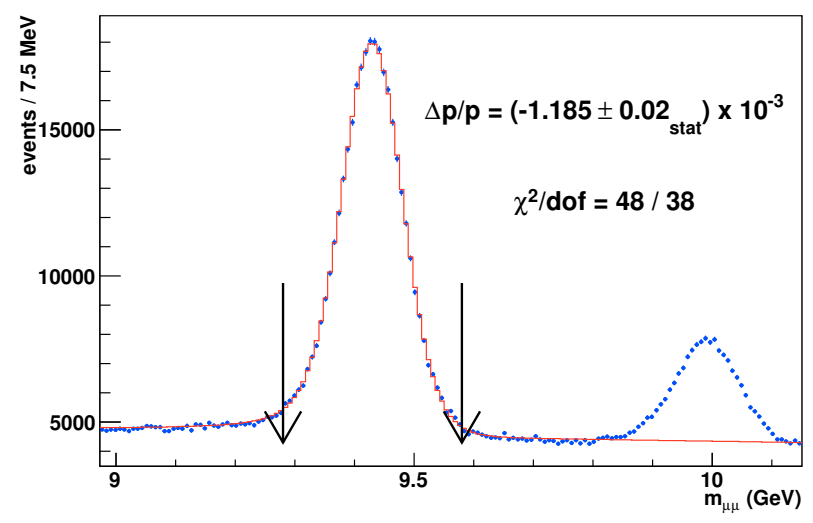

FiguRE 8.34: The invariant mass distribution with the best fit simulated template and the extracted momentum scale using beam-constrained track quantities.

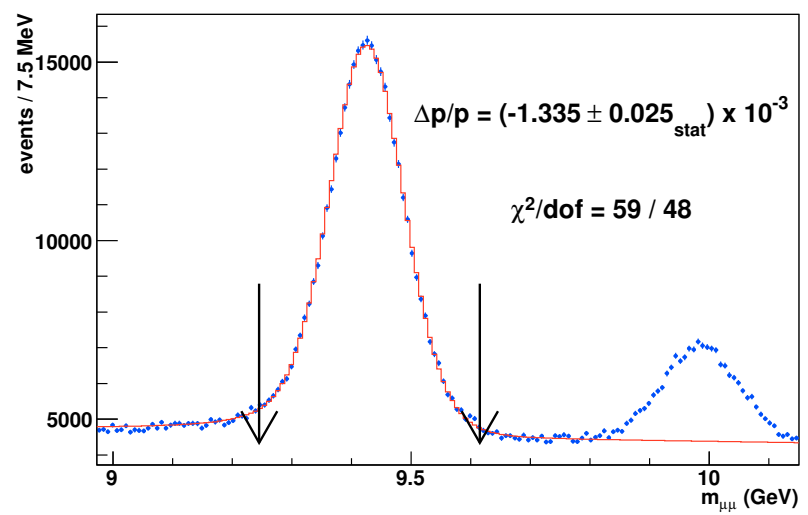

FiguRE 8.35: The invariant mass distribution with the best fit simulated template and the extracted momentum scale using non-beam-constrained track quantities.

As a cross-check we split the $\Upsilon$ sample into two similar-sized sub-samples to check for possible time variation. We fit the beam-constrained dimuon invariant mass distribution because it is statistically more precise. Table 8.4 shows the fit results for $\Delta p / p$ in the two run ranges and in the two instantaneous luminosity bins, respectively. No run dependence or instantaneous luminosity dependence is observed. 
Table 8.3: Statistical and systematic uncertainties on $\Delta p / p$ from the $\Upsilon$ analysis.

\begin{tabular}{|c|c|c|}
\hline Source & $\overline{\Upsilon\left(\cdot 10^{-3}\right)}$ & Method \\
\hline Statistical & 0.020 & Take from BC $\Upsilon$ mass fit \\
\hline QED/energy loss & 0.045 & Change $Q$ to $Q \times 1.46$ \\
\hline Magnetic field & 0.034 & Shift in $m_{W}$ not covered by B-field correction \\
\hline Ionizing material & 0.014 & Vary material scale factor by 0.008 \\
\hline Resolution & 0.005 & Shift between $\sigma_{b}=35 \mu \mathrm{m}$ and $\sigma_{b}=43 \mu \mathrm{m}$ \\
\hline Backgrounds & 0.005 & Vary intercept and slope parameters \\
\hline Misalignment & 0.018 & Vary $z$-scale and $\delta c$ corrections \\
\hline Trigger efficiency & 0.005 & Vary muon $p_{T}$ cut by $200 \mathrm{MeV}$ \\
\hline Fitting window & 0.005 & Vary fit window range by $\pm 20 \%$ \\
\hline$\Delta p / p$ step size & 0.003 & $\Delta p / p$ trial value step size in $\mathrm{MC}$ templates \\
\hline $\mathrm{BC}$ vs NBC & 0.075 & Halve the difference between $\mathrm{BC}$ and $\mathrm{NBC}$ \\
\hline World average & 0.027 & $\Delta m_{\Upsilon}^{\mathrm{PDG}} / m_{\Upsilon}^{\mathrm{PDG}}$ \\
\hline Total systematic & 0.101 & \\
\hline Total & 0.103 & \\
\hline
\end{tabular}

Table 8.4: Measured $\Delta p / p$ as a function of run range and instantaneous luminosity, from the beam-constrained $\Upsilon$ mass fit.

\begin{tabular}{c|c|c}
\hline \hline & Run range $1($ run $\leq 220000)$ & Run range 2 $($ run $>220000)$ \\
\hline Fit result & $(-1.185 \pm 0.028) \cdot 10^{-3}$ & $(-1.190 \pm 0.030) \cdot 10^{-3}$ \\
\hline \hline & Inst. Lum. $\leq 5.7 \cdot 10^{31}$ & Inst. Lum. $>5.7 \cdot 10^{31}$ \\
\hline Fit result & $(-1.185 \pm 0.028) \cdot 10^{-3}$ & $(-1.195 \pm 0.030) \cdot 10^{-3}$ \\
\hline \hline
\end{tabular}

\subsection{Combined Momentum Scale from $J / \psi$ and $\Upsilon$ Analyses}

We summarize the measured momentum scales from $J / \psi$, non-beam-constrained $(\mathrm{NBC}) \Upsilon$ and beam-constrained $(\mathrm{BC}) \Upsilon$ samples in Table 8.5. Since $J / \psi \rightarrow \mu \mu$ analysis is using NBC tracks, we can combine $J / \psi$ result and NBC $\Upsilon$ result first. We quote the systematic uncertainties for NBC $\Upsilon_{\mathrm{s}}$ the same as those for BC $\Upsilon_{\mathrm{s}}$ since NBC and BC cases are completely correlated. Table 8.6 lists the systematic and statistical uncertaintites. 
Table 8.5: Measured momentum scale deviations from unity

\begin{tabular}{ll}
\hline \hline & $\Delta p / p\left(\cdot 10^{-3}\right)$ \\
\hline$J / \psi \rightarrow \mu \mu$ & $-1.311 \pm 0.004_{\text {stat }} \pm 0.092_{\text {syst }}$ \\
$\Upsilon \rightarrow \mu \mu(\mathrm{NBC})$ & $-1.335 \pm 0.025_{\text {stat }} \pm 0.062_{\text {syst(excluding BC/NBC) }}$ \\
$\Upsilon \rightarrow \mu \mu(\mathrm{BC})$ & $-1.185 \pm 0.020_{\text {stat }} \pm 0.062_{\text {syst(excluding BC/NBC) }}$ \\
\hline \hline
\end{tabular}

Table 8.6: Systematic uncertainties on the momentum scale deviation from unity, derived from the non-beam-constrained $\Upsilon$ mass measurement, and the systematics that are common with the $J / \psi$ mass measurement.

\begin{tabular}{c|c|c|c}
\hline \hline Source & $J / \psi\left(\cdot 10^{-3}\right)$ & NBC- $\Upsilon\left(\cdot 10^{-3}\right)$ & common $\left(\cdot 10^{-3}\right)$ \\
\hline QED & 0.080 & 0.045 & 0.045 \\
B field non-uniformity & 0.032 & 0.034 & 0.032 \\
Ionizing material & 0.022 & 0.014 & 0.014 \\
Resolution & 0.010 & 0.005 & 0.005 \\
Backgrounds & 0.011 & 0.005 & 0.005 \\
Misalignment & 0.009 & 0.018 & 0.009 \\
Trigger efficiency & 0.004 & 0.005 & 0.004 \\
Fitting window & 0.004 & 0.005 & 0.004 \\
$\Delta p / p$ step size & 0.002 & 0.003 & 0 \\
World-average & 0.004 & 0.027 & 0 \\
\hline Total systematic & 0.092 & 0.068 & 0.058 \\
\hline Statistical & 0.004 & 0.025 & 0 \\
\hline Total & 0.092 & 0.072 & 0.058 \\
\hline \hline
\end{tabular}

Combining the results from $J / \psi$ and $\mathrm{NBC} \Upsilon$, we get

$$
\left(\frac{\Delta p}{p}\right)_{J / \psi+N B C \Upsilon}=\left(-1.329 \pm 0.004_{\mathrm{stat}} \pm 0.068_{\mathrm{syst}}\right) \cdot 10^{-3} .
$$

We take the final momentum scale to be the midpoint of the above combination and the $\mathrm{BC} \Upsilon$ result (see Table 8.5):

$$
\Delta p / p=\left(-1.257 \pm 0.004_{\text {stat }} \pm 0.068_{\text {syst }(\text { excluding } \mathrm{BC} / \mathrm{NBC})}\right) \cdot 10^{-3}
$$

where the result from BC $\Upsilon$ differs from the result from " $J / \psi+$ NBC- $\Upsilon$ " by $\sim 10 \sigma$ statistically. Adding half of the $\mathrm{BC}$ vs. NBC difference $\left(0.075 \cdot 10^{-3}\right)$ as an addi- 
tional systematic uncertainty to the Eqn. (8.11), we get the final momentum scale correction from low-mass resonances $J / \psi$ and $\Upsilon$ to be

$$
\left(\frac{\Delta p}{p}\right)_{J / \psi+\Upsilon}^{\text {low }- \text { mass }}=\left(-1.257 \pm 0.004_{\text {stat }} \pm 0.101_{\text {syst }}\right) \cdot 10^{-3}
$$

Table 8.7: Systematic uncertainties on the momentum scale from 1) the combined $J / \psi$ analysis and non-beam-constrained $\Upsilon$ analysis, and 2) the beam-constrained $\Upsilon$ analysis.

\begin{tabular}{c|c|c|c}
\hline \hline Source & $J / \psi+$ NBC- $\Upsilon\left(\cdot 10^{-3}\right)$ & BC- $\Upsilon\left(\cdot 10^{-3}\right)$ & common $\left(\cdot 10^{-3}\right)$ \\
\hline Systematic $_{\text {no BC/NBC }}$ & 0.068 & 0.068 & 0.068 \\
Statistical & 0.004 & 0.020 & 0 \\
\hline Total & 0.068 & 0.071 & 0.068 \\
\hline
\end{tabular}

\subsection{Comparison with $200 \mathrm{pb}^{-1}$ Momentum Scale}

In the previous $200 \mathrm{pb}^{-1}$ analysis at CDF, the combined $J / \psi$ and $\Upsilon$ momentum scale deviation from unity $(\Delta p / p)$ was $\left(-1.50 \pm 0.04_{\text {stat }} \pm 0.19_{\text {syst }}\right) \times 10^{-3}$. See Table 8.8 for individual result from last publication [80]. In comparison with Eqn. (8.12), we see our new momentum scale gets shifted up by $\sim 0.24 \pm 10^{-3}$ relative to the old number, which corresponds to $+20 \mathrm{MeV}$ shift in $m_{W}$ and $+22 \mathrm{MeV}$ shift in $m_{Z}$.

Table 8.8: Measured momentum scale deviations from unity from the $200 \mathrm{pb}^{-1} \mathrm{~J} / \psi$ and $\Upsilon$ analyses.

\begin{tabular}{lc}
\hline \hline & $\Delta p / p\left(\cdot 10^{-3}\right)$ \\
\hline$J / \psi \rightarrow \mu \mu$ & $-1.64 \pm 0.06_{\text {stat }} \pm 0.24_{\text {syst }}$ \\
$\Upsilon \rightarrow \mu \mu(\mathrm{NBC})$ & $-1.50 \pm 0.09_{\text {stat }} \pm 0.20_{\text {syst }}$ \\
$\Upsilon \rightarrow \mu \mu(\mathrm{BC})$ & $-1.38 \pm 0.06_{\text {stat }} \pm 0.20_{\text {syst }}$ \\
\hline \hline
\end{tabular}

We find $12 \mathrm{MeV}$ of the $22 \mathrm{MeV}$ change in $Z$ mass comes from the magnetic field non-uniformity correction, which we do apply in this new analysis but did not apply 
in $200 \mathrm{pb}^{-1}$ analysis. So this is not a true scale change but only a redefinition. Another $8 \mathrm{MeV}$ change comes from the $\mathrm{dE} / \mathrm{dx}$ model. In the old analysis we only used the Bethe-Bloch average, but now we use the full shape of Landau distribution including the tail.

As a further cross-check, we use the first $200 \mathrm{pb}^{-1}$ non-beam-constrained $\Upsilon \rightarrow \mu \mu$ events and reproduce a momentum scale which is consistent with the $200 \mathrm{pb}^{-1}$ result. See Table 8.9 and Table 8.10 for details. There Case 5 in Table 8.10 is consistent with the $200 \mathrm{pb}^{-1} \mathrm{NBC} \Upsilon$ result $\left(-1.50 \pm 0.09_{\text {stat }}\right) \times 10^{-3}$.

Table 8.9: A list of items for the study of momentum scale differences between our new result and the old $200 \mathrm{pb}^{-1}$ result by using $200 \mathrm{pb}^{-1}$ non-beam-constrained $\Upsilon \rightarrow \mu \mu$ events.

\begin{tabular}{cl}
\hline \hline Item & \multicolumn{1}{c}{ Requirement } \\
\hline 1 & $2 \mathrm{fb}^{-1}$ good run list \\
2 & $200 \mathrm{pb}^{-1}$ good run list \\
3 & B-field correction from $J / \psi$ analysis \\
4 & $2 \mathrm{fb}^{-1}:$ zScale $=1.00160$, dCurv $=0.5 \times 10^{-7}$ \\
5 & retuned background corresponding to $200 \mathrm{pb}^{-1}$ good run \\
6 & $200 \mathrm{pb}^{-1}:$ zScale $=0.99963$, dCurve $=7.0 \times 10^{-7}$ \\
\hline \hline
\end{tabular}

Table 8.10: Results of momentum scale under different cases by using $200 \mathrm{pb}^{-1}$ non-beam-constrained $\Upsilon \rightarrow \mu \mu$ events.

\begin{tabular}{lcl}
\hline \hline & $\Delta p / p$ result $\left(\times 10^{-3}\right)$ & Used Item \\
\hline Case 1 & $-1.335 \pm 0.025_{\text {stat }}$ & $1,3,4$ \\
Case 2 & $-1.270 \pm 0.083_{\text {stat }}$ & $2,3,4$ \\
Case 3 & $-1.340 \pm 0.083_{\text {stat }}$ & $2,4,5$ \\
Case 4 & $-1.495 \pm 0.083_{\text {stat }}$ & 2,5 \\
Case 5 & $-1.535 \pm 0.083_{\text {stat }}$ & $2,5,6$ \\
\hline \hline
\end{tabular}




\subsection{Cross-check of COT Momentum Scale using $Z \rightarrow \mu \mu$ Events}

We measure the $Z$ boson mass $m_{Z}$ in the $Z \rightarrow \mu \mu$ channel using the calibration of the COT tracker obtained from $J / \psi \rightarrow \mu \mu$ and $\Upsilon \rightarrow \mu \mu$ fits. At low-mass resonances $(J / \psi$ and $\Upsilon)$, we have measured a consistent value of correction scale $\alpha_{\theta}$ which is applied to the measured $\cot \theta$. For high- $p_{T}$ data like $W \rightarrow \mu \nu$ and $Z \rightarrow \mu \mu$ decays, we use $\alpha_{\theta}=1.0015 \pm 0.0002$ from the beam-constrained $\Upsilon \rightarrow \mu \mu$ measurement.

A maximum likelihood template fitting method is used to measure the $Z$ pole mass, where the fitting window is chosen to be $83.0 \mathrm{GeV}<m_{\mu \mu}<99.0 \mathrm{GeV}$. The templates are constructed using RESBOS generator to describe relevant QCD processes, convoluted with PHOTOS to make radiative photons, and DukeSim to provide the detector simulation. Since the standard RESBos $Z$ boson generation does not include the Drell-Yan and $Z / \gamma$ interference contributions, we simulate those contributions and add them with correct normalizations to the $Z$-pole distribution in the fitting stage. Before extracting the $m_{Z}$ from maximum likelihood fits, we need to determine the COT momentum resolution for high- $p_{T}$ tracks in DukeSim. The default values of SL-dependent hit resolutions are obtained from the residuals in $Z \rightarrow \mu \mu$ data (see Section 7.3). Using non-beam-constrained COT tracks, we find an empirical scale factor $h_{0}^{Z}=0.995 \pm 0.002_{\text {stat }}$ multiplying the default values in DukeSim gives the best description of the $Z \rightarrow \mu \mu$ mass lineshape from data. For this fixed $h_{0}^{Z}$ central value, we find the beamspot size $\sigma_{b}=\left(42 \pm 1_{\text {stat }}\right) \mu m$ used for beam-constrained tracks gives the best match of the width of the $Z \rightarrow \mu \mu$ mass peak. Variations of $\sigma_{b}$ by its statistical uncertainty, when propagated to $W \rightarrow \mu \nu$ mass fits, lead to $1 \mathrm{MeV}$ shift in $m_{W}$. The corresponding uncertainty on $m_{Z}$ in the $Z \rightarrow \mu \mu$ channel is found to be negligible.

The final $Z \rightarrow \mu \mu$ mass fit result is found to be:

$$
m_{Z}=\left(91180 \pm 12_{\text {stat }}\right) \mathrm{MeV}
$$




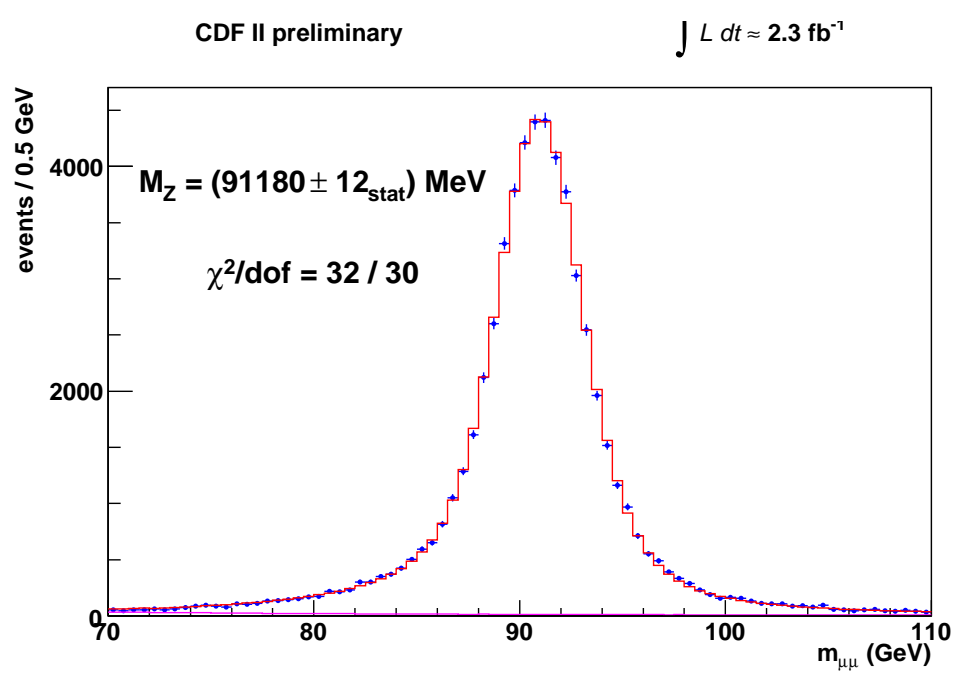

Figure 8.36: The fitted $Z \rightarrow \mu \mu$ mass using the COT momentum scale derived from $J / \psi \rightarrow \mu \mu$ and $\Upsilon \rightarrow \mu \mu$ mass fits. The fitting range is from $83.0 \mathrm{GeV}$ to 99.0 $\mathrm{GeV}$.

and is shown in Figure 8.36 where only statistical uncertainty is presented.

The systematic uncertainty on this $m_{Z}$ measurement comes from several sources: uncertainty in the COT momentum scale, the COT alignment and the uncertainty in the QED radiative corrections. Eqn. (8.12) leads to $9 \mathrm{MeV}$ on $m_{Z}$ due to COT momentum scale uncertainty. We vary $\alpha_{\theta}$ derived from beam-constrained $\Upsilon \rightarrow \mu \mu$ by its statistical uncertainty and find $2 \mathrm{MeV}$ systematic uncertainty on $m_{Z}$ due to the COT alignment. We quote the $5 \mathrm{MeV}$ difference between HORACE and PHOTOS calculations to be the uncertainty in the QED radiative corrections. This leads to a final $m_{Z}$ measurement in the dimuon channel to be:

$$
\begin{aligned}
m_{Z}^{\text {low-mass }} & =\left(91180 \pm 12_{\text {stat }} \pm 9_{\text {momentum }} \pm 2_{\text {alignment }} \pm 5_{\mathrm{QED}}\right) \mathrm{MeV} \\
& =\left(91180 \pm 16_{\text {stat+syst }}\right) \mathrm{MeV} .
\end{aligned}
$$

This result is consistent with the world-average value $m_{Z}^{\mathrm{PDG}}=91188 \pm 2 \mathrm{MeV}$ within $0.5 \sigma$. The fact that this value is consistent with world average proves that our COT momentum scale measurement is reliable. If we convert our $m_{Z}$ measurement to a 
semi-independent COT momentum relative to Eqn. (8.12), we get

$$
(\Delta p / p)^{\mathrm{Z}-\text { mass }}-(\Delta p / p)^{\text {low-mass }}=\left(-9 \pm 13_{\text {stat }} \pm 2_{\text {alignment }} \pm 5_{\mathrm{QED}}\right) \times 10^{-5}
$$

leading to the momentum scale measurement from $Z$ mass peak

$$
(\Delta p / p)^{\mathrm{Z}-\mathrm{mass}}=\left(-135 \pm 13_{\text {stat }} \pm 2_{\text {align }} \pm 5_{\mathrm{QED}}\right) \times 10^{-5}
$$

The statistical uncertainty term in Eqn. (8.16) is calculated from $\sqrt{12^{2}+2^{2}} / 91188 \approx$ $13 \times 10^{-5}$, where the $12 \mathrm{MeV}$ in this calculation is from the statistical uncertainty in Eqn. (8.15) and the $2 \mathrm{MeV}$ is from PDG $Z$ mass uncertainty. The $-9 \times 10^{-5}$ correction in the central value of momentum scale is from $(91180-91188) / 91188$. Since the $9 \mathrm{MeV}$ systematic uncertainty due to COT momentum scale is already applied in Eqn. (8.12), it is thus excluded from Eqn. (8.16). Eqn. (8.16) directly shows the consistency between the low-mass $J / \psi+\Upsilon$ calibration of the track momentum and the high-mass $Z$ boson calibration of the track momentum.

We can obtain the final COT track momentum calibration by combining the $Z$ mass fit result from Eqn. (8.16) and the $J / \psi+\Upsilon$ result from Eqn. (8.12). Since the alignment uncertainty and the QED uncertainty are correlated in both results, we take the remaining uncertainties as uncorrelated between the $Z$ mass fit and the $J / \psi$ and $\Upsilon$ mass fits (see Appendix $\mathrm{K}$ for detailed calculation). Thus the final COT track momentum from $J / \psi, \Upsilon$ and $Z$ resonances is

$$
(\Delta p / p)_{\text {final }}=\left(-129 \pm 7_{\text {uncorr }} \pm 2_{\text {align }} \pm 5_{\mathrm{QED}} \times 10^{-5}=(-129 \pm 9) \times 10^{-5}\right.
$$

which results in uncertainty of $7 \mathrm{MeV}$ on the $W$ boson mass and $8 \mathrm{MeV}$ on the $Z$ boson mass. This is a significant improvement compared with the $200 \mathrm{pb}^{-1} \mathrm{CDF}$ $m_{W}$ measurement - there the COT track momentum uncertainy on $m_{W}$ was found to be $17 \mathrm{MeV}$. 


\section{Backgrounds}

Backgrounds in the $W$ mass measurement in $W \rightarrow \mu \nu$ channel come from several sources that mimic the muon decay signature of the $W$ boson. According to the size of contributed contamination in our $W \rightarrow \mu \nu$ sample, from largest to smallest, the background processes are: $Z \rightarrow \mu \mu, W \rightarrow \tau \nu$, Kaon decay, hadronic QCD jets and cosmic rays. The $m_{T}, p_{T}$ and $\not p_{T}$ distributions of those background processes are quite different from the corresponding distributions of $W \rightarrow \mu \nu$ process. Generally, except for $Z \rightarrow \mu \mu$, those background processes have a lower mean value in $m_{T}, p_{T}$ and $p_{T}^{\prime}$, thus lead to an underestimated $W$ mass measurement if their contributions are not taken into account. It is thus important to model the amount and the shape of the individual background process. In the following sections, we will discuss each background and estimate the corresponding systematic uncertainty. The modelled backgrounds are included in our fitting templates.

\section{$9.1 \quad Z \rightarrow \mu \mu$ and $W \rightarrow \tau \nu$}

$Z \rightarrow \mu \mu$ events mimic $W \rightarrow \mu \nu$ events when one muon from $Z$ boson is not detected due to the inefficiencies and incomplete coverage of the COT. The undetected muon 
is thus mistakenly treated as a neutrino to account for substantial amount of missing energy.

$W \rightarrow \tau \nu$ events mimic the $W \rightarrow \mu \nu$ events since the $\tau$ from $W \rightarrow \tau \nu$ can futher decay into a $\mu$ and two neutrinos. The decay process of $W \rightarrow \tau \nu \rightarrow \mu \nu \nu \nu$ is topologically indistinguishable from $W \rightarrow \mu \nu$ and is suppressed by the branching ratio of $\tau \rightarrow \mu \nu \nu$ with $\operatorname{Br}(\tau \rightarrow \mu \nu \nu)=(17.36 \pm 0.05) \%$ [32].

We determine the background fractions of $Z \rightarrow \mu \mu$ and $W \rightarrow \tau \nu$ from the ratio of their acceptances to the acceptance of $W \rightarrow \mu \nu$ process.

To calculate the $Z \rightarrow \mu \mu$ acceptance, we use MC samples which are PYTHIAgenerated with the CDFSim applied. We apply our $W \rightarrow \mu \nu$ event selection criteria on the obtained $Z \rightarrow \mu \mu \mathrm{MC}$ sample and find 937,444 out of $9,345,388 \mathrm{MC}$ events pass all $W \rightarrow \mu \nu$ selection cuts. This yields an acceptance $A_{Z \rightarrow \mu \mu}=(10.031 \pm$ $\left.0.011_{M C s t a t}\right) \%$. According to the SM theory, the ratio of $W \rightarrow \mu \nu$ production cross section to $Z \rightarrow \mu \mu$ production cross section is given by $R=\frac{\sigma_{W} \cdot \operatorname{Br}(W \rightarrow \mu \nu)}{\sigma_{Z} \cdot \operatorname{Br}(Z \rightarrow \mu \mu)}=10.69 \pm$ 0.08 [78] [79] [80] at $\sqrt{s}=1.96 \mathrm{TeV}$. Tevatron Run II measurement gives $R=$ $10.84 \pm 0.15_{\text {stat }} \pm 0.14_{\text {syst }}=10.84 \pm 0.21$ [81]. The relevant experimental uncertainty is $1 \%\left(\frac{0.14_{\text {syst }}}{10.84} \approx 1 \%\right)$ on $\mathrm{R}$ due to the uncertainty on the ratio of $W$ and $Z$ boson acceptances. In our background analysis, we thus use $R=10.69 \pm 0.08 \pm 0.11_{\text {exp }}=$ $10.69 \pm 0.13$ where the experimental error term is taken to be the SM prediction value 10.69 times the experimental uncertainty $1 \%$ from Tevatron Run II measurement. After correcting the ratio $(R)$ of the $W$ to $Z$ cross sections, the $Z \rightarrow \mu \mu$ acceptance is found to be: $A_{Z \rightarrow \mu \mu}^{R}=A_{Z \rightarrow \mu \mu} / R=\left(0.938 \pm 0.011_{R}\right) \%$.

We use the same procedure to determine the $W \rightarrow \tau \nu$ acceptance. $W \rightarrow \tau \nu$ MC events are generated using PYTHIA with the CDFSim applied. We find 9,064 out of $8,143,536 W \rightarrow \tau \nu$ events pass all our $W \rightarrow \mu \nu$ selection cuts, which yields the $W \rightarrow \tau \nu$ acceptance $A_{W \rightarrow \tau \nu}=\left(0.111 \pm 0.001_{M C s t a t}\right) \%$. No additional correction 
factor is applied to this acceptance since the production cross-sections for $W \rightarrow \mu \nu$ and $W \rightarrow \tau \nu$ processes are the same in the SM.

The $W \rightarrow \mu \nu$ acceptance is obtained analogously. Similar to $Z \rightarrow \mu \mu$ and $W \rightarrow$ $\tau \nu \mathrm{MC}$ samples, the $W \rightarrow \mu \nu \mathrm{MC}$ sample is generated using PYTHIA convoluted with the CDFSim. We find 2,128,803 $W \rightarrow \mu \nu$ MC events out of 18,175,942 passing all the $W \rightarrow \mu \nu$ selection cuts, yielding an acceptance $A_{W \rightarrow \mu \nu}=\left(11.712 \pm 0.008_{M C s t a t}\right) \%$.

By calculating the ratio of $A_{Z \rightarrow \mu \mu}^{R}$ and $A_{W \rightarrow \tau \nu}$ with respect to $A_{W \rightarrow \mu \nu}$, we find a background of $Z \rightarrow \mu \mu$ to be $(7.33 \pm 0.09) \%$ and a background of $W \rightarrow \tau \nu$ to be $(0.87 \pm 0.01) \%$. See Table 9.1 for the relative acceptances for $W \rightarrow \mu \nu, Z \rightarrow \mu \mu$, $W \rightarrow \tau \nu$, and the fraction of each background in the combined acceptance.

Table 9.1: Relative acceptance of $W$ and $Z$ boson decays to pass the $W \rightarrow \mu \nu$ event selection using $\sim 2.2 \mathrm{fb}^{-1} \mathrm{MC}$ samples with some bad MC runs removed. ( $\dagger$ ) is for HEPG $Z$ mass within $(66,116) \mathrm{GeV}$. (*) Assumes other background fractions are the same as those listed in Table 9.3.

\begin{tabular}{lccc}
\hline \hline & $W \rightarrow \mu \nu \mathrm{MC}$ & $Z \rightarrow \mu \mu \mathrm{MC}$ & $W \rightarrow \tau \nu \mathrm{MC}$ \\
\hline$N_{M C}^{\text {total }}$ & 18175942 & $9345388(\dagger)$ & 8143536 \\
Passing all cuts & 2128803 & 937444 & 9064 \\
Acceptance $A$ in $\%$ & $11.712 \pm 0.008_{\text {stat }}^{M C}$ & $10.031 \pm 0.011_{\text {stat }}^{M C}$ & $0.111 \pm 0.001_{\text {stat }}^{M C}$ \\
R corrected acceptance & & $0.938 \pm 0.011_{R}$ & \\
Acceptance fraction & $91.775 \pm 0.122_{\text {stat }}^{M C}$ & $7.353 \pm 0.090_{R}$ & $0.872 \pm 0.009_{\text {stat }}^{M C}$ \\
\hline Background in \% $\left(^{*}\right)$ & & $7.331 \pm 0.090_{R}$ & $0.869 \pm 0.009$ \\
\hline \hline
\end{tabular}

Further studies have been carried out to tune the CDFSim as we have found there are noticeable mismatches in the recoil response distribution and recoil resolution distribution between data and CDFSim [82]. CDFSim also needs to be tuned as we find it does not describe the muon energy deposition in the plug calorimeter observed in data. This descrepancy needs to be corrected as the energy deposition of plug muon will enter the recoil energy calculation and bias the $m_{W}$ background measurement. 
The data-CDFSim disagreement in recoil response is reduced by applying a scale factor of $95.5 \%$ to all tower energies in the CDFSim to minimize the $\chi^{2}$ between data and CDFSim using $p_{T}$-balance plot from $Z \rightarrow \mu \mu$ events. We improve the agreement between data and CDFSim recoil resolution by removing high luminosity runs (> 222418) in the Electroweak CDFSim samples. Using $Z \rightarrow \mu \mu$ control sample, we find the data-CDFSim inconsistency in the muon energy deposition in the plug calorimeter, in terms of EM energy distribution and hadronic energy distribution, gets removed by adding a constant to the measured tower energy in the CDFSim.

With these corrections applied to CDFSim sample and following the same procedure discussed before, we obtain the background fraction of $Z \rightarrow \mu \mu$ and $W \rightarrow \tau \nu$ to be $\left(7.348 \pm 0.088_{\text {stat }}\right) \%$ and $\left(0.875 \pm 0.009_{\text {stat }}\right) \%$, respectively.

\subsection{1 $Z \rightarrow \mu \mu$ systematic uncertainties}

The systematic uncertainties of $Z \rightarrow \mu \mu$ background normalization come from several sources. The fractional uncertainties of background normalization from $R$ value uncertainty (see discussion before), recoil response tuning, recoil resolution tuning and energy deposition tuning of muons in plug calorimeter (MIP) are found to be $1.2 \%, 0.3 \%, 0.5 \%$ and $0.2 \%$, leading to a total fractional uncertainty of $1.4 \%$. The systematic uncertainty due to $R$ is already shown previously. We will discuss the remaining three systematic uncertainties as follows.

The systematic uncertainty from recoil response tuning is obtained by varying the scale of $95.5 \%$ up and down by $1.5 \%$, making the average $\chi^{2}$ to vary about minimum by +9 . We treat the variations as symmetrized $\pm 3 \sigma$ uncertainty on the CDFSim tower energy scale from response tuning.

The noticeable data-CDFSim mismatch in the recoil resolution is mainly caused by the underlying event description in CDFSim. To quantify the systematic uncertainty caused by underlying event, we use different luminosity runs to study the level 
of CDFSim-data disagreement. We find the agreement gets improved by removing the Electroweak group's CDFSim samples for high luminosity runs (> 222418). The uncertainy is estimated using MC runs $(<212133)$ to reduce the recoil resolution, and using MC runs (> 228596) to increase the recoil resolution.

The uncertainty due to MIP tuning is obtained by selecting a different sample of $Z \rightarrow \mu \mu$ events with one muon in the plug detector by widening the mass range from default $81-101 \mathrm{GeV}$ to $66-116 \mathrm{GeV}$. A new correction is found to match data and CDFSim in EM energy distribution and hadronic energy distribution. The change caused by this new correction relative to the default correction is quoted as MIP tuning systematic uncertainty.

The final $Z \rightarrow \mu \mu$ background normalization is thus $\left(7.348 \pm 0.094_{\text {syst }+ \text { stat }}\right) \%$. Its uncertainty on $m_{W}$ is found to be $1.4 \mathrm{MeV}, 3.6 \mathrm{MeV}$ and $0.7 \mathrm{MeV}$ for $m_{T}, p_{T}^{\mu}$ and $p_{T}^{\nu}$ fits.

The variation on $Z \rightarrow \mu \mu$ background shapes due to recoil response tuning, recoil resolution tuning and MIP tuning shifts $m_{W}$ by $(1.2,1.2,1.2) \mathrm{MeV},(0.7,0.7,1.5)$ $\mathrm{MeV}$ and $(1.0,1.0,5.0) \mathrm{MeV}$ on $\left(m_{T}, p_{T}^{\mu}, p_{T}^{\nu}\right)$ fits, respectively. The combined shape systematic uncertainty on $m_{W}$ is found to be $1.7 \mathrm{MeV}, 1.7 \mathrm{MeV}$ and $5.4 \mathrm{MeV}$ in $m_{T}$, $p_{T}^{\mu}$ and $p_{T}^{\nu}$ fits.

\subsection{2 $W \rightarrow \tau \nu$ systematic uncertainty}

For $W \rightarrow \tau \nu$ background normalization, we perform an independent study by using DukeSim. The $W \rightarrow \tau \nu$ kinematics, radiative corrections, $\tau \rightarrow \mu \bar{\nu} \nu$ branching ratio and decay spectrum, and $\tau$ polarization are incorporated into DukeSim. We find the DukeSim prediction is in excellent agreement with CDFSim prediction. Since DukeSim gives negligible MC statistical error, we quote $0.880 \%$ with zero statistical error.

The main source of the systematic uncertainty on $W \rightarrow \tau \nu$ background normal- 
ization is the hadronic recoil model. We find the fractional systematic uncertainty to be $0.05 \%$ [82]. This leads to the final $W \rightarrow \tau \nu$ background normalization to be $\left(0.880 \pm 0.004_{\text {syst+stat }}\right) \%$. The uncertainties on $m_{W}$ due to the shape of $m_{T}, p_{T}^{\mu}$ and $p_{T}^{\nu}$ spectra from $W \rightarrow \tau \nu$ events are negligible.

The final numbers of $Z \rightarrow \mu \mu$ and $W \rightarrow \tau \nu$ background normalization, together with normalization and shape systematic uncertainties on $m_{W}$, are summarized in Table 9.3 .

\subsection{Decay in Flight}

Long-lived particle decaying into a muon is another source of background to the $W$ boson mass measurement in the muon channel. The dominant source of "decay-inflight" (DIF) background comes from kaons and pions decaying into muons before reaching the calorimeter.

Previous study shows a DIF background of $\left(0.3 \pm 0.2_{\text {stat }}\right) \%$ [8] by using control sample from $d_{0}$ sideband $\left(0.3<\left|d_{0}\right|<0.6\right)$. For this round $m_{W}$ analysis, an additional two-dimensional transitions cut is applied to reduce DIF background. The number of transitions $N_{\text {trans }}$ counts how many times the hit residuals change sign. A regular track is expected to have hits randomly switching from one side to the other, while a DIF track tends to have many consecutive hits on one side, followed by many consecutive hits on the other side of the track, thus leading to generally smaller $N_{\text {trans }}$ and poor $\chi^{2} / d$.f.. This characteristic motivates us to study the relationship between track $\chi^{2} / d$.f. and $N_{\text {trans }}$ the track has experienced, using a signal sample of $Z \rightarrow \mu \mu$ data and a DIF control sample (see Figure 9.2 for illustration). From Figure 9.2 we can see $N_{\text {trans }}>30+2 \cdot \chi^{2} /$ d.f. can remove a significant amount of backgrounds.

We estimate the normalization of DIF background through $\chi^{2}$ minimization by using the tail region of track- $\chi^{2} / d$.f. distribution. The signal template of track$\chi^{2} / d$.f. is obtained from $Z \rightarrow \mu \mu$ data. The template for DIF tracks is obtained 


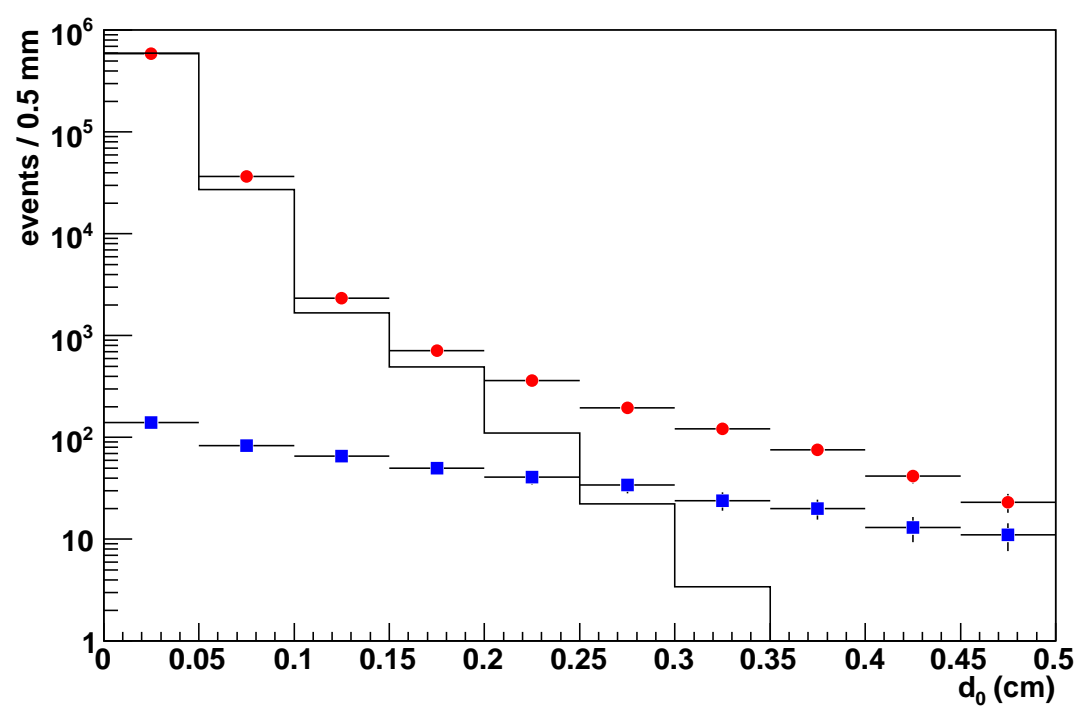

Figure 9.1: Comparison of the impact parameter $d_{0}$ distributions. The red points are for the $W$ boson candidate sample, the black histrogram is for $W$ boson simulation from DukeSim, and the blue points are for a DIF-enriched data sample obtained by anti-selecting on the $N_{\text {trans }}$. We use $0.2<d<0.5 \mathrm{~cm}$ to obtain DIF sample.

from the standard $W \rightarrow \mu \nu$ selection with $d_{0}$ cut changed to $2<d_{0}<5 \mathrm{~mm}$, as the $d_{0}$ sideband region contains many mis-reconstructed tracks and is thus DIFenriched. Figure 9.1 shows the $d_{0}$ distribution for the $W$ boson data sample, $W$ boson simulated sample from DukeSim and a DIF-enriched data sample by reversing the selection cut on the $N_{\text {trans. }}$. We can see that $d_{0}<1 \mathrm{~mm}$ contains almost all prompt muons, while $2<d_{0}<5 \mathrm{~mm}$ is DIF-dominated. The sum of signal template and floated normalization of DIF template, is fitted to the track- $\chi^{2} / d . f$. of the $W \rightarrow \mu \nu$ events. Using track- $\chi^{2} / d$.f. distribution up to 5.0, we find the best-fit DIF background normalization to be $\left(0.47 \pm 0.07_{\text {stat }}\right) \%$.

Since our default selection is $\chi^{2} / d . f .<3$, we need to correct the previously obtained normalization. We find the correction factor to be $(84 \pm 1) \%$. Since some real $W \rightarrow \mu \nu$ events can have large $d_{0}$ due to resolution, our DIF background template is 
thus not pure and also needs to be corrected. We use $Z \rightarrow \mu \mu$ data events to estimate the number of $W \rightarrow \mu \nu$ events in the $d_{0}$ sideband. Propagating this correction as well as its statistical error leads to the final DIF background fraction in our $W \rightarrow \mu \nu$ sample to be $\left(0.24 \pm 0.08_{\text {stat }}\right) \%$. As a cross-check, we repeat the above computation by further splitting the $d_{0}$ sideband sample into $2<d_{0}<3 \mathrm{~mm}$ subsample and $3<$ $d_{0}<5 \mathrm{~mm}$ subsample. The corresponding result is found to be $\left(0.26 \pm 0.10_{\text {stat }}\right) \%$ and $\left(0.18 \pm 0.14_{\text {stat }}\right) \%$, respectively. Since cross-check results are statistically consistent, we use the statistically more accurate number of $\left(0.24 \pm 0.08_{\text {stat }}\right) \%$ from the $2<d_{0}<5$ $\mathrm{mm}$ sideband as the final DIF background fraction in our $W \rightarrow \mu \nu$ selection. Its statistical uncertainty, when propagated to $m_{W}$, is found to be $0.1 \mathrm{MeV}, 1.3 \mathrm{MeV}$ and $0.0 \mathrm{MeV}$ for the $m_{T}, p_{T}^{\mu}$ and $p_{T}^{\prime}$ respectively.

We obtain the default DIF background spectra for $m_{T}, p_{T}^{\mu}$ and $\not p_{T}$ fits by requiring $2<d_{0}<5 \mathrm{~mm}$ and $N_{\text {trans }}<30+2 \cdot \chi^{2} / d$.f. while keeping all the other cuts identical to the standard $W \rightarrow \mu \nu$ event selection. To obtain the systematic uncertainties due to the shape of DIF background, we generate different DIF background spectra by using combinations of cuts on $d_{0}$ sideband and the 2-D $N_{\text {trans }}{ }^{2} \chi^{2} / d f$. The resulting shape systematic uncertainties on $m_{W}$ is estimated to be $0.9 \mathrm{MeV}, 2.8 \mathrm{MeV}$ and 1.4 $\mathrm{MeV}$ for the $m_{T}, p_{T}^{\mu}$ and $\not p_{T}^{\prime}$ fits respectively.

\subsection{QCD Jets}

Another background is the QCD hadronic jets where one jet is mistakenly identified as a muon track. We use JETNET package with a ROOT-interface [83], also known as RoOT-JETnET ${ }^{1}$, to separate QCD jet background from signal. The JETnET is a FORTRAN collection of routines to train and test artifical neural networks (NN). It reads in master learning files to train the NN by minimizing a loss function. The best-trained NN can then be applied to observations to infer the information we

\footnotetext{
1 The Root-JETnET package can be obtained from the CDF offline CVS repository.
} 


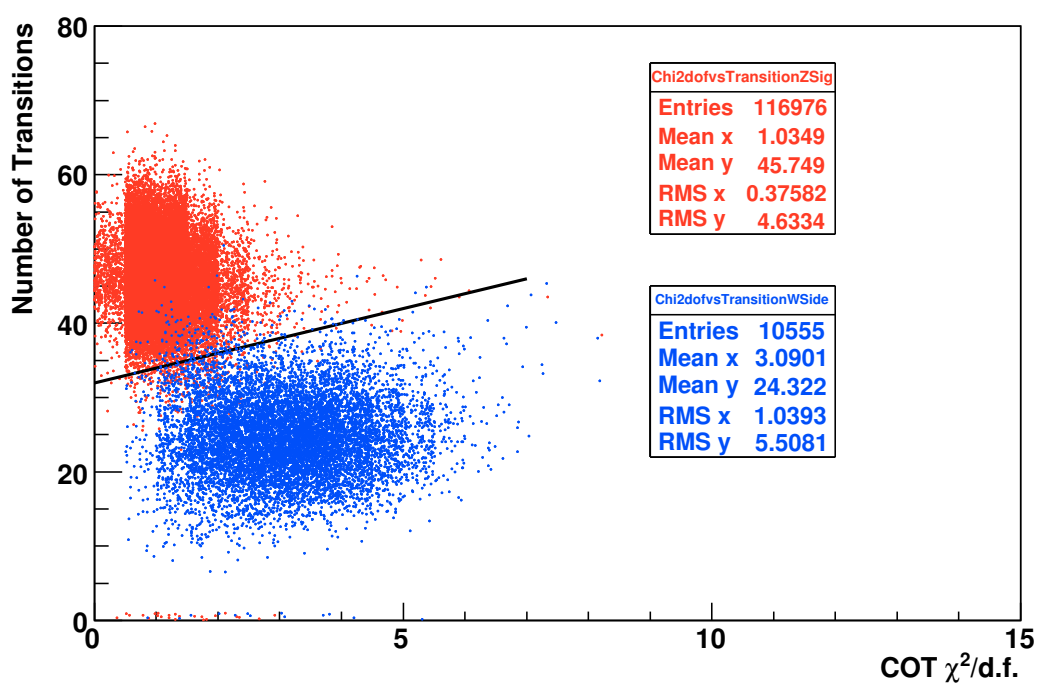

Figure 9.2: Number of transitions $N_{\text {trans }}$ as a function of $\chi^{2} / d f$ for $Z \rightarrow \mu \mu$ data signal (red points) and DIF muons from $W$ sideband (blue points). The black line is $N_{\text {trans }}=30+2 \cdot \chi^{2} / d f$.

want. Details of JeTnet can be found in reference [84].

Our goal is to combine signal and background muon spectra from $\mathrm{NN}$ in a way such that the combined spectrum matches the $W$ muon spectrum from the same NN. The background fraction varies as a free parameter and can thus be extracted by $\chi^{2}$-minimization. To fulfill this goal, we select $W$ muons from $W \rightarrow \mu \nu \mathrm{MC}$ samples as signal file and anti-select $W$ muons from data as background file by requiring $p_{T}^{\nu}<10 \mathrm{GeV}$ and $u_{T}<45 \mathrm{GeV}$. Two muon identifcation variables, track isolation fraction (trkIso) ${ }^{2}$ and hadronic calorimeter isolation (hadIso), are chosen for NN training and testing. These two variables not only provide the most discrimination power between signal muons and fake muons, but also are relatively insensitive to QED radiations which will spoil the EM isolation.

The distributions of isolation energy are highly peaked around zero and highly skewed with a discontinuity just above zero, followed by a long tail towards positive

\footnotetext{
${ }^{2}$ In the calculation of trkIso, we require $\eta$ - $\phi$ cone size $\Delta R<0.4$, the central track $p_{T}>0.5 \mathrm{GeV}$. $f_{\text {trkIso }}=\operatorname{trkIso} / \mathrm{p}_{\mathrm{T}}$.
} 
values. These features make them unsuitable as inputs to JETnET. To make lessskewed and smooth distributions, we apply a small shift and gaussian smear to those two variables, then use their logarithmic forms as inputs to the NN. Information loss due to these transformations are negligible.

The NN analysis is performed for CMUP-type muons and CMX-type muons separately since the corresponding fraction of QCD jet background may be different. We use a three-layer structure, as shown in Figure 9.3, to train and test the NN.

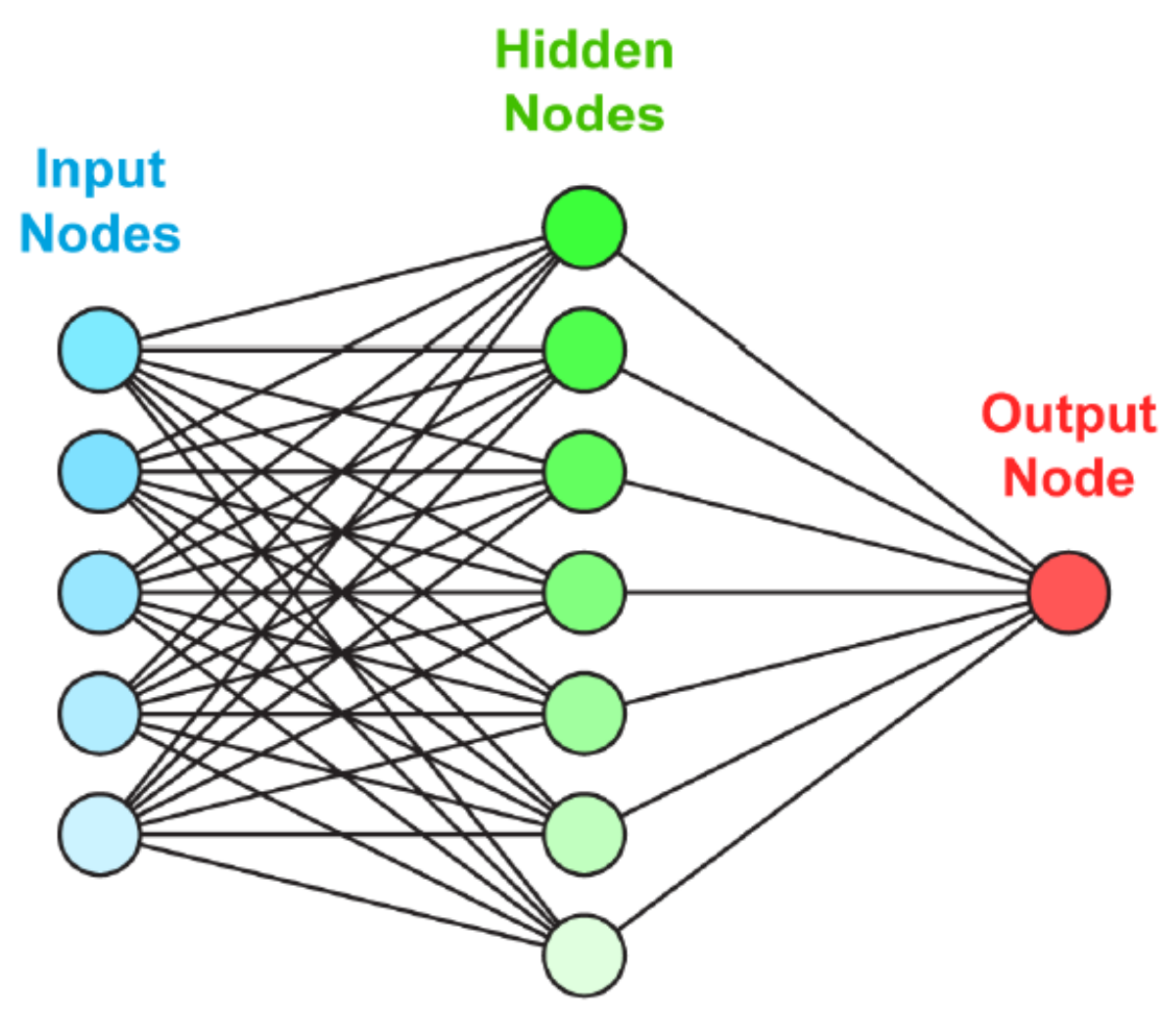

FIGURE 9.3: An illustration of the three-layer structure used in the QCD jet background NN analysis.

We first use $Z$ muons as control sample since we know the QCD jet background in $Z$ muons is consistent with 0 , given that we observe no same-sign dimuon events 
passing our selection. The log distributions of gaussian-smeared trkIso and hadIso for $Z$ data and MC muons of CMUP type are shown in Figure 9.4. To match the mean and RMS of the corresponding data distributions, we apply a small shift and a scale factor to CDFSim variable $\log$ (hadIso). Figure 9.5 compares $Z \mathrm{MC}$ signal muon with $W$ data QCD jets, which are inputs for ROOT-JETNET after those transformations. After NN training, we apply the obtained training weights to the $Z$ CMUP muons from data. In this way, we obtain three basic NN-out distributions as shown in Figure 9.6 a. We find that a "consistent-with-zero" QCD jet background in the $Z$ boson data for CMUP muons - $\left(0.06 \pm 0.06_{\text {stat }}\right) \%$ - minimizes the $\chi^{2}$ (see Figure 9.6 c). This procedure shows the NN method is robust.

As a second step, we apply the same trained NN to $W$ muons with the signal file replaced by $W$ CMUP-muons from $W \rightarrow \mu \nu$ MC samples, and the test file replaced by $W$ CMUP-muons from $W$ data while the QCD background control sample is fixed. The same transformations and tunings are also applied to the CDFSim variable $\log$ (hadIso) to match the mean and RMS of the corresponding data distribution. We find $\left(0.01 \pm 0.02_{\text {stat }}\right) \%$ QCD background for $W$ CMUP-muons. The corresponding plots are shown in Figure $9.6 \mathrm{~b}$ and Figure $9.6 \mathrm{~d}$. The variation of fitted $\chi^{2}$ as a function of the QCD jet background fraction is shown in Figure 9.7 for $Z$ CMUPmuons and $W$ CMUP-muons, respectively. Using the same strategy, we find the QCD background in $W$ CMX-muons is $\left(0.06 \pm 0.02_{\text {stat }}\right) \%$ (see Appendix $\mathrm{H}$ for details).

As a cross-check, we perform the whole process in two alternative ways. The first way is to use trkIso information only. The second way is to use trkIso and hadIso with the tower energy threshold raised from $0 \mathrm{MeV}$ to $200 \mathrm{MeV}$ in hadIso calculation. This threshold changes the shape of the CDFSim distribution of $\log$ (hadIso) to become more similar to the data $\log$ (hadIso) distribution. This allows us to study the systematic uncertainty from the hadIso modelling. Both cross-checks, within a variability of $0.02 \%$ in the results, suggest a "consistent-with-zero" QCD background in 
CMUP-muons while a tiny fraction $(\sim 0.06 \%)$ in CMX-muons. We quote the $0.02 \%$ as the systematic uncertainty in the modelling of isolation energy deposited in the hadronic calorimeter, which will affect the QCD jet background estimation in both the CMUP-muons and the CMX-muons. Since the fractions of $W$ boson data in the CMUP and CMX are almost equal, we average the two results from CMUP-muons and CMX-muons to a single value $\left(0.035 \pm 0.014_{\text {stat }} \pm 0.020_{\text {syst }}\right) \%=(0.035 \pm 0.025) \%$. We find the systematic uncertainty on $m_{W}$ due to QCD background normalization uncertainty is $0,0.5$ and $1 \mathrm{MeV}$. The respective results for CMUP-muons and CMXmuons are summarized in Table 9.2.

We obtain the QCD background spectrum from data by keeping the kinematic selection the same as the $W \rightarrow \mu \nu$ selection (see Table 5.4), while reversing the muon identification criteria $E_{E M}, E_{H a d}$ and track-stub matching cut $\Delta x$, and reversing the isolation cut by requiring isolation $>0.25$. All the other cuts are kept the same as standard selection (see Table 5.2). The shape uncertainty is estimated by antiselecting different combinations of these identification cuts [8]. The shape systematic uncertainty on $m_{W}$ is found to be $(0.5,0.5,0.3) \mathrm{MeV}$ on $\left(m_{T}, p_{T}, \not p_{T}\right)$ fits.

Table 9.2: QCD background in CMUP-muons and CMX-muons.

\begin{tabular}{lcc}
\hline \hline Muon Type & Size $(\%)$ & $\chi^{2} / \mathrm{df}$ \\
\hline$Z$ CMUP-muon & $0.06 \pm 0.06$ & $21 / 29$ \\
$W$ CMUP-muon & $0.01 \pm 0.02$ & $34 / 29$ \\
$Z$ CMX-muon & $-0.03 \pm 0.05$ & $25 / 19$ \\
$W$ CMX-muon & $0.06 \pm 0.02$ & $31 / 15$ \\
\hline \hline
\end{tabular}

\subsection{Cosmic Background}

Most of the cosmic ray events are removed by a specially designed algorithm (cosmic tagger). The remaining background is estimated by using the $t_{0}$ distributions for $W$ 

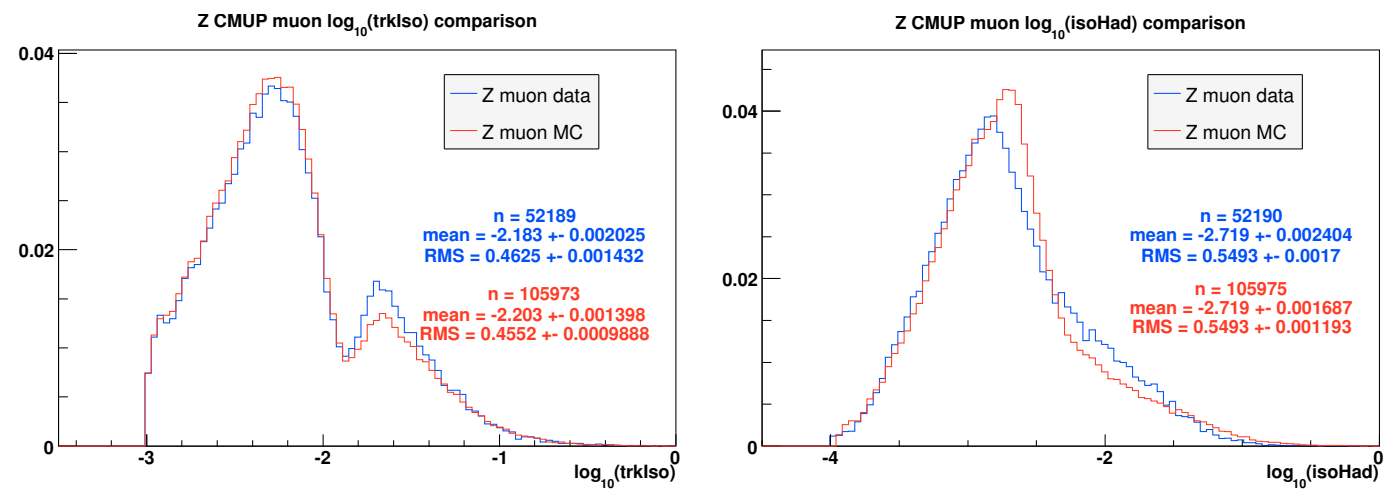

Figure 9.4: The log distributions of trkIso (left) and hadIso (right) for $Z$ muons of CMUP type. The red histogram is for $Z$ CMUP muons from MC while the blue histogram is for $Z$ CMUP muons from data. The MC spectrum in the right plot has been linearly transformed to match mean and rms of the data spectrum.
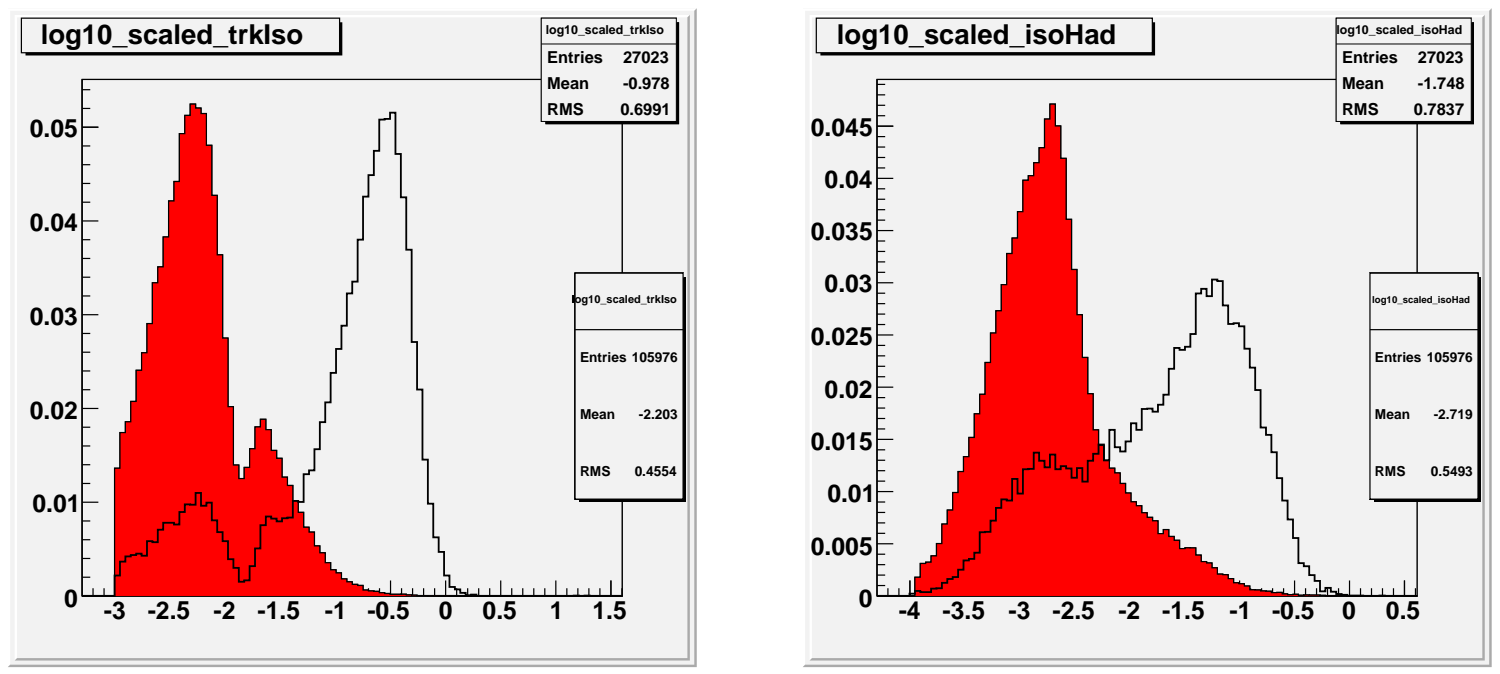

Figure 9.5: The log distributions of trkIso (left) and hadIso (right) for $Z$ muons of CMUP type. The red histogram is for $Z \mathrm{MC}$ muon while the black histogram is for QCD jet events from $W$ boson candidates with $p_{T}^{\nu}<10 \mathrm{GeV}$ and $u_{T}<45 \mathrm{GeV}$. 


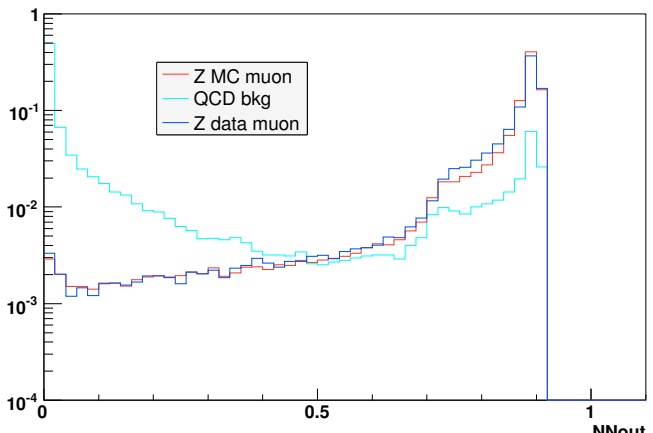

(a) Z CMUP-muon NN output

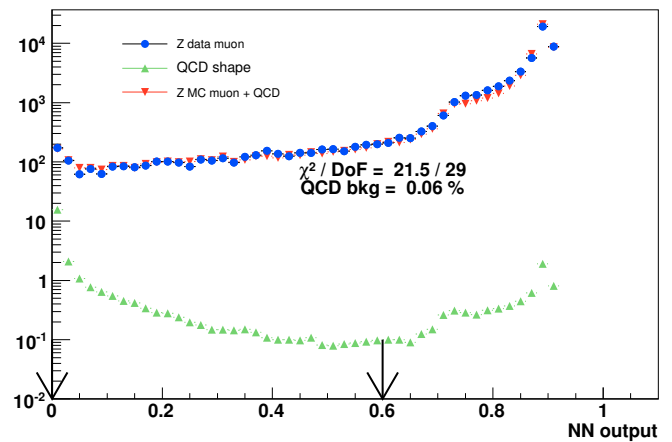

(c) Best $\chi^{2}$ fit for $Z$ CMUP-muon NN out

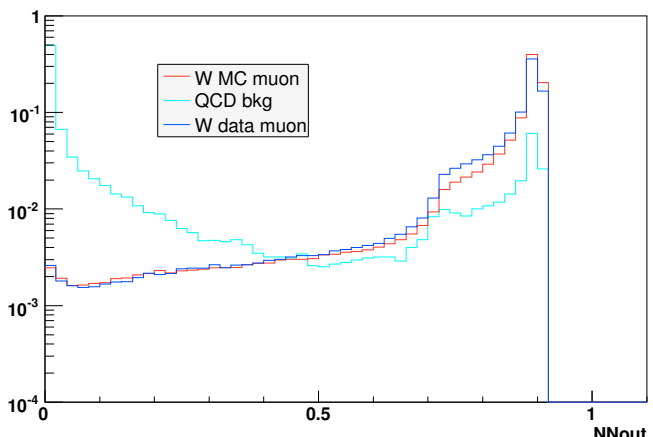

(b) $W$ CMUP-muon NN output

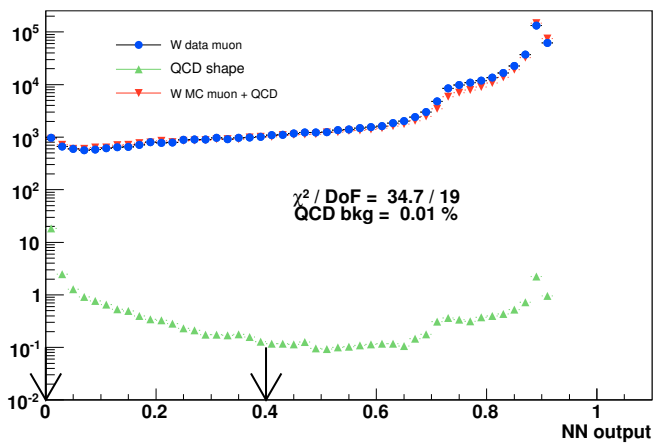

(d) Best $\chi^{2}$ fit for $W$ CMUP-muon NN out

FiguRE 9.6: The NN output distributions for $Z$ MC CMUP-muons, $Z$ data CMUPmuons and QCD jet events (left), and $W$ MC CMUP-muons, $W$ data CMUP-muons and QCD control sample (right).
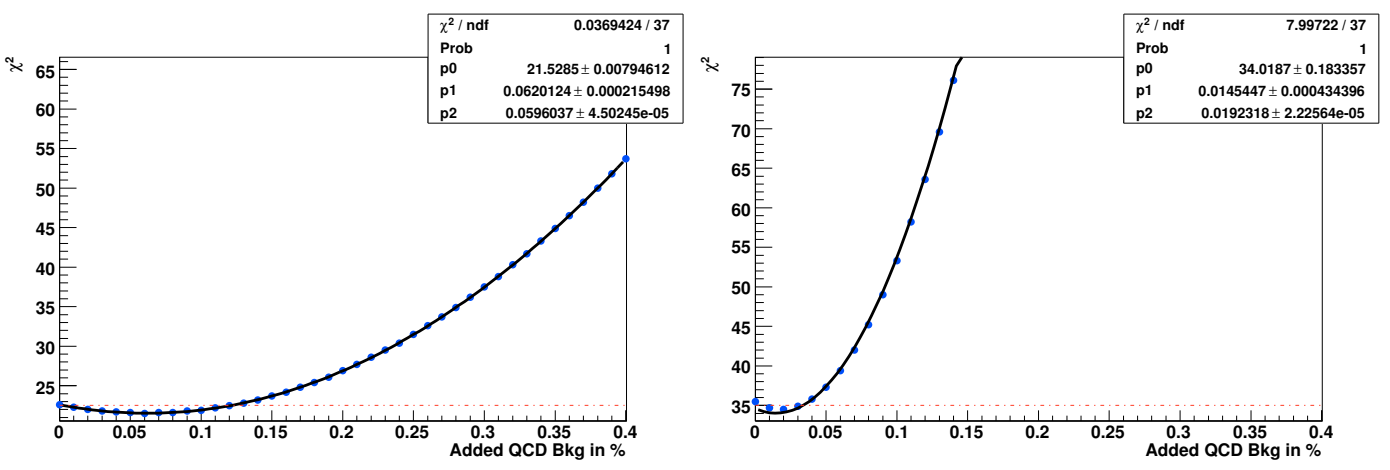

FiguRE 9.7: $\chi^{2}$ as a function of the fraction of QCD jet background for $Z$ CMUP muons (left) and $W$ CMUP muons (right). 
events, $Z$ events and comic ray events. There $t_{0}$ is the time between the $p \bar{p}$ bunch crossing and the muon's production. The $t_{0}$ distribution for cosmics is expected to be reasonably flat around 0 (the beam-crossing time) since it passing through the detector continuously in time. On the contrary, the $t_{0}$ distribution from $W$ events tend to peak around 0 . We normalize sideband region from cosmic $t_{0}$ distribution to the corresponding region from $W t_{0}$ distribution and count the number of cosmic ray events that fall within the signal range. A fraction of $(0.05 \pm 0.05) \%$ cosmic ray background was found in $200 \mathrm{pb}^{-1} W$ mass measurement [8]. In the $2.2 \mathrm{fb}^{-1}$, we scale that number by the ratio of run time to integrated luminosity and estimate the background fraction to be $(0.02 \pm 0.02) \%^{3}$. We use the cosmic-ray background shape as in the $200 \mathrm{pb}^{-1}$ analysis, which was obtained by using cosmic-ray tagger. Using the same method as discussed before, we find the systematic uncertainties due to normalization (shape) uncertainties to be $0.5,1.0,0.5 \mathrm{MeV}(0,0,0.5 \mathrm{MeV})$ on the $m_{T}, p_{T}^{\mu}$ and $p_{T}^{\prime}$, respectively.

\subsection{Background Systematic Uncertainties on $m_{W}$}

The backgrounds in $m_{T}, p_{T}^{\mu}$ and $p_{T}^{\prime}$ distributions are shown in Figure 9.8, Figure 9.9 and Figure 9.10.

To obtain the systematic uncertainties on $m_{W}$ arising from backgrounds, we generate high statistics MC template and data-like MC file using the fast detector simulation (DukeSim). Background sizes are varied by $\pm 3 \sigma$ when fitting the data-like MC file against the $\mathrm{MC}$ templates. The shifts in fitted $m_{W}$ are converted into $1 \sigma$ uncertainty assuming linear relationship. The uncertainties due to background kinematic shapes are estimated by using different background distributions [80] [82]. The sys-

3 The $200 \mathrm{pb}^{-1}$ data used in previous CDF analysis were taken between Feb., 2002 and Aug., 2003. The $2.2 \mathrm{fb}^{-1}$ data used in this analysis were taken between Feb., 2002 and Aug., 2007. Thus the ratio of run-time to integrated luminosity is $\frac{5.5 \mathrm{yr} / 1.5 \mathrm{yr}}{2.2 \mathrm{fb}^{-1} / 0.2 \mathrm{fb}^{-1}} \approx 0.33$ 


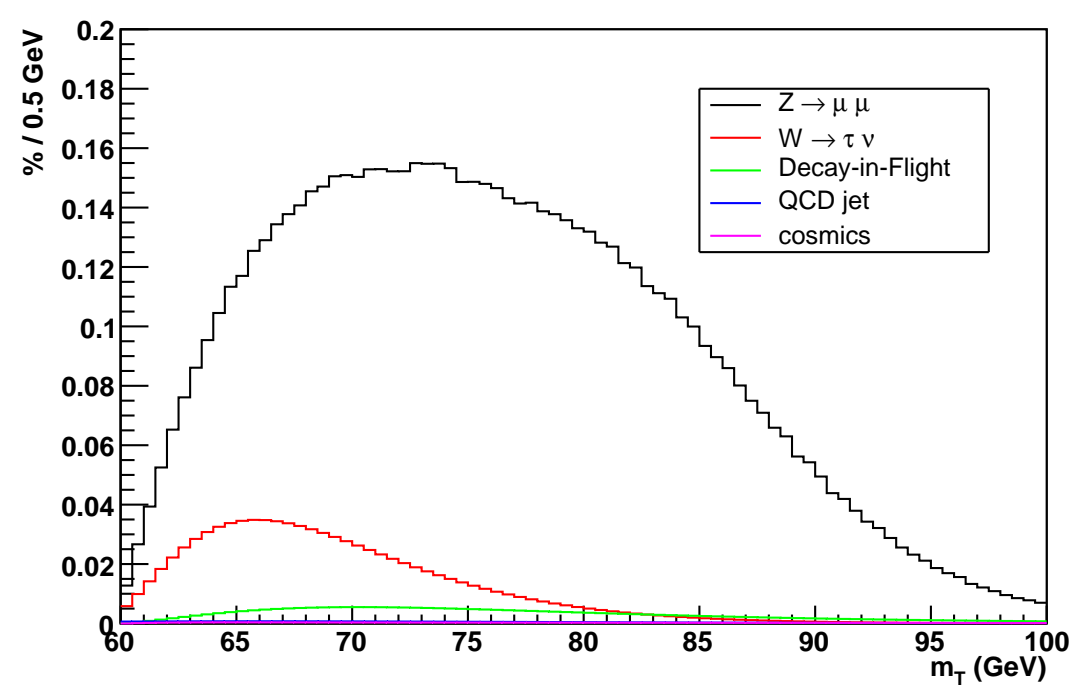

FiguRE 9.8: $m_{T}$ distributions for all backgrounds in $W \rightarrow \mu \nu$ channel. Each background is normalized to its corresponding fraction in Table 9.3.

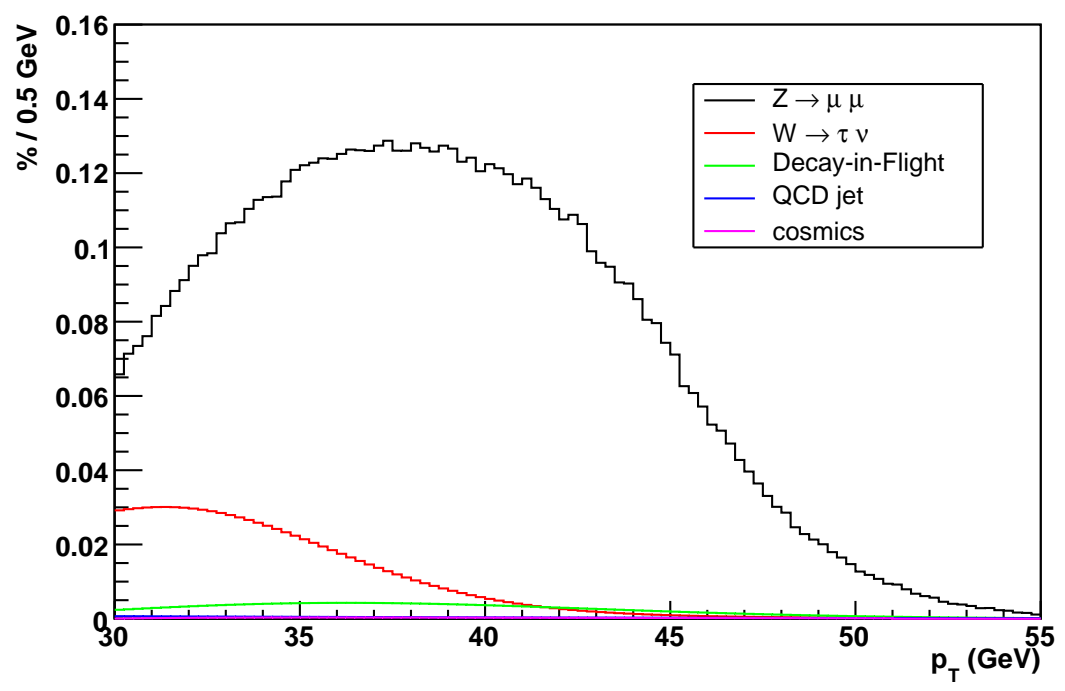

Figure 9.9: $p_{T}$ distribtions for all backgrounds in $W \rightarrow \mu \nu$ channel. Each background is normalized to its corresponding fraction in Table 9.3. 


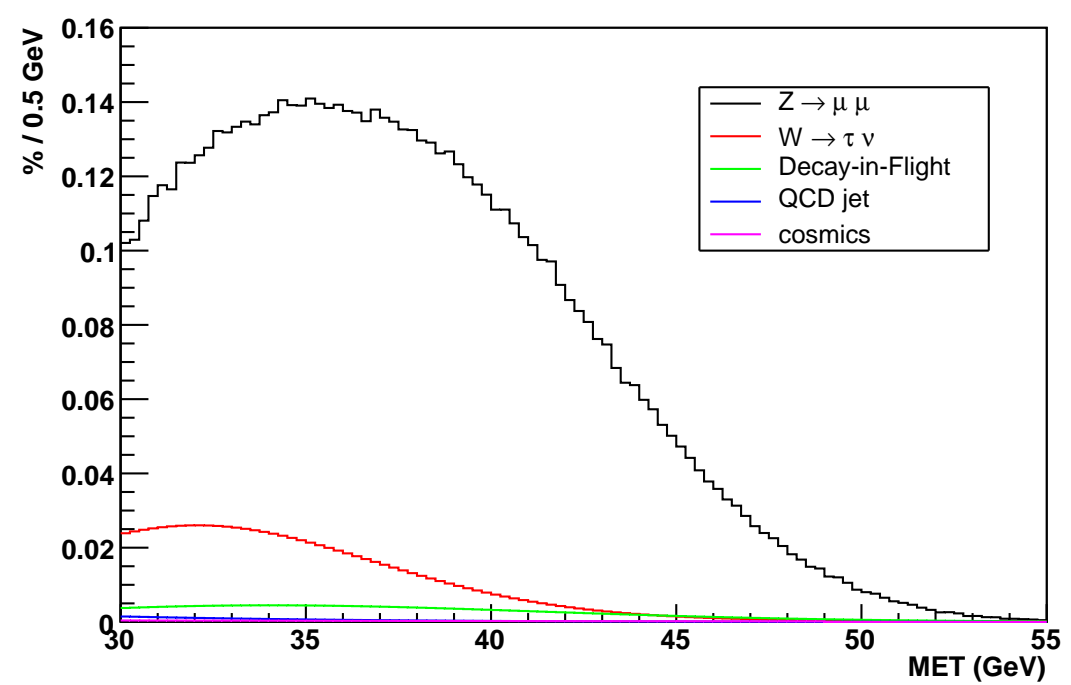

FIGURE 9.10: $\not p_{T}$ distribtions for all backgrounds in $W \rightarrow \mu \nu$ channel. Each background is normalized to its corresponding fraction in Table 9.3.

tematic uncertainties due to background size and background shape, converted into $1 \sigma$, are summarized in Table 9.3. These uncertainties reflect a significant reduction from the uncertainties obtained with $200 \mathrm{pb}^{-1}$ CDF Run II data [8].

Table 9.3: Systematic uncertainties on $m_{W}$ due to $W \rightarrow \mu \nu$ background size and shape uncertainties.

\begin{tabular}{lcccc}
\hline & \multicolumn{1}{c}{$\%$ of } & \multicolumn{3}{c}{$\delta m_{W}$ in size (shape) } \\
Background & $W \rightarrow \mu \nu$ data & $m_{T}$ fit & $p_{T}^{\mu}$ fit & $p_{T}^{\nu}$ fit \\
\hline$Z \rightarrow \mu \mu$ & $7.35 \pm 0.09$ & $1.4(1.7)$ & $3.6(1.7)$ & $0.7(5.4)$ \\
$W \rightarrow \tau \nu$ & $0.880 \pm 0.004$ & $0.2(0.0)$ & $0.2(0.0)$ & $0.2(0.0)$ \\
DIF & $0.24 \pm 0.08$ & $0.1(0.9)$ & $1.3(2.8)$ & $0.0(1.4)$ \\
QCD & $0.035 \pm 0.025$ & $0.0(0.5)$ & $0.5(0.5)$ & $1.0(0.3)$ \\
Cosmic Rays & $0.02 \pm 0.02$ & $0.5(0.0)$ & $1.0(0.0)$ & $0.5(0.5)$ \\
\hline Combined & & $1.5(2.0)$ & $4.0(3.3)$ & $1.3(5.6)$ \\
\hline Total (size + shape) & & 2.5 & 5.2 & 5.8 \\
\hline \hline
\end{tabular}




\section{Hadronic Recoil Model}

We refer all the particles recoiling against the produced $W$ or $Z$ bosons as the recoil. The modelling of the recoil affects the kinematic distributions of $m_{T}, p_{T}^{\mu}$ and $p_{T}^{\nu}$ and thus influences the extracted $m_{W}$. A precise $m_{W}$ measurement requires an accurately modelled calorimeter response and resolution to the particles recoiling against the $W$ boson.

There are three physics processes contribute to the recoil: gluon radiation in the $W$ production, underlying energy from the spectator partons as well as additional $p \bar{p}$ collisions, and photon radiation. The first one arises from the initial state gluon radiated from the quarks that produce $W / Z$. The underlying energy consists of two parts: the energy associated with the remnants of the $p \bar{p}$ collision from which a $W / Z$ is produced, and the energy from multiple $p \bar{p}$ interactions in a given bunch crossing. Photons emitted by charged lepton which are not in the excluded region also contribute to the recoil calculation. In our analysis, we use $Z \rightarrow \mu \mu$ events to constrain the recoil model for $W$ since $W$ and $Z$ bosons are close in mass and have a common production mechanism.

In this chapter, we describe the modelling of the recoil by using $Z \rightarrow \mu \mu$ events 
and present the tuning results using the fast simulation. A schematic diagram of the recoil momentum vector $\vec{u}_{T}$ in a $W$ boson event is shown in Figure 10.1. $\vec{u}_{T}$ is decomposed into components parallel $\left(u_{\|}\right)$and perpendicular $\left(u_{\perp}\right)$ to the direction of the muon from $W$ boson decay. The $W$ boson mass is sensitive to bias in $u_{\|}$since it affects $m_{T}$ and $p_{T}^{\nu}$ spectra directly through recoil measurements. The $p_{T}^{\mu}$ spectrum is however, less sensitive to $u_{\|}$as the calculation of $p_{T}^{\mu}$ does not use the measured recoil. The $u_{\perp}$ component is sensitive to the calorimeter resolution.

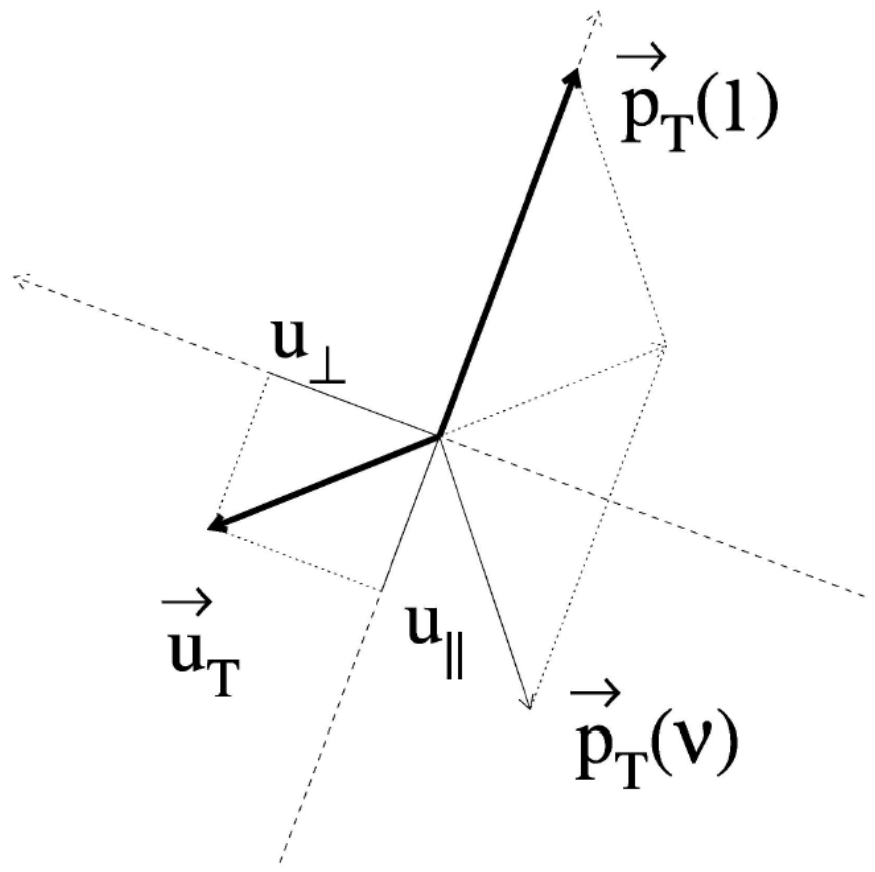

FigURE 10.1: Illustration of $u_{\|}$and $u_{\perp}$ for the decomposition of the recoil momentum $\vec{u}_{T}$ in the transverse plane for a typical $W$ boson decay. The $u_{\|}$direction is defined to be along $\vec{p}_{T}^{\mu}$, the $u_{\perp}$ direction is perpendicular to the $\vec{p}_{T}^{\mu}$.

\subsection{Calorimeter-based Recoil Measurement}

Before we start modelling the recoil response and resolution, we need to calculate the hadronic recoil measured in the calorimeter first. The calorimeter-measured $\vec{u}_{T}^{\text {rec }}$ is 
the vector sum of energy deposited in all electromagnetic and hadronic calorimeter towers with $\eta$ coverage $|\eta|<3.6$ by assuming massless particles:

$$
\vec{u}_{T}^{\mathrm{rec}}=\left(\begin{array}{l}
u_{x} \\
u_{y}
\end{array}\right)=\left(\begin{array}{c}
\sum_{\text {all towers }} E \sin \theta \cos \phi \\
\sum_{\text {all towers }} E \sin \theta \sin \phi
\end{array}\right)
$$

Since muons also deposit some energy in the calorimeter system, to minimize the recoil energy measurement bias caused by the muon(s) from the $W / Z$ boson decay, we remove towers associated with the muon(s) from tower energy summation. Figure 10.2 shows the spatial distributions of mean $E_{T}$ per EM and hadronic calorimeter tower in the vicinity of the muon track using $W \rightarrow \mu \nu$ data events. Based on these distributions, we exclude the three towers indicated in the box.
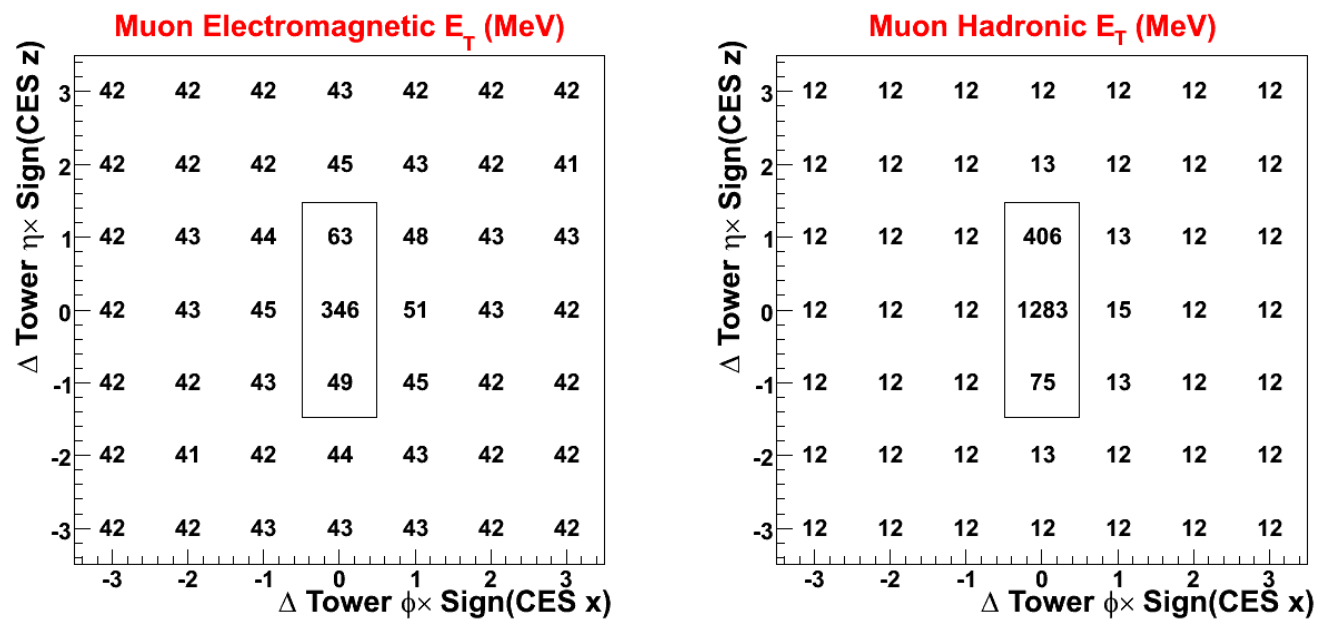

FiguRE 10.2: Grids for mean EM (left) and hadronic (right) energy in calorimeter towers surrounding the muon track using $W \rightarrow \mu \nu$ data events.

However, excluding the muon-associated towers also excludes the underlying energy deposited in those towers thus causing a bias in recoil calculation. Since this energy shows some dependence on $\eta, u_{\|}$and $u_{\perp}$, we parameterize its variation as 
functions of $\eta, u_{\|}$and $u_{\perp}$ from data using equivalent towers separated in $\phi$ and incorporate the parameterization into the fast simulation.

To facilitate recoil model tuning, we define axes in the transverse plane as illustrated in Figure 10.3. We define the $\eta$ axis as being parallel to the dilepton $\vec{p}_{T}(l l)$ axis and the $\xi$ axis perpendicular to the $\eta$ axis.

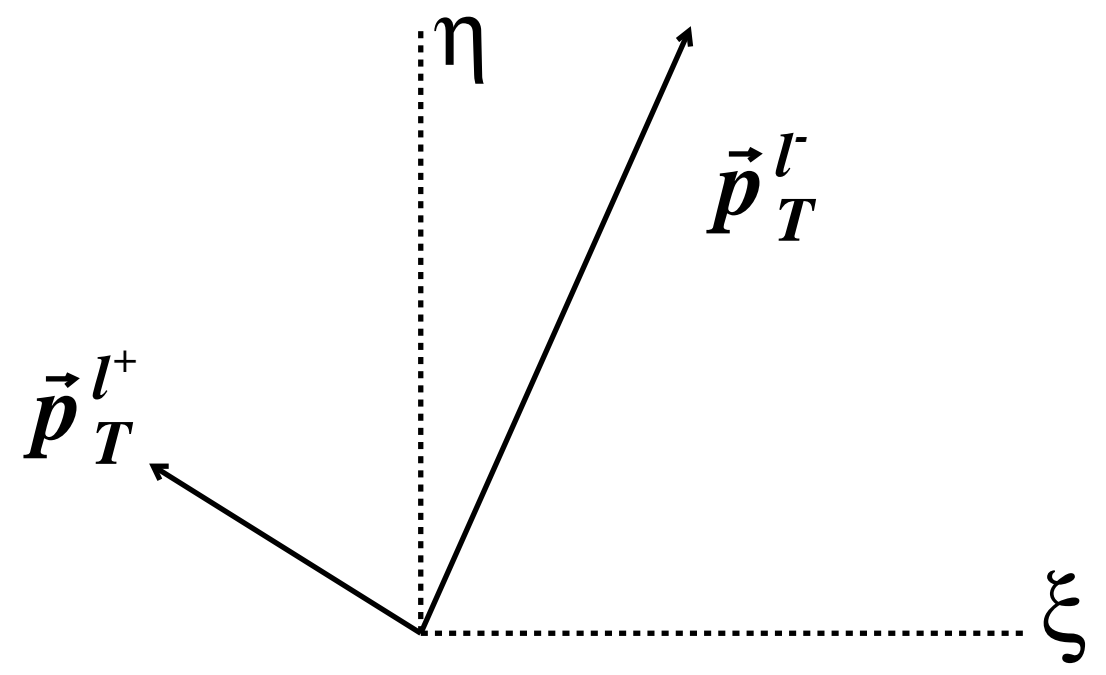

Figure 10.3: The $\vec{p}_{T}$ vectors of the two leptons in $Z \rightarrow l^{+} l^{-}$events. The $\eta$ axis is parallel to the $\vec{p}_{T}(l l)$ direction and the $\xi$ axis is perpendicular to it.

\subsection{Hadronic Recoil Response}

We parameterize the response and resolution of the calorimeter system to the recoil by using $Z \rightarrow \mu \mu$ and $Z \rightarrow e e$ events, where the 3 -momentum of the $Z$ boson can be measured precisely. The recoil model is first tuned with these $Z$ leptonic decays then applied to the $W$ boson events.

We model calorimeter's recoil response as the ratio of the true recoil magnitude $u_{T}^{\text {true }}$, which has the same value as $\left|p_{T}(Z \rightarrow l l)\right|$ but in opposite in direction with 
respect to $p_{T}(Z \rightarrow l l)$, and the calorimeter-measured value $u_{T}^{\text {rec }}$ :

$$
\begin{aligned}
R & \equiv \frac{\vec{u}_{T}^{\text {rec }} \cdot \hat{u}_{T}^{\text {true }}}{u_{T}^{\text {true }}} \\
& =a \frac{\ln (b+c \cdot r)}{\ln (b+15 c)}
\end{aligned}
$$

where $r=u_{T}^{\text {true }} / \mathrm{GeV} ; a, b$ and $c$ are constants determined from the fits to the $Z$ boson data. The functional form is chosen to decorrelate $a, b$ and $c$. To tune these parameters, we compare $p_{T}^{\eta}$-balance plot from data and simulation by minimizing the $\chi^{2}$. Figure 10.4 shows the dependence of mean $\eta$-direction $p_{T}$ balance on $p_{T}^{Z}$. The scale factor 0.65 applied to $p_{T}^{\eta}(Z)$ is chosen such that the mean value $\left\langle 0.65 p_{T}(l l)+u_{T}\right\rangle$ (the $y$ axis in Figure 10.4) is approximately zero. We find values of $a=0.645 \pm 0.002_{\text {stat }}$, $b=8.2 \pm 2.2_{\text {stat }}$ and $c=5.1 \pm 0.6_{\text {stat }}$. In the last $200 \mathrm{pb}^{-1}$ publication, term $c$ is fixed to 1 in the functional form of $R$.

To quantify the systematic uncertainties on $m_{W}$, we vary parameters $a, b$ and $c$ by $\pm 2 \sigma_{\text {stat }}$ to re-generate templates while keeping the pesudo-experiment histogram fixed. The difference in fitted $m_{W}$ central value between $+2 \sigma$ and $-2 \sigma$ variation is then converted into $1 \sigma$ by assuming the variation is linear. The signed $\delta m_{W} \mathrm{~s}$ are summarized in Table 10.1. The MC statistical errors $\left(\delta_{\mathrm{MC}}\right)$ of $m_{T}, p_{T}^{\mu}$ and $p_{T}^{\nu}$ mass fits are about $4.3 \mathrm{MeV}, 4.7 \mathrm{MeV}$ and $5.3 \mathrm{MeV}$, respectively. The conservative MC error on $\delta m_{W}$ can be obtained by assuming uncorrelated $\pm 2 \sigma$ variations:

$$
\Delta_{\delta_{\mathrm{MC}}} \approx \delta_{\mathrm{MC}} \cdot \sqrt{2} / 4 \approx 1.6 \mathrm{MeV}
$$

\subsection{Hadronic Recoil Resolution}

We use two components to model the recoil resolution: the jet resolution model for the high $p_{T}$ regime, and the underlying event model, which captures the spectator interactions and the multiple interactions, for the low $p_{T}$ regime. 


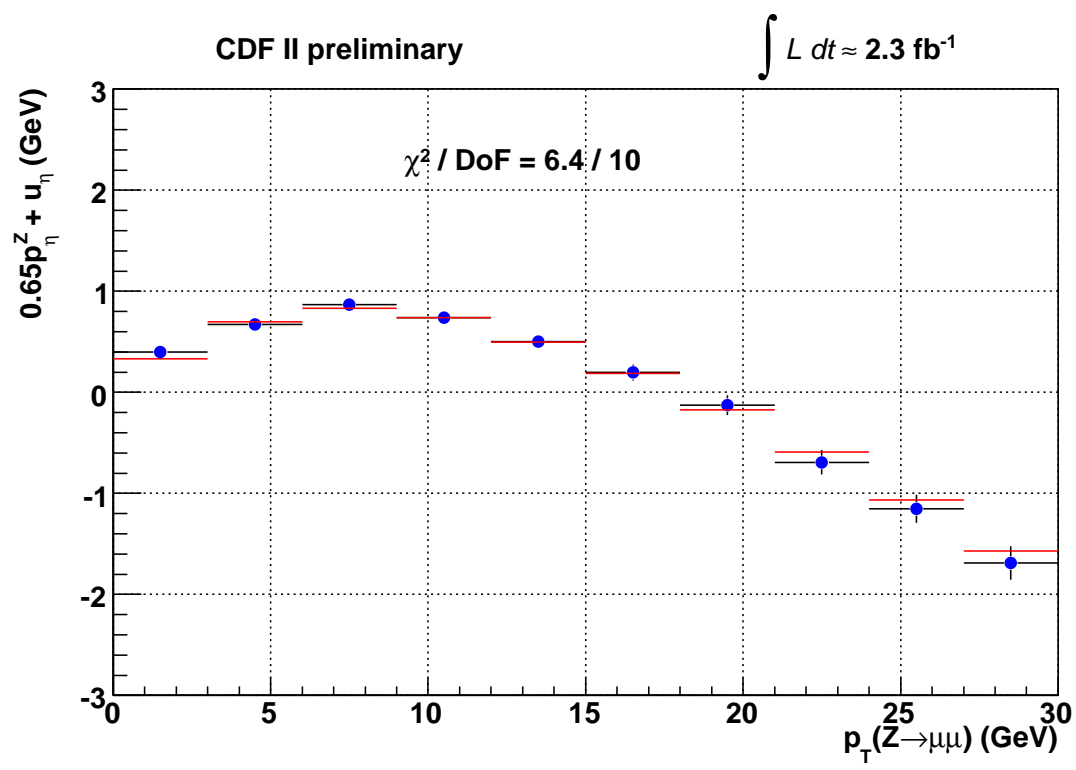

FigURE 10.4: Mean variation of $0.65 \cdot p_{T}^{\eta}(Z)+u_{T}^{\eta}$ as a function of $p_{T}^{Z \rightarrow l l}$ using $Z \rightarrow \mu \mu$ events. This plot, together with the corresponding plot using $Z \rightarrow e e$ events, is used in tuning recoil scale parameters. The red histograms are from DukeSim while the blue points are from data. The factor of 0.65 is chosen such that the mean value of $0.65 p_{T}^{\eta}(Z)+u_{T}^{\eta}$ is approximately 0 .

Table 10.1: Signed shifts in $m_{W}$ due to $1 \sigma$ variations in the recoil response parameters. The shifts are obtained by varying templates with pseudo-experiment histogram fixed. The Monte Carlo statistical uncertainty on the shifts are $\sim 1.6 \mathrm{MeV}$.

\begin{tabular}{ccccc}
\hline \hline $\begin{array}{c}\text { Input } \\
\text { parameter }\end{array}$ & $\begin{array}{c}\text { Central value } \\
\text { and statistical error }\end{array}$ & \multicolumn{3}{c}{ Shift $(\mathrm{MeV})$} \\
$m_{T}$ & $p_{T}^{\mu}$ & $p_{T}^{\nu}$ \\
\hline \hline$a$ & $0.645 \pm 0.002$ & +2 & +5 & -1 \\
$b$ & $8.2 \pm 2.2$ & +4 & -2 & +1 \\
$c$ & $5.1 \pm 0.6$ & +3 & +3 & -1 \\
\hline Response & & 5 & 6 & 2 \\
\hline \hline
\end{tabular}


The jet resolution model, which describes the hadronic activity, consists of the parameterization of jet energy fluctuation along the direction of $\vec{u}_{T}^{\text {true }}$ and the parameterization of the azimuthal angle fluctuation of $\vec{u}_{T}^{\text {rec }}$ relative to $\vec{u}_{T}^{\text {true }}$.

Similar to the $200 \mathrm{pb}^{-1} W$ mass analysis, the energy fluctuation along the direction of $\vec{u}_{T}^{\text {true }}$ is parameterized as a Gaussian with rms given by:

$$
\sigma_{\text {jet }}^{\text {energy }}=s_{\text {had }} \cdot \sqrt{u_{T}^{\text {true }}}
$$

where $s_{\text {had }}$ is the sampling term to be determined from data. This form captures the fact that the rms grows approximately as $\sqrt{u_{T}^{\text {true }}}$.

As one could imagine, the azimuthal angle $\phi$ of $\vec{u}_{T}^{\text {rec }}$ relative to the direction of $\vec{u}_{T}^{\text {true }}$ is dependent on the recoil transverse energy $u_{T}^{\text {true }}$. When $u_{T}^{\text {true }}$ gets higher, jets will get more collimated, leading to better angular resolution (smaller $\sigma \phi$ ). In the $200 \mathrm{pb}^{-1}$ analysis, we use a constant smearing $\sigma(\phi)$ to describe such an angle. In this $W$ mass analysis, we parameterize $\sigma(\phi)$ by a continuous 3-piece linear function in the ranges of $0<u_{T}^{\text {true }}<15 \mathrm{GeV}, 15 \mathrm{GeV}<u_{T}^{\text {true }}<30 \mathrm{GeV}$ and $u_{T}^{\text {true }}>30$ $\mathrm{GeV}$. Since we require $u_{T}^{\text {rec }}<15 \mathrm{GeV}$ for the mass measurement, the $u_{T}^{\text {true }}$ region above $30 \mathrm{GeV}$ is irrelevant for our concern and is set to be a constant. Reference points at $u_{T}^{\text {true }}=9.4 \mathrm{GeV}, 15 \mathrm{GeV}$ and $24.5 \mathrm{GeV}$ are chosen such that the fitted uncertainties on three parameters $(\alpha, \beta, \gamma)$ are uncorrelated. The functional form of $\sigma(\phi)$ is:

$$
\sigma\left(\phi\left(u_{T}^{\text {true }}\right)\right)= \begin{cases}\kappa_{1}\left(9.4-u_{T}^{\text {true }}\right)+\alpha & \text { if } u_{T}^{\text {true }}<15 \\ \beta & \text { if } u_{T}^{\text {true }}=15 ; \\ \kappa_{2}\left(24.5-u_{T}^{\text {true }}\right)+\gamma & \text { if } 15<u_{T}^{\text {true }}<30 .\end{cases}
$$

where $\kappa_{1,2}$ are coefficients fixed by continuity.

The soft component of hadronic resolution model consists of contributions from spectator interactions (SI) and multiple interactions (MI). 
The $\sum E_{T}$ distribution from SI $\left(\sum E_{T}(S I)\right)$ is obtained by using minimum-bias data events which are mostly inelastic $p \bar{p}$ collisions with small momentum transfer. There we have to account for the difference in the mean number of interactions at the same instantanous luminosity between $W / Z$ events and minimum-bias events. We deconvolute the $\sum E_{T}(M B)$ distribution obtained from minimum-bias data to the $\sum E_{T}$ distribution of a single interaction. A scale factor $N_{V}$ is then multiplied to $\sum E_{T}$ to accommodate the difference between the $\sum E_{T}$ from $Z$ event and a minimum-bias event.

The $\sum E_{T}$ distribution from $\mathrm{MI}\left(\sum E_{T}(M I)\right)$ is obtained by using zero-bias data collected at the same time as the $W / Z$ data. The zero-bias data has been sampled such that its instantaneous luminosity is identical to that of the $W / Z$ boson data. The obtained $\sum E_{T}(M I)$ histogram is then randomly sampled with the obtained value added to DukeSim.

The jet energy resolution parameter $s_{\text {had }}$ and the underlying event scaling factor $N_{V}$ are statistically uncorrelated by construction. The $r m s$ plot of $p_{T^{-}}$balance in $\eta$-projection $\left(\sigma\left(0.65 p_{T}^{\eta}(Z)+u_{T}^{\eta}\right)\right.$, see Figure 10.5) is used to tune $s_{\text {had }}$ and $N_{V}$. We find the following values give the best data-MC agreement:

$$
\begin{aligned}
s_{\text {had }} & =0.820 \pm 0.009_{\text {stat }} \\
N_{V} & =1.079 \pm 0.012_{\text {stat }}
\end{aligned}
$$

We use the rms of $p_{T}$-balance in $\xi$-projection $\left(\sigma\left(0.65 p_{T}^{\xi}(Z)+u_{T}^{\xi}\right)\right.$, see Figure 10.5) to tune jet angular resolution parameters $\alpha, \beta$ and $\gamma$. Their best-fit values are found to be

$$
\begin{aligned}
& \alpha=0.306 \pm 0.006_{\text {stat }} \\
& \beta=0.190 \pm 0.005_{\text {stat }} \\
& \gamma=0.144 \pm 0.004_{\text {stat }}
\end{aligned}
$$


Once we determine the recoil model parameters using $Z$ boson events, we can apply the fully tuned recoil model to the simulated $W$ boson sample. The uncertainties on $m_{W}$ fits from $s_{\text {had }}, N_{V}$ and angle smearing parameters $\alpha, \beta, \gamma$ are obtained in the same way as the uncertainties from recoil response parameters. Templates are generated when varying parameters by $\pm 2 \sigma$ while pseudo-experiment histogram unchanged. Use the same method introduced before, we convert the change in fitted $m_{W}$ to $1 \sigma$ by assuming linear variation of fitted $m_{W}$ with respect to the varying parameter. Table 10.2 summarizes the shifts on $m_{W}$ due to the $1 \sigma$ variation of the recoil parameters. The MC statistical errors on $m_{W}$ shifts are estimated to be 1.6 $\mathrm{MeV}$ as before. Combining with the systematic uncertainties arising from response parameters, we get $9 \mathrm{MeV}, 7 \mathrm{MeV}, 14 \mathrm{MeV}$ for $m_{T}, p_{T}^{\mu}$ and $p_{T}^{\nu}$, respectively.

Table 10.2: Signed shifts in $m_{W}$ due to $1 \sigma$ variations in the recoil resolution parameters. The shifts are obtained by varying templates with pseudo-experiment histogram fixed. The Monte Carlo statistical uncertainty on the shifts are $\sim 1.6 \mathrm{MeV}$.

\begin{tabular}{ccccc}
\hline \hline Input & Central value & \multicolumn{3}{c}{ Shift $(\mathrm{MeV})$} \\
parameter & and statistical error & $m_{T}$ & $p_{T}^{\mu}$ & $p_{T}^{\nu}$ \\
\hline \hline$s_{\text {had }}$ & $0.820 \pm 0.009$ & +1 & +2 & 0 \\
$N_{V}$ & $1.079 \pm 0.012$ & +5 & -2 & -13 \\
$\alpha$ & $0.306 \pm 0.006$ & -4 & 0 & -6 \\
$\beta$ & $0.190 \pm 0.005$ & 0 & +3 & -1 \\
$\gamma$ & $0.144 \pm 0.004$ & -2 & -3 & +1 \\
\hline Resolution & & 7 & 5 & 14 \\
\hline \hline
\end{tabular}

\subsection{Recoil Model Cross-Checks}

Three important quantities are chosen for the comparison of data and MC-predicted distributions from $W$ boson: the projections of the recoil along $\left(u_{\|}\right)$and perpendicular to $\left(u_{\perp}\right)$ the charged lepton direction, and the amplitude of transverse recoil $u_{T}$. 

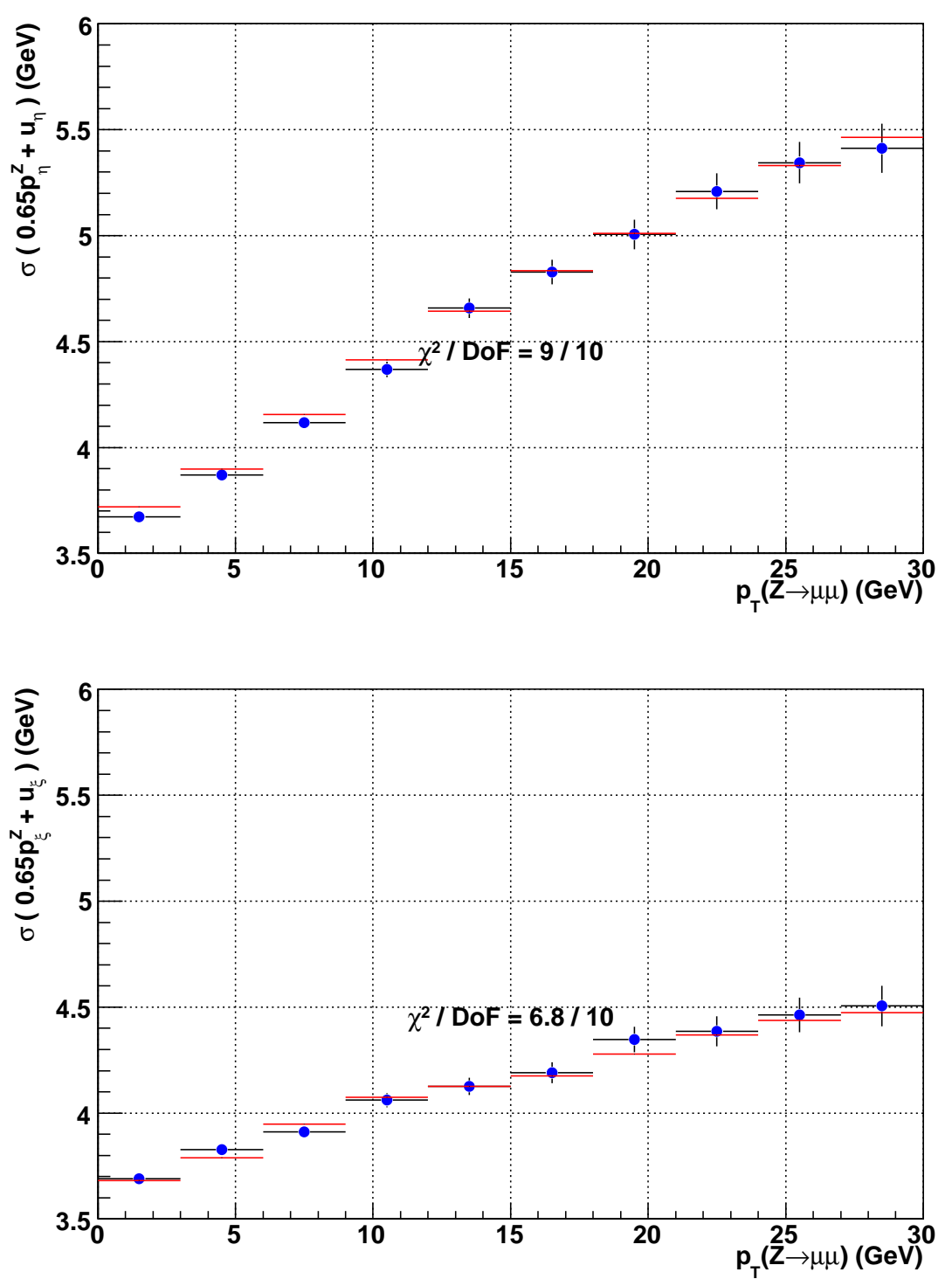

FIGURE 10.5: RMS variation of $0.65 \cdot p_{T}^{\eta / \xi}(Z)+u_{T}^{\eta / \xi}$ as a function of $p_{T}^{Z \rightarrow l l}$ using $Z \rightarrow \mu \mu$ events. The top plot, together with the corresponding plot using $Z \rightarrow e e$ events, is used to tune jet energy resolution parameter $s_{\text {had }}$ and the underlying event scaling parameter $N_{V}$. The bottom plot, together with the corresponding plot using $Z \rightarrow e e$ events, is used to tune the jet angular resolution parameters $\alpha, \beta$ and $\gamma$. The data are shown in blue points and the simulation is shown in red histogram. 
Several factors affect the $u_{\|}$distribution: lepton efficiency measurement as a function of $u_{\|}$, lepton hole correction, $W$ boson $p_{T}$ and its decay angular distributions. The recoil response and resolution model also contributes to $u_{\|}$distribution. The data-MC agreement as illustrated in Figure 10.6 shows our simulation describes the $W$ boson data well.

The $u_{\perp}$ distribution is mostly sensitive to the recoil resolution. Figure 10.7 compares the $u_{\perp}$ distributions from data and from MC. No evident disagreement is observed.

As shown in Figure 10.8, the recoil energy $u_{T}$ is also modelled well by the simulation. The mean of $u_{T}$ spectrum is mainly controlled by the recoil response $R$ and the boson $p_{T}$, while the spread of $u_{T}$ spectrum is mostly affected by the resolution.

Figure 10.9 shows data-MC comparison of the angular distribution of $\vec{u}_{T}$ relative to the muon direction. Good agreement is also observed.

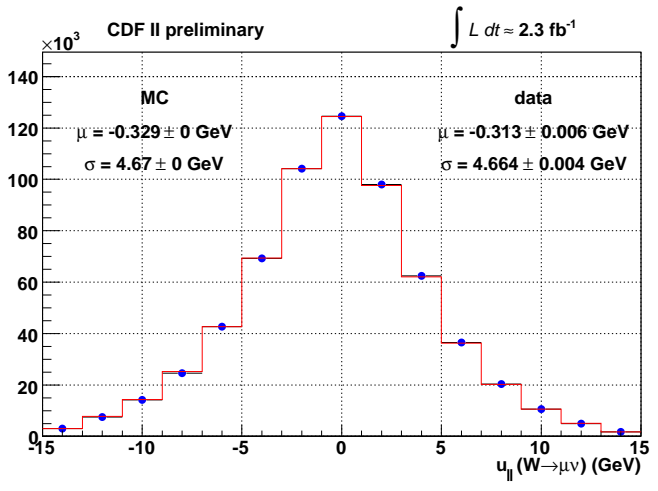

Figure 10.6: Comparison of $u_{\|}$between data (blue points) and simulation (red histogram) using $W \rightarrow \mu \nu$ events. The quoted uncertainties are statistical only. 


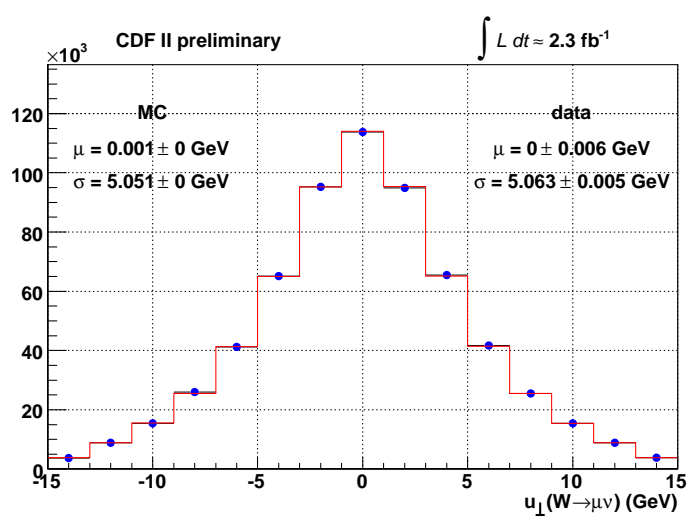

FiguRE 10.7: Comparison of $u_{\perp}$ distribution between data (blue points) and simulation (red histogram) using $W \rightarrow \mu \nu$ events. The quoted uncertainties are statistical only.

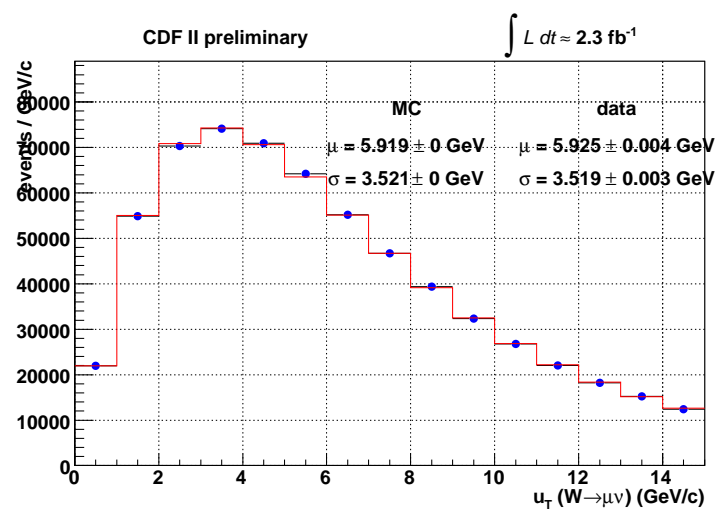

Figure 10.8: Comparison of $u_{T}$ distribution between data (blue points) and simulation (red histogram) using $W \rightarrow \mu \nu$ events. 


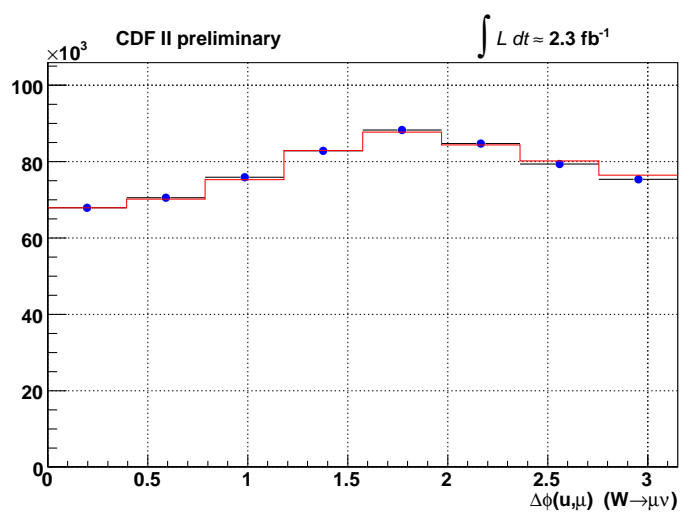

Figure 10.9: Comparison of $\Delta \phi_{u, \mu}$ between data (blue points) and simulation (red histogram) using $W \rightarrow \mu \nu$ events. 
As mentioned in Chapter 4, the $W$ mass is extracted in this measurement by performing a binned maximum likelihood fit to three kinematic distributions: transverse mass $\left(m_{T}\right)$, charged lepton transverse momentum $\left(p_{T}^{\mu}\right)$ and neutrino transverse momentum $\left(p_{T}^{\nu}\right)$. Background lineshapes from different background sources are normalized first according to their estimated fractions. They are then added to the simulated kinematic distribution to form a combination of simulated signal and background. This summed simulation lineshape is then normalized to the data lineshape by requiring the total number of simulated events in the specified fit region to be equal to the total number of events in the data distribution in the same region. Since this normalized simulation template gives the expected number of events in each bin, we can use it to calculate the Poisson probability of each data bin and form the total likelihood by multiplying the Poisson probabilities over all the bins in the chosen fitting window. In this way, we can calculate a total likelihood for each simulated template. As the input $m_{W}$ value varies, the simulated template and the corresponding likelihood will also vary. The input $m_{W}$ value which maximizes the caclulated likelihood gives the best match to the data distribution and thus gives the most probable $W$ boson 
mass. The results shown in this chapter is unblinded with the approval of the CDF Collaboration. The random offset which was first drawn from a uniform distribution within the range $[-75,75] \mathrm{MeV}$ then added to the real fitted value is already removed from the blinded fit.

In this chapter, we summarize the fitting results to $m_{T}, p_{T}^{\mu}$ and $p_{T}^{\nu}$ distributions together with the systematic uncertainties. We also study the correlations among the three fitting variables $m_{T}, p_{T}^{\mu}$ and $p_{T}^{\nu}$.

\subsection{Fitted $m_{W}$ Central Value and Statistical Error}

We generate $W$ boson events with RESBos at mass values of $80 \mathrm{GeV}$ and $81 \mathrm{GeV}$. Though RESBOs package captures the relevant QCD physics and models the $W p_{T}$ distribution well, it does not describe QED physics involved in $W$ boson production and decay. To account for the relevant QED process such as final-state photon radiation, we update decay information of the RESBOS $W$ boson events according to the QED description given by PHotos. We then feed RESBOs and PHOTOs convoluted $W$ boson events into our fast detector simulation (DukeSim). A series of templates, which can be used to fit against the data, are produced by using a linear extrapolation between the two simulated templates generated at $80 \mathrm{GeV}$ and $81 \mathrm{GeV}$ via reweighting according to the relativistic Breit-Wigner distribution

$$
\frac{d \sigma}{d m} \propto \frac{m^{2}}{\left(m^{2}-M_{W}^{2}\right)^{2}+m^{4} \Gamma_{W}^{2} / M_{W}^{2}}
$$

where $m$ is the invariant mass of the propagator. We use the $W$ boson width $\Gamma_{W}=$ $2.094 \mathrm{GeV}$ to generate the fitting templates. Figure 11.1 shows the transverse mass $m_{T}$ templates used to extract $m_{W}$. 


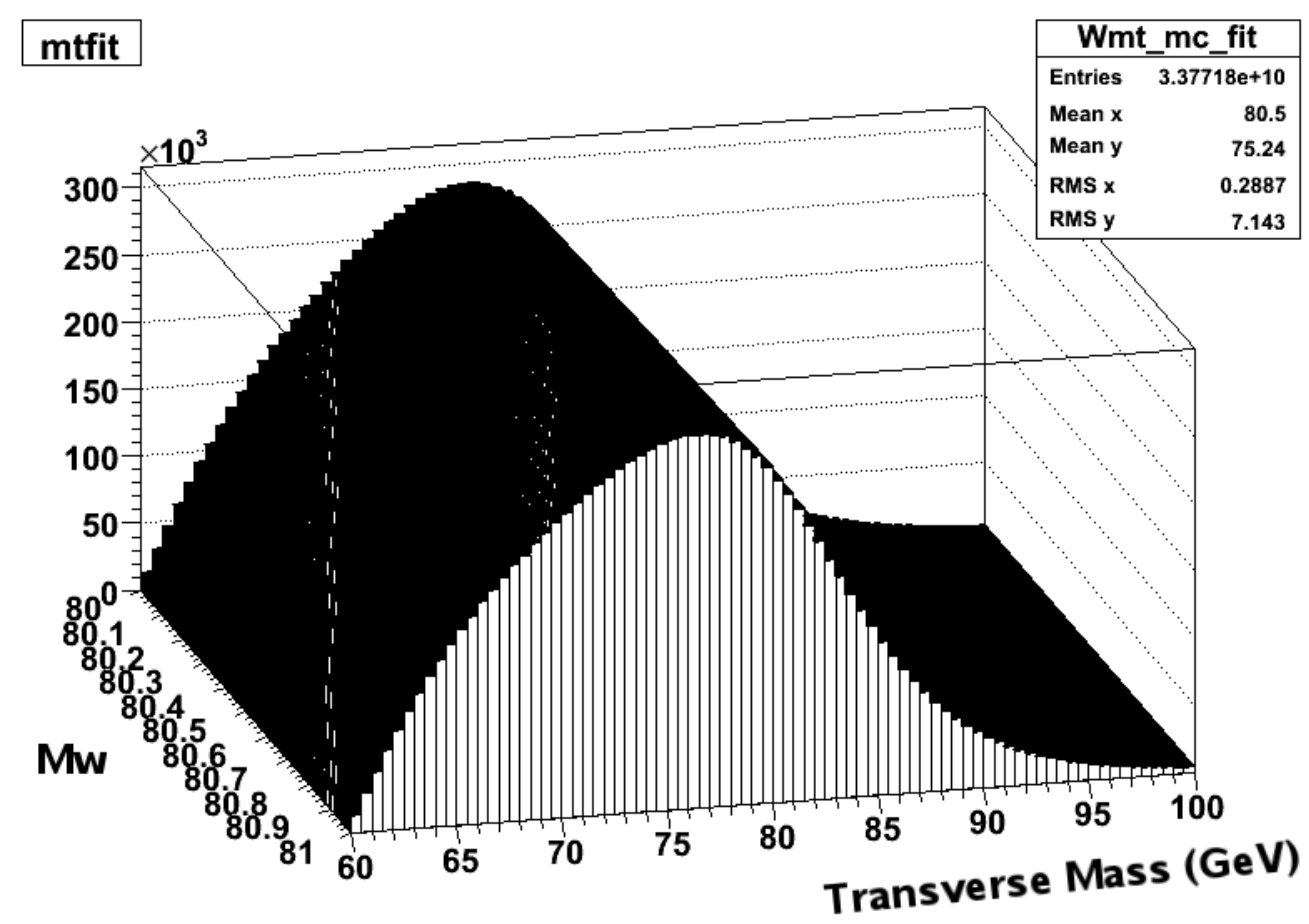

Figure 11.1: Transverse mass templates used to extract the $W$ boson mass $m_{W}$.

\subsection{Transverse Mass $m_{T}$ Fit Result}

The $m_{T}$ distribution fit result is shown in Figure 11.2. Normalized background shapes are also shown in the same plot. The $m_{W}$ is fitted in the range of $65 \mathrm{GeV}<m_{T}<90$ $\mathrm{GeV}$ and is found to be $m_{W}=80379 \pm 16_{\text {stat }} \mathrm{MeV}$, where the error term is statistical only. The systematic uncertainties on $m_{W}$ using $m_{T}$ distribution is given in Table 11.1. Combining statistical and systematic uncertainties, we obtain $m_{W}$ from $m_{T}$ fits to be

$$
\begin{aligned}
m_{W}\left(m_{T}\right) & =80379 \pm 16_{\text {stat }} \pm 16_{\text {syst }} \mathrm{MeV} \\
& =80379 \pm 23 \mathrm{MeV}
\end{aligned}
$$




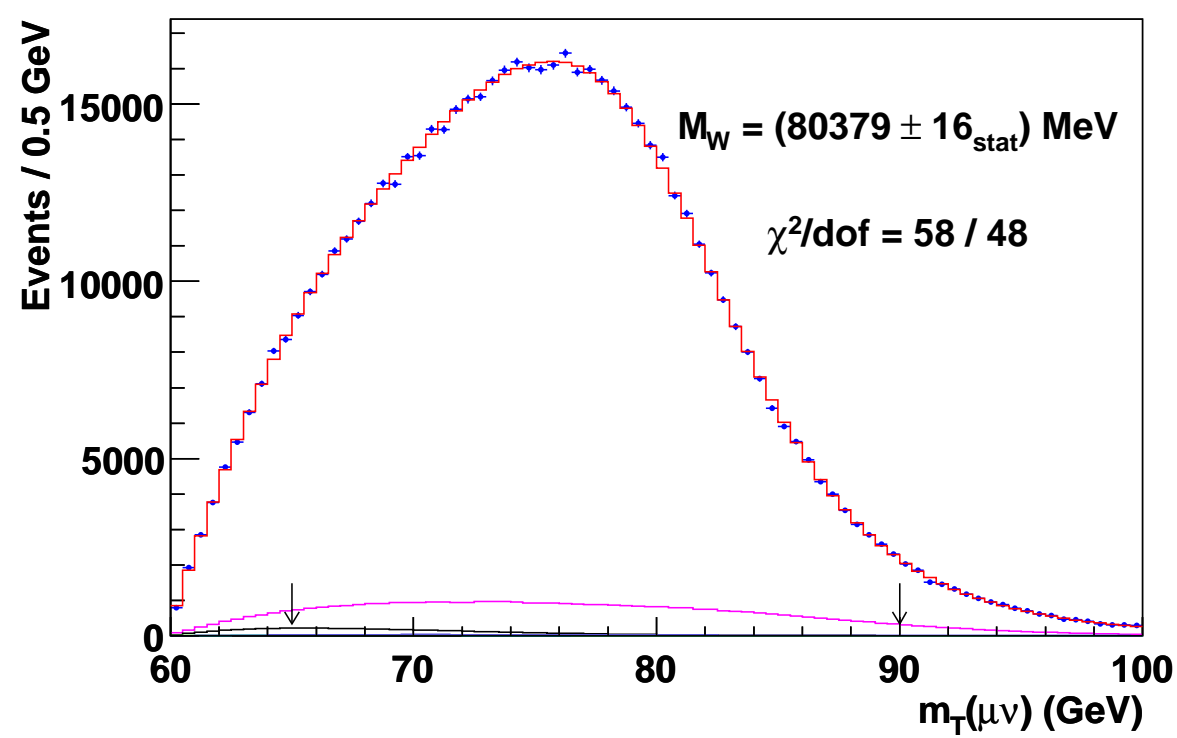

Figure 11.2: The $m_{T}$ distribution for $W$ boson decays to $\mu \nu$ with data in blue points and simulation in solid line.

Table 11.1: Systematic and statistical uncertainties on measured $m_{W}$ using $m_{T}$ fit.

\begin{tabular}{lc}
\hline \hline Uncertainties on $m_{T}$ fit for $m_{W}$ in the $W \rightarrow \mu \nu$ sample \\
\hline Source & Uncertainty $(\mathrm{MeV})$ \\
\hline Lepton Momentum Scale & 7 \\
Lepton Energy Resolution & 1 \\
Recoil Energy Scale & 5 \\
Recoil Energy Resolution & 7 \\
$u$ efficiency & 0 \\
Lepton Tower Removal & 2 \\
Backgrounds & 3 \\
$p_{T}(W)$ model $\left(g_{2}, g_{3}, \alpha_{s}\right)$ & 3 \\
Parton Distributions & 10 \\
QED Radiation & 4 \\
\hline Total Systematics & 16 \\
\hline Statistical & 16 \\
\hline Total & 23 \\
\hline \hline
\end{tabular}




\subsection{Charged Lepton $p_{T}^{\mu}$ Fit Result}

Figure 11.3 shows the $p_{T}^{\mu}$ fit result in the range of $32 \mathrm{GeV}<p_{T}^{\mu}<48 \mathrm{GeV}$. Simliar to Figure 11.3, background contributions in data are shown in the $p_{T}^{\mu}$ spectrum. The statistical uncertainty and systematic uncertainties on $m_{W}$ are summarized in Table 11.2. The $m_{W}$ from $p_{T}^{\mu}$ fit is measured to be

$$
\begin{aligned}
m_{W}\left(p_{T}^{\mu}\right) & =80348 \pm 18_{\text {stat }} \pm 18_{\text {syst }} \mathrm{MeV} \\
& =80348 \pm 25 \mathrm{MeV}
\end{aligned}
$$

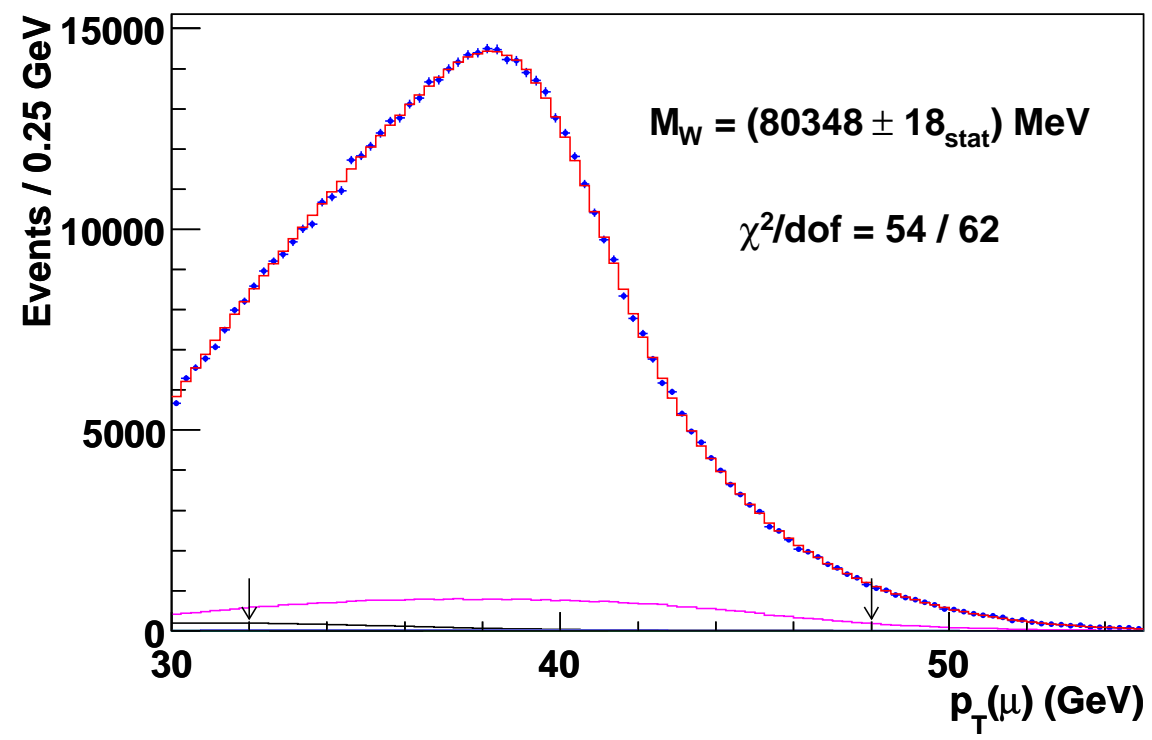

Figure 11.3: The $p_{T}^{\mu}$ distribution for $W$ boson decays to $\mu \nu$ with data in blue points and simulation in solid line.

\subsection{Missing Transverse Momentum $p_{T}^{\nu}$ Fit Result}

Figure 11.4 shows the $p_{T}^{\nu}$ fit result in the range of $32 \mathrm{GeV}<p_{T}^{\nu}<48 \mathrm{GeV}$. The statistical uncertainty and systematic uncertainties on $p_{T}^{\nu}$ are listed in Table 11.3. 
Table 11.2: Systematic and statistical uncertainties on measured $m_{W}$ using $p_{T}^{\mu}$ fit.

\begin{tabular}{lc}
\hline \hline Uncertainties on $p_{T}^{\mu}$ fit for $m_{W}$ in the $W \rightarrow \mu \nu$ sample \\
\hline Source & Uncertainty $(\mathrm{MeV})$ \\
\hline Lepton Momentum Scale & 7 \\
Lepton Energy Resolution & 1 \\
Recoil Energy Scale & 6 \\
Recoil Energy Resolution & 5 \\
$u$ efficiency & 1 \\
Lepton Tower Removal & 0 \\
Backgrounds & 5 \\
$p_{T}(W)$ model $\left(g_{2}, g_{3}, \alpha_{s}\right)$ & 9 \\
Parton Distributions & 9 \\
QED Radiation & 4 \\
\hline Total Systematics & 18 \\
\hline Statistical & 18 \\
\hline Total & 25 \\
\hline \hline
\end{tabular}

The $m_{W}$ from $p_{T}^{\nu}$ fit is found to be

$$
\begin{aligned}
m_{W}\left(p_{T}^{\nu}\right) & =80406 \pm 22_{\text {stat }} \pm 20_{\text {syst }} \mathrm{MeV} \\
& =80406 \pm 30 \mathrm{MeV} .
\end{aligned}
$$

\subsection{Cross-Checks}

The $W$ boson candidate sample is splitted in four ways into sub-samples to perform cross-checks. Firstly, we perform individual mass fits for $W^{+}$and $W^{-}$events. Secondly, we split the $W$ events based on the geometric locations of the decay muon within the COT into two samples with $\phi_{\mu}>0$ and $\phi_{\mu}<0$, which corresponds to the upper half and lower half of the COT, respectively. We also compared $m_{W}$ results by using muons in the left half $\left(\phi_{\mu} \in(-\pi,-\pi / 2) \cup(\pi / 2, \pi)\right)$ and the right half $\left(\phi_{\mu} \in(-\pi / 2, \pi / 2)\right)$ of COT. Lastly, we split the $W$ sample into two different run ranges with roughly the same statistics. No $m_{W}$ difference is found in the first three 


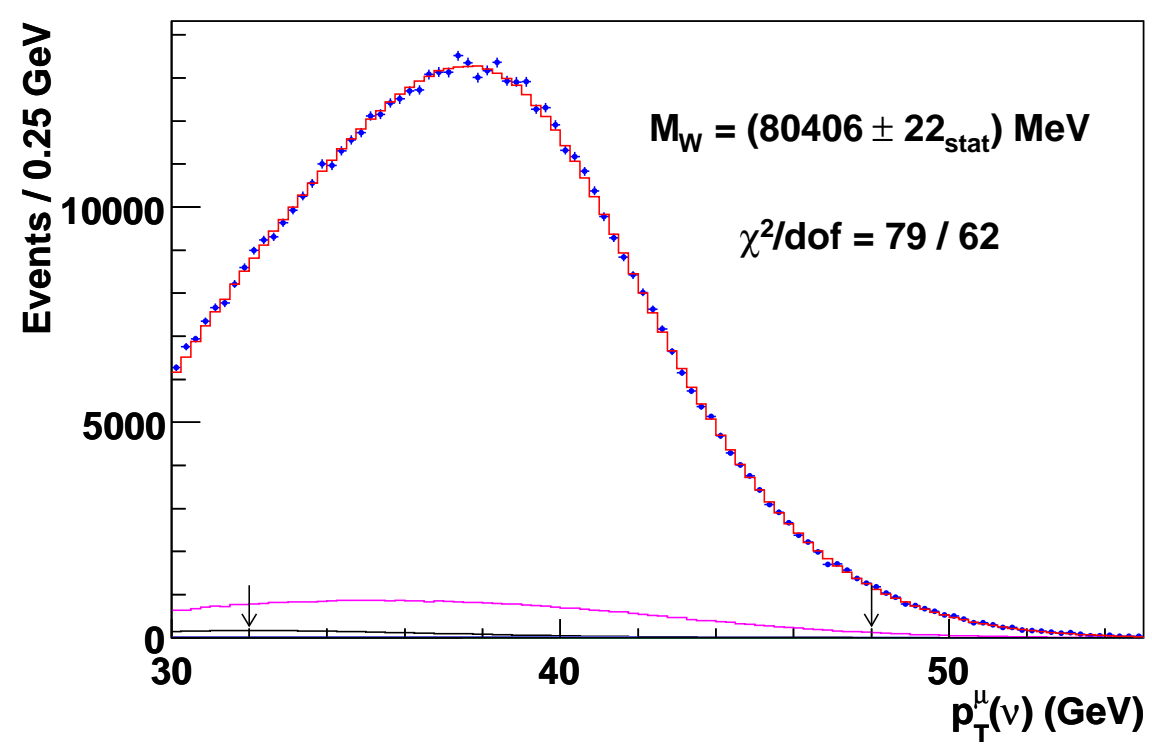

Figure 11.4: The $\mathbb{E}_{T}$ distribution for $W$ boson decays to $\mu \nu$ with data in blue points and simulation in solid line.

Table 11.3: Systematic and statistical uncertainties on measured $m_{W}$ using $p_{T}^{\nu}$ fit.

\begin{tabular}{lc}
\hline \hline Uncertainties on $p_{T}^{\nu}$ fit for $m_{W}$ in the $W \rightarrow \mu \nu$ sample \\
\hline Source & Uncertainty $(\mathrm{MeV})$ \\
\hline Lepton Momentum Scale & 7 \\
Lepton Energy Resolution & 1 \\
Recoil Energy Scale & 2 \\
Recoil Energy Resolution & 11 \\
$u$ efficiency & 2 \\
Lepton Tower Removal & 4 \\
Backgrounds & 6 \\
$p_{T}(W)$ model $\left(g_{2}, g_{3}, \alpha_{s}\right)$ & 4 \\
Parton Distributions & 11 \\
QED Radiation & 4 \\
\hline Total Systematics & 20 \\
\hline Statistical & 22 \\
\hline Total & 30 \\
\hline \hline
\end{tabular}


Table 11.4: Cross-checks of fitted $m_{W}$ via $m_{T}$ fit using sub-samples. $\phi_{\mu}>0$ and $\phi_{\mu}<0$ correspond to the upper half and the lower half of the COT, respectively. $\phi_{\mu}^{\text {left }}$ and $\phi_{\mu}^{\text {right }}$ corresponds to the left half $\left(\phi_{\mu} \in(-\pi,-\pi / 2) \cup(\pi / 2, \pi)\right)$ and the right half $\left(\phi_{\mu} \in(-\pi / 2, \pi / 2)\right)$ of the COT. The early and the late data is for runs with run number $<$ and $>222000$, respectively. The $59 \mathrm{MeV}$ systematic uncertainty in $m_{W}^{\mu^{+}}-m_{W}^{\mu^{-}}$is from the statistical uncertainty of COT alignment parameters $a$ and $c$ (see reference [87]). The $\sim 3 \sigma$ difference between early and late runs suggests the best-tuned recoil parameters using all runs does not describe data in early/late run ranges, thus are run-dependent. To get better agreement in $m_{W}$, the recoil parameters need to be retuned for different run ranges.

\begin{tabular}{lc}
\hline \hline Cross-check & Fit difference in $m_{W}(\mathrm{MeV})$ \\
\hline$m_{W}\left(\mu^{+}\right)-m_{W}\left(\mu^{-}\right)$ & $42 \pm 35 \pm 59_{\mathrm{COT}}$ \\
$m_{W}\left(\phi_{\mu}>0\right)-m_{W}\left(\phi_{\mu}<0\right)$ & $13 \pm 35$ \\
$m_{W}\left(\phi_{\mu}^{\text {left }}\right)-m_{W}\left(\phi_{\mu}^{\text {right }}\right)$ & $37 \pm 35$ \\
$m_{W}($ early $)-m_{W}($ late $)$ & $120 \pm 38$ \\
\hline \hline
\end{tabular}

cross-checks. There is a $\sim 3 \sigma$ difference in the fourth check, indicating the global recoil parameters do not describe the recoils in sub-samples and need retuning. All check results are summarized in Table 11.4. Cross-checks using $p_{T}^{\mu}$ fit, which is a little less sensitive to the recoil model as compared to $m_{T}$ or $p_{T}^{\nu}$, also shows similar results [93].

Figure 11.5 shows the variations of the fitted $W$ boson mass values, relative to nominal results, as the fit windows are varied. The left three plots are for changing the lower edges of $m_{T}, p_{T}^{\mu}$ and $p_{T}^{\nu}$ distributions while the upper edges fixed at 90 $\mathrm{GeV}, 48 \mathrm{GeV}$ and $48 \mathrm{GeV}$, respectively. The right three plots correspond to the variations of the upper edges of $m_{T}, p_{T}^{\mu}$ and $p_{T}^{\nu}$ distributions while the lower edges fixed at $65 \mathrm{GeV}, 32 \mathrm{GeV}$ and $32 \mathrm{GeV}$, respectively. The error bars are the expected $1 \sigma$ statistical variations from simulation pseudoexperiments (see Appendix I for detailed discussion). From Figure 11.5 we can see the shifts in fitted $m_{W}$ are within the corresponding $m_{W}$ statistical uncertainties and no apparent dependence on the choice of fit window is observed. 


\subsection{Correlations between Mass Fits}

The correlation coefficients between the different $W$ mass fits are needed for two reasons. Firstly, we need to check the consistency of the three data fit values from $m_{T}, p_{T}^{\mu}$ and $p_{T}^{\nu}$. Secondly, the three data fit values need to be combined into a single value to be quoted as the final $m_{W}$ measurement using the $W \rightarrow \mu \nu$ channel.

We obtain the statistical correlations between $m_{T}, p_{T}^{\mu}$ and $p_{T}^{\nu}$ fits by using large Monte Carlo samples. We use PYTHIA [76] Version 6.208 to generate $W \rightarrow \mu \nu$ events to provide the same lepton-momentum 4-vector files that are obtained from RESBOS for the data fits. These vector files are later processed by the same DukeSim detector simulation that is used for fitting the data. A vector file containing 100 million $W \rightarrow \mu \nu$ PYTHIA events are generated at each of the three $W$ mass input values at $80 \mathrm{GeV}, 80.45 \mathrm{GeV}$ and $81 \mathrm{GeV}$. The two vector files generated at $m_{W}=80 \mathrm{GeV}$ and $m_{W}=81 \mathrm{GeV}$ are used to construct fitting templates by reweighting the events from $m_{W}=80 \mathrm{GeV}$ and $m_{W}=81 \mathrm{GeV}$ according to the Breit-Wigner distribution, while the vector file generated at $m_{W}=80.45 \mathrm{GeV}$ is splitted into 400 sub-samples with equal statistics to form pseudo-data files.

Each of the 400 psedo-data files are then fitted against the templates. The 400 groups of $m_{T}, p_{T}^{\mu}$ and $p_{T}^{\nu}$ fitting results are used to estimate the population correlation coefficient $\rho$ by constructing the sample correlation coefficient $r$ :

$$
\begin{aligned}
r_{X Y} & =\frac{E(X Y)-E(X) E(Y)}{s_{X} s_{Y}} \\
& =\frac{\frac{1}{n} \sum_{i=1}^{n} x_{i} y_{i}-\bar{x} \bar{y}}{\sqrt{\frac{1}{n-1}\left(\sum_{i=1}^{n} x_{i}^{2}-n \bar{x}^{2}\right)} \sqrt{\frac{1}{n-1}\left(\sum_{i=1}^{n} y_{i}^{2}-n \bar{y}^{2}\right)}},
\end{aligned}
$$

where $x_{i}\left(y_{i}\right)$ is the $i^{t h}$ value of fit type $\mathrm{X}(\mathrm{Y}), S_{X}\left(S_{Y}\right)$ is the sample variance of fit type $\mathrm{X}(\mathrm{Y}), \bar{x}=\frac{1}{n} \sum_{i=1}^{n} x_{i}$ and $\bar{y}=\frac{1}{n} \sum_{i=1}^{n} y_{i}, n$ is the number of $\left(x_{i}, y_{i}\right)$ pairs. The $1 \sigma$ error on $\rho_{X Y}$ can be estimated using Fisher's $z$ transformation on the sample 
correlation coefficient $r_{X Y}$. The Fisher's $z$ transformation is given by [88]:

$$
z=\frac{1}{2} \ln \left(\frac{1+r}{1-r}\right)
$$

where $z$ is approximately normally distributed with mean $\frac{1}{2} \ln \left(\frac{1+\rho}{1-\rho}\right)$ and standard error $\frac{1}{\sqrt{n-3}}$. Thus we have

$$
\begin{aligned}
& z^{+\sigma_{z}} \approx \frac{1}{2} \ln \left(\frac{1+r}{1-r}\right)+\frac{1}{\sqrt{n-3}} \\
& z^{-\sigma_{z}} \approx \frac{1}{2} \ln \left(\frac{1+r}{1-r}\right)-\frac{1}{\sqrt{n-3}}
\end{aligned}
$$

which lead to

$$
\begin{aligned}
& r^{+\sigma_{r}}=r+\sigma_{r} \approx \frac{e^{2\left(z^{+\sigma z}\right)}-1}{e^{2\left(z^{+\sigma z}\right)}+1} \\
& r^{-\sigma_{r}}=r-\sigma_{r} \approx \frac{e^{2\left(z^{-\sigma z}\right)}-1}{e^{2\left(z^{-\sigma_{z}}\right)}+1} .
\end{aligned}
$$

We symmetrize the $+1 \sigma$ and $-1 \sigma$ to get

$$
\sigma_{\rho} \approx \sigma_{r} \approx \frac{r^{+\sigma_{r}}-r^{-\sigma_{r}}}{2}
$$

Figure 11.6 shows the statistical correlations among $m_{T}, p_{T}^{\mu}$ and $p_{T}^{\nu}$ fits. The correlation coefficients are summarized in Table 11.5. The same procedure can be applied to obtain the statistical correlation coefficients in $W \rightarrow e \nu$ channel [89]. See Appendix J for detailed discussions.

In this $m_{W}$ analysis, we do not consider the systematic uncertainties on the correlation coefficients for two reasons. Firstly, the systematic effects on the physics parameters are pretty small (on the order of $1 \%$ of themselves), which would affect the statistical correlation coefficients on the order of $1 \%$. In comparison, the MC 
Table 11.5: Statistical correlations between $m_{T}, p_{T}^{\mu}$ and $p_{T}^{\nu}$ fits in $W \rightarrow \mu \nu$ channel. The statistical errors are obtained by using Fisher's z-transformation.

\begin{tabular}{lc}
\hline \hline$W \rightarrow \mu \nu$ fit variable & Correlation Coefficient \\
\hline$m_{T}$ vs. $p_{T}^{\mu}$ & $0.672 \pm 0.028$ \\
$m_{T}$ vs. $p_{T}^{\nu}$ & $0.658 \pm 0.028$ \\
$p_{T}^{\mu}$ vs. $p_{T}^{\nu}$ & $0.255 \pm 0.047$ \\
\hline \hline
\end{tabular}

statistical errors on the correlation coefficients are $3 \% \sim 4 \%$, already at least as big or bigger than the potential systematic effect. Secondly, higher precision of the correlation coefficients are not needed as the purposes are to check the consistency of all three mass fits and to combine them into a single value.

\subsubsection{Monte Carlo Study of Statistical Correlation Coefficient Errors}

We use Monte Carlo simulation to cross-check the errors on the statistical correlation coefficients calculated from Fisher's $z$ transformation. For ease of explanation, we use random variables $x_{1}, x_{2}, x_{3}$ to represent $m_{T}, p_{T}^{\mu}$ and $p_{T}^{\nu}$ fits which we want to generate from simulation. The $1 \sigma$ statistical errors on $x_{1}, x_{2}$ and $x_{3}$ are set to be the same as those obtained from data fits, i.e., $\sigma_{1}=0.015 \mathrm{GeV}, \sigma_{2}=0.017 \mathrm{GeV}$, and $\sigma_{3}=0.021 \mathrm{GeV}$. The correlation coefficients among $x_{1}, x_{2}$ and $x_{3}$ are set to be the numbers shown in Table 11.5 with $r_{1,2}=r_{2,1}=0.672, r_{1,3}=r_{3,1}=0.658$ and $r_{2,3}=r_{3,2}=0.255$. From the above information, the covariance matrix $\boldsymbol{\Sigma}$ can be constructed as

$$
\boldsymbol{\Sigma}=\left(\begin{array}{ccc}
\sigma_{1}^{2} & r_{1,2} \sigma_{1} \sigma_{2} & r_{1,3} \sigma_{1} \sigma_{3} \\
r_{2,1} \sigma_{1} \sigma_{2} & \sigma_{2}^{2} & r_{2,3} \sigma_{2} \sigma_{3} \\
r_{3,1} \sigma_{1} \sigma_{3} & r_{3,2} \sigma_{2} \sigma_{3} & \sigma_{3}^{2}
\end{array}\right)
$$

Correlated random variables $x_{i}(i=1,2,3)$ can be generated from independent random variables $z_{i}(i=1,2,3)$ using a matrix decomposition method called Singular 
Value Decomposition [90] (SVD) according to the formula:

$$
\mathbf{X}=\mathbf{M}+\left(\mathbf{U D}^{\mathbf{1} / \mathbf{2}}\right) \mathbf{Z}
$$

where $\mathbf{X}$ is a vector of random variables $x_{i}(i=1, \cdots, n), \mathbf{M}$ is a vector of $m_{i}$ $(i=1, \cdots, n)$ with $m_{i}$ equal to $\mathrm{E}\left(x_{i}\right)$, the mean value of $x_{i} . \quad \mathbf{Z}$ is a vector of $n$ independent $\mathrm{N}(0,1)$ random variables; $\mathbf{U}$ is the matrix of $n$ eigenvectors obtained from covariance matrix $\boldsymbol{\Sigma}$, with the $i$-th column of $\mathbf{U}$ be the eigenvector correponding to the $i$-th eigenvalue $\lambda_{i}$ from $\Sigma$; $\mathbf{D}$ is a $n \times n$ diagonal matrix with diagonal elements to be the eigenvalues $\left(\lambda_{1}, \lambda_{2}, \cdots, \lambda_{n}\right)$ of covariance matrix $\boldsymbol{\Sigma} . \mathbf{D}^{\mathbf{1} / \mathbf{2}}$ is a diagonal matrix with diagonal elements $\sqrt{\lambda_{1}}, \sqrt{\lambda_{2}}, \cdots, \sqrt{\lambda_{n}}$.

In our study with $n=3$, we assume the expected value from all three fits are the same by requiring $m_{i}=E\left(x_{i}\right)=80.45 \mathrm{GeV}(i=1,2,3)$. Once 400 groups of $x_{1}, x_{2}, x_{3}$ values are simulated, we then use (11.8) to calculate $r_{X Y}$. Take $m_{T}$ $\left(x_{1}\right)$ and $p_{T}^{\mu}\left(x_{2}\right)$ as an example, $400\left(x_{1}, x_{2}\right)$ pairs are used to obtain a $r_{1,2}$. We then repeat this process 1000 times to get a distribution of $r_{12}$, as illustrated in Figure J.2 (a). The standard deviation of the Gaussian fit gives an estimate of the error on the correlation coefficient between $x_{1}$ and $x_{2}$. The same procedure is adopted to estimate the errors on other correlation coefficients (see Figure J.2 (b) and (c)). Table 11.6 summarizes simulated errors on correlation coefficients and compares them with the errors calculated using Fisher's $z$ transformation. We conclude the obtained statistical errors on correlation coefficients using Fisher's $z$ transformation are reliable.

\subsection{Combined Mass Result}

Table 11.7 summarizes the mass fits from the three kinematic fits using $W \rightarrow \mu \nu$ sample. We use the Best-Linear -Unbiased-Estimator (BLUE) method [91] to combine the different $W$ mass fits. From uncertainties quoted in Tables 11.1, 11.2 and 
Table 11.6: Comparison of two methods to estimate errors on correlation coefficients between $m_{T}, p_{T}^{\mu}$ and $p_{T}^{\nu}$ fits in $W \rightarrow \mu \nu$ channel.

\begin{tabular}{lcc}
\hline \hline$W \rightarrow \mu \nu$ fit variable & Error on $r$ (Fisher) & Error on $r(\mathrm{MC})$ \\
\hline$m_{T}$ vs. $p_{T}^{\mu}$ & 0.028 & 0.027 \\
$m_{T}$ vs. $p_{T}^{\nu}$ & 0.028 & 0.027 \\
$p_{T}^{\mu}$ vs. $p_{T}^{\nu}$ & 0.047 & 0.043 \\
\hline \hline
\end{tabular}

Table 11.7: Muon channel $W$ mass fit results and uncertainties from $m_{T}, p_{T}^{\mu}$ and $p_{T}^{\nu}$ distributions. The fit windows are $65-90 \mathrm{GeV} / \mathrm{c}^{2}$ for the $m_{T}$ fit and $32-48 \mathrm{GeV} / \mathrm{c}$ for the $p_{T}^{\mu}$ and $p_{T}^{\nu}$ fits.

\begin{tabular}{lcc}
\hline \hline Kinematic Distribution & Result $\left(\mathrm{MeV} / \mathrm{c}^{2}\right)$ & $\chi^{2} / \mathrm{DoF}$ \\
\hline Transverse mass $m_{T}$ & $80379 \pm 16_{\text {stat }} \pm 16_{\text {syst }}$ & $57 / 48$ \\
Transverse momentum $p_{T}^{\mu}$ & $80348 \pm 18_{\text {stat }} \pm 18_{\text {syst }}$ & $58 / 62$ \\
Transverse missing momentum $p_{T}^{\nu}$ & $80406 \pm 22_{\text {stat }} \pm 20_{\text {syst }}$ & $82 / 62$ \\
\hline \hline
\end{tabular}

11.3, together with the measured statistical correlation coefficients between $m_{T}, p_{T}^{\mu}$ and $p_{T}^{\nu}$ pairs, we can get the combined mass value.

The combination of the $m_{T}$ and $p_{T}^{\mu}$ fits gives

$$
m_{W}=80369 \pm 22 \mathrm{MeV} / c^{2}
$$

This combination yields $\chi^{2} / d f=2.8 / 1$, leading to a $p$-value of $10 \%$.

The combination of the $m_{T}^{\mu}$ and $p_{T}^{\nu}$ fits gives

$$
m_{W}=80383 \pm 22 \mathrm{MeV} / c^{2}
$$

This combination yields $\chi^{2} / d f=1.4 / 1$, leading to a $p$-value of $23 \%$.

The combination of the $p_{T}^{\mu}$ and $p_{T}^{\nu}$ fits gives

$$
m_{W}=80369 \pm 23 \mathrm{MeV} / c^{2}
$$

This combination yields $\chi^{2} / d f=4.0 / 1$, leading to a $p$-value of $5 \%$. 
The combination of the $m_{T}, p_{T}^{\mu}, p_{T}^{\nu}$ fits in the muon channel gives:

$$
m_{W}=80374 \pm 22 \mathrm{MeV} / c^{2}
$$

This yields $\chi^{2} / d f=4.3 / 2$, leading to a $p$-value of $12 \%$. The relative weights of the fits are $54.5 \%, 30.6 \%, 14.9 \%$ for the $m_{T}, p_{T}^{\mu}$ and $p_{T}^{\nu}$ distributions, respectively. This final combination reduces the total uncertainy by $0.2 \mathrm{MeV}, 0.7 \mathrm{MeV}$ and $1.4 \mathrm{MeV}$ compared with the individual $m_{T}, p_{T}^{\mu}$ and $p_{T}^{\nu}$ result. Figure 11.8 compares $m_{T}, p_{T}^{\mu}$ and $p_{T}^{\nu}$ fits with the combined result. The width of the grey band is twice the total uncertainty of the combined result.

This muon channel measurement, when combined with previous Tevatron Run I and Run II measurements, will lead to a new combined Tevatron measurement of $m_{W}=80380 \pm 20 \mathrm{MeV}$. When it is further combined with LEP results, we will get a new world combined $W$ mass precision of $17 \mathrm{MeV}\left(m_{W}=80379 \pm 17 \mathrm{MeV}\right)$, which is a significant $(\sim 26 \%)$ improvement from the current world average of $23 \mathrm{MeV}$.

When the electron channel $m_{W}$ result from CDF (80406 $\pm 25 \mathrm{MeV}$ [93]) is combined with this muon channel $m_{W}$ result, we get a new CDF Run II average of $m_{W}$ $=80387 \pm 19 \mathrm{MeV}$. This leads to a new Tevatron average of $m_{W}=80393 \pm 18$ $\mathrm{MeV}$ and a new world average $m_{W}=80389 \pm 16 \mathrm{MeV}$ [93]. This is the world's most precise measurement of the $W$ boson mass. With this new world average of $m_{W}$, the estimate of the Higgs boson mass is further constrained to $m_{H}=86_{-23}^{+29} \mathrm{GeV}$. Taking into account the theory errors, this suggests $m_{H}<141 \mathrm{GeV}$ at 95\% CL [95]. 

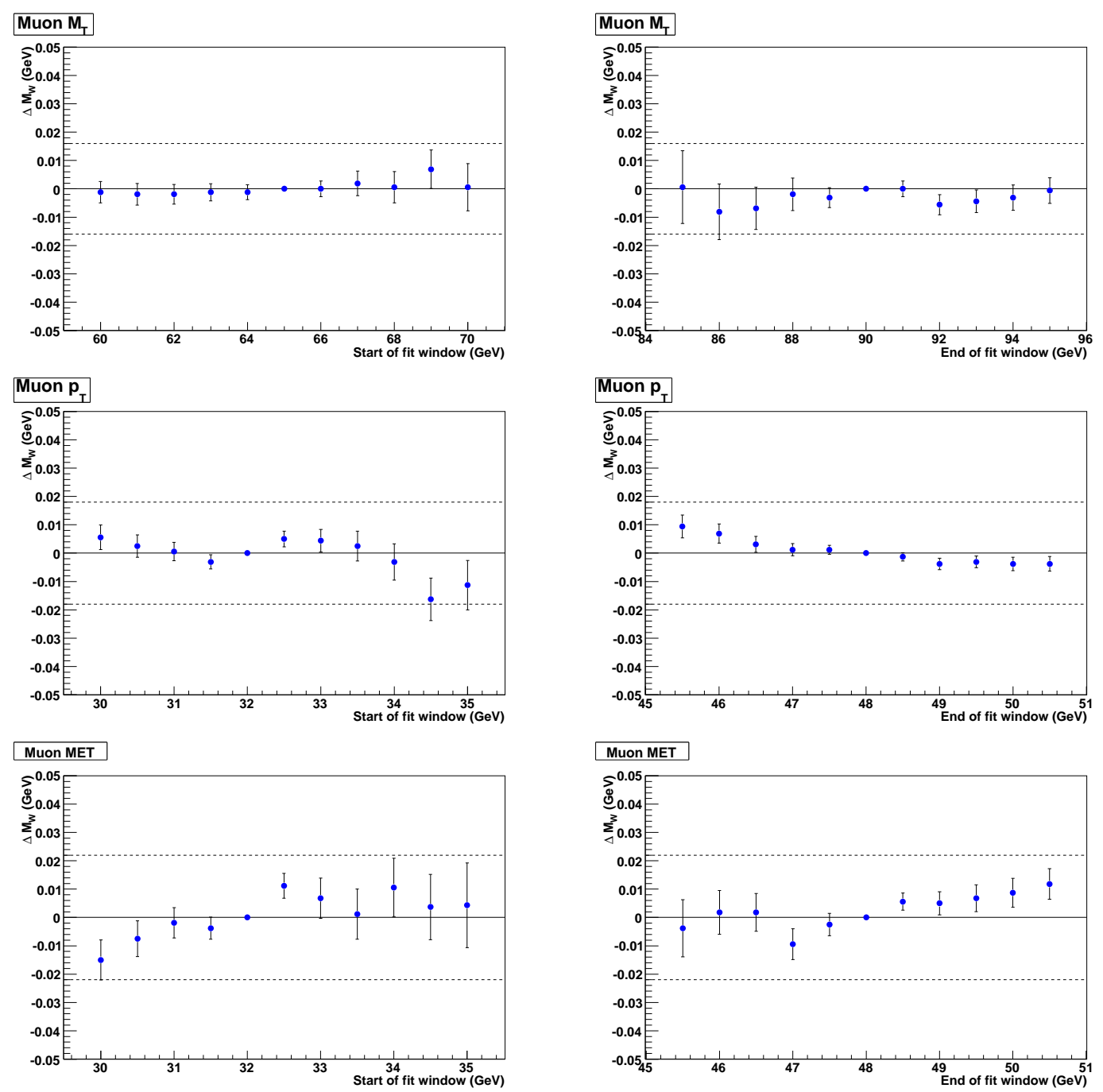

FiguRE 11.5: The shifts in measured $m_{W}$, from $W \rightarrow \mu \nu$ channel, for variations in the lower (left) and upper (right) edges of $m_{T}, p_{T}$ and $\not p_{T}$ fit ranges. The points describe the shifts in $m_{W}$ relative to the default values. The error bars are the expected one standard deviations obtained from simulation pseudoexperiments for the corresponding fitting window. 


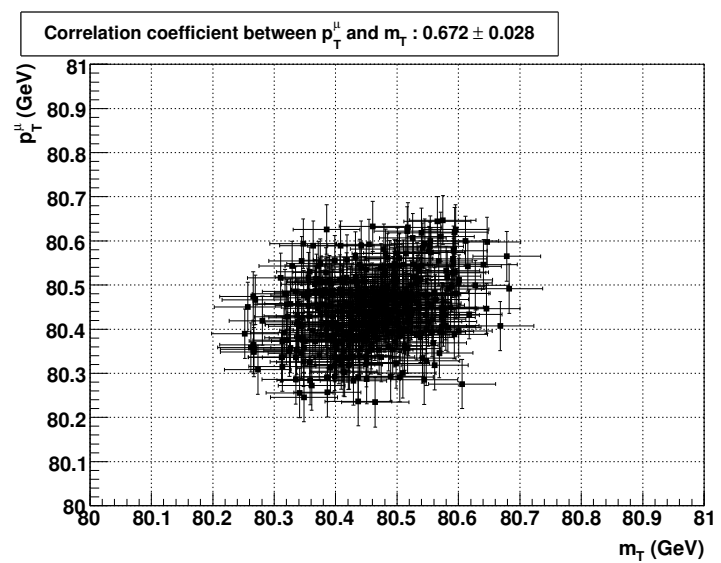

(a) Correlation between $m_{T}$ and $p_{T}^{\mu}$ fits.

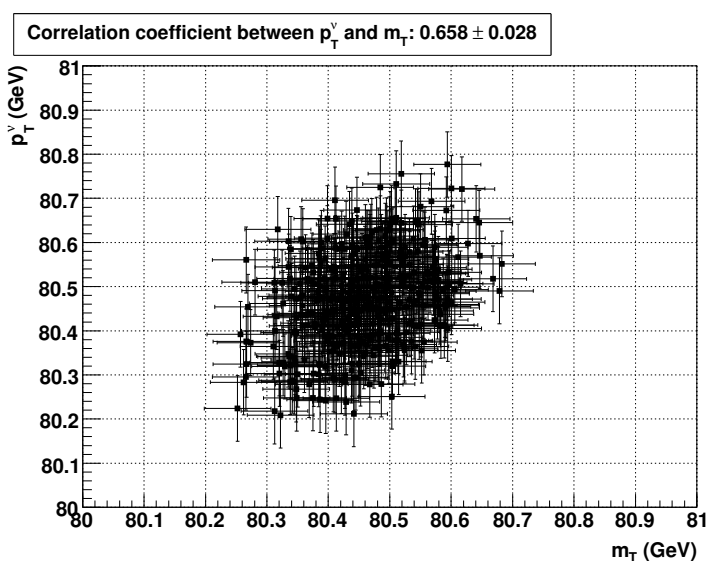

(b) Correlation between $m_{T}$ and $p_{T}^{\nu}$ fits.

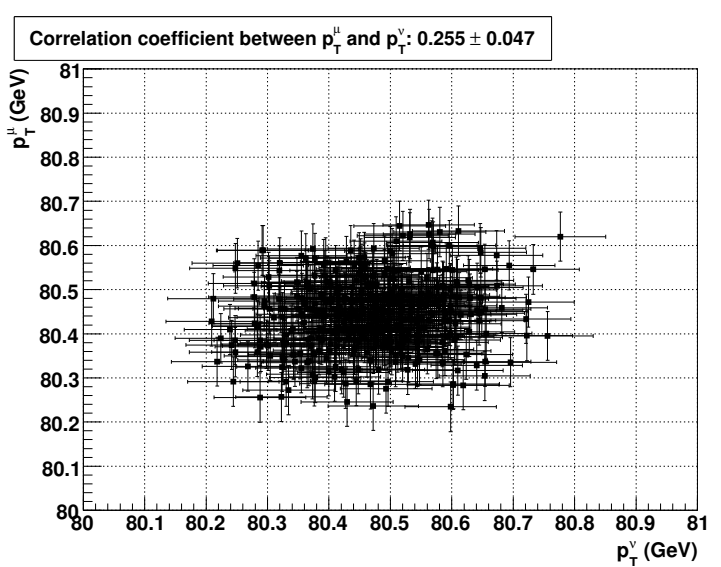

(c) Correlation between $p_{T}^{\mu}$ and $p_{T}^{\nu}$ fits.

Figure 11.6: Correlations between $m_{T}, p_{T}^{\mu}$ and $p_{T}^{\nu}$ fits. 


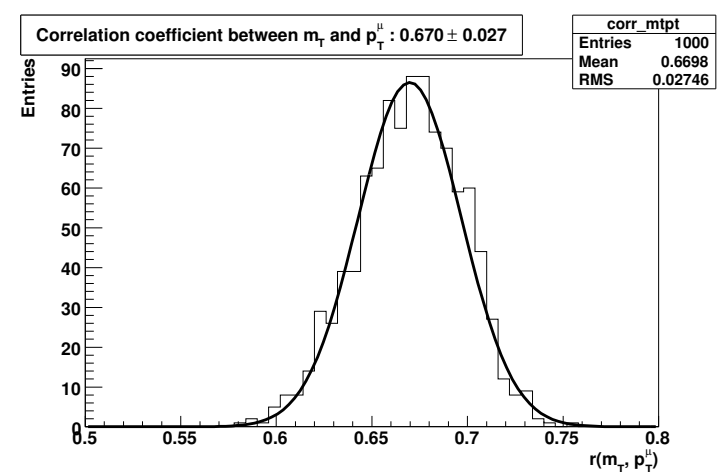

(a) Error on $m_{T}$-and- $p_{T}^{\mu}$ correlation coefficient.

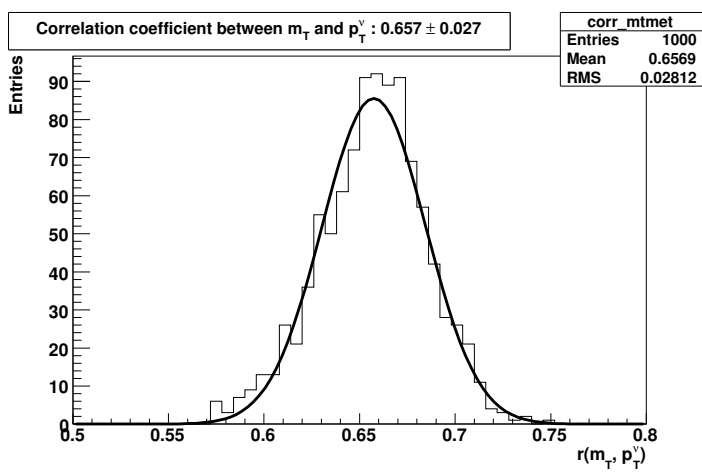

(b) Error on $m_{T}$-and- $p_{T}^{\nu}$ correlation coefficient.

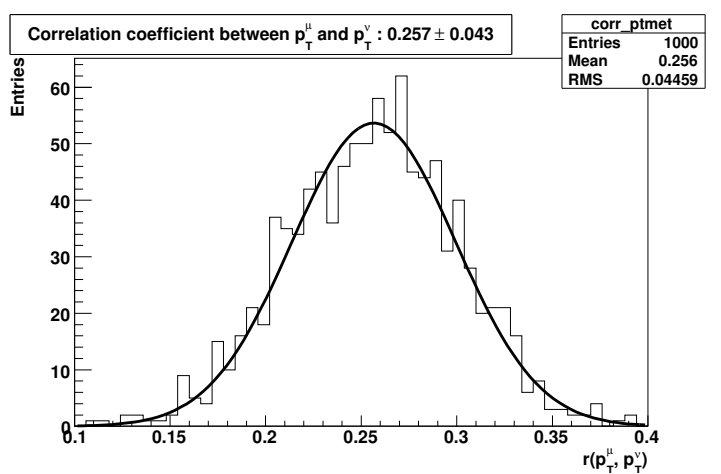

(c) Error on $p_{T}^{\mu}$-and- $p_{T}^{\nu}$ correlation coefficient.

Figure 11.7: Monte Carlo study of the errors on correlation coefficients between $m_{T}, p_{T}^{\mu}$ and $p_{T}^{\nu}$ fits. 


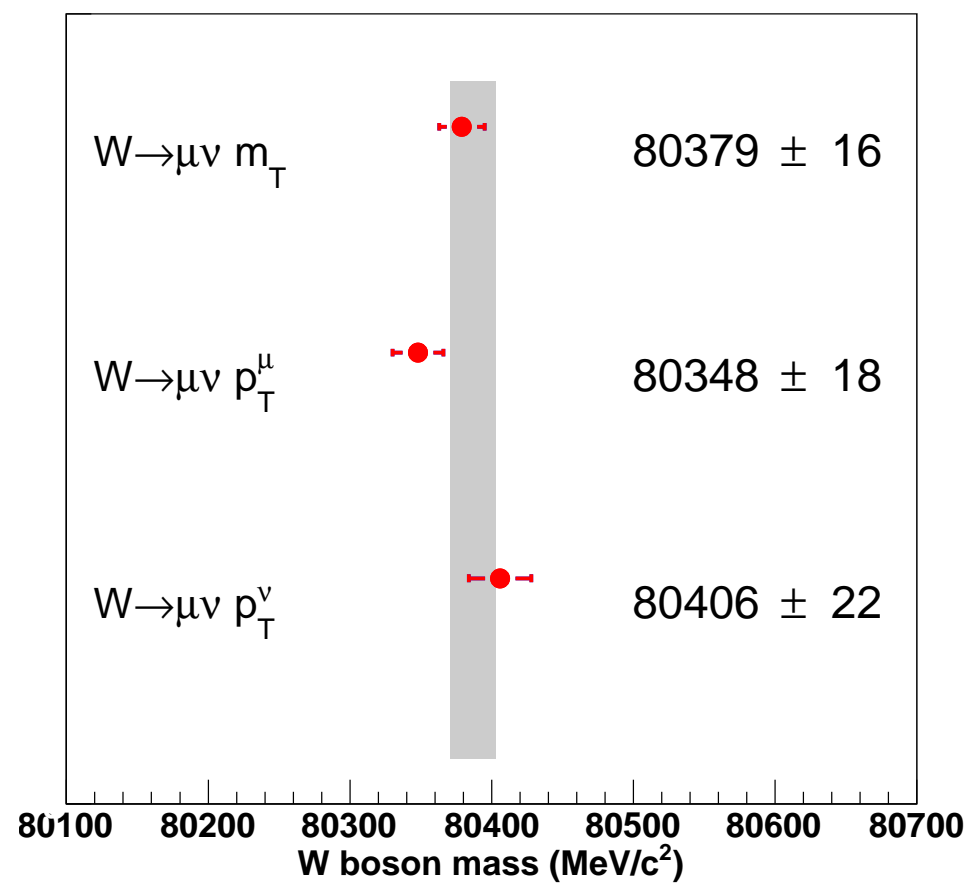

Figure 11.8: Comparison of the $W$ boson mass measurement from $m_{T}, p_{T}^{\mu}$ and $p_{T}^{\nu}$ fits. The uncertainties are statistical only. The dark band is for the new worldaverage $m_{W}$ and its associated total uncertainty. 
12

\section{Summary and Future Pespective}

\subsection{Summary}

In this thesis, we have analyzed $2.2 \mathrm{fb}^{-1}$ data collected by the CDF detector between Febuary 2002 to August 2007. The measured $W$ boson mass from the muon decaying channel is found to be:

$$
\begin{array}{cc}
m_{W}\left(m_{T}\right)=80379 \pm 16_{\text {stat }} \pm 16_{\text {syst }} \mathrm{MeV} / \mathrm{c}^{2} \\
m_{W}\left(p_{T}^{\mu}\right)=80348 \pm 18_{\text {stat }} \pm 18_{\text {syst }} \mathrm{MeV} / \mathrm{c}^{2} \\
m_{W}\left(p_{T}^{\nu}\right)=80406 \pm 22_{\text {stat }} \pm 20_{\text {syst }} \mathrm{MeV} / \mathrm{c}^{2}
\end{array}
$$

This leads to a combined $W$ mass from the muon channel to be [91]:

$$
m_{W}=80374 \pm 15(\text { stat }) \pm 16(\text { syst })=80374 \pm 22 \mathrm{MeV} / \mathrm{c}^{2}
$$

The relative weights of the fits are $54.5 \%, 30.6 \%, 14.9 \%$ for the $m_{T}, p_{T}^{\mu}$ and $p_{T}^{\nu}$

distributions. The combination yields a $\chi^{2} /$ dof of $4.3 / 2$, leading to a $p$-value of $12 \%$. This muon channel measurement alone is already more precise than the current world-average $m_{W}$, which has a precision of $23 \mathrm{MeV}$. 
When the electron channel $m_{W}$ result from $\mathrm{CDF}(80406 \pm 25 \mathrm{MeV}$ [93]) is combined with this muon channel $m_{W}$ result, we get a new CDF Run II average of $m_{W}^{\mathrm{CDF}-\mathrm{II}}=80387 \pm 19 \mathrm{MeV}$, with $62 \%$ (38\%) contribution from the muon (electron) channel. This leads to a new Tevatron average of $m_{W}^{\text {Tevatron }}=80393 \pm 18 \mathrm{MeV}$ and a new world average $m_{W}^{\text {world-average }}=80389 \pm 16 \mathrm{MeV}$ [93]. The uncertainty on the world average value is thus improved by $30 \%$. With this new world average of $m_{W}$, the Higgs boson mass is further constrained to $m_{H}=86_{-23}^{+29} \mathrm{GeV}$ [95]. When theory errors are taken into account, we find $m_{H}<141 \mathrm{GeV}$ at $95 \% \mathrm{CL}$ [95].

Figure 12.1 compares previous measurements of the $W$ boson mass with our electron/muon channel combined CDF measurement. Our measurement result is significantly better than all previous measurements. Recently, D $\varnothing$ has measured $m_{W}=80367 \pm 26 \mathrm{MeV}$ using $4.3 \mathrm{fb}^{-1}$ data collected by $\mathrm{D} \varnothing$ detector. When this $4.3 \mathrm{fb}^{-1} \mathrm{D} \varnothing$ result is combined with an earlier $\mathrm{D} \varnothing m_{W}$ measurement using $1 \mathrm{fb}^{-1}$ data, D $\varnothing$ obtains $m_{W}=80375 \pm 23 \mathrm{MeV}$. Combining the latest $\mathrm{CDF}$ and $\mathrm{D} \varnothing m_{W}$ measurements with previous LEP and Teveratron Run I measurements, the worldaverage precision of $m_{W}$ is significantly reduced from $23 \mathrm{MeV}$ to $15 \mathrm{MeV}$, and most of the weight $(60.3 \%$ [96]) comes from our latest CDF measurement. Figure 12.2 shows the new $m_{W}-m_{t}$ plot with the latest CDF and D $\varnothing m_{W}$ measurements. We can see from Figure 12.2 that if light Higgs is found, then $m_{W}$ provides a precise confirmation of the SM; if light Higgs is excluded, then it will be a $>5 \sigma$ exclusion of the SM and definitively points to new physics beyond the SM.

\subsection{Future Perspective}

CDF has started the a new $W$ mass analysis with the final CDF dataset $\left(\sim 10 \mathrm{fb}^{-1}\right)$ and $\mathrm{D} \varnothing$ is expected to start their final $W$ mass with the final $\mathrm{D} \varnothing$ dataset too. The $W$ mass measurement precision from the Tevatron is expected to be further improved to $\sim 10 \mathrm{MeV}$. See Figure 12.3 for the future projection of $W$ mass precision at Tevatron 


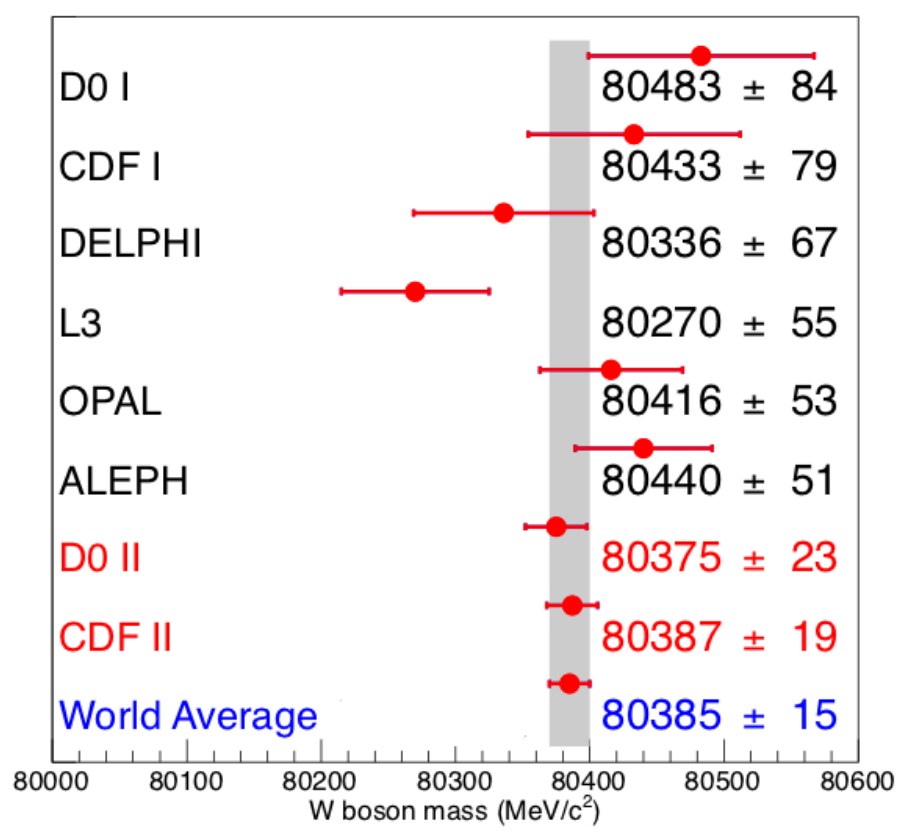

Figure 12.1: Recent and past $W$ boson mass measurements. Combining muon channel result with electron channel result, CDF reaches a precision of $19 \mathrm{MeV}$, which is significantly better than all previous measurements.

by $\mathrm{CDF}$ and $\mathrm{D} \varnothing$ experiments.

In the long term, with large data sample collected, LHC may have the potential to measure $m_{W}$ with a precision of $5 \sim 10 \mathrm{MeV}$. An exciting possibility of $W$ boson mass measurement is that the predicted Higgs boson mass might disagree with existing bounds from direct searches, or it might disagree with the measured mass of the Higgs boson after it is discovered at the Large Hadron Collider. In either case, the $W$ boson mass measurement would pave the way to new physics beyond the Higgs theory. 


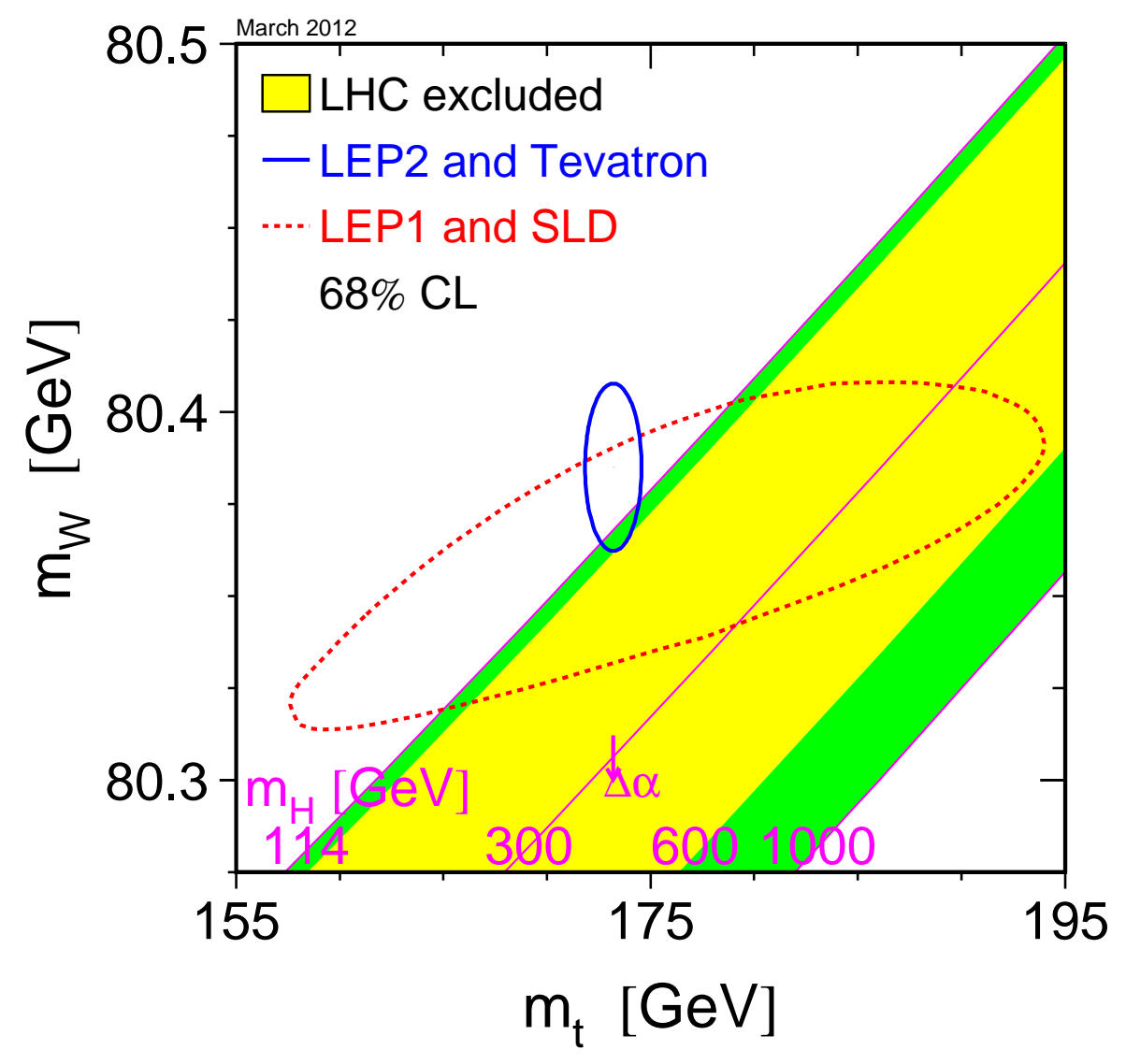

Figure 12.2: Updated $m_{W}$ vs. $m_{t}$ plot with the latest CDF and D $\varnothing m_{W}$ measurements. The green region is SM-allowed region and the yellow region has been excluded by LHC experiments. 


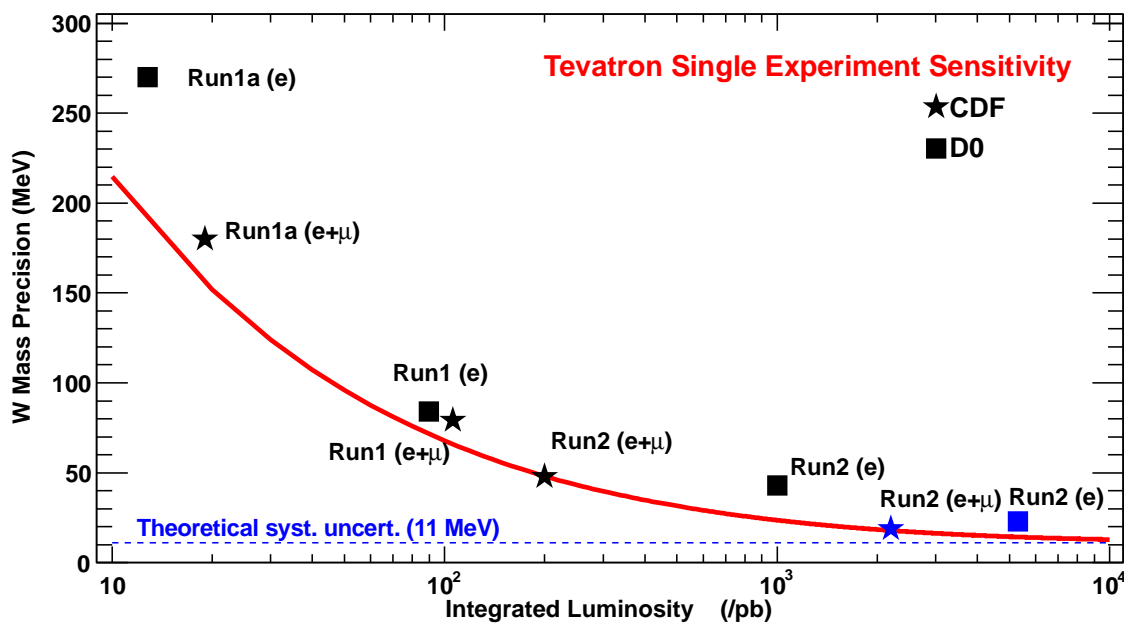

Figure 12.3: Projection of measurement precision of the $W$ boson mass at CDF and $\mathrm{D} \varnothing$ as a function of integrated luminosity $\mathcal{L}$. The red curve is obtained by assuming the theoretical uncertainties from PDF $(10 \mathrm{MeV})$ and QED (4 MeV) remain unchanged, while other systematic uncertainties and statistical uncertainy scale as $1 / \sqrt{\mathcal{L}}$. 


\section{Appendix A}

\section{PDF Uncertainties in $W \rightarrow e \nu$ channel}

The same procedure is adopted in studying PDF uncertainties in $W \rightarrow e \nu$ channel. The variations of fitted $m_{W}$ difference from the default CTEQ6M are shown in Figures A.1 A.3.

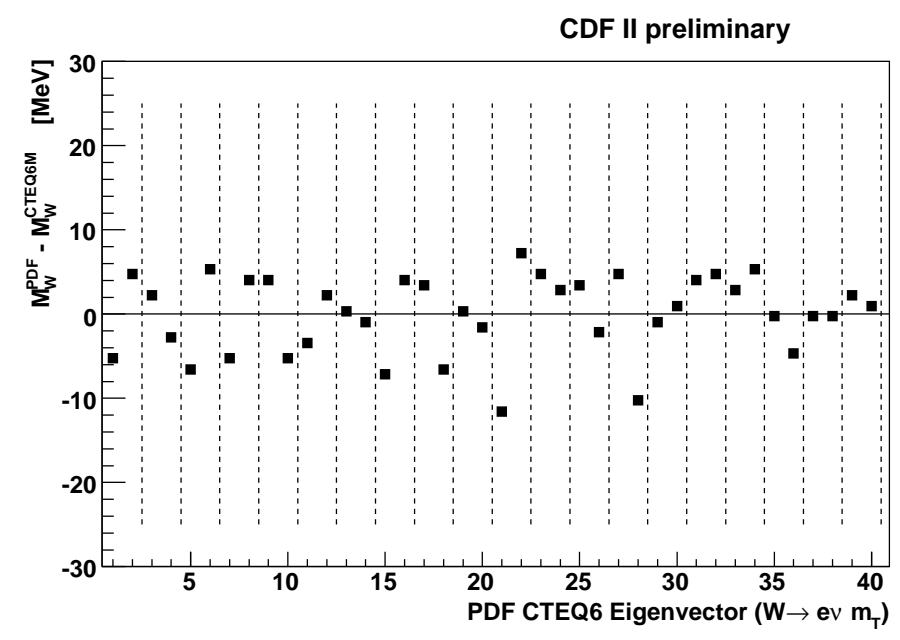

Figure A.1: Variations of fitted $m_{W}$ difference from the default using $W \rightarrow e \nu m_{T}$ distributions over 20 pairs of CTEQ6 error PDFs. The difference in $m_{W}$ is between each error PDF and the default CTEQ6M. 


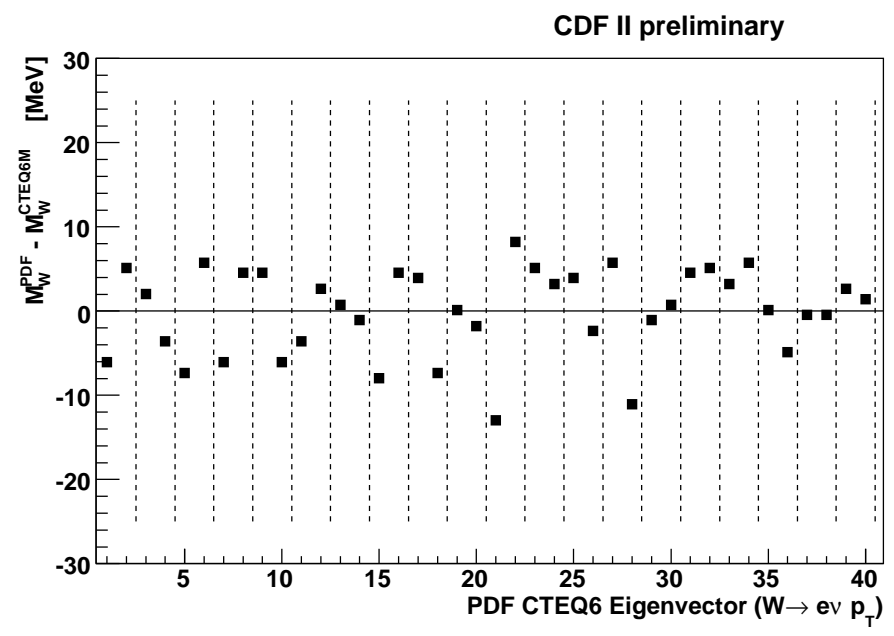

Figure A.2: Variations of fitted $m_{W}$ difference from the default using $W \rightarrow e \nu p_{T}^{e}$ distributions over 20 pairs of CTEQ6 error PDFs. The difference in $m_{W}$ is between each error PDF and the default CTEQ6M.

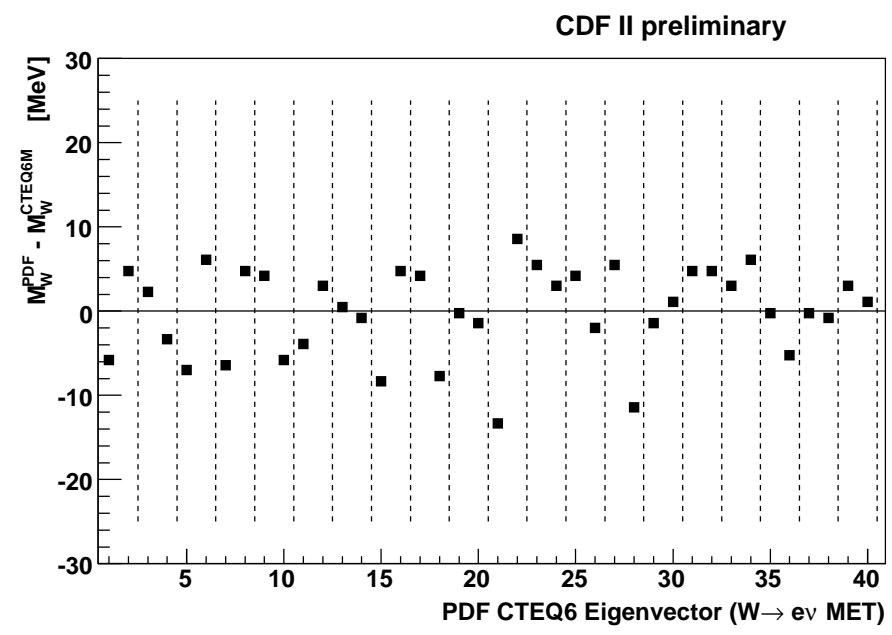

Figure A.3: Variations of fitted $m_{W}$ difference from the default using $W \rightarrow e \nu E_{T}$ distributions over 20 paris of CTEQ6 error PDFs. The difference in $m_{W}$ is between each error PDF and the default CTEQ6M. 


\section{Appendix B}

\section{$Z / \gamma$ interference}

Since the standard $Z$ boson generation with REsBos does not include the $Z / \gamma$ interference contribution, we have to take this effect into account and add it to the $Z$-pole distribution. This is done by reweighting the reconstructed mass of $Z \rightarrow l l$ events in DukeSim. One way to determine the weights is to use PYTHIA event generator, which has the option to run in three different modes: $Z$-pole only (mode 1), Drell-Yan only (mode 2) and $Z$-pole plus Drell-Yan with interference (mode 3).

We generate 100 million $Z \rightarrow \mu \mu$ PYTHIA events for each mode while requiring the $Z$ mass to be within $70 \mathrm{GeV}$ and $110 \mathrm{GeV}$. In this study, the ISR, the FSR and the fragmentation options in PYTHIA event generation are turned off. If we subtract the reconstructed invariant mass spectrum from "mode $1+$ mode 2 " from the reconstructed invariant mass spectrum from mode 3 and normalize it to mode 1 , we can get the $Z / \gamma$ interference contribution to the $Z$ production. This is illustrated in Figure B.1. 


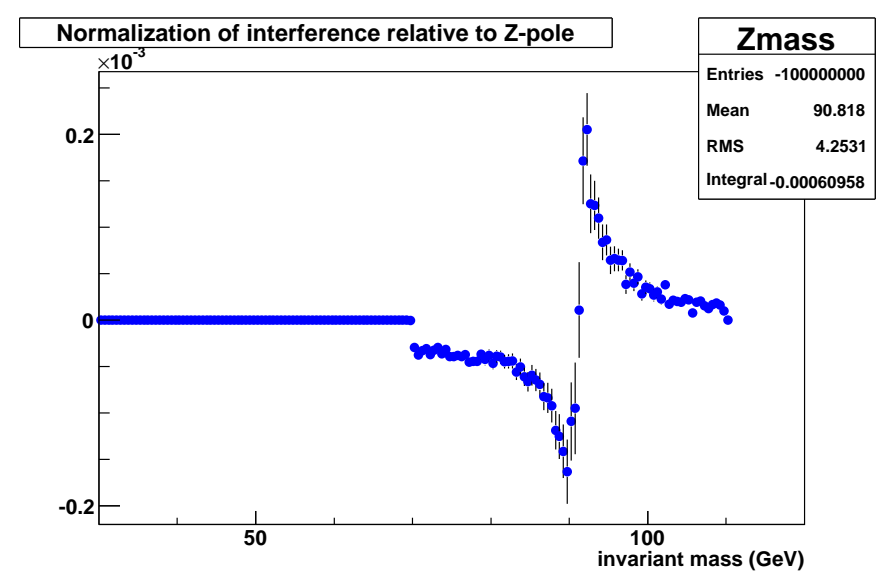

FiguRE B.1: $Z / \gamma$ interference contribution to the $Z$ production from RESBOS. 


\section{Appendix $\mathrm{C}$}

\section{Relationship between $\Delta p / p$ and $\left\langle 1 / p_{T}^{\mu}\right\rangle$}

The $J / \psi \rightarrow \mu \mu$ invariant mass is given by:

$$
\begin{aligned}
m_{J / \psi} & =\sqrt{\left(E_{\mu^{+}}+E_{\mu^{-}}\right)^{2}-\left(\vec{p}_{\mu^{+}}+\vec{p}_{\mu^{-}}\right)^{2}} \\
& \approx \sqrt{4 E_{\mu^{+}} E_{\mu^{-}}}
\end{aligned}
$$

Assume $\mu^{ \pm}$track experiences an energy loss of $E_{I}^{\mu^{ \pm}}\left(E_{I}^{\mu^{+}} \approx E_{I}^{\mu^{-}}=E_{I}\right)$, we have the invariant mass of $J / \psi \rightarrow \mu \mu$ events to be:

$$
\begin{aligned}
m_{J / \psi}^{\prime} & \approx \sqrt{4 E_{\mu^{+}} E_{\mu^{-}}} \\
& =\sqrt{4\left(E_{\mu^{+}} E_{\mu^{-}}-E_{\mu^{+}} E_{I}^{\mu^{-}}-E_{\mu^{-}} E_{I}^{\mu^{+}}+E_{I}^{\mu^{+}} E_{I}^{\mu^{-}}\right)}
\end{aligned}
$$


We then have:

$$
\begin{aligned}
\frac{\Delta m}{m} & =\frac{m_{J / \psi}^{\prime}-m_{J / \psi}}{m_{J / \psi}} \\
& =\sqrt{1-\frac{E_{I}^{\mu^{-}}}{E_{\mu^{-}}}-\frac{E_{I}^{\mu^{+}}}{E_{\mu^{+}}}+\frac{E_{I}^{\mu^{+}} E_{I}^{\mu^{-}}}{E_{\mu^{+}} E_{\mu^{-}}}}-1 \\
& \approx \sqrt{1-\left(\frac{E_{I}^{\mu^{-}}}{E_{\mu^{-}}}+\frac{E_{I}^{\mu^{+}}}{E_{\mu^{+}}}\right)}-1 \\
& \approx-\frac{1}{2}\left(\frac{E_{I}^{\mu^{-}}}{E_{\mu^{-}}}+\frac{E_{I}^{\mu^{+}}}{E_{\mu^{+}}}\right) \\
& \approx-\frac{E_{I}}{2}\left(\frac{1}{p_{\mu^{+}}}+\frac{1}{p_{\mu^{-}}}\right) \\
& \approx-E_{I}\left\langle 1 / p_{T}^{\mu}\right\rangle
\end{aligned}
$$

Since $\Delta m / m \approx \Delta p / p$, we can see $\Delta p / p$ is approximately a linear function of $\left\langle 1 / p_{T}^{\mu}\right\rangle$ and the slope of this linear dependence approximately corresponds to ionization energy loss $E_{I}$. Since we model this ionization energy loss based on the known knowledge of detector material, this slope should be zero. We find we need to scale up our material description by $4.3 \%$ to achieve a zero slope. 


\section{Appendix D}

\section{Individual $J / \psi$ Fits in $\left\langle 1 / p_{T}^{\mu}\right\rangle$ Bins}

Fitting results to $J / \psi \rightarrow \mu \mu$ invarariant mass spectra in $15\left\langle 1 / p_{T}^{\mu}\right\rangle$ bins are shown in Figures D.1 to D.15. Each of the fitted $\Delta p / p$ result contributes one entry to Figure 8.18. The background in each invariant mass fit is linearly parametrized with the slope and the intercept determined by minimizing the $\chi^{2}$ in a fit range centering at the mass peak which is twice as wide as the nominal fitting window. The size of the nominal fitting window is adjusted according to the mass resolution for different $\left\langle 1 / p_{T}^{\mu}\right\rangle$ bins.

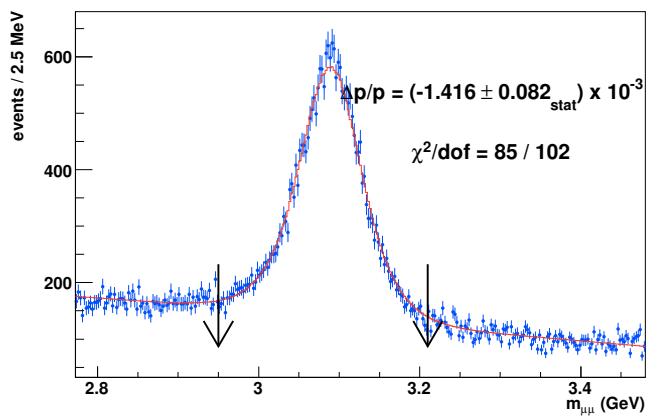

Figure D.1: Fitted $\Delta p / p$ of the first $\left\langle 1 / p_{T}^{\mu}\right\rangle$ bin in Figure 8.18 .

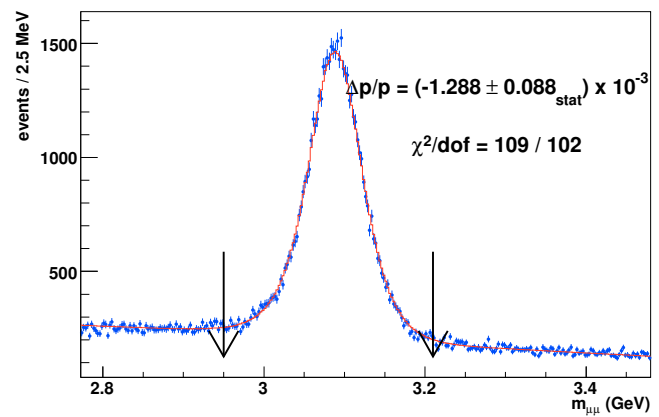

Figure D.2: Fitted $\Delta p / p$ of the second $\left\langle 1 / p_{T}^{\mu}\right\rangle$ bin in Figure 8.18. 


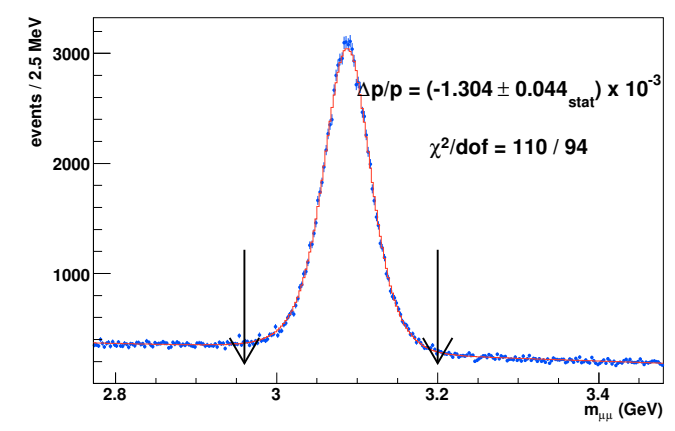

Figure D.3: Fitted $\Delta p / p$ of the third $\left\langle 1 / p_{T}^{\mu}\right\rangle$ bin in Figure 8.18.

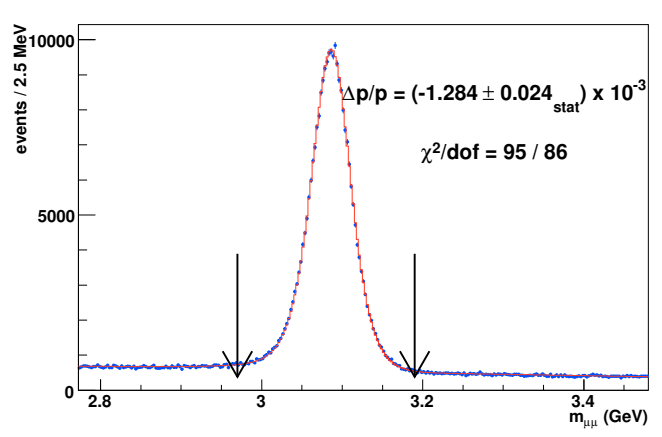

Figure D.5: Fitted $\Delta p / p$ of the fifth $\left\langle 1 / p_{T}^{\mu}\right\rangle$ bin in Figure 8.18.

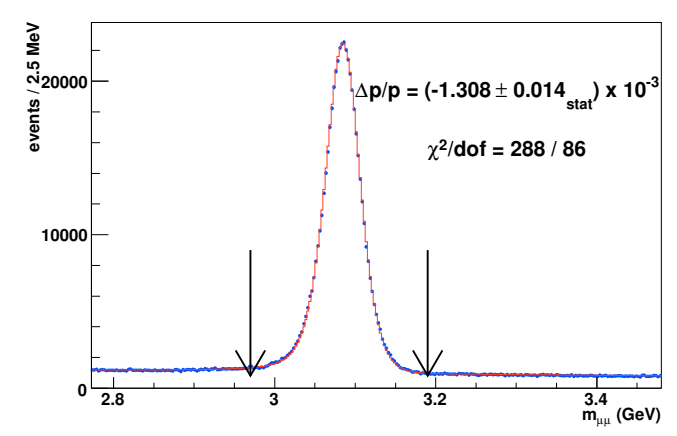

Figure D.7: Fitted $\Delta p / p$ of the seventh $\left\langle 1 / p_{T}^{\mu}\right\rangle$ bin in Figure 8.18.

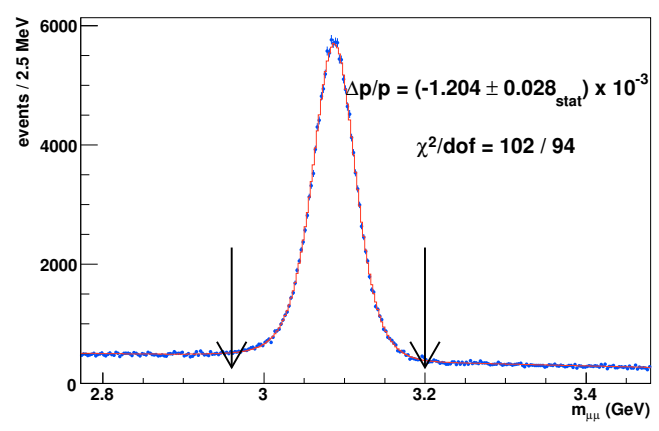

Figure D.4: Fitted $\Delta p / p$ of the fourth $\left\langle 1 / p_{T}^{\mu}\right\rangle$ bin in Figure 8.18.

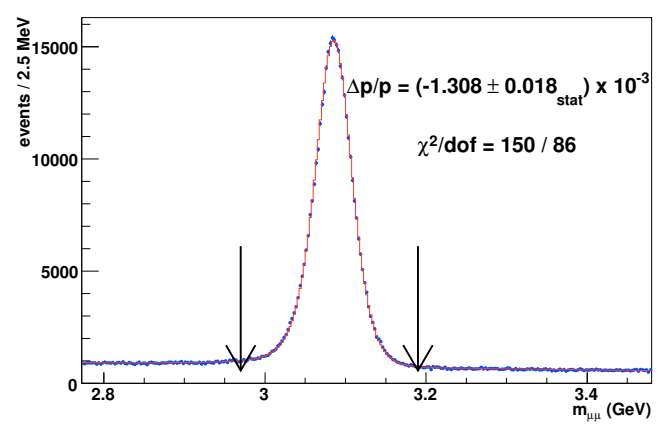

Figure D.6: Fitted $\Delta p / p$ of the sixth $\left\langle 1 / p_{T}^{\mu}\right\rangle$ bin in Figure 8.18.

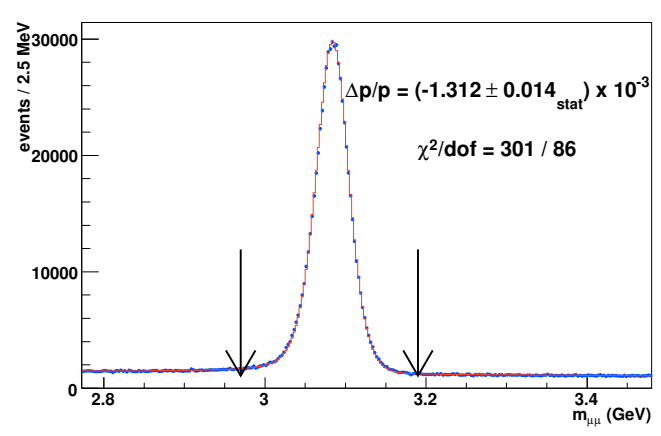

Figure D.8: Fitted $\Delta p / p$ of the eighth $\left\langle 1 / p_{T}^{\mu}\right\rangle$ bin in Figure 8.18. 


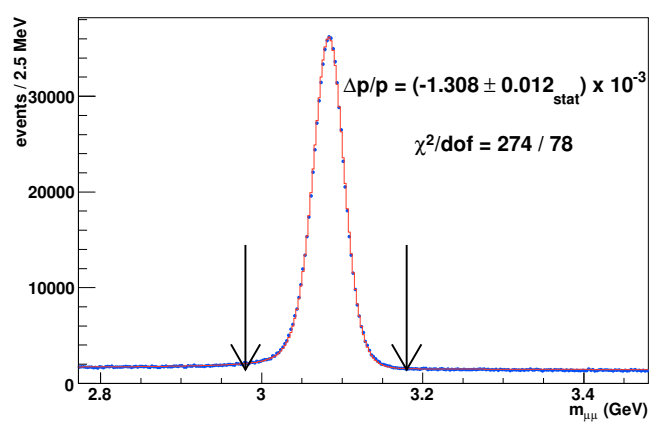

Figure D.9: Fitted $\Delta p / p$ of the ninth $\left\langle 1 / p_{T}^{\mu}\right\rangle$ bin in Figure 8.18.

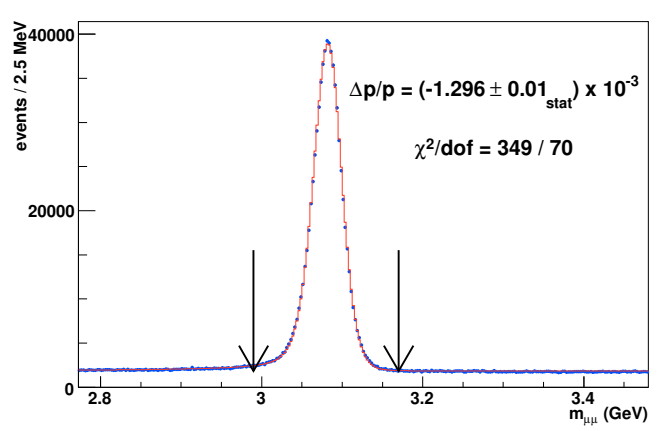

Figure D.11: Fitted $\Delta p / p$ of the eleventh $\left\langle 1 / p_{T}^{\mu}\right\rangle$ bin in Figure 8.18.

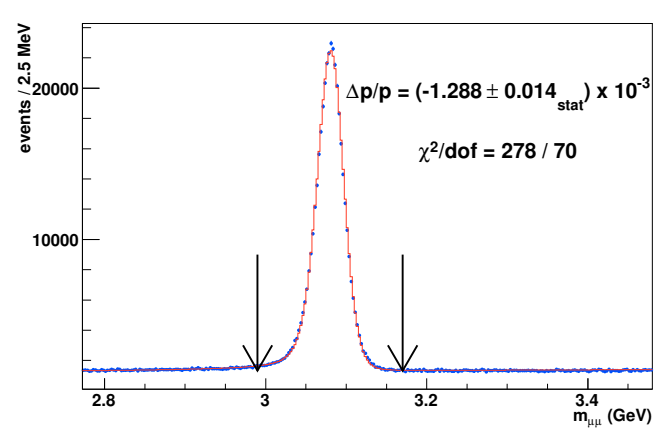

Figure D.13: Fitted $\Delta p / p$ of the thirteenth $\left\langle 1 / p_{T}^{\mu}\right\rangle$ bin in Figure 8.18.

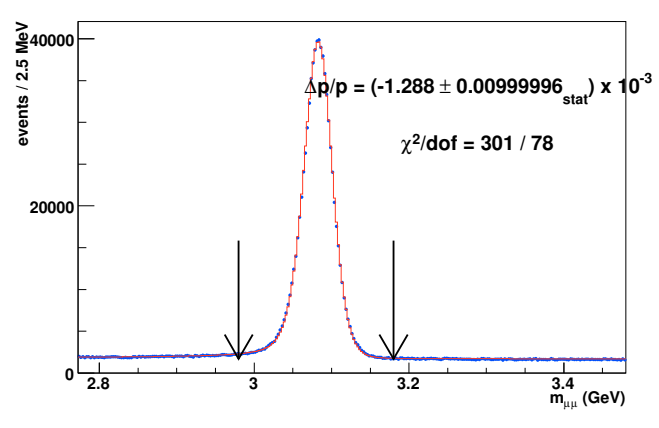

Figure D.10: Fitted $\Delta p / p$ of the tenth $\left\langle 1 / p_{T}^{\mu}\right\rangle$ bin in Figure 8.18.

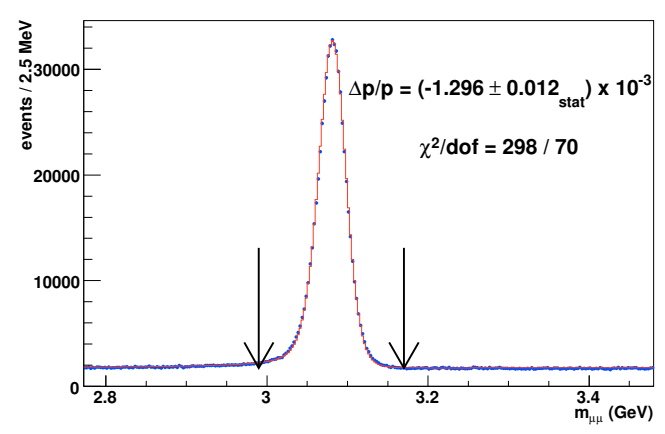

Figure D.12: Fitted $\Delta p / p$ of the twelfth $\left\langle 1 / p_{T}^{\mu}\right\rangle$ bin in Figure 8.18.

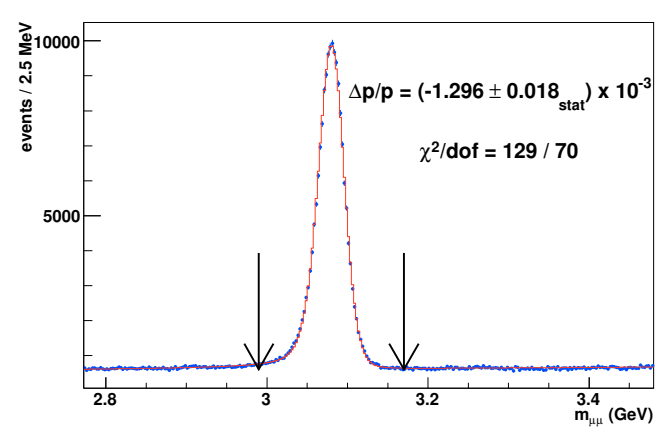

FiguRE D.14: Fitted $\Delta p / p$ of the fourteenth $\left\langle 1 / p_{T}^{\mu}\right\rangle$ bin in Figure 8.18. 


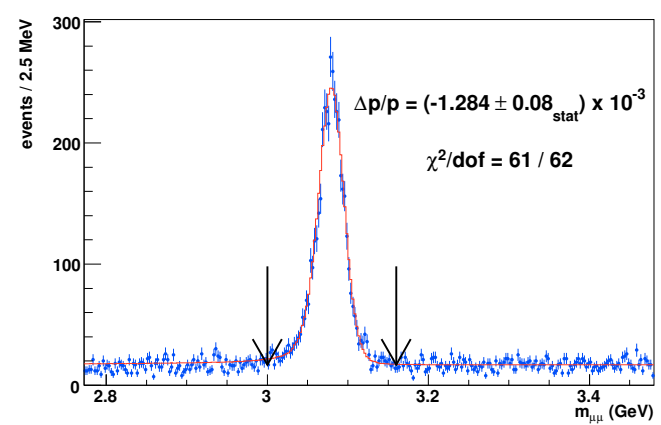

Figure D.15: Fitted $\Delta p / p$ of the fifteenth $\left\langle 1 / p_{T}^{\mu}\right\rangle$ bin in Figure 8.18. 


\section{Appendix E}

\section{Beam-constrained $\Upsilon$ Fit in $\left\langle 1 / p_{T}^{\mu}\right\rangle$ Bins}

Fitting results to beam-constrained $\Upsilon \rightarrow \mu \mu$ invarariant mass spectra in $9\left\langle 1 / p_{T}^{\mu}\right\rangle$ bins are shown in Figures E.1 to E.9. Each of the fitted $\Delta p / p$ result contributes one entry to Figure 8.18. The background in each invariant mass fit is linearly parametrized with the slope and the intercept determined by minimizing the $\chi^{2}$ in a fit window indicated by the arrows in the plots. The size of the nominal fit window is adjusted according to the mass resolution for different $\left\langle 1 / p_{T}^{\mu}\right\rangle$ bins.

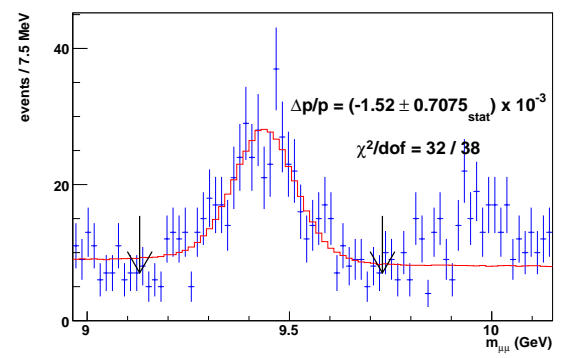

Figure E.1: Fitted $\Delta p / p$ of the first $\left\langle 1 / p_{T}^{\mu}\right\rangle$ bin in Figure 8.18 .

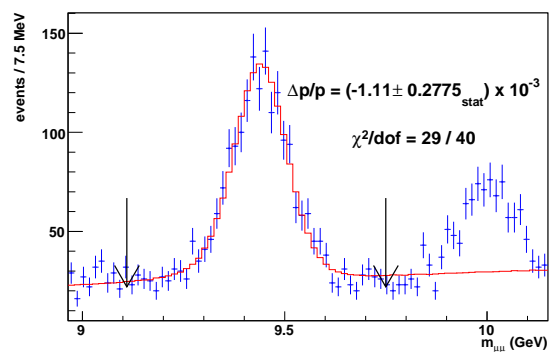

Figure E.2: Fitted $\Delta p / p$ of the second $\left\langle 1 / p_{T}^{\mu}\right\rangle$ bin in Figure 8.18. 


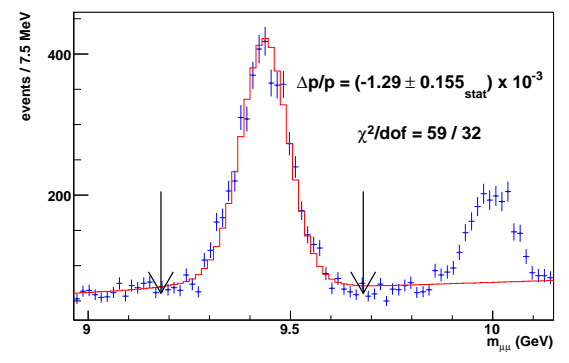

Figure E.3: Fitted $\Delta p / p$ of the third $\left\langle 1 / p_{T}^{\mu}\right\rangle$ bin in Figure 8.18.

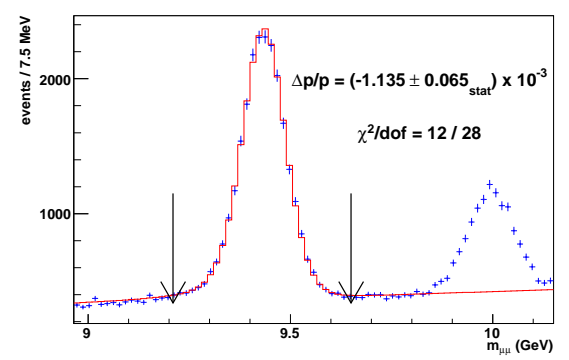

Figure E.5: Fitted $\Delta p / p$ of the fifth $\left\langle 1 / p_{T}^{\mu}\right\rangle$ bin in Figure 8.18.

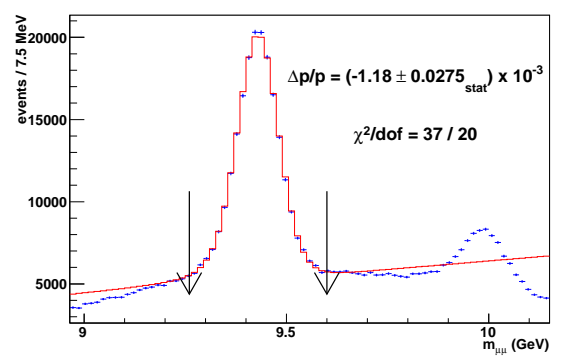

Figure E.7: Fitted $\Delta p / p$ of the seventh $\left\langle 1 / p_{T}^{\mu}\right\rangle$ bin in Figure 8.18.

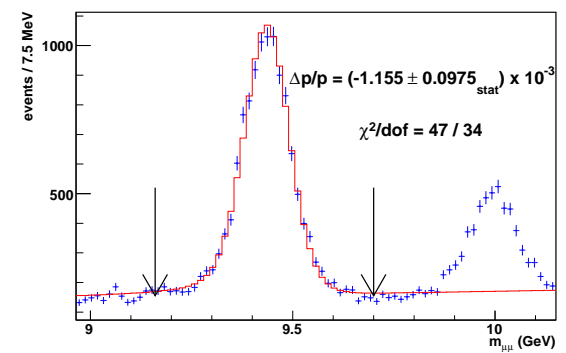

Figure E.4: Fitted $\Delta p / p$ of the fourth $\left\langle 1 / p_{T}^{\mu}\right\rangle$ bin in Figure 8.18.

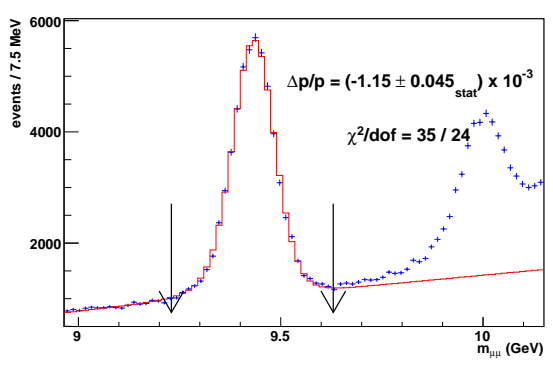

Figure E.6: Fitted $\Delta p / p$ of the sixth $\left\langle 1 / p_{T}^{\mu}\right\rangle$ bin in Figure 8.18.

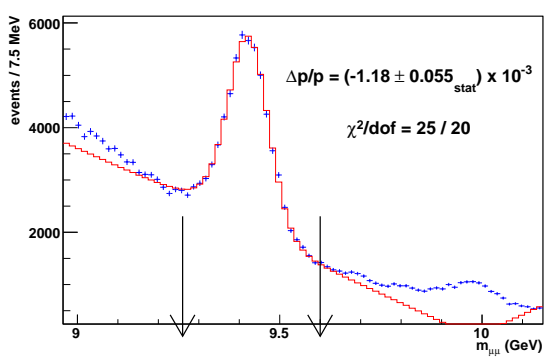

Figure E.8: Fitted $\Delta p / p$ of the eighth $\left\langle 1 / p_{T}^{\mu}\right\rangle$ bin in Figure 8.18. 


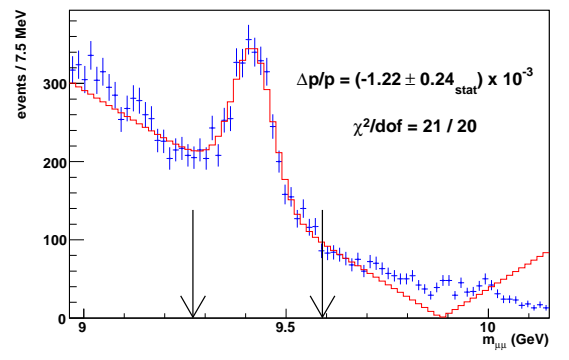

Figure E.9: Fitted $\Delta p / p$ of the ninth $\left\langle 1 / p_{T}^{\mu}\right\rangle$ bin in Figure 8.18. 


\section{Appendix F}

\section{Non-beam-constrained $\Upsilon$ Fit in $\left\langle 1 / p_{T}^{\mu}\right\rangle$ Bins}

Fitting results to non-beam-constrained $\Upsilon \rightarrow \mu \mu$ invarariant mass spectra in $9\left\langle 1 / p_{T}^{\mu}\right\rangle$ bins are shown in Figures F.1 to F.9. Each of the fitted $\Delta p / p$ result contributes one entry to Figure 8.18. The background in each invariant mass fit is linearly parametrized with the slope and the intercept determined by minimizing the $\chi^{2}$ in a fit window indicated by the arrows in the plots. The size of the nominal fit window is adjusted according to the mass resolution for different $\left\langle 1 / p_{T}^{\mu}\right\rangle$ bins.

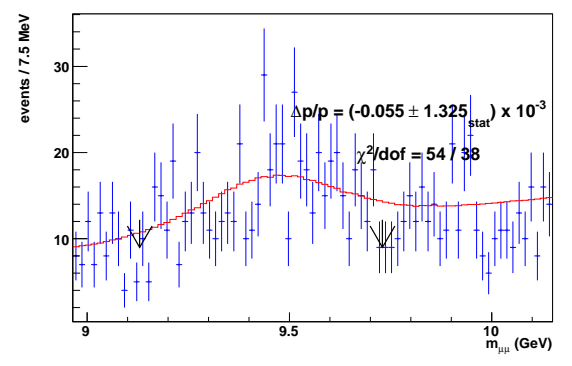

Figure F.1: Fitted $\Delta p / p$ of the first $\left\langle 1 / p_{T}^{\mu}\right\rangle$ bin in Figure 8.18 .

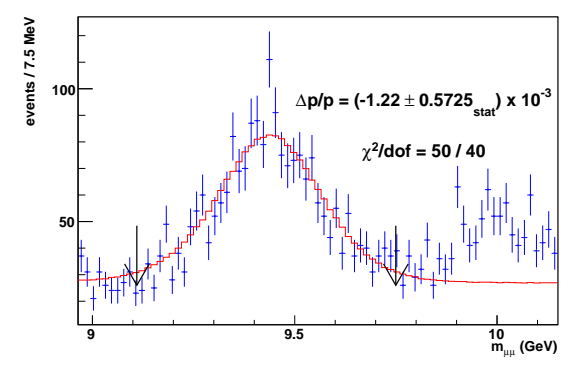

Figure F.2: Fitted $\Delta p / p$ of the second $\left\langle 1 / p_{T}^{\mu}\right\rangle$ bin in Figure 8.18. 


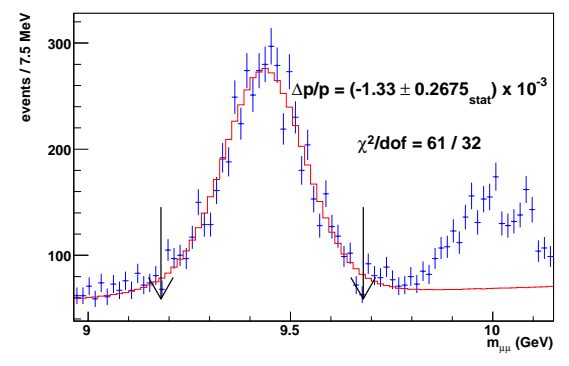

Figure F.3: Fitted $\Delta p / p$ of the third $\left\langle 1 / p_{T}^{\mu}\right\rangle$ bin in Figure 8.18.

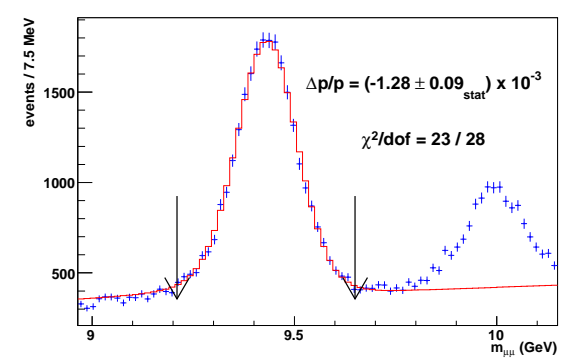

Figure F.5: Fitted $\Delta p / p$ of the fifth $\left\langle 1 / p_{T}^{\mu}\right\rangle$ bin in Figure 8.18.

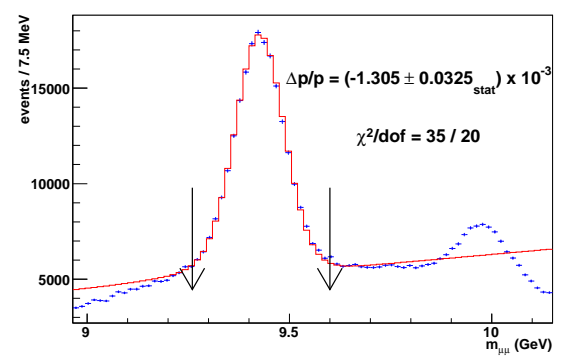

Figure F.7: Fitted $\Delta p / p$ of the seventh $\left\langle 1 / p_{T}^{\mu}\right\rangle$ bin in Figure 8.18.

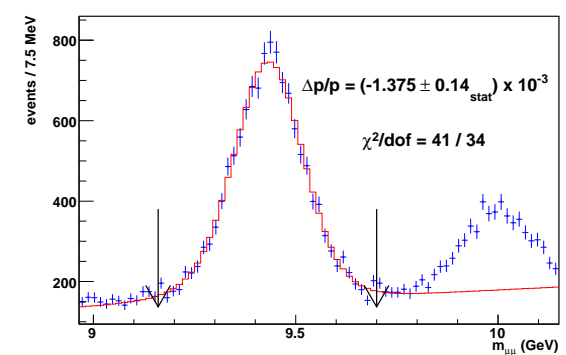

Figure F.4: Fitted $\Delta p / p$ of the fourth $\left\langle 1 / p_{T}^{\mu}\right\rangle$ bin in Figure 8.18.

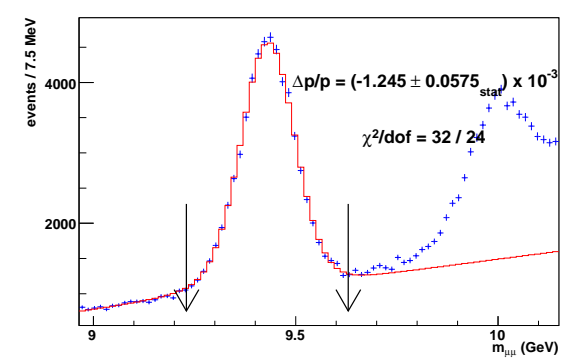

Figure F.6: Fitted $\Delta p / p$ of the sixth $\left\langle 1 / p_{T}^{\mu}\right\rangle$ bin in Figure 8.18.

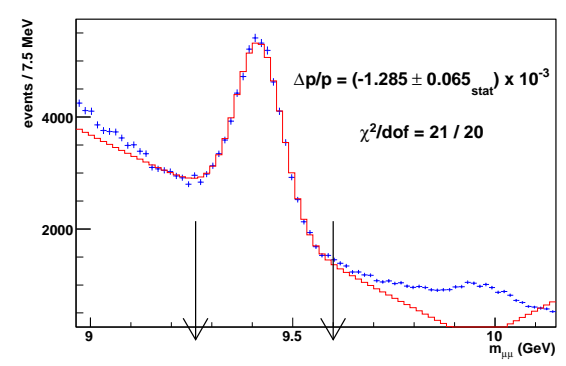

Figure F.8: Fitted $\Delta p / p$ of the eighth $\left\langle 1 / p_{T}^{\mu}\right\rangle$ bin in Figure 8.18. 


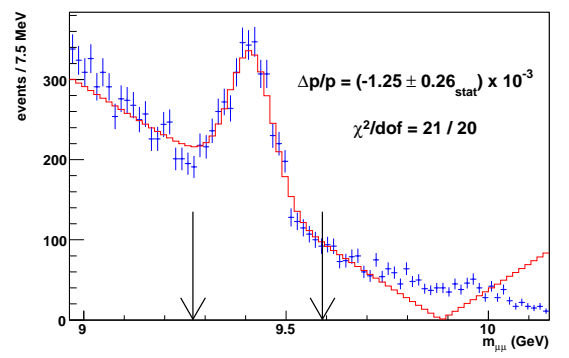

Figure F.9: Fitted $\Delta p / p$ of the ninth $\left\langle 1 / p_{T}^{\mu}\right\rangle$ bin in Figure 8.18. 


\section{Appendix G}

\section{Combining Errors}

Sometimes we are confronted with a situation where the result of a measurement is given in terms of two or more measurements. The calculation of the acceptances of $Z \rightarrow \mu \mu, W \rightarrow \tau \nu$ and $W \rightarrow \mu \nu$ falls well into this category. For $y$ as a function of $\left(x_{1}, \cdots, x_{n}\right)$ and assuming $x_{i} \mathrm{~s}(i=1, \cdots, n)$ are independent, the variance of $y\left(\sigma_{y}^{2}\right)$ can be calculated by first differentiating, then summing over the quadrature of each independent variables:

$$
\sigma_{y}^{2}=\sum_{i=1}^{n}\left(\frac{\partial y}{\partial x_{i}}\right)^{2} \sigma_{x_{i}}^{2}
$$

More specifically, for forms like $y=x_{1}^{a} x_{2}^{b}$, we have:

$$
\sigma_{y}^{2}=y^{2} \cdot\left[\left(\frac{a}{x_{1}}\right)^{2} \sigma_{x_{1}}^{2}+\left(\frac{b}{x_{2}}\right)^{2} \sigma_{x_{2}}^{2}\right]
$$

We can thus calculate the errors of acceptance fraction in Table 9.1 by using $a=1$, $b=-1$. Note the errors of acceptance (A) in percentage is obtained by assuming the number of events follow Poisson distribution, i.e., $\sigma_{x_{i}}=\sqrt{n_{i}}$. 


\section{Appendix $\mathbf{H}$}

\section{QCD Jet Background in CMX-muons}

The procedure to estimate QCD jet background using CMX muons is similar to the procedure using CMUP muons. The $\log _{10}$ distributions of trkIso and hadIso for $Z$ muons of CMX type are shown in Figure H.1. Figure H.2 compares $Z$ MC signal muon with $W$ data QCD jets, which are inputs for ROOT-JETNET. After NN training, we apply the obtained training weights to the $Z$ CMX muons from data. Three basic distributions from NN are shown in Figure H.3 a. We find that a "consistent-withzero" QCD jet background - $\left(-0.04 \pm 0.05_{\text {stat }}\right) \%$ - minimizes the $\chi^{2}$ (see Figure H.3 c). This illustrates the robustness of NN method for CMX-muon. We then apply the same trained NN to $W$ muons with the signal file replaced by $W$ CMX-muons from $W \rightarrow \mu \nu \mathrm{MC}$ samples, and the test file replaced by $W$ CMX-muons from $W$ data while the QCD background control sample fixed. We find $\left(0.06 \pm 0.02_{\text {stat }}\right) \%$ QCD background for $W$ CMX-muons. The corresponding plots are shown in Figure H.3 b and Figure H.3 d. 

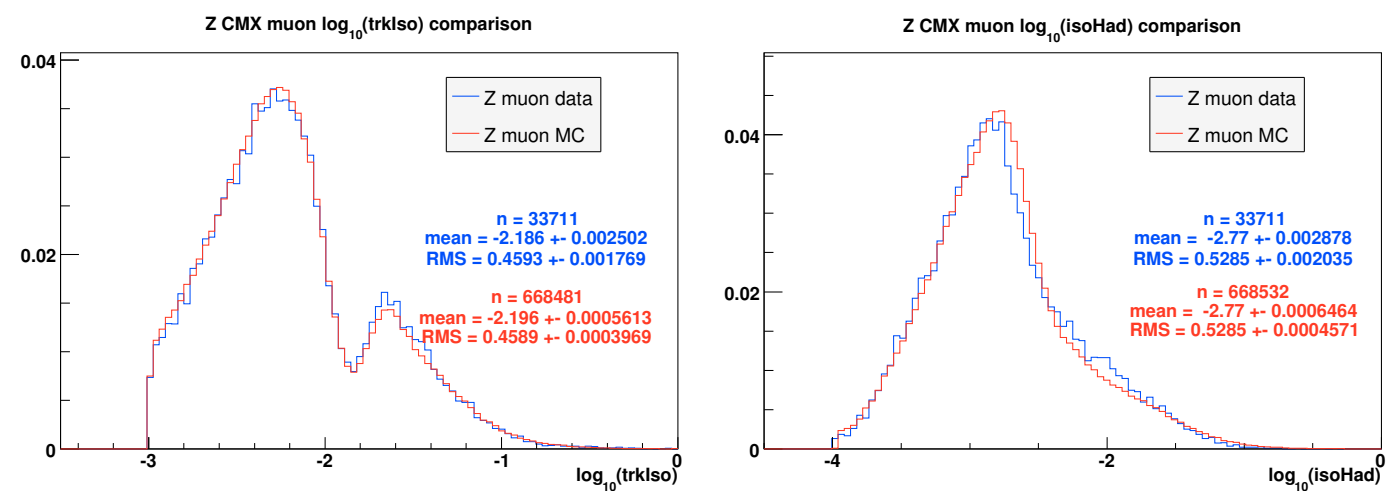

Figure H.1: The log distributions of trkIso (left) and hadIso (right) for $Z$ muons of CMX type. The red histogram is for $Z$ CMX muons from MC while the blue histogram is for $Z$ CMX muons from data.
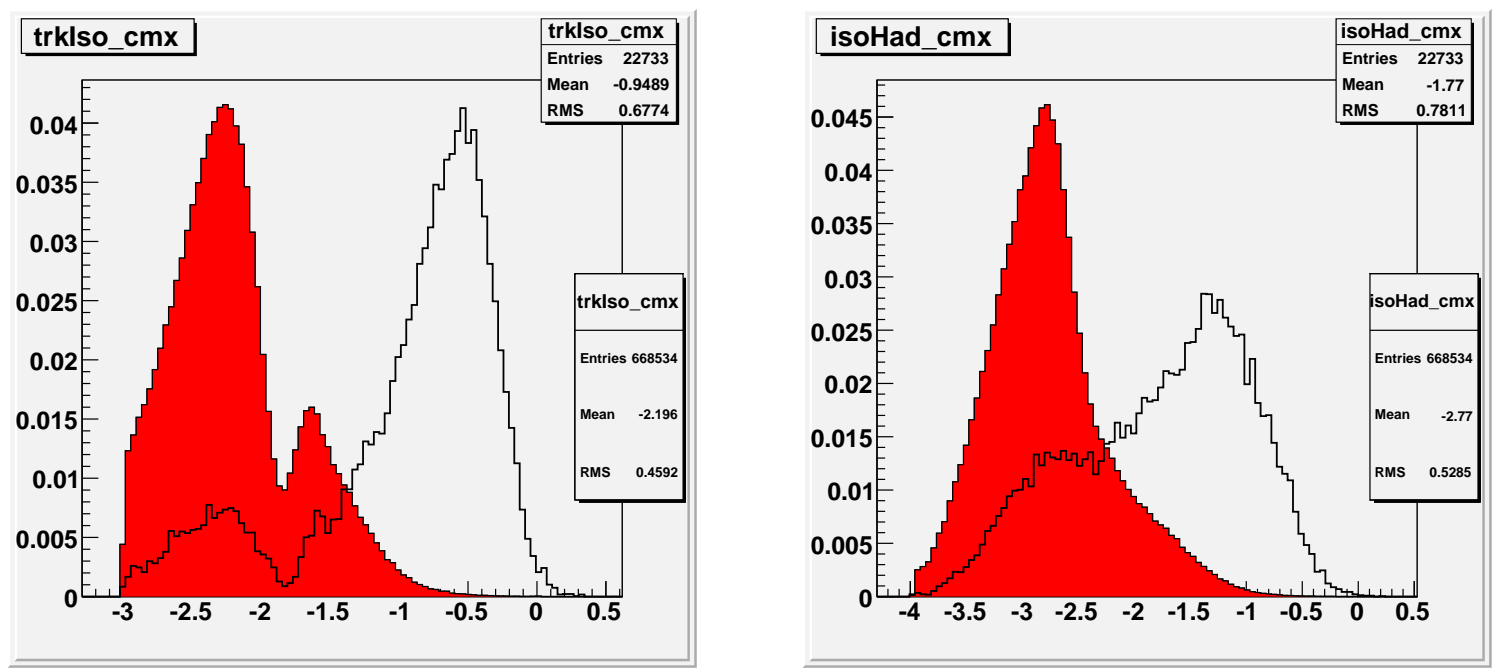

Figure H.2: The log distributions of trkIso (left) and hadIso (right) for $Z$ muons of CMX type. The red histogram is for $Z \mathrm{MC}$ muon while the black histogram is for QCD jet events from $W$ boson candidates with $p_{T}^{\nu}<10 \mathrm{GeV}$ and $u_{T}<45 \mathrm{GeV}$. 


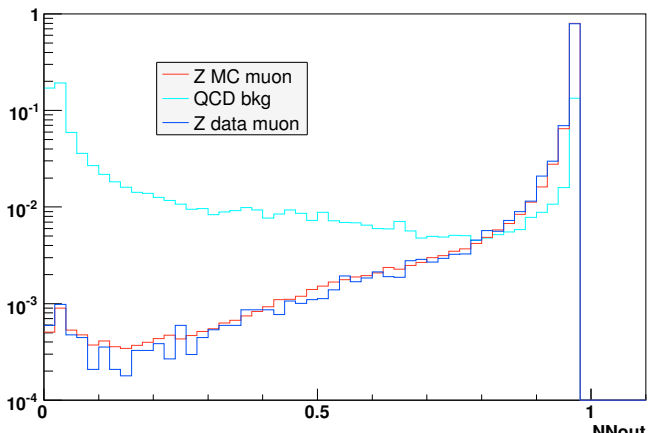

(a) Z CMX-muon NN output

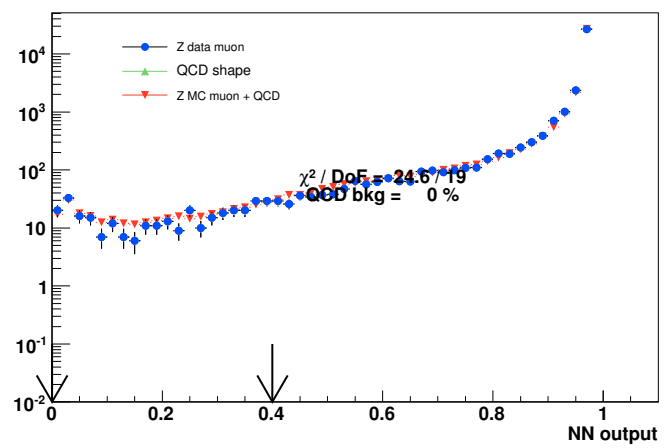

(c) Best $\chi^{2}$ fit for $Z$ CMX-muon NN out

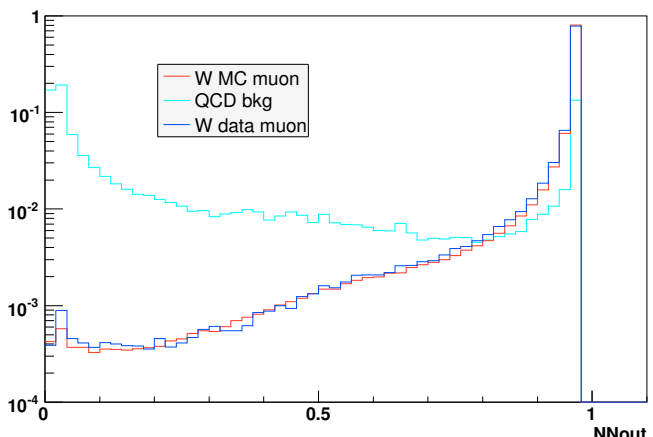

(b) $W$ CMX-muon NN output

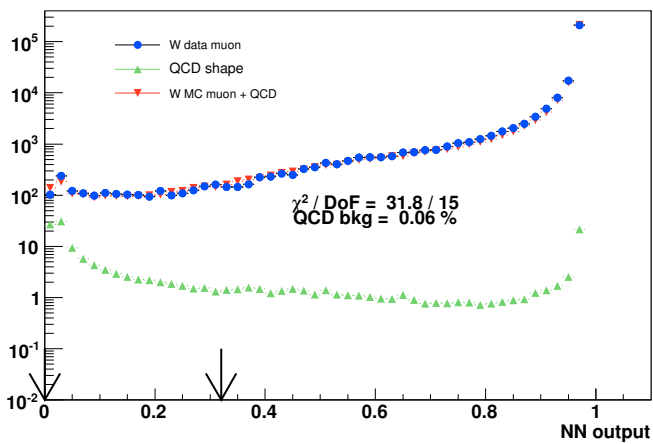

(d) Best $\chi^{2}$ fit for $W$ CMX-muon NN out

Figure H.3: The NN output distributions for $Z$ MC CMX-muons, $Z$ data CMXmuons and QCD jet events (left), and $W$ MC CMX-muons, $W$ data CMX-muons and QCD control sample (right).
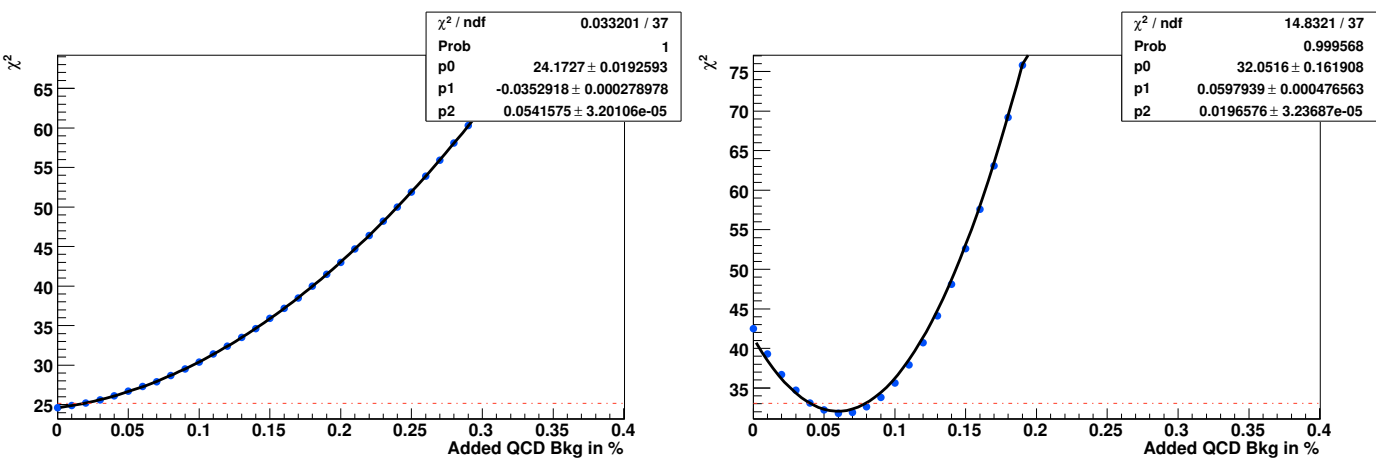

Figure H.4: $\chi^{2}$ as a function of the fraction of QCD jet background for $Z$ CMX muons (left) and $W$ CMX muons (right). 


\section{Appendix I}

\section{Effects of Fit Window}

For each chosen fit window, as illustrated in Figure 11.5, a total of 400 pseudoexperiments are simulated using PYTHIA-generated $W \rightarrow \mu \nu$ events passing through the fast detector simulation. The spread of the $m_{W}$ fit values is fitted with a Gaussian function. The extracted standard deviation $\sigma$ is treated as the statistical error associated with the chosen fit window and plotted in Figure 11.5.

Figure I.1 (Figure I.2) shows the statistical variations on $m_{W}$ fits using transverse mass $m_{T}$ distribution at different lower (upper) edges while the upper (lower) edges fixed at $90 \mathrm{GeV}(65 \mathrm{GeV}$ ). Figure I.3 (Figure I.4) shows the statistical variations on $m_{W}$ fits using muon transverse momentum $p_{T}^{\mu}$ distribution at different lower (upper) edges while the upper (lower) edges fixed at $48 \mathrm{GeV}(32 \mathrm{GeV})$. Figure I.5 (Figure I.6) shows the statistical variations on $m_{W}$ fits using missing transverse momentum $p_{T}^{\nu}$ distribution at different lower (upper) edges while the upper (lower) edges fixed at $48 \mathrm{GeV}(32 \mathrm{GeV})$. We can see the statistical variations increase as the fit window range gets bigger or smaller than the nominal values. 

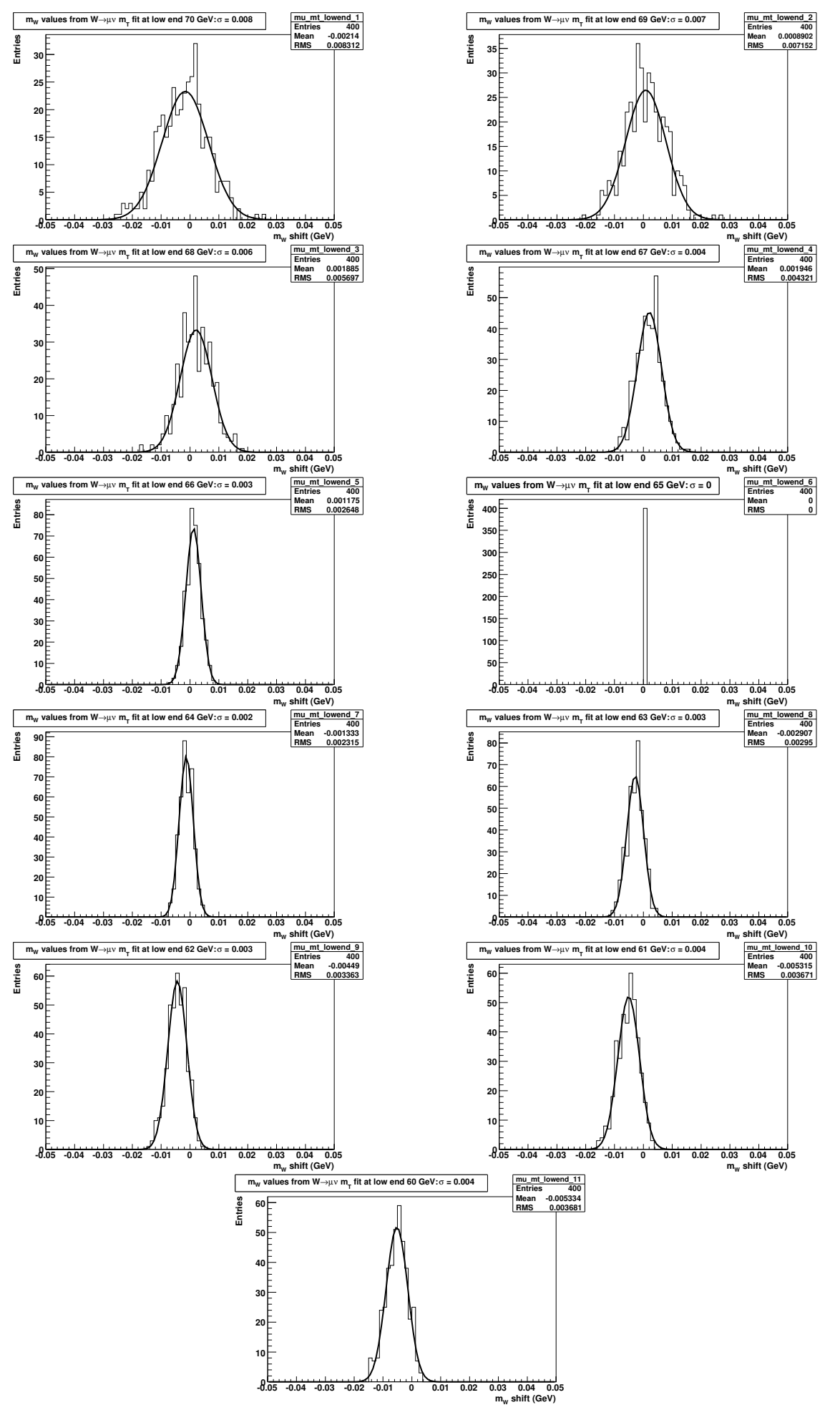

Figure I.1: The statistical variations in measured $m_{W}$, using simulated $W \rightarrow \mu \nu$ events, as the lower edge of fit range in $m_{T}$ spectrum changes from $70 \mathrm{GeV}$ to 60 $\mathrm{GeV}$ with a step size $-1 \mathrm{GeV}$ while the upper edge fixed at $90 \mathrm{GeV}$. 


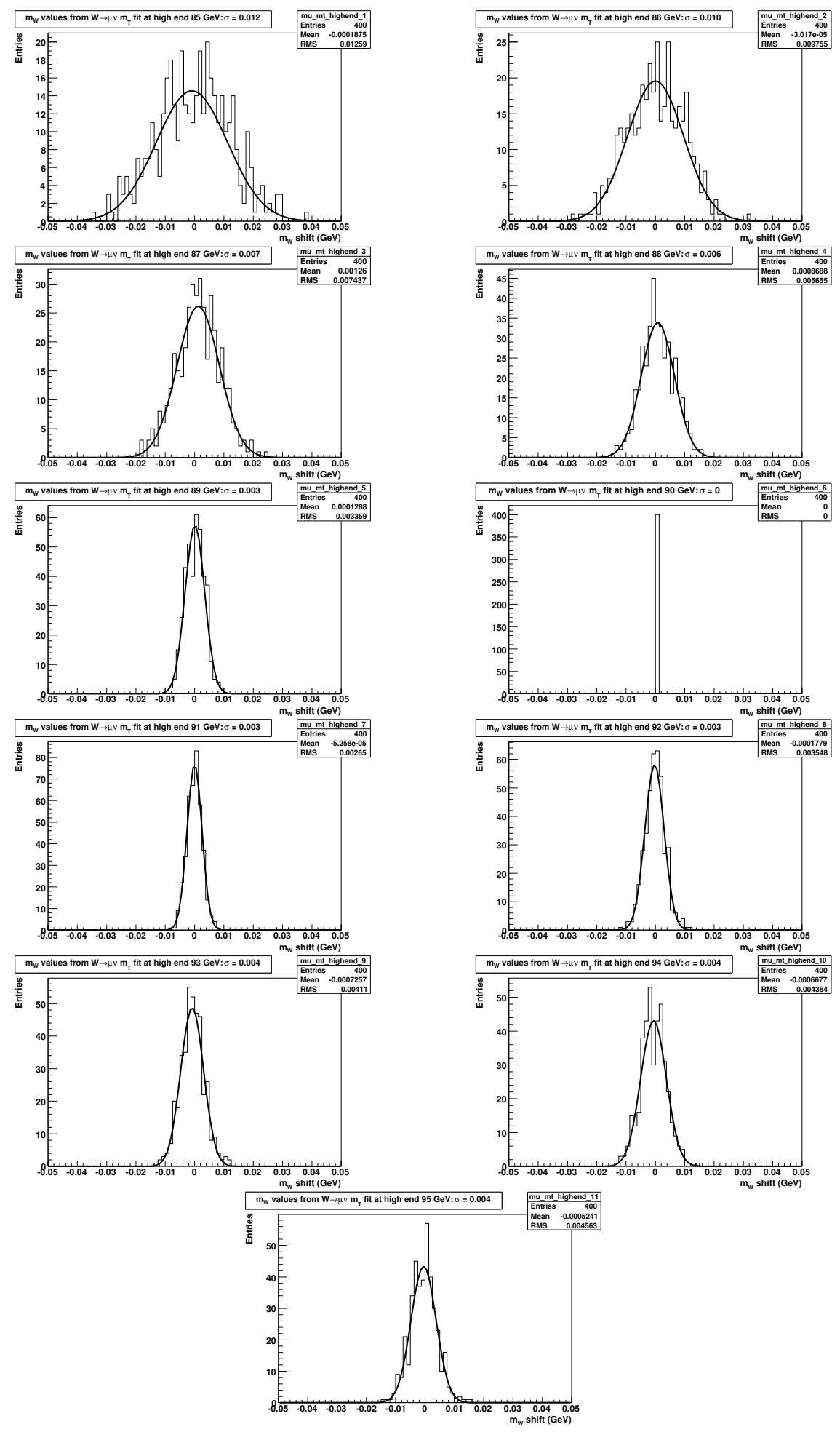

Figure I.2: The statistical variations in measured $m_{W}$, using simulated $W \rightarrow \mu \nu$ events, as the upper edge of fit range in $m_{T}$ spectrum changes from $85 \mathrm{GeV}$ to 95 $\mathrm{GeV}$ with a step size $+1 \mathrm{GeV}$ while the lower edge fixed at $65 \mathrm{GeV}$. 

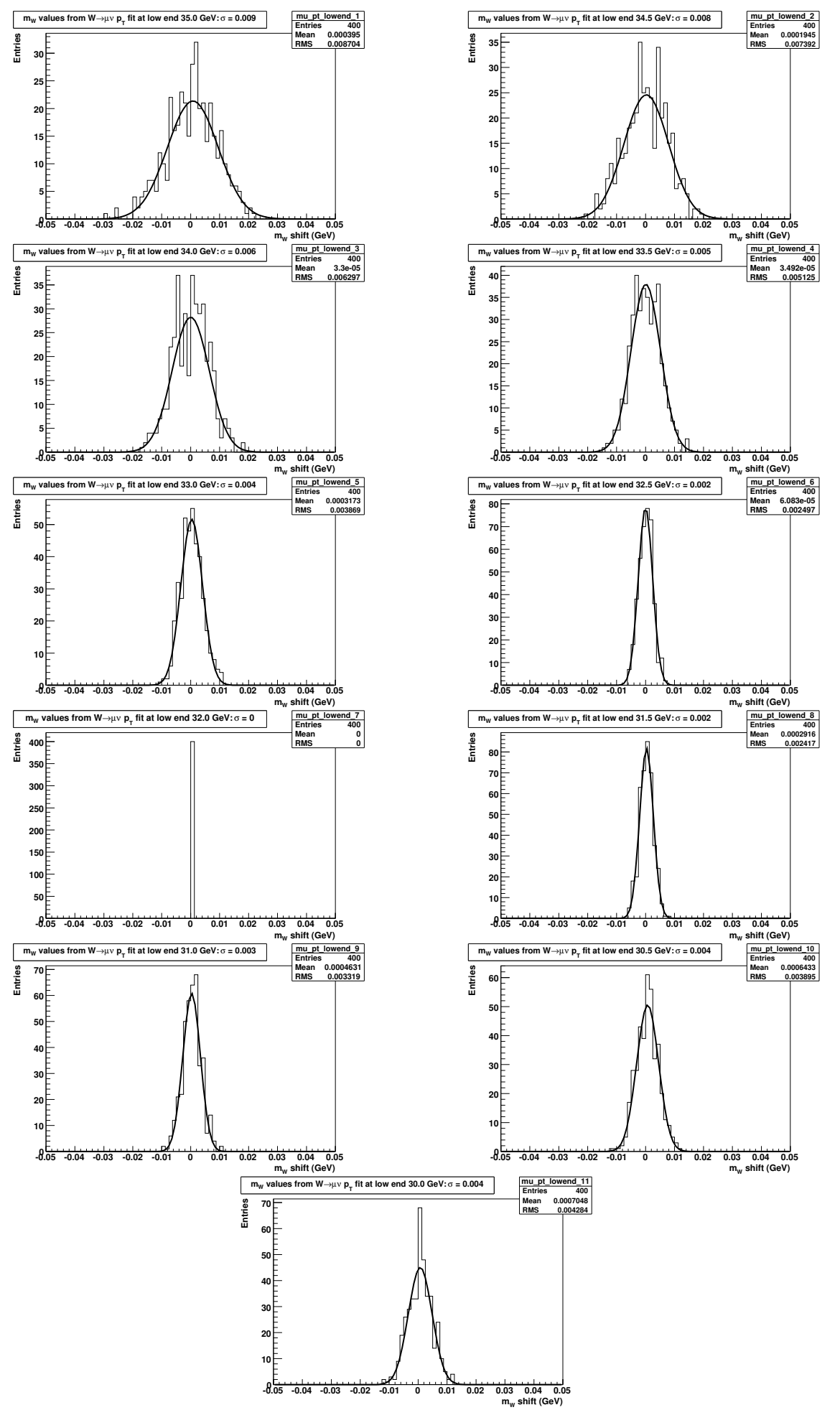

FiguRE I.3: The statistical variations in measured $m_{W}$, using simulated $W \rightarrow \mu \nu$ events, as the lower edge of fit range in $p_{T}^{\mu}$ spectrum changes from $35 \mathrm{GeV}$ to $30 \mathrm{GeV}$ with a step size $-0.5 \mathrm{GeV}$ while the upper edge fixed at $48 \mathrm{GeV}$. 

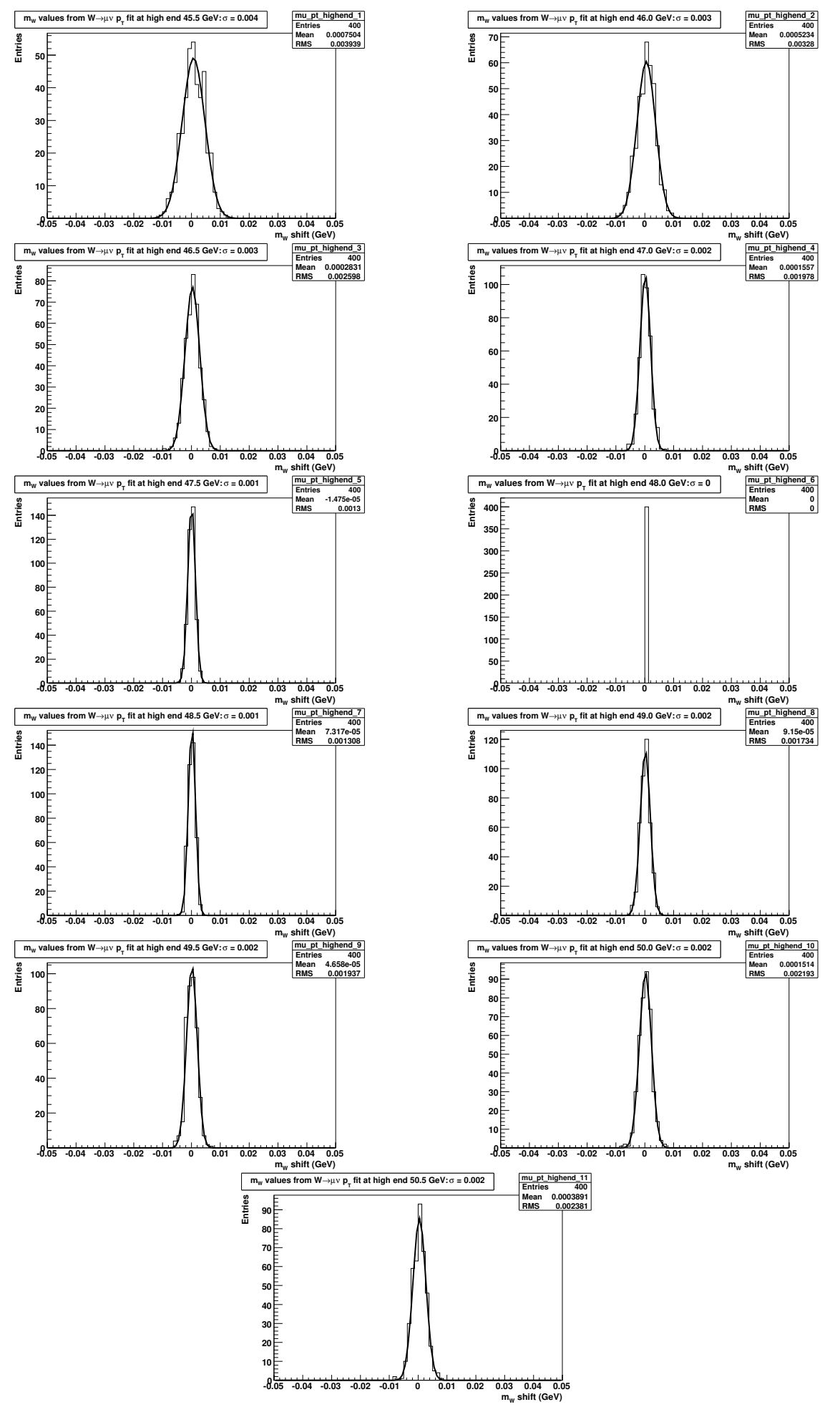

Figure I.4: The statistical variations in measured $m_{W}$, using simulated $W \rightarrow \mu \nu$ events, as the upper edge of fit range in $p_{T}^{\mu}$ spectrum changes from $45.5 \mathrm{GeV}$ to 50.5 $\mathrm{GeV}$ with a step size $+0.5 \mathrm{GeV}$ while the lower edge fixed at $32 \mathrm{GeV}$. 


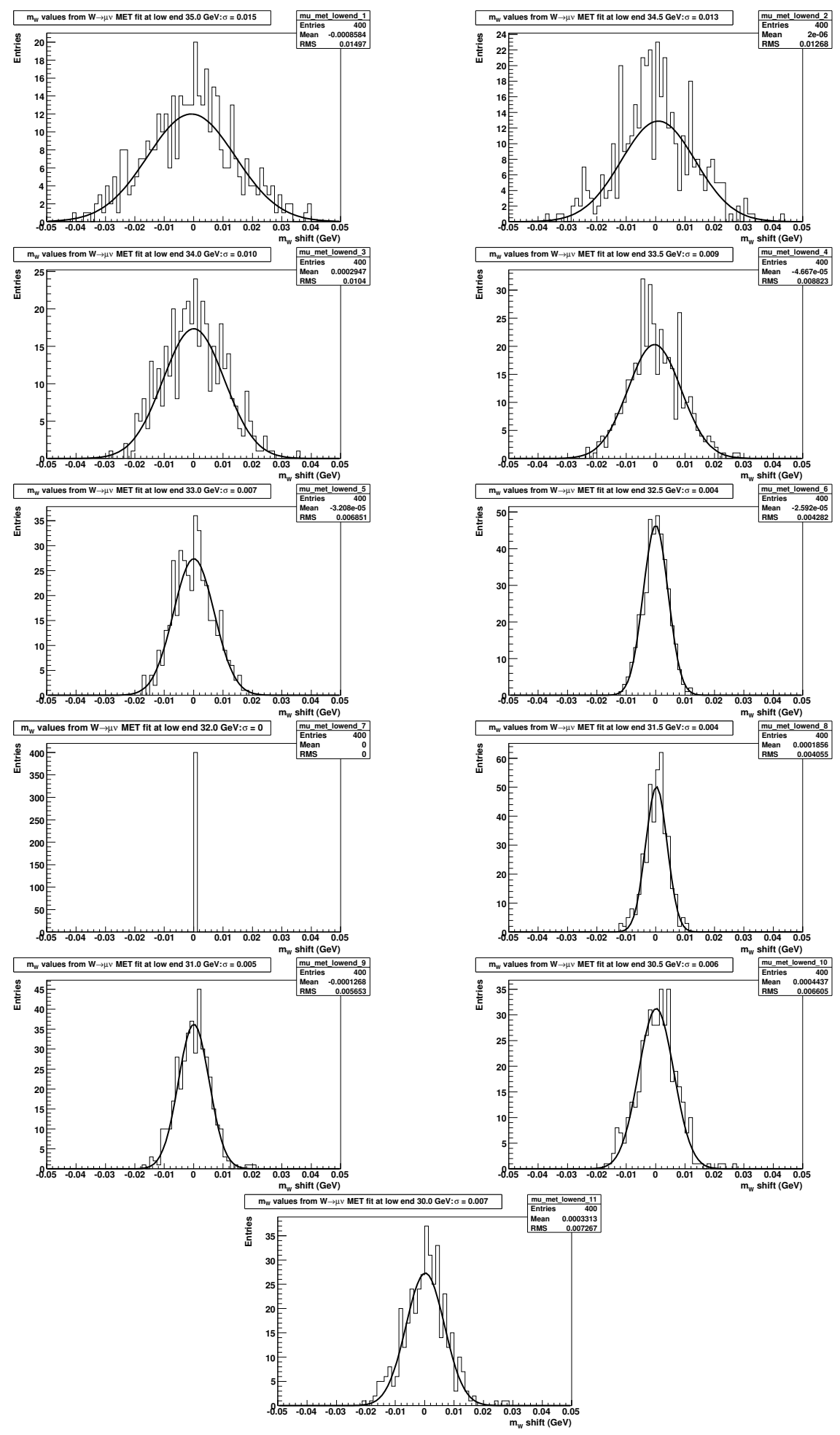

FiguRE I.5: The statistical variations in measured $m_{W}$, using simulated $W \rightarrow \mu \nu$ events, as the lower edge of fit range in $p_{T}^{\nu}\left(\not \not p_{T}\right)$ spectrum changes from $35 \mathrm{GeV}$ to $30 \mathrm{GeV}$ with a step size $-0.5 \mathrm{GeV}$ while the upper edge fixed at $48 \mathrm{GeV}$. 


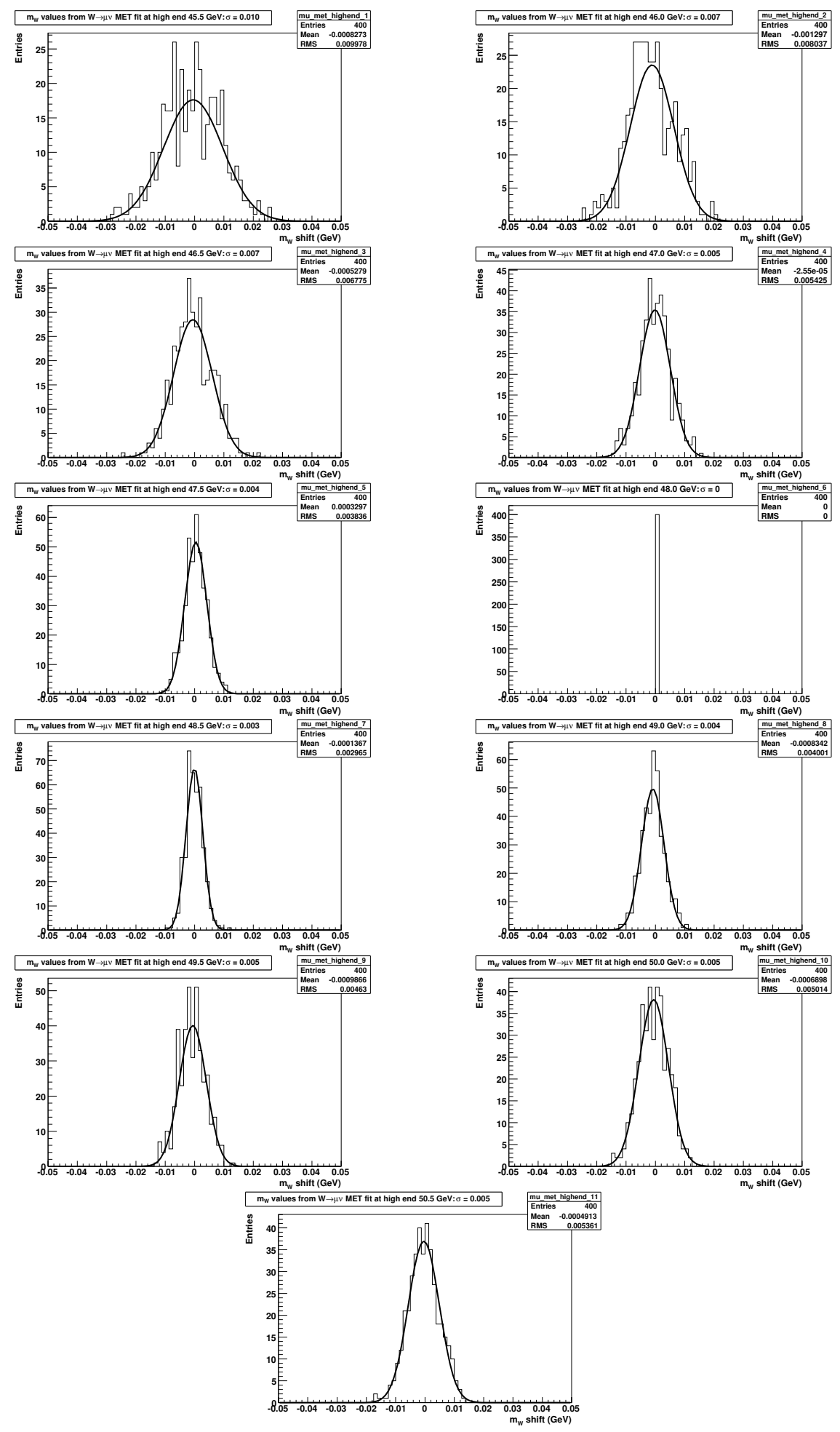

FiguRE I.6: The statistical variations in measured $m_{W}$, using simulated $W \rightarrow \mu \nu$ events, as the upper edge of fit range in $p_{T}^{\nu}\left(\not p_{T}\right)$ spectrum changes from $45.5 \mathrm{GeV}$ to $50.5 \mathrm{GeV}$ with a step size $+0.5 \mathrm{GeV}$ while the lower edge fixed at $32 \mathrm{GeV}$. 


\section{Appendix $\mathbf{J}$}

\section{$m_{T}, p_{T}^{e}$ and $p_{T}^{\nu}$ Fit Correlations in $W \rightarrow e \nu$ \\ Channel}

The same procedure is adopted in studying the correlations between $m_{W}$ mass fits in $W \rightarrow e \nu$ channel. The scatter plots are shown in Figure J.1 and results are summarized in Table J.1.

As discussed in Chapter 11, we generate 400 groups of correlated $m_{T}, p_{T}^{\mu}$ and $p_{T}^{\nu} m_{W}$ fit values in $W \rightarrow e \nu$ channel using the SVD method then evaluate the correlation coefficient $r_{i j}(i, j=1,2,3)$. We then repeat the same evaluation process 1000 times get distributions of $r_{i j}$. The standard deviation of the Gaussian fit gives an estimate of the statistical error on correlation coefficient $r_{i j}$. The results of Monte Carlo study are summarized in Figure J.2 (a), (b) and (c).

One thing to note is that we have altogether 100 million PYTHIA events and we split them equally into 400 sub-samples. Each of our $400 m_{W}$ fits using pseudoexperiments gives a statistical uncertainty of about $(48,54,66) \mathrm{MeV}$ for $m_{T}, p_{T}^{\mu}$ and $p_{T}^{\nu}$ fits (as can be seen from Figure J.1). This is about 3 times as big as the statistical uncertainty when we fit data. It thus suggests each of the 400 sub-sample 


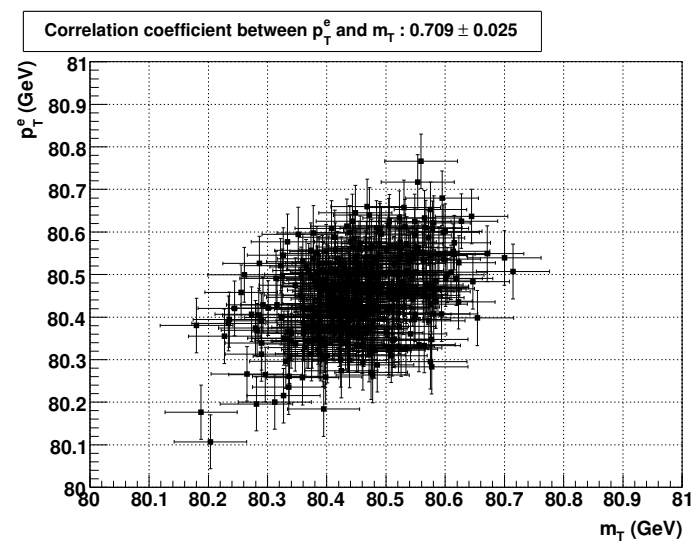

(a) Correlation between $m_{T}$ and $p_{T}^{e}$ fits.

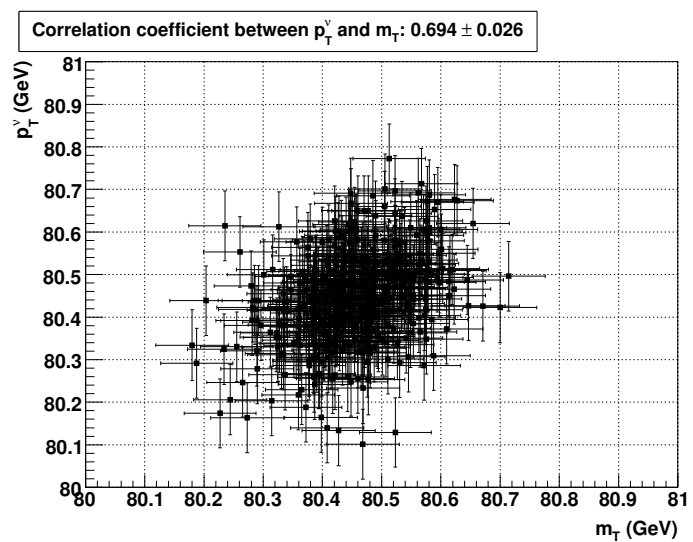

(b) Correlation between $m_{T}$ and $p_{T}^{\nu}$ fits.

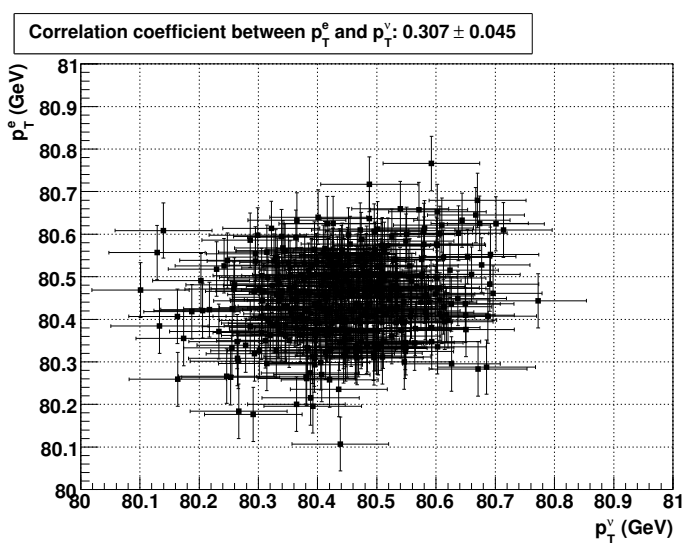

(c) Correlation between $p_{T}^{e}$ and $p_{T}^{\nu}$ fits.

Figure J.1: Correlations between $m_{T}, p_{T}^{e}$ and $p_{T}^{\nu}$ fits in $W \rightarrow e \nu$ channel.

of Pythia $W \rightarrow \mu \nu$ events is only about one-nineth of the statistics of the $W \rightarrow \mu \nu$ data. This limitation in statistics is due to the limited CPU resources we have, which makes it difficult to generate enough PYTHIA events in a timely way. This limited statistics, however, will not affect the calculation of statistcal correlation coefficients $\rho_{i j}$ among $m_{T}, p_{T}^{\mu}$ and $p_{T}^{\nu}$ fits if the number of $\left[m_{W}\left(m_{T}\right), m_{W}\left(p_{T}^{\mu}\right), m_{W}\left(p_{T}^{\nu}\right)\right]$ pairs is large and the statistical uncertainty from pseudo-experiment is signficantly smaller than the possible variation range of fitted central values. When this condition is satisfied, the shape of standard error ellipse will be insensitive to the size of statistical uncertainty from individual fit. As can be seen from Figure J.1, our settings satisfy 
Table J.1: Statistical correlations between $m_{T}, p_{T}^{e}$ and $p_{T}^{\nu}$ fits in $W \rightarrow e \nu$ channel.

\begin{tabular}{lc}
\hline \hline$W \rightarrow e \nu$ fit variable & Correlation Coefficient \\
\hline$m_{T}$ vs. $p_{T}^{e}$ & $0.709 \pm 0.025$ \\
$m_{T}$ vs. $p_{T}^{\nu}$ & $0.694 \pm 0.026$ \\
$p_{T}^{e}$ vs. $p_{T}^{\nu}$ & $0.307 \pm 0.045$ \\
\hline \hline
\end{tabular}

this condition. We have cross-checked the estimation of $\rho_{i j}$ by adjusting the number of pseduo-experiments and the number of MC events for each pseudo-experiment while keeping the total number of the MC events fixed at 100 million. As long as the mentioned condition is met, the change in $\rho_{i j}$ estimation is within its statistical uncertainty. So our setting will not affect correlation coefficient estimation even when we are limited by PYTHIA statistics. 


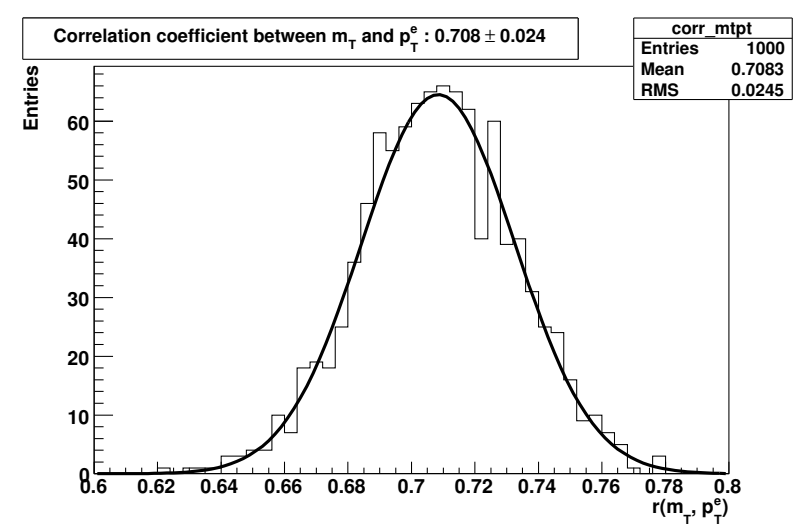

(a) Error on $m_{T}$-and- $p_{T}^{\mu}$ correlation coefficient.

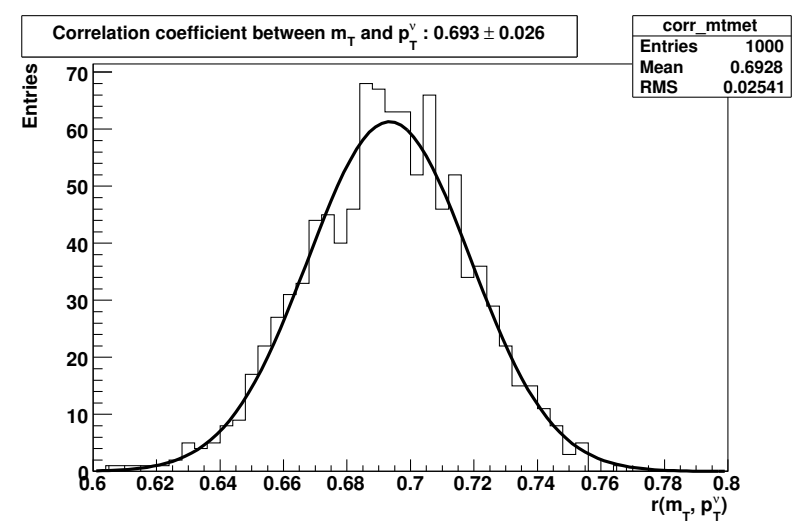

(b) Error on $m_{T}$-and- $p_{T}^{\nu}$ correlation coefficient.

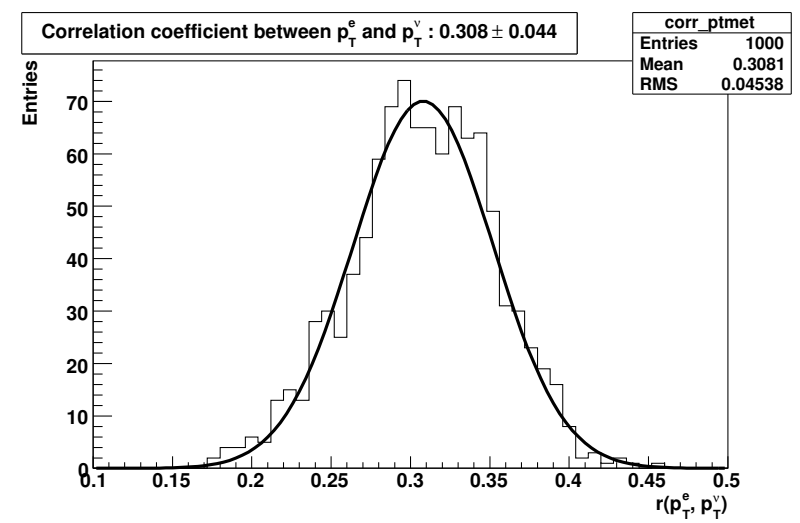

(c) Error on $p_{T}^{\mu}$-and- $p_{T}^{\nu}$ correlation coefficient.

Figure J.2: Monte Carlo study of the errors on correlation coefficients between $m_{T}$, $p_{T}^{\mu}$ and $p_{T}^{\nu}$ fits in $W \rightarrow \mu \nu$ channel. 


\section{Appendix K}

\section{Using Least Squares to Combine Measurements}

A number of measurements of the same quantity is often linearly combined by using Least Squares (LS) method, which shares the basic idea of the Best Linear Unbiased Estimate (BLUE) method. Given the covariance matrix $V_{n \times n}$, the definition of $\chi^{2}$ takes the general form:

$$
\chi^{2}(\theta)=\sum_{i, j=1}^{n}\left(y_{i}-\theta\right)\left(V^{-1}\right)_{i j}\left(y_{i}-\theta\right)
$$

The LS estimator $\hat{\theta}$ for $\theta$ can be obtained by taking the first derivative of $\chi^{2}(\theta)$ with respect to $\theta$ and setting it to zero. This leads to

$$
\hat{\theta}=\sum_{i=1}^{n} \omega_{i} \cdot y_{i}
$$

where the weights $\omega_{i}$ are given by

$$
\omega_{i}=\frac{\sum_{j=1}^{n}\left(V^{-1}\right)_{i j}}{\sum_{k, l=1}^{n}\left(V^{-1}\right)_{k l}},
$$


which satisfy

$$
\sum_{i=1}^{n} \omega_{i}=\frac{\sum_{i, j=1}^{n}\left(V^{-1}\right)_{i j}}{\sum_{k, l=1}^{n}\left(V^{-1}\right)_{k l}}=1
$$

One thing we need to point out is that, when the individual measurements $y_{i}(i=$ $1, \cdots, n)$ are all unbiased estimates of true value $\theta, \hat{\theta}$ is also unbiased estimate of true value $\theta$ :

$$
E[\hat{\theta}]=\sum_{i=1}^{n} \omega_{i} \cdot E\left[y_{i}\right]=\theta \sum_{i=1}^{n} \omega_{i}=\theta
$$

According to the Gauss-Markov theorem [92], the weights described by Eqn. (K.3) lead to the unbiased estimator $\hat{\theta}$ with the smallest possible variance given by

$$
\sigma^{2}(\hat{\theta})=\sum_{i, j=1}^{n} \omega_{i} V_{i j} \omega_{j}=\mathbf{\Omega}^{\mathbf{T}} \mathbf{V} \boldsymbol{\Omega}
$$

where $\Omega$ is a column vector with weights $\omega_{i}(i=1, \cdots, n)$ and $\boldsymbol{\Omega}^{\mathbf{T}}$ is the transpose of $\Omega$.

\section{K.0.1 Uncorrelated measurements}

In the special case of uncorrelated measurements $y_{i}$, we have $\left(V^{-1}\right)_{i j}=\delta_{i j} / \sigma_{i}^{2}$ and Eqns. (K.2) (K.3) can be simplified as

$$
\hat{\theta}=\frac{\sum_{i=1}^{n} y_{i} / \sigma_{i}^{2}}{\sum_{j=1}^{n} 1 / \sigma_{j}^{2}}
$$

The variance of $\hat{\theta}$ is

$$
\sigma^{2}=\frac{1}{\sum_{i=1}^{n}\left(1 / \sigma_{i}^{2}\right)} .
$$




\section{K.0.2 Two measurements with correlation}

Suppose we have first (second) measurement of $x$ to be $x_{1(2)} \pm \sigma_{1(2)}$ and the two measurements have common uncertainty $\sigma^{\text {corr }}$, to combine them we can first compute the uncorrelated part of the uncertainty.

$$
\begin{aligned}
& \left(\sigma_{1}^{\text {uncorr }}\right)^{2}=\sigma_{1}^{2}-\left(\sigma^{\text {corr }}\right)^{2} \\
& \left(\sigma_{2}^{\text {uncorr }}\right)^{2}=\sigma_{2}^{2}-\left(\sigma^{\text {corr }}\right)^{2}
\end{aligned}
$$

The combined central value from $x_{1}$ and $x_{2}$ is:

$$
E(x)=\frac{x_{1}\left(\sigma_{2}^{\text {uncorr }}\right)^{2}+x_{2}\left(\sigma_{1}^{\text {uncorr }}\right)^{2}}{\left(\sigma_{1}^{\text {uncorr }}\right)^{2}+\left(\sigma_{2}^{\text {uncorr }}\right)^{2}}
$$

The combined variance from measurement 1 and 2 after taking the correlation into account is:

$$
\sigma^{2}=\frac{\sigma_{1}^{2} \sigma_{2}^{2}-\left(\sigma^{\text {corr }}\right)^{4}}{\sigma_{1}^{2}+\sigma_{2}^{2}-2\left(\sigma^{\text {corr }}\right)^{2}}
$$

We can use the above formula to derive the combined momentum scale when the $Z$ mass fit result relative to world-average value gets averaged in.

Eqn. (8.17) from $Z$ mass fit gives $\left(-135 \pm 14_{\text {total }}\right) \times 10^{-5}$ and Eqn. (8.12) from combined $J / \psi$ and $\Upsilon$ fits gives $\left(-126 \pm 10_{\text {total }}\right) \times 10^{-5}$ on $\Delta p / p$, respectively. The systematic uncertainties due to QED $\left(5 \times 10^{-5}\right)$ and alignment $\left(2 \times 10^{-5}\right)$ are common to both low mass $J / \psi, \Upsilon$ and high mass $Z$. We thus have $\sigma_{1}=10, \sigma_{2}=14$ and $\sigma^{\text {corr }}=\sqrt{5_{\mathrm{QED}}^{2}+2_{\text {align }}^{2}}=5.4$. Plug in these numbers in Eqn. (K.11) and Eqn. (K.12) we have:

$$
(\Delta p / p)=(-128.68 \pm 8.88) \times 10^{-5} \approx(-129 \pm 9) \times 10^{-5}
$$

From this we can get the uncorrelated uncertainty between $Z$ mass fit result and combined $J / \psi$ and $\Upsilon$ result to be $\sqrt{9^{2}-5_{\text {QED }}^{2}-2_{\text {align }}^{2}} \approx 7 \mathrm{MeV}$. Thus the final 
momentum scale $\Delta p / p$ from $J / \psi, \Upsilon$ and $Z$ is

$$
(\Delta p / p)_{\text {final }}=\left(-129 \pm 7_{\text {uncorr }} \pm 2_{\text {align }} \pm 5_{\mathrm{QED}}\right) \times 10^{-5} .
$$

This is exactly what we have shown as Eqn. (8.18) in Chapter 8.

Another application of Eqn. (K.12) is the combination of the systematic uncertainties on $m_{W}$ due to $p_{T}(W)$-related parameters $g_{2}$ and $\alpha_{s}$. Using the correlation coefficient -0.7 between $g_{2}$ and $\alpha_{s}$, and the corresponding uncertainties shown in Section 6.2 , we get the combined systematic uncertainty on $m_{W}$ to be $3 \mathrm{MeV}, 9$ $\mathrm{MeV}$ and $4 \mathrm{MeV}$ for $m_{T}, p_{T}^{\mu}$ and $p_{T}^{\nu}$ fits.

\section{K.0.3 Combine statistical uncertainties from $m_{T}, p_{T}^{\mu}$ and $p_{T}^{\nu}$ fits}

We illustrate in this section the combination of statistical uncertainties from $m_{T}$, $p_{T}^{\mu}$ and $p_{T}^{\nu}$ fits. As have been shown in Section 11.6, the statistical correlations between $m_{T}$ and $p_{T}^{\mu}, m_{T}$ and $p_{T}^{\nu}, p_{T}^{\mu}$ and $p_{T}^{\nu}$ have been measured to be $(67.2 \pm 2.8) \%$, $(65.8 \pm 2.8) \%,(25.5 \pm 4.7) \%$, respectively. Using the statistical uncertainy of $m_{T}, p_{T}^{\mu}$ and $p_{T}^{\nu}$ fits $(16 \mathrm{MeV}, 18 \mathrm{MeV}$ and $22 \mathrm{MeV})$, we can construct the covariance matrix as:

$$
\mathbf{V}=\left(\begin{array}{ccc}
\sigma_{1}^{2} & \rho_{12} \sigma_{1} \sigma_{2} & \rho_{13} \sigma_{1} \sigma_{2} \\
\rho_{12} \sigma_{1} \sigma_{2} & \sigma_{2}^{2} & \rho_{23} \sigma_{2} \sigma_{3} \\
\rho_{13} \sigma_{1} \sigma_{3} & \rho_{23} \sigma_{2} \sigma_{3} & \sigma_{3}^{2}
\end{array}\right)=\left(\begin{array}{ccc}
256 & 194 & 232 \\
194 & 324 & 101 \\
232 & 101 & 484
\end{array}\right)
$$

Its matrix inverse can be calculated by using LU decomposition [94]:

$$
\mathbf{V}^{-1}=\left(\begin{array}{ccc}
0.013365 & -0.006423 & -0.005066 \\
-0.006423 & 0.006388 & 0.001746 \\
-0.005066 & 0.001746 & 0.004130
\end{array}\right)
$$

Applying Eqn. (K.3), we get $\omega_{1}=0.427, \omega_{2}=0.389$ and $\omega_{3}=0.184$. From these weights and using Eqn. (K.6), we can calculate the combined statistical uncertainty to be $\sigma_{\text {stat }}^{\text {combined }}=15.1 \mathrm{MeV} \approx 15 \mathrm{MeV}$. 
We vary the correlation coefficients by their corresponding statistical uncertainties to evaluate the change in combined statistical uncertainty from $m_{T}, p_{T}^{\mu}$ and $p_{T}^{\nu}$ fits. There are altogether $3 \times 3 \times 3=27$ combinations of the correlation coefficients and thus 27 statistical covaraince matrix. For each covariance matrix, we use LU decomposition to find its matrix inverse and then use Eqn. (K.3) and Eqn. (K.6) to calculate the corresponding combined statistical uncertainty.

The distribution of combined statistical uncertainty is shown in Figure K.1. The uncertainty on the combined statistical uncertainty is found to be $0.1 \mathrm{MeV}$ and is thus negligible.

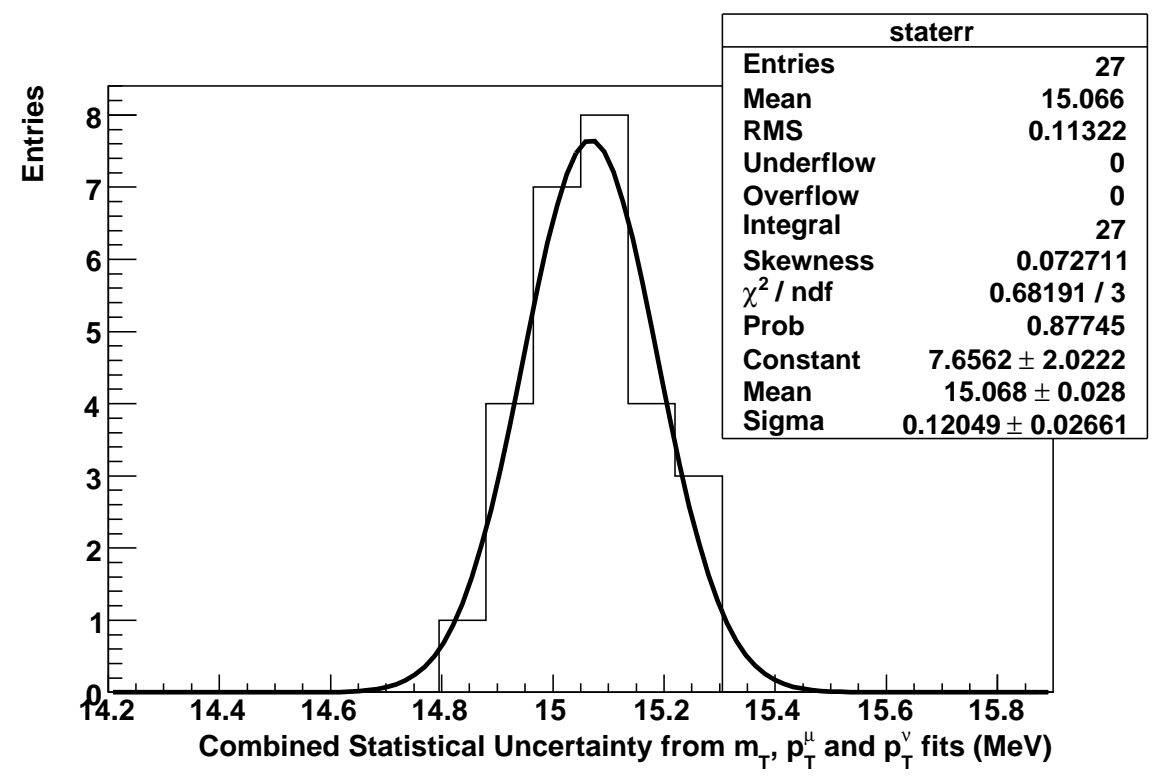

FiguRE K.1: Variation of combined statistical uncertainty from $m_{T}, p_{T}^{\mu}$ and $p_{T}^{\nu}$ fits due to the $1 \sigma$ statistical uncertainty of correlation coefficients. 


\section{Bibliography}

[1] D. Griffiths, Introduction to Elementary Particles. Wiley, John \& Sons (1987).

[2] D. Perkins, Introduction to High Energy Phyiscs, 4th edition, Addison-Wisley (2000).

[3] G. Altarelli, Status of the Standard Model and Beyond, 38th Rencontres de Moriond, hep-ph/030605 (2003).

[4] J. Goldstone, A. Salam and S. Weinberg, Broken Symmetries, Physical Review 127:154-164 (1962).

[5] A. Goshaw, Physics 346 Lecture Notes, Duke University (2007).

[6] V. Barger and R. Phillips, Collider Physics (updated edition), Westview Press (1996).

[7] G. Abbiendi et al., ALEPH Collaboration, Phys. Lett. B:565 (2003).

[8] T. Aaltonen et al., CDF Collaboration, First Run II Measurement of the $W$ Boson Mass, Phys. Rev. D 77 (2008).

[9] S. Jindariani et al., Luminosity Uncertainty for Run 2 up until August 2004, CDF Note $\mathbf{7 4 4 6}$ (2005).

[10] S. Klimenko, J. Konigsberg and T. Liss, Averaging of the Inelastic Cross Sections Measured by the CDF and the E811 Experiments, Fermilab-FN-0741 (2004).

[11] T. Aaltonen et al., CDF Collaboration, Phys. Rev. Lett 99:151801 (2007).

[12] V. M. Abazov et al., DØ Collaboration, Phys. Rev. Lett 103:141801 (2009).

[13] LEP Electroweak Working Group, http://lepewwg. web.cern.ch/LEPEWWG. 
[14] S. Dawson, http://quark.phy.bnl.gov/ dawson/tasi3.pdf, talk given at First CERN-Fermilab Hadron Collider Physics Summer School, Fermilab (2006).

[15] M. Awramik et al., Phys. Rev. D 69:053006 (2004).

[16] The Tevatron Electroweak Working Group, arXiv:1109.2163 (2011).

[17] S. Heinemeyer et al., J. High Energy Phys. 08:052 (2006).

[18] G. Arnison et al., UA1 Collaboration, Phys. Lett. B 122:103 (1983).

[19] M. Banner et al., UA2 Collaboration, Phys. Lett. B 122:476 (1983).

[20] J. Alitti et al., UA2 Collaboration, Phys. Lett. B 241:150 (1990).

[21] J. Abdallah et al. (DELPHI Collaboration), Eur. Phys. J. C 55:1 (2008).

[22] P. Achard et al. (L3 Collaboration), Eur. Phys. J. C 45:569 (2006).

[23] G. Abbiendi et al. (OPAL Collaboration), Eur. Phys. J. C 45:307 (2006).

[24] S. Schael et al. (ALEPH Collaboration), Eur. Phys. J. C 47:309 (2006).

[25] J. Alcarez et al. (LEP Collaboration), arXiv:hep-ex/0612034 (2006).

[26] T. Affolder et al. (CDF Collaboration), Phys. Rev. D 64:052001 (2001).

[27] V. M. Abazov et al. (DØ Collaboration), Phys. Rev. D 66:012001 (2002); B. Abbott et al. (DØ Collaboration), Phys. Rev. D 58:092003 (1998); B. Abbott et al. (DØ Collaboration), Phys. Rev. D 62:092006 (2000).

[28] V. M. Abazov et al., Phys. Rev. D 70:092008 (2004).

[29] T. Aaltonen et al. (CDF Collaboration), Phys. Rev. D 77:112001 (2008).

[30] V. M. Abazov et al. (DØ Collaboration), Phys. Rev. Lett 103:141801 (2009).

[31] The Tevatron Electroweak Working Group, arXiv:0908.1374 (2009)

[32] Particle Data Group, Review of Particle Physics, J. Phys. G 37:075021 (2010)

[33] Tevatron Rookie Book, http://www-bdnew.fnal.gov/operations/rookie books/Tevatron_v2.3.pdf 
[34] S. Mishra, High Luminosity Operation of the Fermilab Accelerator Complex, FERMILAB-CONF-03-194 (2003).

[35] T. Affolder et al., CDF Central Outer Tracker, Nucl. Instrum. Meth. A 526:249 (2004).

[36] C. Hill et al., L00: Operation Experience and Performance of the CDF II Silicon Detector, Nucl. Instrum. Meth. A 530:1 (2004).

[37] A. Sill et al., SVX-II: CDF Run II Silicon Tracking Projects, Nucl. Instrum. Meth. A 447:1 (2000).

[38] A. Affolder et al., ISL: Intermediate Silicon Layers Detector for the CDF Experiment, Nucl. Instrum. Meth. A 453:84 (2000).

[39] O. Sterlzer-Chilton, Ph.D. thesis, First Measurement of the $W$ Boson Mass with CDF in Run 2, Physics Department, University of Toronto (2006).

[40] GEANT4, A Toolkit for the Simulation of the Passage of Particle through Matter, http://geant4 org.

[41] S. Kuhlmann et al., The CDF Calorimetry Upgrade for Run IIb, Nucl. Instrum. Meth. A 518:39 (2004).

[42] A. Artikov et al., Design and Construction of New Central and Forward Muon Counters for CDF II, Nucl. Instrum. Meth. A 538:358 (2005).

[43] S. Klimenko, Uncertainties of Luminosity measurement at the Tevatron, http: //cepa.fnal.gov/psm/MCTuning/april/klimenko.pdf. CDF Collaboration, Phys. Rev. D 50:5550 (1994).

[44] CDF DAQ and Trigger Overview, CDF Ace Training Lectures (2010).

[45] R. Thurman-Keup, A. Kotwal, M. Tecchio, and A. Byon-Wagner, $W$ Boson Physics at Hadron Colliders, Rev. Mod. Phys. 73, No. 2 (2001).

[46] Ian Vollrath, Ph.D. thesis, Measurement of the $W$ Boson Mass at the Collider Detector at Fermilab from a Fit to the Transverse Momentum Spectrum of the Muon, Department of Physics, University of Toronto (2007).

[47] Harald Cramer, Mathematical Methods of Statistics, Princeton University Press (1946). 
[48] Yu Zeng, M.S. thesis, Momentum Scale Estimation Using Maximum Likelihood Template Fitting, Department of Statistics, Duke University (2010).

[49] Yimei Huang et al., Inside-Out Tracking, CDF Note 6707 (2003).

[50] http://cdfcodebrowser.fnal.gov/CdfCode/source/include/ TrackingObjects/Tracks/CdfTrack.hh\#480

[51] CTEQ Collaboration, http://www.phys.psu.edu/ cteq/

[52] MRST Collaboration, http://durpdg.dur.ac.uk/hepdata/mrs.html

[53] Daniel Beecher, PDF systematic for Run-II W Mass, CDF note 10117 (2010).

[54] J. Pumplin et al., New Generation of Parton Distributions with Uncertainties from Global QCD Analysis, J. High Energy Phys. 0207:012 (2002).

[55] J. C. Collins, D. Soper, and G. Sterman, Nucl. Phys. B 250, 199 (1985).

[56] F. Landry, R. Brock, P. M. Nadolsky and C.-P. Yuan, Phys. Rev. D 67, 073016 (2003).

[57] G. A. Ladinsky and C.-P. Yuan, Phys. Rev. D 50:4239 (1994).

[58] D. Beecher, PDF and QCD Effects in the Precision Measurement of the $W$ boson mass at CDF, Ph.D Thesis, University of College London, CDF Note 10411 (2011).

[59] I. Vollrath, Measurement of the $W$ Boson Mass at the Collider Detector at Fermilab from a Fit to the Transverse Momentum Spectrum of the Muon, Ph.D Thesis, University of Toronto (2007).

[60] U. Baur, S. Keller and D. Wackeroth, Electroweak radiative corrections to $W$ boson production in hadronic collisions, Phys. Rev. D 59, 013002 (1998).

[61] C.M. Carloni Calame, G. Montagna, O. Nicrosini, and M. Treccani, Phys. Rev. D 69, 037301 (2004).

[62] G. Nanava, Z. Was, Eur. Phys. J. C 51:569-583 (2007).

[63] G. Nanava, Z. Was and Q. Xu, arXiv:0906.4052v2 (2010). 
[64] C.M. Carloni Calame, G. Montagna, O. Nicrosini and A. Vicini, Precision electroweak calculation of the charged current Drell-Yan process, JHEP 0612 (2006).

[65] C.M. Carloni Calame, G. Montagna, O. Nicrosini and A. Vicini, Precision electroweak calculation of the production of a high transverse-momentum lepton pair at hadron colliders, JHEP 0710:109 (2007)

[66] I. Bizjak, B. Jayatilaka and A. Kotwal, Comparison of HorACE and PHotos algorithms for photon showering, CDF note 10482 (2011).

[67] I. Bizjak, Electroweak effects in the measurement of the $W$ boson mass, CDF note 9987 (2009).

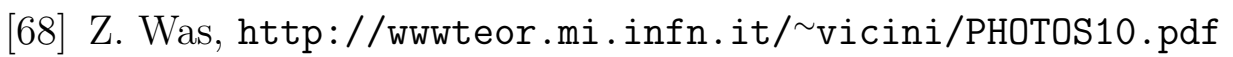

[69] Ashutosh Kotwal et al., Fast Detector Simulation for the W Mass Measurement, CDF note 7573 (2006).

[70] R. M. Sternheimer, Phys. Rev., 88:851 (1952).

[71] D. Attwood et al., The Scattering of Muons in Low $Z$ Materials, Nucl. Instrum. Meth. B 251:41-55 (2006).

[72] R. L. Gluckstern, Uncertainties in Track Momentum and Direction due to Multiple Scattering and Measurement Errors, Nucl. Instrum. Meth. A 24:381 (1963).

[73] A. Abulencia et al., CDF Collaboration, J. Phys. G: Nucl. Part. Phys. 34:2457 (2007)

[74] D. Acosta et al., CDF Collaboration, Phys. Rev. D 71, 032001 (2005)

[75] A. V. Kotwal, H. K. Gerberich, and C. Hays, Nucl. Instrum. Meth. A 506:110 (2003).

[76] T. Sjostrand, S. Mrenna and P. Skands, PYTHIA 6.4 Physics and Manual, JHEP 05 2006. The Pythia Program. http://www.thep.lu.se/ torbjorn/Pythia. html.

[77] T. Sjostrand, Comput. Phys. Commun. 82:74 (1994); R. Kleiss et al., CERN 89-08, Vol. 3 (1989) (unpublished). 
[78] P. Sutton, A. Martin, R. Roberts, and W. Stirling, Phys. Rev. D 45:2349 (1992); R. Rijen and W. van Neerven, Phys. Rev. D 51:44 (1995); R. Harlander and W. Kilgore, Phys. Rev. Lett 88:201801 (2002).

[79] D. Acosta et al. (CDF Collaboration), Phys. Rev. Lett. 94:091803 (2005).

[80] C. Hays et al., Measurement of the $W$ Boson Mass in Run 2, CDF Note $\mathbf{7 1 0 4}$ (2006).

[81] A. Abulencia et al. (CDF Collaboration), J. Phys. G 34:2457, (2007);

[82] Y. Zeng, B. Jayatilaka, A. Kotwal, C. Hays and O. Stelzer-Chilton, Muon Channel Backgrounds to the $W$ Boson Mass Measurement using $2.3 \mathrm{fb}^{-1}$ of Run 2 Data, CDF Note 10655 (2011).

[83] C. Ciobanu et al., A ROOT Interface to JETNET, CDF note 5434 (2002).

[84] L. Lonnblad et al., Comput. Phys. Commun. 81:185 (1994).

[85] C. Hays, A. Kotwal, Y. Li and O. Stelzer-Chilton, A Search for Narrow Resonances Decaying to Muon Pairs in $2.3 \mathrm{fb}^{-1}$, CDF note 9289 (2008).

[86] D. Beecher, I. Bizjak, C. Hays, B. Jayatilaka, A. Kotwal, T. Riddick, O. StelzerChilton, D. Waters and Y. Zeng, Measurement of the $W$ Boson Mass using 2.2 $\mathrm{fb}^{-1}$ of CDFII Data, CDF note 10755 (2012).

[87] C. Hays, A. Kotwal, L. Nodulman, Curvature Corrections from $E / p$ of Electrons and Positrons in $W \rightarrow e \nu$ Decays, CDF Note 1054 (2011).

[88] R. Fisher, Biometrika 10:507-521 (1915); R. Fisher, Metron 1:3-32 (1921).

[89] Y. Zeng and A. Kotwal, Correlations of $W$ boson mass fits from $m_{T}, p_{T}^{l}$ and $p_{T}^{\nu}$ distributions, CDF note 10373 (2011).

[90] G. Strang, Introduction to Linear Algebra, 3rd edition, Wellesley-Cambridge Press (2003).

[91] L. Lyons, D. Gibaut and P. Clifford, Nucl. Instrum. Methods A 270 (1988).

[92] R.L. Plackett, Some Theorems in Least Squares, Biometrika 37:149-157 (1950). 
[93] D. Beecher, I. Bizjak, C. Hays, B. Jayatilaka, A. Kotwal, T. Riddick, O. StelzerChilton, D. Waters and Y. Zeng, Measurement of the $W$ Boson Mass using 2.2 $\mathrm{fb}^{-1}$ of CDF-II Data, CDF Note 10755 (2012).

[94] W. Press, S. Teukolsky, W. Vetterling and B. Flannery, Numerical Recipes in $C++$, Second Edition, Cambridge University Press (2002).

[95] Private communication with Peter Renton.

[96] The Tevatron Electroweak Working Group, 2012 Update of the Combination of CDF and DØ Results for the Mass of the $W$ Boson, FERMILAB-TM-2532-E, arXiv:1204.0042v2 (2012) 


\section{Biography}

Yu Zeng was born in Jingshan, Hubei, China on July 2nd, 1981. He obtained his B.S. degree from the Department of Physics, Huazhong Normal University in 2002. In 2005, he was awarded The Director's Fellowship and obtained his M.S. degree from Institute of High Energy Physics, Chinese Academy of Sciences in Beijing. He came to study at Duke Physics in August 2005 and worked towards a Ph.D. degree in Physics under the supervision of Prof. Ashutosh Kotwal. During his PhD study, he obtained a M.S. degree in Statistics from the Department of Statistics, Duke University in May 2010. Yu received the Universities Research Association / Fermilab Visiting Scholar Award in March 2010, March 2011 and September 2011. This dissertation was defended on June 18, 2012 at Duke University. 